\title{
Analyzing the eukaryotic translation initiation apparatus and new approaches in affinity chromatography
}

\author{
Dissertation \\ for the award of the degree \\ "Doctor rerum naturalium" (Dr. rer. nat.) \\ of the Georg August Universität Göttingen
}

submitted by

Jennifer Seefeldt

born in

Ellwangen, Germany

Göttingen 2014 
Members of the Thesis Committee:

Prof. Dr. Dirk Görlich, Reviewer

Department of Cellular Logistics

Max Planck Institute for Biophysical Chemistry

Prof. Dr. Marina Rodnina, Reviewer

Department of Physical Biochemistry

Max Planck Institute for Biophysical Chemistry

Prof. Dr. Volker Lipka

Department of Plant Cell Biology

Georg August University Göttingen

Additional Members of the Extended Thesis Committee:

Prof. Dr. Detlef Doenecke

Department of Biochemistry I

Georg August University Göttingen

Prof. Dr. Henning Urlaub

Bioanalytical Mass Spectrometry Group

Max Planck Institute for Biophysical Chemistry

Prof. Dr. Wolfgang Wintermeyer

Department of Physical Biochemistry

Max Planck Institute for Biophysical Chemistry

Date of Thesis submission: 25. September 2014

Date of the oral examination: 14. November 2014 


\section{Affidavit}

I hereby declare that this dissertation "Analyzing the eukaryotic translation initiation apparatus and new approaches in affinity chromatography" has been written independently with no other aids or sources than quoted.

This dissertation has not been submitted elsewhere for any academic award or qualification.

Jennifer Seefeldt

September, 2014

Göttingen, Germany 
To those, who are missed

There's no one in town I know

You gave us some place to go.

I never said thank you for that.

I thought I might get one more chance.

What would you think of me now, so lucky, so strong, so proud?

I never said thank you for that, now I'll never have a chance.

May angels lead you in.

Hear you me my friends.

On sleepless roads the sleepless go.

May angels lead you in

- Jimmy Eat World - 
I am among those who think that science has great beauty. A scientist in his laboratory is not only a technician: he is also a child placed before natural phenomena which impress him like a fairy tale.

- Marie Curie - 


\section{Contents}

Contents vi

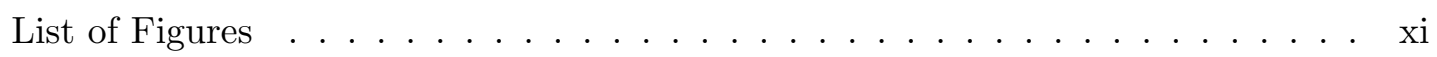

List of Tables . . . . . . . . . . . . . . . . . . . xiii

Abstract xiv

1 Introduction $\quad 1$

1.1 Eukaryotic translation initiation $\ldots \ldots \ldots \ldots . \ldots \ldots$

1.1.1 Eukaryotic translation initiation factor 3 (eIF3) . . . . . . . . . 5

1.2 Spatial separation of transcription and translation is highly beneficial . . . 9

1.2.1 An active transport mechanisms constantly removes translation factors from the nuclear interior . . . . . . . . . . . . 10

1.3 In vitro translation systems enable a closer look into translation processes . 12

1.4 Affinity chromatography . . . . . . . . . . . . . . . . . . . 14

1.4.1 Chemistry for bioactive immobilization of highly selective capture proteins . . . . . . . . . . . . . . . 15

1.4.2 Established affinity tag systems . . . . . . . . . . . . . . 18

1.4.3 RNase Barnase and its inhibitor Barstar . . . . . . . . . . . . 20

1.5 Aim of this study . . . . . . . . . . . . . . . . . . . . 22

2 Results $\quad 23$

2.1 Characterization of recombinant and native wheat eIF3 complex . . . . . 23

2.1.1 All eIF3 subunits can be expressed and purified in a recombinant manner . . . . . . . . . . . . . . . . . 24

2.1 .2 In vitro reconstitution of recombinant eIF $3 \ldots \ldots$. . . . . . . 27

2.1.3 Characterization of native eIF3 in the Wheat Germ Extract . . . . 28

2.1.3.1 Purification and characterization of antibodies against eIF3 28

2.1.3.2 eIF3 forms a stable complex in the wheat germ extract . . 30

2.2 Use of in vitro translation systems to reveal limitations of and necessities for individual translation factors . . . . . . . . . . . . . . . . 33 
2.2.1 Addition of recombinant initiation factors does not stimulate translation efficiency . . . . . . . . . . . . . . . . . . 34

2.2.2 Depletion of eIF3 from wheat germ extracts leads to a reduced translation efficiency . . . . . . . . . . . . . . . . 35

2.2.3 Eukaryotic translation initiation factor 5A (eIF5A) and its effect on poly-proline encoding mRNAs . . . . . . . . . . . . . . 40

2.3 Can nuclear transport factors act as compartment specific inhibitors of translation? . . . . . . . . . . . . . . . . . . . . 43

2.3.1 Addition of Exportin 4 to an in vitro translation system can reduce the translation efficiency of a poly-proline reporter . . . . . . . . . 44

2.3.2 Addition of Crm1 to an in vitro translation system greatly reduces its translation efficiency . . . . . . . . . . . . . . 46

2.3.2.1 Decrease in translation can not be rescued by blocking or mutating the hydrophobic cleft of Crm1 . . . . . . . . . 48

2.4 A activated resin as novel tool for affinity chromatography . . . . . . . . . . 51

2.4.1 MADA Matrix is highly specific for reduced cysteins . . . . . . . . . 52

2.4.1.1 Evaluation of coupling under various conditions . . . . . 53

2.4.2 MADA matrix capacity and background . . . . . . . . . . . . 54

2.4.3 MADA matrix can be used for a variety of applications . . . . . . 56

2.4.3.1 ZZ-Affibody MADA matrix shows less background as commercial IgG matrix . . . . . . . . . . . . . . . 56

2.4.3.2 ProteinA MADA matrix can be used for IP experiments . 58

2.4.3.3 Large complexes can be pulled out by MADA matrix . . . 59

2.4.3.4 MADA - Streptavidin matrix . . . . . . . . . . . . 61

2.5 Barnase:Barstar as novel affinity tag system . . . . . . . . . . . . 63

2.5.1 Identification of Barstar mutants as binding partner for enzymatically inactive Barnase versions . . . . . . . . . . . . . . 64

2.5.2 Structure determination of Barnase:Barstar complex . . . . . . . . 66

2.5.3 Protein Evolution on Barstar . . . . . . . . . . . . . . . . . 68

2.5.3.1 Specific mutations increase Barstar solubility without interfering with Barnase binding . . . . . . . . . . . 69

2.5.3.2 Phage Display . . . . . . . . . . . . . . . . 71

2.5.4 Barnase:Barstar complex from different species are advantageous compared to the B. amyloliquefaciens complex . . . . . . . . 71

2.5.4.1 Barstar from different species show higher solubility than B.amyloliquefaciens Barstar . . . . . . . . . . 71

2.5.4.2 Geobacillus thermoglucosidasius Barnase . . . . . . . 73 
2.5.4.3 Geobacillus thermoglucosidasius complexes are highly stable 74

2.5.4.4 Structure determination of new Barnase:Barstar complexes 76

2.5.5 Evolved Barnase:Barstar complex can be used as tag system for affinity chromatography . . . . . . . . . . . . . . . . 77

3 Discussion $\quad 79$

3.1 Analyzing the eukaryotic translation initiation apparatus . . . . . . . . 79

3.1.1 In vitro reconstitution of the wheat eIF3 complex . . . . . . . . . . 80

3.1.2 In vitro wheat germ translation assay as tool to study effects of translation (initiation) factors . . . . . . . . . . . . . 83

3.2 Nuclear transport factors might act as compartment-specific inhibitors of translation . . . . . . . . . . . . . . . . . . . . . 85

3.2.1 Depletion or sequestration of eIF5A has only subtle effects on the translation of poly proline containing reporters . . . . . . . 86

3.2.2 Addition of Crm1 to an in vitro translation assay decreases translation efficiency dramatically . . . . . . . . . . . . . . 887

3.2.3 Is nuclear translation possible, if NTRs interfere with translation? . 90

3.3 Novel tools for protein purification: a MADA resin and Barnase:Barstar as high affinity pair . . . . . . . . . . . . . . . . 91

3.3.1 MADA activated resins as tailor-made supports for a broad range of applications . . . . . . . . . . . . . . . . 91

3.3.2 Barnase:Barstar affinity tag system . . . . . . . . . . . . . 93

3.3.2.1 Potential application . . . . . . . . . . . . 96

3.4 Perspectives . . . . . . . . . . . . . . . . . . . . . 96

4 Materials and Methods $\quad 98$

4.1 Materials . . . . . . . . . . . . . . . . . . . . . . 98

4.1.1 Chemicals and Reagents . . . . . . . . . . . . . . . . . . . 98

4.1 .2 Instruments . . . . . . . . . . . . . . . . . . . . . . . 98

$4.1 .3 \quad$ Software . . . . . . . . . . . . . . . . . . . . 99

4.1.4 Bioinformatic Resources . . . . . . . . . . . . . . . . . . . . 99

4.1.5 Escherichia coli medium . . . . . . . . . . . . . . . . 100

4.1.6 Escherichia coli strains . . . . . . . . . . . . . . . . 100

4.1 .7 Standard Buffers . . . . . . . . . . . . . . . . . . . . . 101

4.1 .8 Oligonucleotides . . . . . . . . . . . . . . . . . . . . 102

4.1 .9 Gene Synthesis . . . . . . . . . . . . . . . . . . 102

4.1 .10 Antibodies . . . . . . . . . . . . . . . . . . 103

4.1 .11 Plasmids . . . . . . . . . . . . . . . . . . . . . . 104 
4.2 Methods . . . . . . . . . . . . . . . . . . . . . 120

4.2.1 Standard Techniques in Molecular Biology . . . . . . . . . . . . 120

4.2.1.1 Polymerase Chain Reaction (PCR) . . . . . . . . . . . 120

4.2.1.2 Mutagenesis Polymerase Chain Reaction . . . . . . . . . . 121

4.2.1.3 Error-prone Polymerase Chain Reaction . . . . . . . . . . . 121

4.2.1.4 DNA Gel Electrophoresis . . . . . . . . . . . . . . . . 121

4.2.1.5 Purification of DNA Fragments from Agarose Gel and Concentration Determination . . . . . . . . . . . 122

4.2.1.6 Enzymatic Restriction Digest . . . . . . . . . . . . . 122

4.2.1.7 Gibson Assembly Cloning . . . . . . . . . . . . . . . . . . 122

4.2.1.8 Ligation of DNA . . . . . . . . . . . . . . . . 123

4.2.1.9 Transformation of E. coli with DNA by electroporation . . 123

4.2.1.10 Validation of positive clones by testexpression . . . . . . 123

4.2.1.11 Plasmid DNA Purification from E. coli . . . . . . . . . . . 124

4.2.1.12 Primer Synthesis and DNA Sequencing . . . . . . . . . 124

4.2.2 Protein Expression and Purification . . . . . . . . . . . . . . . 124

4.2.2.1 Protein Expression . . . . . . . . . . . . . . . . . . . 124

4.2.2.2 Cell lysis . . . . . . . . . . . . . . . . . . . 125

4.2.2.3 Native Protein Purification . . . . . . . . . . . . . . 125

4.2.2.4 Protease Cleavage . . . . . . . . . . . . . . . 126

4.2.2.5 Buffer Exchange . . . . . . . . . . . . . . . . . . 127

4.2.2.6 Stochiometric complex assembly using two affinity tag systems and an orthogonal protease system . . . . . . . . 127

4.2.2.7 Biotinylation of proteins . . . . . . . . . . . 129

4.2.2.8 Size Exclusion Chromatograph (SEC) . . . . . . . . . . 129

4.2 .2 .9 SDS PAGE . . . . . . . . . . . . . . . 130

4.2.2.10 Western Blot . . . . . . . . . . . . . . . . 130

4.2 .3 Binding Assays . . . . . . . . . . . . . . . . . . . . . . . . . . . 131

4.2.4 Protein Characterization . . . . . . . . . . . . . . . . . 132

4.2.4.1 Protein Identification by Mass Spectrometry . . . . . . . . 132

4.2.4.2 Thermofluor . . . . . . . . . . . . . . . . . . . 132

4.2.4.3 Protein Crystallization . . . . . . . . . . . . . 133

4.2.5 Antibody Purification and Immunoprecipitation Experiments . . . . 134

4.2.5.1 Antibody Production . . . . . . . . . . . . . . . . . . . 134

4.2.5.2 Antibody Purification . . . . . . . . . . . . . . . . . . 134

4.2.5.3 Covalent Coupling of Antibodies to ProteinA . . . . . . . . 135

4.2.5.4 Immunoprecipitation Experiments . . . . . . . . . . . 136 
4.2 .6 In vitro Translation . . . . . . . . . . . . . . . . . . . . 136

4.2.6.1 mRNA preparation . . . . . . . . . . 136

4.2.6.2 In vitro Wheat Germ Translation System . . . . . . . . . . 137

4.2.6.3 In vitro Rabbit Reticulocyte Lysate (RRL) Translation System . . . . . . . . . . . . . . . . 138

4.2.7 Matrix Characterization . . . . . . . . . . . . . . . . . 139

4.2.7.1 Direct coupling . . . . . . . . . . . . . . . . 139

4.2 .8 Indirect pulldowns . . . . . . . . . . . . . . . . . . . . . 140

4.2 .9 Phage Display . . . . . . . . . . . . . . . . . . . . . . 140

4.2.9.1 Library construction . . . . . . . . . . . . . . . . 141

4.2.9.2 Bacteriophage harvest and purification . . . . . . . . 141

4.2.9.3 Phage selection . . . . . . . . . . . . . . . . . . . . 142

4.2.9.4 Analysis of Binders . . . . . . . . . . . . . . . 143

Bibliography

Abbreviations

155

Acknowledgements 157

Curriculum Vitae 


\section{List of Figures}

1.1 Eukaryotic translation initiation . . . . . . . . . . . . . 3

1.2 Two distinct models on subunit composition of eIF3 $\ldots \ldots \ldots$

1.3 3D structure of the human eIF3 complex . . . . . . . . . . . . . . 7

1.4 Facilitated transport between nucleoplasm and cytoplasm . . . . . . . . 11

1.5 Protein purification by affinity chromatography . . . . . . . . . . . 15

1.6 Coupling chemistry to immobilize ligands to a solid phase . . . . . . . 16

1.7 Binding interface of the Barnase:Barstar complex . . . . . . . . . . . . . . 21

2.1 Solubility of eIF3 subunits greatly depend on N-terminal fusion tags . . . 25

2.2 Purification of eIF3 subunits by protease elution . . . . . . . . . . 26

2.3 Purification of antibodies against eIF3 subunits . . . . . . . . . . . . . 29

$2.4 \quad$ Native eIF3 forms a stable complex in the WGE . . . . . . . . . . . 31

2.5 Identification of the native wheat eIF3 complex . . . . . . . . . . . . 32

2.6 Depletion of translation initiation factors decreases overall translation efficiency . . . . . . . . . . . . . . . . . . . 36

2.7 Translation efficiency can be partially restored by adding back recombinant factors . . . . . . . . . . . . . . . . . . . . . 38

2.8 Reporter constructs . . . . . . . . . . . . . . . . . . . . . . 41

2.9 eIF5A depletion does not affect translation of a poly-proline reporter . . . 42

2.10 In vitro translation reporter constructs with N-terminal 3xFLAG tag . . . 44

2.11 Addition of Exportin 4 decreases translation efficiency of proline containing reporters in a Ran dependent manner . . . . . . . . . . . . . . 45

2.12 Nuclear export factor Crm1 decreases overall translation rates in an in vitro translation assay in a Ran dependent manner . . . . . . . . . . . . . 47

2.13 Decrease in translation upon Crm1 addition can not be reversed by blocking or mutating Crm1s hydrophobic cleft . . . . . . . . . . . . . . . . . 49

2.14 Coupling chemistry using maleimide and MADA . . . . . . . . . . 51

2.15 MADA matrix is highly specific towards reduced cysteins . . . . . . . . 52

2.16 Evaluation of coupling under various conditions . . . . . . . . . . . 53

2.17 MADA matrix capacity and background . . . . . . . . . . . 55

2.18 Comparing commercial IgG Sepharose and ZZ-affibody MADA Sepharose 57 
2.19 IP experiments with MADA ProteinA Sepharose . . . . . . . . . . 59

2.20 Large complexes can be pulled out by MADA magnetic beads . . . . . . . 60

2.21 Streptavidin MADA Sepharose efficiently pulls out biotinylated bait from

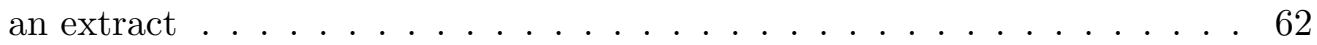

2.22 Barnase $_{\mathrm{H} 102 \mathrm{D}}$ and Barstar $_{\mathrm{C} 42 \mathrm{~K}}$ as ideal affinity pair . . . . . . . . . 65

2.23 Barnase $_{\mathrm{H} 102 \mathrm{D}}:$ Barstar $_{\mathrm{C} 42 \mathrm{~K}}$ complex is extremely salt tolerant . . . . . . . 66

2.24 Purifying the Barnase ${ }_{\mathrm{H} 102 \mathrm{D}}:$ Barstar $_{\mathrm{C} 42 \mathrm{~K}}$ complex . . . . . . . . . . 67

2.25 Crystal structure of the Barnase ${ }_{\mathrm{H} 102 \mathrm{D}}:$ Barstar $_{\mathrm{C} 42 \mathrm{~K}}$ complex . . . . . . . 68

2.26 Mutations on Barstar $_{\mathrm{C} 42 \mathrm{~K}}$ to increase solubility . . . . . . . . . . . . 69

2.27 Barstar sequence alignment . . . . . . . . . . . . . . . . . 72

2.28 Barstars from various species show increased solubility . . . . . . . . . 73

2.29 G.thermoglucosidasius Barnase versions . . . . . . . . . . . . . . . 74

2.30 G.thermoglucosidasius Barstar strongly binds to B.amyloliquefaciens Barnase . . . . . . . . . . . . . . . . . . . . 75

2.31 G.thermoglucosidasius Barnase:Barstar complexes are extremely stable . . 76

2.32 Barnase:Barstar system can be used for affinity chromatography . . . . . 77

4.1 Purification of stochiometric heterodimeric complexes using two affinity tag systems and on-column cleavage . . . . . . . . . . . . . . 128

4.2 In vitro wheat germ translation assay . . . . . . . . . . . . . . . . 137

4.3 In vitro translation using rabbit reticulocyte lysate . . . . . . . . . . . . 138 


\section{List of Tables}

1.1 Cell-free protein expression systems . . . . . . . . . . . . . . . 13

2.1 eIF3 subunit solubility greatly depends on N-terminal fusion tag . . . . 24

2.2 Mutations in Barstar slightly increase solubility . . . . . . . . . . . . . . . 70

$4.1 \quad$ E.coli strains used in this study . . . . . . . . . . . . . . . . 100

4.2 Antibodies used in this study . . . . . . . . . . . . . . . 103

4.3 In vitro translation plasmids used in this study . . . . . . . . . . . . . 104

$4.4 \quad$ E.coli expression vectors used in this study . . . . . . . . . . . . . . 105

4.5 X-ray data collection and refinement statistics Barnase:Barstar complex . 133 


\section{Abstract}

Eukaryotic translation is a multistep process that utilizes 47 individual translation factor subunits. eIF3 is the largest and most complex translation factor, acting during initiation where it promotes assembly of the $43 \mathrm{~S}$ preinitiation complex and the recruitment of the mRNA to the ribosome. Here we analyzed the native wheat eIF3 complex, showing that this 13 subunit factor forms a stable complex in vivo. We were able to recombinantly express and purify the individual subunits under native conditions. However they failed to spontaneously assemble into the holocomplex, suggesting that the maturation of the eIF3 complex requires factors and mechanisms not available when produced in prokaryotes.

Another important aspect of eukaryotic gene expression is the spatial-temporal separation of transcription in the nucleus and translation in the cytoplasm. Cells evolved mechanisms to keep the levels of translation factors in the nuclear interior continually low. Exportins actively transport these proteins to the cytoplasm in a RanGTPase-driven manner. Crm1 is known to interact with almost all translation factors; Exportin 4 specifically removes eIF5A from the nucleus. We could show that addition of Crm1 and Exportin 4 to an in vitro translation system decreases translation rates of reporter constructs in a RanGTP dependent manner, supporting the assumption that nuclear transport factors act as compartment specific inhibitors of translation.

In addition, we present a new tool for affinity chromatography based on a MADA activated solid phase and a novel affinity tag system. The solid phase reacts specifically with thiol groups, allowing to immobilize any ligand that contains an exposed and reduced cysteine. Affinity resins based on the MADA chemistry compare very favorable to commercially available systems, in particular in terms of reduced background binding under low salt conditions.

The streptavidin:biotin system is routinely used as state of the art technique for affinity purifications. Nonetheless it has severe disadvantages, namely the tetrameric state of streptavidin, poor folding of streptavidin as well as biotin contamination during bacterial expression/purification and bait proteins require covalent modification with biotin. We now developed an alternative based on the extremely tight B.amyloliquefaciens Barnase:Barstar complex $\left(\mathrm{K}_{\mathrm{D}}=10^{-14} \mathrm{M}\right)$. To allow for separate cytoplasmic expression of these modules, we detoxified the RNase Barnase by a His ${ }_{102}$ Asp mutation and restored high binding strength by a compensatory $\mathrm{Cys}_{42}$ Lys mutation on Barnase inhibitor Barstar. The $1.98 \AA$ crystal structure indeed shows that the mutated residues form a shielded salt bridge that further stabilizes the complex. Barstar initially showed poor solubility upon overexpression in E.coli. This problem was solved by switching to the Barnase:Barstar pair identified in the hyperthermophilic Bacillus relative Geobacillus thermoglucosidasius. 


\section{Introduction}

The "Central Dogma of Molecular Biology" presents the flow of genetic information in a biological system. It was first stated by Francis Crick in 1958 and re-stated in 1970 (Crick, 1958, 1970). Although by now - 56 years later - more about the multitude of possibilities of how information is processed in a cell is known, the dogma still guides scientists through the main steps in gene expression.

The genetic information of all living organisms is encoded in their DNA. When required, parts of the information is transferred to messenger RNA (mRNA) in a process called transcription. mRNA molecules are decoded to an amino acid sequence during a process termed translation. The emerging polypeptide folds to a three dimensional structure, thereby resulting in a protein that is now able to function in the cell. Although these major steps are common in bacteria and in eukaryotes, they differ dramatically in terms of complexity and factors involved.

In comparison to bacteria, eukaryotes evolved compartmentalized cells thereby restricting certain processes to membrane enclosed organelles. The cell nucleus is the cells most prominent organelle, first spotted in 1719 by Antonie van Leeuwenhoek (Delphis et al., 1719). When looking at gene expression, transcription and translation in bacteria act in a coupled manner and regulation mechanisms mainly affect transcription. In eukaryotes however, a spatial-temporal separation of transcription and translation can be observed. Transcription happens exclusively in the nuclear interior. The produced mRNA is further processed and subsequently transported through nuclear pores to the cytoplasms where finally translation occurs. Thus, gene expression can be regulated at additional steps in eukaryotes, being it the processing and transport or the translation itself. That eukaryotic translation is certainly more complex and thus likely to be more regulated as in bacteria can already be seen when comparing the amount of proteins involved in translation. Only nine different polypeptides are required for proper bacterial translation whereas 47 are necessary in eukaryotes.

In general, translation can be divided into four major steps: initiation, elongation, termination and ribosome recycling. During translation initiation, an elongation-competent $80 \mathrm{~S}$ ribosome is assembled on the start codon of the mRNA. Thereby, the correct start codon is recognized by the initiator tRNA (Met-tRNA $\left.{ }_{i}{ }^{M e t}\right)$ when located in the P-site of 
the ribosome.

Translation elongation generally describes the peptide bond formation between subsequent amino acids forming the polypeptide chain. After initiation, the start codon is located in the ribosomes P-site, base-paired with the anticodon of the initiator tRNA. The second codon of the open reading frame $(\mathrm{ORF})$ is located in the A-site. Cognate aminoacyl-tRNAs are guided to the A-site by elongation factor eEF1A, a GTPase that upon base-pairing hydrolyzed GTP. Thereby the tRNA is released and accommodates into the A-site. Peptide bond formation between the first methionine and the new amino acid occurs, catalyzed by the peptidyl transferase center (PTC) of the ribosome. Simultaneously, the peptidyltRNA translocates from the A-site to the P-site, catalyzed by elongation factor eEF2, yet another GTPase. A new cognate aminoacyl-tRNA can now bind to the free A-site and the cycle reiterates (reviewed in Dever and Green, 2012).

Translation termination occurs as soon as a stop codon locates to the A-site of the ribosome and is facilitated by two proteins that form a 1:1 complex: the eukaryotic release factor 1 (eRF1) and the GTPase eukaryotic release factor 2 (eRF2). eRF1 recognizes the stop codon and thereby triggers GTP hydrolysis on eRF2, promoting peptide bond cleavage and the release of the nascent polypeptide chain (Frolova et al., 1996; Alkalaeva et al., 2006). The last step of translation is the recycling of the ribosomal subunits. After nascent chain release, the ribosomal subunits, the mRNA, the deacetylated tRNA in the $\mathrm{P}$-site and the release factors remain attached to each other, forming the post-translation complex (post-TC complex). This complex can be dissociated either by the translation initiation factors eIF3, eIF1 and eIF1A (Pisarev et al., 2007), or by the ATP-binding cassette (ABC) protein ABCE1 (Pisarev et al., 2010). Re-association of the ribosomal subunits is prevented by eIF1, eIF1A and eIF3 remaining bound to the $40 \mathrm{~S}$ ribosomal subunit. In addition, eIF6 binds the 60S ribosomal subunit at its ribosomal subunit interface (Russell and Spremulli, 1979).

\subsection{Eukaryotic translation initiation}

As mentioned earlier, eukaryotic translation initiation requires 47 different proteins to function properly. Interestingly, the number of factors needed in each of the four steps of translation is not evenly distributed. Most of the factors - 37 in total - are needed during translation initiation. Thus, initiation seems to be the most regulated and rate limiting step during the fundamental process of protein synthesis.

In general, initiation describes the recruitment of the mRNA to the small ribosomal subunit, subsequent scanning of the complex for the first AUG codon and base-pairing of the initiator tRNA (Met-tRNA ${ }_{i}{ }^{\text {Met}}$ ) with the start codon and finally ribosomal subunit joining resulting in an elongation-competent $80 \mathrm{~S}$ ribosome placed on the mRNAs ORF. 
The detailed function and action of all initiation factors are described in the following. A schematic representation of eukaryotic translation (initiation) can be seen in figure 1.1.

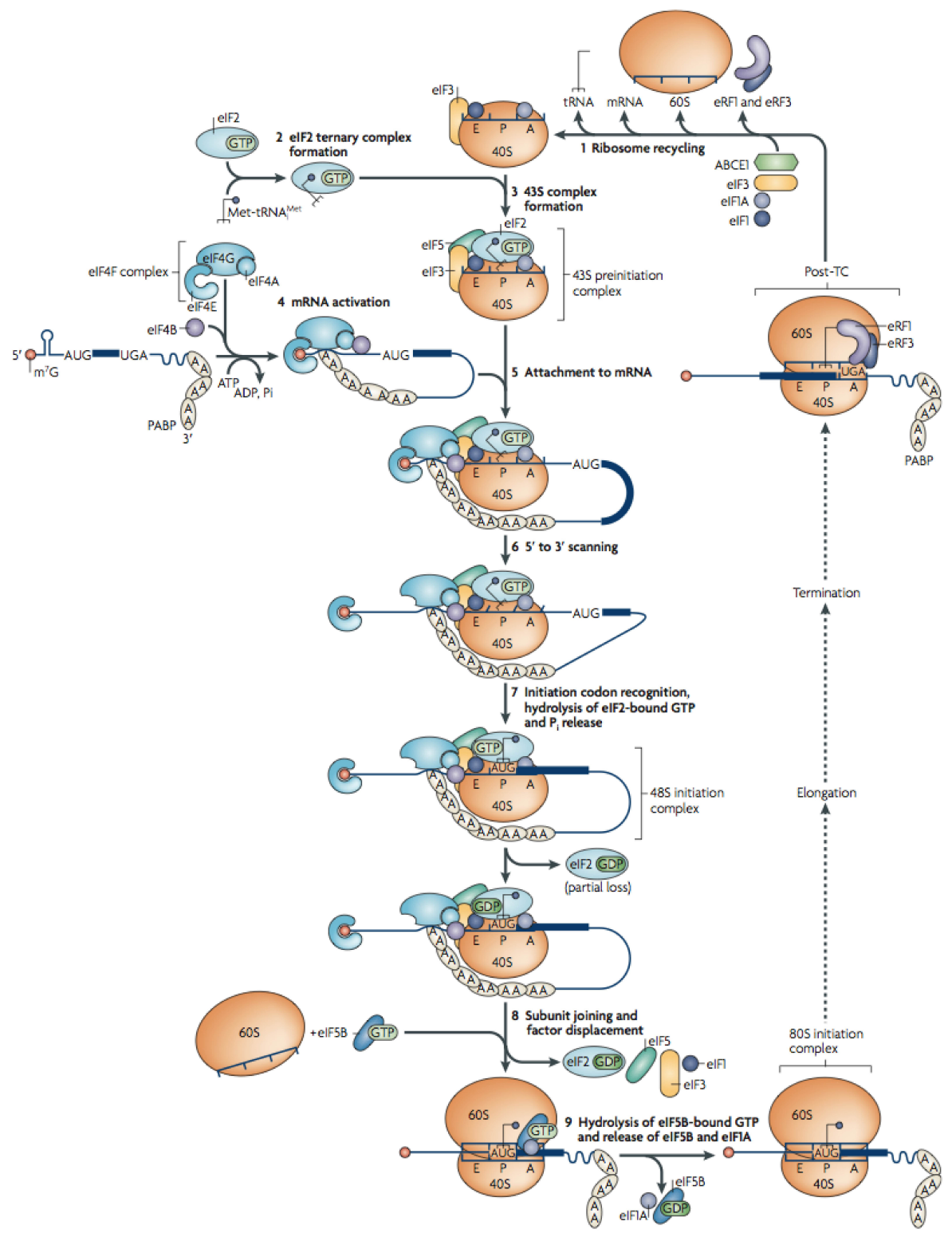

Figure 1.1: Eukaryotic translation initiation

Eukaryotic translation initiation factor 2 (eIF2) in complex with GTP binds the initiator tRNA (Met-tRNA $\mathrm{A}_{\mathrm{i}}{ }^{\mathrm{Met}}$ ) thereby forming the ternary complex (TC). The TC associates with the ribosomal 40S subunit, which is already bound by eIF3, eIF1 and eIF1A. By additional binding of eIF5, the 43S pre-initiation complex (PIC) is formed. In parallel, the 7-methylguanylate $\left(\mathrm{m}^{7} \mathrm{G}\right)$ cap at the $5^{\prime}$ end of the mRNA is recognized by the cap-binding complex eIF4F, which contains eIF4E, eIF4G and the ATP-dependent helicase eIF4A (acts together with eIF4B). eIF4G also interacts with the poly-A binding protein (PABP), thereby circularizing the mRNA transcript. By binding of the 43S PIC and the complexed mRNA, the $48 \mathrm{~S}$ pre-initiation complex is formed. The complex now scans the mRNA for the start AUG (must be located in the P-site of the ribosome), which is recognized by base-pairing with the anticodon of the Met-tRNA ${ }_{i}{ }^{\text {Met }}$. Upon pairing, GTP in eIF2 is hydrolyzed and eIF2-GDP, eIF3, eIF5 and $\mathrm{EIF} 1$ are released, allowing eIF5B-GTP and the $60 \mathrm{~S}$ ribosomal subunit to bind. Upon proper subunit joining, GTP is hydrolyzed and eIF5B-GDP and eIF1A are released. The fully assembled 80S ribosome is now able to translate the ORF of the transcript (elongation). When a stop codon is located in the ribosomes A-site, translation termination takes place, performed by the release factors eRF1 and eRF2. The post-termination complex (Post-TC) is disassembled by ABCE1, eIF3, eIF1A and eIF1 in a process termed ribosome recycling. (adopted from Jackson et al., 2010) 
Translation is a cyclical process, meaning that ribosomes involved in the translation of an ORF are recycled and can participate in another round of protein synthesis. During ribosome recycling, the ribosomal subunits are dissociated either by the ATP-binding cassette (ABC) protein ABCE1 (Pisarev et al., 2010) or by the translation initiation factors eIF1, eIF1A and eIF3 (Pisarev et al., 2007). The mode of dissociation depends on the concentration of nucleotide-unbound $\mathrm{Mg}^{2+}$. After dissociation, eIF1, eIF1A and eIF3 either remain bound to the $40 \mathrm{~S}$ ribosomal subunit or they rapidly bind when dissociation was performed by ABCE1 to prevent re-association of the two ribosomal subunits.

In parallel, the eIF2 complex (in its GTP bound state) binds the initiator tRNA (Met$\mathrm{tRNA}_{\mathrm{i}}{ }^{\mathrm{Met}}$ ), thereby forming the ternary complex (TC), which is subsequently attached to the 40S ribosomal subunit by interactions between eIF2 - eIF3 and eIF2 - eIF1A (Valasek et al., 2002; Olsen et al., 2003). The resulting 43S pre-initiation complex now comprises the $40 \mathrm{~S}$ ribosomal subunit, initiation factors eIF1, eIF1A, eIF2-GTP-Met-tRNA ${ }_{i}{ }^{\text {Met }}$ and additionally bound eIF5, a GTPase-activating protein (GAP; Saini et al., 2014). eIF2 is built up from three different subunits: $\operatorname{eIF} 2 \alpha, \operatorname{eIF} 2 \beta$ and $\operatorname{eIF} \gamma$. eIF $2 \alpha$ is thought to be the regulatory subunit of the complex, as its serine residue $S_{51}$ can be phosphorylated upon distinct types of stress (e.g. amino acid deprivation, ER stress or heme deficiency) leading to sequestration of the guanine nucleotide-exchange factor (GEF) eIF2B (Kimball et al., 1998; Donnelly et al., 2013). Therefore, no new ternary complexes can be formed as they require eIF2 in a GTP bound state, leading to an overall decrease of translation. eIF2 $\beta$ and eIF2 $\gamma$ both bind the guanine nucleotide and Met-tRNA ${ }_{i}^{\text {Met }}$ (Gaspar et al., 1994).

Eukaryotic mRNAs are modified such that their 5' end contains a 7-methylguanylate $\left(\mathrm{m}^{7} \mathrm{G}\right)$ cap and their 3' end harbors a poly-adenosine (polyA) tail. The tail is recognized by the Poly(A)-binding protein PABP, the 5' cap is recognized by the eIF4F complex comprising the direct binding protein eIF4E, the scaffolding protein eIF4G and a DEADbox RNA helicase eIF4A (Pestova et al., 1996). eIF4As activity is enhanced by either eIF4B or eIF4H (Rogers et al., 2001). Together they are required to unwind the secondary structures of the 5' untranslated regions (UTRs) of the mRNA to allow scanning of the ribosome. The mRNA is recruited to the 43S pre-initiation complex by the direct binding between eIF4G and the eIF3e subunit (LeFebvre et al., 2006).

Next, the ribosome scans the mRNAs 5'UTR. The scanning-competent conformation of the small ribosomal subunit is thereby induced by eIF1 and eIF1A (Passmore et al., 2007). A lack of eIF1 or eIF1A lead to strongly reduced or no scanning ability. Scanning complexes must be able to discriminate between the real AUG start codon and codons that partially base-pair with the initiator tRNA. Thus, not only the AUG is required to sit in the $\mathrm{P}$-site of the ribosome, in addition, the AUG codon needs an optimal context. Ideally a purine is positioned in the -3 position and a guanine in the +4 position (terming the 
A of the start codon as +1 position; Kozak, 1991). The most important initiation factor enhancing the initiation fidelity is eIF1. When codon anticodon base-pairing between the start AUG and the initiator tRNA is optimal, a tightening of the eIF1A - 40S interaction occurs, thereby displacing eIF1 from the P-site. This triggers a conformational change in the small ribosomal subunit, thereby locking it tightly to the mRNA (Maag et al., 2005). Initiation codon recognition also triggers the activity of the GTPase activating protein eIF5 that is bound to eIF $2 \beta$ but activates the GTPase activity of eIF $2 \gamma$. GTP is hydrolyzed, thereby reducing the affinity of the eIF2 complex towards the tRNA that can now occupy the P-site (Kapp and Lorsch, 2004). eIF2-GDP partially dissociates from the complex. The following $60 \mathrm{~S}$ subunit joining and the displacement of eIF1, eIF1A, eIF3 and eIF2 is mediated by the ribosome-dependent GTPase eIF5B (Pestova et al., 2000) resulting in an elongation-competent $80 \mathrm{~S}$ ribosome placed on the mRNA.

In comparison to the vertebrate translation apparatus, the same process in plants shows some additional characteristics most likely evolved to match the requirements evoked by unique cellular process, e.g. photosynthesis or plant hormone signaling. Plants show two types of the cap-binding complex eIF4F. The eIF4isoF shares the eIF4A helicase with the eIF4F complex, but the other two subunits, eIF4isoE and eIF4isoG are different. It has been suggested that the two eIF4F variants can discriminate between cap-dependent initiation and translation starting at internal ribosome entry site (IRES) (Gallie and Browning, 2001). In addition, plants have a noval cap binding protein (nCBP) that was first identified in A.thaliana, however its detailed function and need is so far unclear (Ruud et al., 1998).

\subsubsection{Eukaryotic translation initiation factor 3 (elF3)}

Eukaryotic translation initiation factor 3 (eIF3) is the largest and most complex initiation factor first identified and purified from rabbit reticulocyte in 1976 (Benne and Hershey, 1976). eIF3 is involved in most reactions occurring in the initiation pathway, thereby organizing a web of interactions between several translation initiation factors. In vertebrates and plants, the eIF3 complex consists of 13 nonidentical subunits, termed eIF3a-eIF3m and making up a mass of $\sim 800 \mathrm{kDa}$ (Asano et al., 1997; Browning et al., 2001). In budding yeast, the eIF3 complex is only made up by 5 subunits, orthologous to the mammalian subunits eIF3a, eIF3b, eIF3c, eIF3g and eIF3i, indicating a conserved core complex. In addition, budding yeast contains a protein orthologous to eIF3j, which is substochiometric and nonessential (Phan et al., 1998). However, not only the five eIF3 core subunits seem to be required for proper eIF3 function. Experiments in fission yeast showed that although eIF3f and eIF3m are not part of the conserved core complex, they are absolutely essential for viability (Akiyoshi et al., 2001). 
The first eIF3 subunit interaction studies were performed in vivo using budding yeast. By genomic deletion of predicted binding domains in tagged eIF3 subunits and subsequent determination of co-purified sub complexes, first models on yeast eIF3 subunit compositions and their interactions towards other initiation factors were reported (Phan et al., 1998; Valasek et al., 2002). Similar interactions were also shown in mammals including additional information on the interactions between core and non-core subunits, e.g. between eIF3b and eIF3e (Shalev et al., 2001).
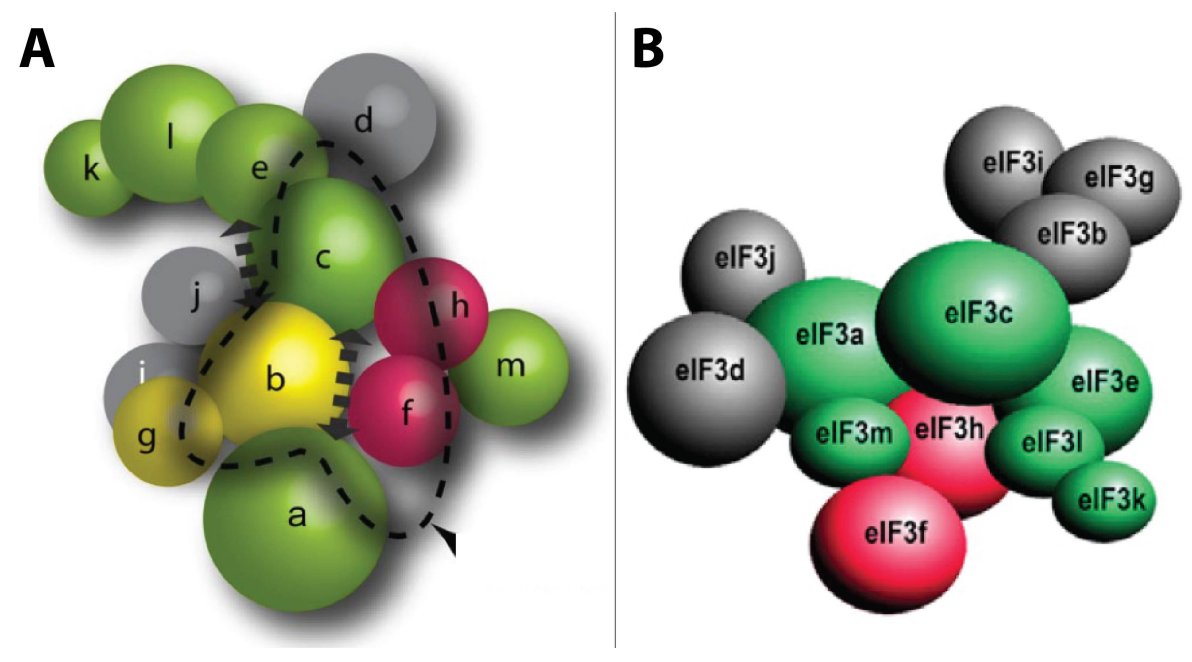

Figure 1.2: Two distinct models on subunit composition of elF3

Recently, two different subunit compositions for the humane eIF3 complex have been reported. (A) Zhou et al. (2008) used a mass spectrometry approach to probe the subunit interactions within the complex. By "solution disruption experiments", different subcomplexes were determined resulting in the shown model. The color code represents subunits containing PCI-domains (green), MPN-domains (red) or RNA-recognition motifs (yellow). (B) shows the subunit composition experimentally validated by Sun et al. (2011) and rephrased by Querol-Audi et al. (2013). Sun and colleagues reconstituted the 13-subunit eIF3 complex in E.coli by using a stepwise assembly of coexpressed subcomplexes thereby mapping the interactions between the individual subunits. PCI-domain containing subunits are depicted in green, MPN-domain containing subunits are shown in red.

(adapted from Zhou et al., 2008; Querol-Audi et al., 2013)

Nowadays, two models on human eIF3 subunit composition are available as shown in figure 1.2. Zhou et al. (2008) analyzed a natively purified 13-subunit eIF3 complex by tandem mass spectrometry. By performing "solution disruption experiments", they were able to detect three stable modules (eIF3(c-d-e-l-k), eIF3(f-h-m), eIF3(a-b-i-g)), which are brought together by interactions between subunits eIF3b and eIF3c and eIF3c and eIF3h. Their model was further confirmed by immunoprecipitation experiments. The second composition is shown in figure $1.2 \mathrm{~B}$, based on a stepwise reconstitution of the human eIF3 complex in E.coli (Sun et al., 2011). The authors claim a stable 8-mer core comprised of the PCI/MPN subunits eIF3a, eIF3c, eIF3e, eIF3f, eIF3h, eIF3k, eIF3l and eIF3m.

The first 3D structure of human eIF3 was determined using complexes natively purified from HeLa cell lysate. At a resolution of $30 \AA$, a body-like shape for eIF3 was determined, showing a head domain, a left and right arm and left and right leg domain (Siridechadilok et al., 2005). Recent data at higher resolution reveal a more detailed view on the human 
eIF3 complex, however, the five extended domain shape remains as shown in figure 1.3A (Querol-Audi et al., 2013). Cryo electron microscopy was performed with reconstituted human eIF3 complexes, using the protocol published by Sun et al. (2011). This strategy enabled to add tags on certain subunits, thereby being able to determine the localization of individual subunits in the 3D model. eIF3h (marked in red) seems to be positioned in the center of the complex, which fits to the mass spectrometry data suggesting eIF3h to play a role in linking individual subcomplexes. Figure 1.3B shows the obtained eIF3 structure modeled onto the 40S ribosomal subunit. The location of the complex on $40 \mathrm{~S}$ was previously suggested by Siridechadilok et al. (2005). The 3D model also contains the initiation factors eIF1 (blue) and eIF3 (yellow) that play a major role in correct AUG recognition during scanning of the mRNA by the $43 \mathrm{~S}$ PIC.
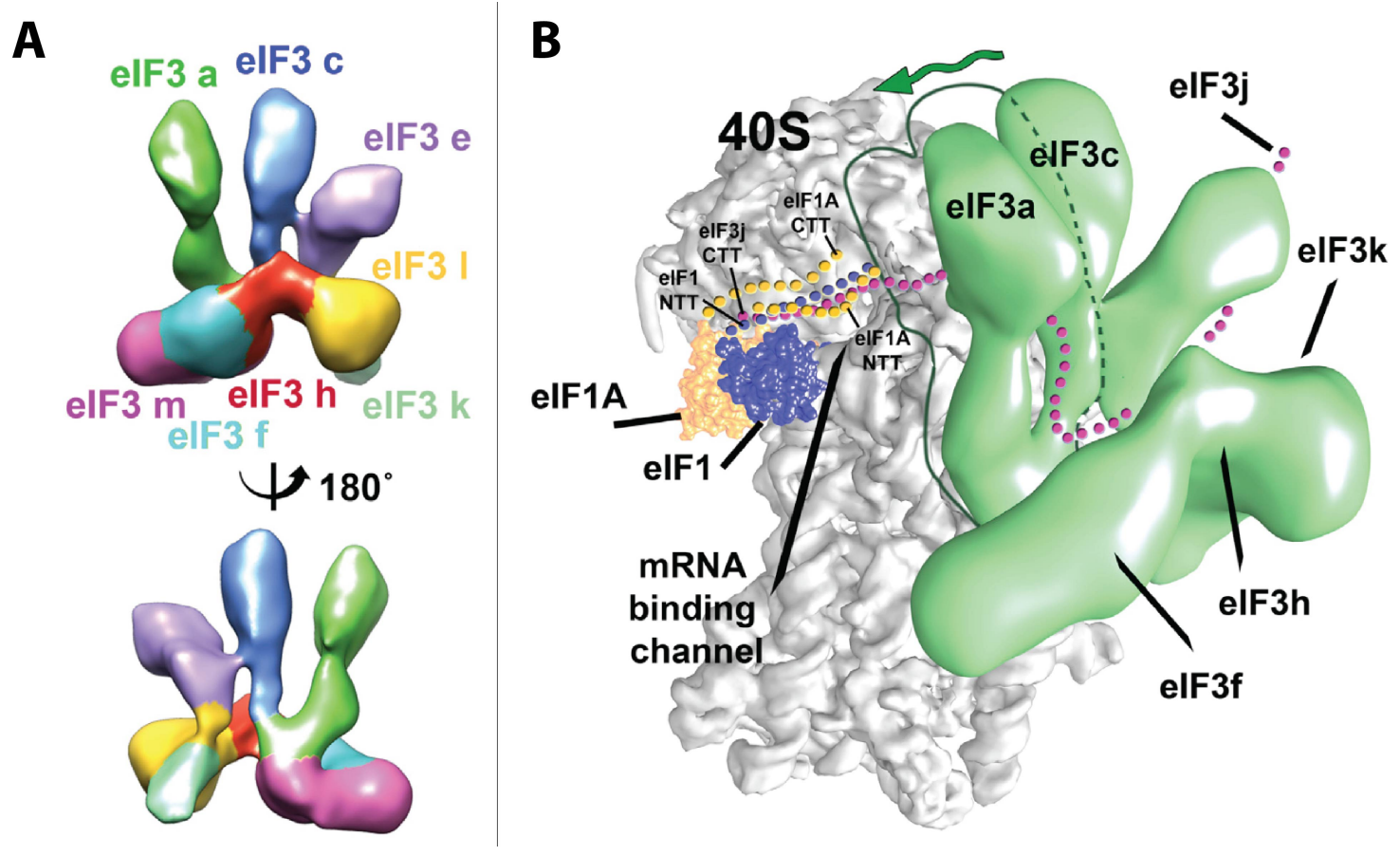

Figure 1.3: 3D structure of the human elF3 complex

(A) By utilizing genetic tag visualization by electron microscopy at a resolution of $12 \AA-16 \AA$, the location of the octameric eIF3 core subunits in the 13-subunit human eIF3 complex could be revealed (Querol-Audi et al., 2013). (B) The 3D reconstitution of the eIF3 complex was modeled onto the 40S ribosomal subunit together with initiation factors eIF1(blue) and eIF1A (yellow). eIF3 was placed according to experimental data by Siridechadilok et al. (2005), eIF1 and eIF1A due to crystal data (Rabl et al., 2011). Potential localization of eIF3j is marked by the magenta dots.

(adopted from Querol-Audi et al., 2013)

As indicated earlier, eIF3 functions in various steps during translation initiation. These steps can be categorized as follows: (1) eIF3 acts as assembly platform for 43S PIC formation. In yeast, eIF3 forms a multifactor complex (MFC) together with eIF1, eIF1A, eIF2 and eIF5. Thereby, eIF3 is the central factor in this complex holding it together. The MFC then binds the 40S ribosomal subunit in a cooperative manner (Asano et al., 2000). In mammals eIF3 stimulates binding of the ternary complex to the 40S ribosomal subunit, 
which is strongly impaired when eIF3b is mutated (Phan et al., 1998). In vitro studies further showed that the eIF3a-eIF3b-eIF3c subcomplex by itself can bind the 40S ribosomal subunit and recruits the ternary complex (Phan et al., 2001). (2) eIF3 is essential for mRNA recruitment to the $43 \mathrm{~S}$ PIC. Generally, eIF3 interacts with eIF4G, member of the cap binding eIF4F complex, thereby tethering the mRNA to the small ribosomal subunit (Korneeva et al., 2000). However, mRNA recruitment can also happen in an eIF4G independent manner. In yeast, some mRNAs can be directly recruited by the eIF3 complex; in mammals, some mRNAs are attached by an interaction between eIF3 and eIF4E bound to the $\mathrm{m}^{7}$ GTP cap (Jivotovskaya et al., 2006). (3) eIF3 is required for mRNA scanning and AUG initiation fidelity. eIF3c interacts with eIF5, the GTPase activating protein, and eIF1. When mutating eIF3c, binding of the other two translation initiation factors is impaired and a dramatic increase of initiation events at non-AUG (e.g. UUG) codons can be observed (Asano et al., 2000). The chain of interactions between eIF3, eIF1 and eIF5 seems to be crucial for proper accommodation of the initiator tRNA to the ribosomal P-site. When eIF5 triggers GTPase activity on eIF2, eIF3 seems to slightly shifted thereby displacing eIF1, which is known to bind very close to the P-site. (4) eIF3 can induce reinitiation events and thereby plays a major role in gene-specific translational control. In yeast eIF3 is required for reinitiating events when translating the GNC4 mRNA, in plants eIF3 is miss-used for repeating reinitiating events during the translation of a viral, polycistronic mRNA (Park et al., 2001). (5) eIF3 prevents rejoining of 40S and 60S. Although the bulk mass of the eIF3 complex is though to bind to the solvent side of the 40S ribosomal subunit, an extended domain seems to bind to the interface surface thereby preventing and disrupting intersubunit binding (Siridechadilok et al., 2005). Thereby the eIF3 subunits eIF3d and eIF3j are though to play a major role in vertebrates, whereas subunits eIF3a and eIF3c are required for 40S binding in yeast (Fraser et al., 2004; Nielsen et al., 2006).

Although much seems to be known about the function and interactions of and within the eIF3 complex, many details remain unclear. So far, mainly human and budding yeast eIF3 complexes were analyzed; the plant complex however remains greatly unstudied. It is known that eIF3 in plants also contain 13 subunits, however the arrangement of the subunits in the complex and their interaction within the complex are unknown and there might be further eIF3 subunits or isoforms so far not identified.

Recent studies showed that the human eIF3 complex can be reconstituted using either the Bacoluvirus system (Masutani et al., 2007) or even E.coli (Sun et al., 2011). These major breakthroughs enable a new level of analyzing this large factor by introducing mutations or deletions that would lead to a lethal phenotype in in vivo experiments. Recombinant 
reconstitution further allows to create high yields of pure complex that might even be modified such that flexible regions are deleted and thus could potentially crystalize. In addition, the striking difference in subunit amount between the eIF3 complexes from budding yeast and vertebrates/plants raise the question which of the higher eukaryotic subunits are truly required for eIF3 function and which subunits are needed for the complex regulatory network eIF3 is involved in.

\subsection{Spatial separation of transcription and translation is highly beneficial}

In eukaryotes, the two main processes during gene expression are separated in a spatialtemporal manner. Transcription occurs in the nuclear interior, translation happens in the cytoplasm. This separation is highly beneficial for several reasons. Most eukaryotic mRNAs show an alternating structure of exon and intron regions. During a process termed splicing, which is exclusively nuclear, the intron regions are cleaved out resulting in fused exons that make up the open reading frame of the protein to be produced. Intron regions often harbor pre-mature stop codons as these regions do not (or only slightly) underlie evolutionary pressure against them. If translation happens in the nuclear interior, the probability of translating non-spliced or incompletely spliced mRNA greatly increases. The resulting truncated proteins can have severe dominant negative effects, thereby reducing cell viability. In addition, proteins translated in the nucleus might negatively affect gene expression as they are mislocalized and usually function in other compartments.

Despite all these negative impacts, ideas about translation taking place also in the cell nucleus persist since first reported in 1954 (Allfrey, 1954). The author claimed a rapid incorporation of radioactive amino acids into nuclear proteins. The debate was further encouraged by Goidl et al. (1975), reporting the isolation of polyribosomes from nuclei. Nowadays, the hypothesis of nuclear translation is based on three findings: tRNAs are synthesized, maturated and aminoacylated within nuclei (Lund and Dahlberg, 1998), an observed coupled process of transcription and translation in isolated nuclei of mammalian cells (Iborra et al., 2001) and the presence of rRNA and protein components of the translation machinery at sites of active transcription in Drosophila cell nuclei (Brogna et al., 2002). However, many of these findings were at least partially disproven or explained differently by other laboratories. Nathanson et al. (2003) were able to show that the ability of isolated nuclei to perform translation reduces in proportion to the purity of the nuclei sample, concluding that the observed translation events are performed by ribosomes still attached to the co-isolated endoplasmatic reticulum or other cytoplasmic contaminations. Concerning the translation machinery components present in the nuclear interior, it must 
be clearly distinguished between presence and function. Ribosomes, respectively, are partially assembled in the nuclear interior, however, the final steps of maturation occur only in the cytoplasm. In addition, ribosome assembly factors remain associated to the $60 \mathrm{~S}$ ribosomal subunit during maturation, preventing 40S association (Udem and Warner, 1973). Further, Bohnsack et al. (2002) showed that levels of most translation factors present in the nucleus are kept low. GFP-tagged translation factors were actively exported to the cytoplasm by the nuclear transport machinery.

\subsubsection{An active transport mechanisms constantly removes translation factors from the nuclear interior}

Although the process of translation is restricted to the cytoplasm, some translation factors can be detected in the nuclear interior at low concentrations. The factors are either small enough to freely diffuse through nuclear pore complexes, or they harbor amino acid sequences that are misinterpreted as nuclear localization signal. In most eukaryotic cells, an intermixing between nucleoplasm and cytoplasm occurs during nuclear envelope breakdown. Mislocalized proteins need to be actively shuttled back to the cytoplasm.

The nucleus is a membrane-enclosed organelle. Molecules produced in the interior such as RNAs or ribosomal subunits need to be transported through the membrane (nuclear envelope) to the cytoplasm whereas proteins translated in the cytoplasm but acting in the nucleus need to be imported back (Bonner, 1975). Hence, trafficking between nucleus and cytoplasm is absolutely essential. The main route of transport between the two compartments are nuclear pore complexes, gigantic protein assemblies (125MDa in vertebrates; Reichelt et al., 1990) punctuating the nuclear envelope. Proteins with a diameter $<5 \mathrm{~nm}$ can freely diffuse through the pore (Mohr et al., 2009), whereas larger proteins or complexes require nuclear transport receptors (NTRs) for facilitated transport.

Nuclear transport receptors are large molecules with sizes of $90-150 \mathrm{kDa}$, all part of the importin $\beta(\operatorname{Imp} \beta)$ family. They can be classified according to the direction of transport in nuclear export factors (exportins) and nuclear import factors (importins) (Görlich et al., 1994; Fornerod et al., 1997). The directionality of transport requires the small GTPase Ran. It can switch between an active RanGTP state that is able to bind to NTRs and a non-active state after GTP hydrolysis (RanGDP). RanGTP concentrations in the nucleoplasm are significantly higher than in the cytoplasm, resulting in a steep gradient across the nuclear envelope (Görlich et al., 1996).

Importins bind their cargo at low RanGTP levels in the cytoplasm. Most import cargos harbor classical nuclear localization signals (NLS) that are recognized directly by the importin. Alternatively, cargos are bound by an adaptor, e.g. Importin $\alpha$ that interacts with Importin $\beta$ via its IBB domain (Görlich et al., 1995). After translocation through the 


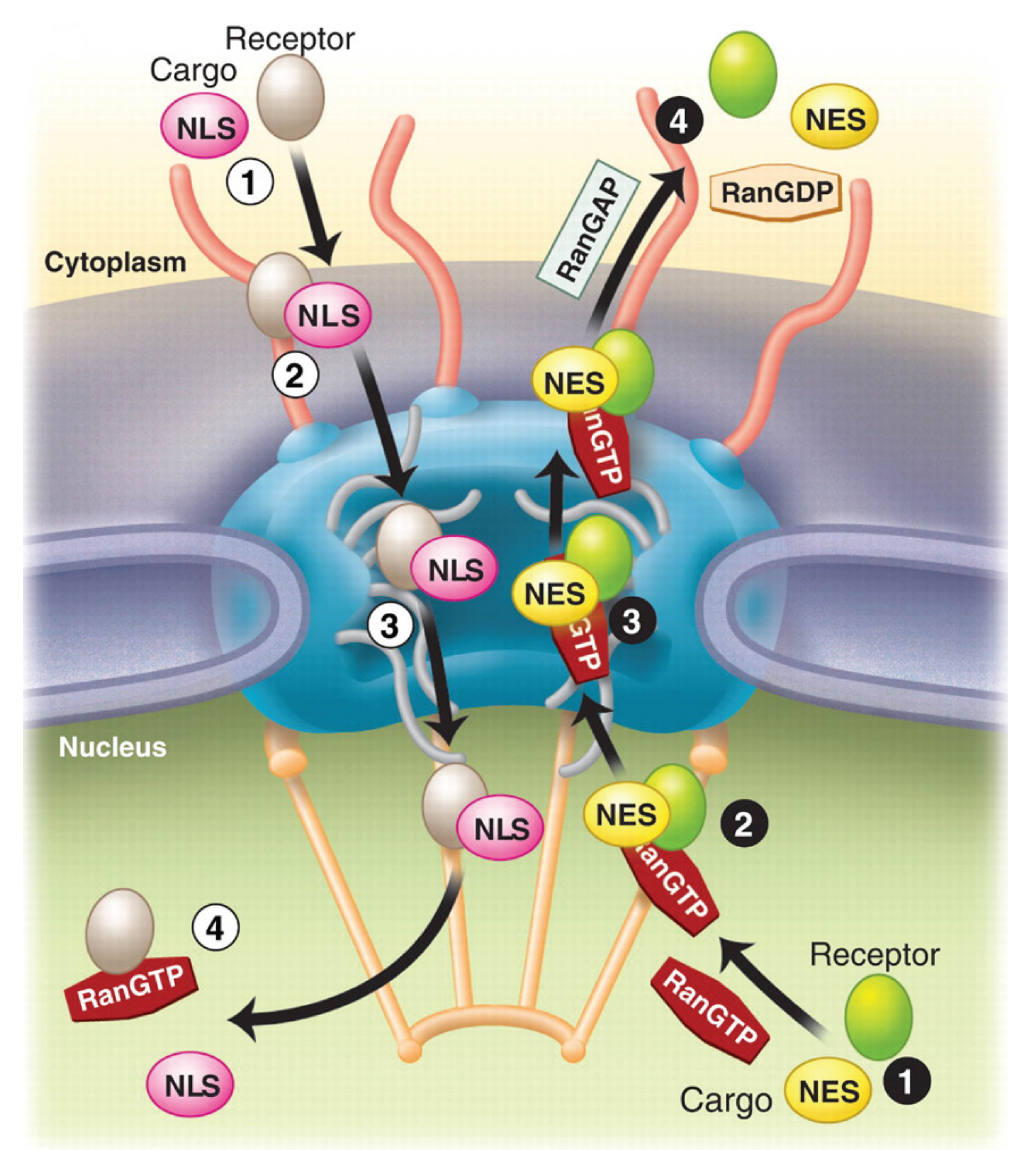

Figure 1.4: Facilitated transport between nucleoplasm and cytoplasm

Facilitated transport is mediated by nuclear import receptors (importins) or nuclear export receptors (exportins). The directionality of transport is thereby generated by a RanGTP gradient between the two compartments: RanGTP concentrations in the nucleus are high, whereas RanGTP concentrations in the cytoplasm are low (Görlich et al., 1996). During cargo import (white numbering), the import receptor binds its cargo either directly via the nuclear localization signals (NLS) or by adaptor proteins such as Importin $\alpha$. This complex translocates to the nuclear interior by interacting with FG-domains of nucleoporins that revet the central channel of the pore. In the nucleoplasm, the receptor-cargo complex disassembles by binding of RanGTP. During protein export (black numbering) the export receptor binds its cargo in a RanGTP dependent manner. After shuttling through the nuclear pore, RanGAP activates Ran GTPase activity, GTP is hydrolyzed and the receptor-cargo complex disassembles to RanGDP, the cargo and the export receptor. NES (nuclear export signal) stretches are recognized by the export factor Crm1, other exportins recognize their cargo by other sequence characteristics.

(adapted from Terry et al., 2007)

nuclear envelope, RanGTP binds the import receptor, thereby disassembling the receptorcargo complex and releasing the cargo. Importins bound to RanGTP shuttle back to the cytoplasm, where GTP is hydrolyzed and RanGDP is subsequently released (figure 1.4, white labeling). During nuclear export, exportins bind their cargo in the nuclear interior in a RanGTP dependent manner. The RanGTP-receptor-cargo complex passes the nuclear envelope and RanGTP is hydrolyzed to RanGDP in the cytoplasm, triggered by RanGAP. The complex disassembles, the cargo is released and the export receptor can shuttle back to the nucleoplasm (reviewed in Görlich and Kutay (1999); figure 1.4, black labeling). Re-entering of Ran to the nucleus is mediated by the specialized transport receptor NTF2 (Ribbeck et al., 1998). 
Experiments performed by Bohnsack et al. (2002) showed that many translation initiation and elongation factors are actively removed from the nuclear interior by nuclear export factors. eEF1A in complex with tRNA and RanGTP can be shuttled to the cytoplasm by Exportin 5, Exportin 4 mediates export of the translation factor eIF5A (Lipowsky et al., 2000) and Importin13, a NTR that can transport cargos in both directions, brings eIF1A back to the cytoplasm (Mingot et al., 2001). It was additionally shown that export of eIF $2 \beta$ from the nucleus is sensitive to leptomycinB, a drug that inhibits the nuclear export factor Crm1 (Nishi et al., 1994; Bohnsack et al., 2002). Recent unpublished data show that almost all translation initiation factors can be pulled out from HeLa cell lysate by Crm1 in a RanGTP dependent manner (K. Kirli, unpublished).

We hypothesize that nuclear transport factors capable of binding translation factors can act as compartment specific inhibitors of translation in the nucleus, thereby providing yet another strong argument against the hypothesis of protein synthesis occurring in the nuclear interior.

\subsection{In vitro translation systems enable a closer look into translation processes}

In vitro translation systems are a widespread tool in the fields of molecular biology and biochemistry. It can be either used to produce proteins for further downstream applications or to study the translation mechanisms and the regulatory network involved.

The first cell-free translation system was obtained from unfractionated E.coli extract, containing all components for transcription and translation allowing protein synthesis from a DNA template (Nirenberg and Matthaei, 1961). The first eukaryotic in vitro translation system was based on the rabbit reticulocyte lysate, enabling mRNA dependent protein synthesis (Pelham and Jackson, 1976). The best-studied plant cell-free translation system is obtained from wheat germ, first reported by Roberts and Paterson (1973). Although these three systems are well established nowadays and commercially available, new cell-free translation systems are intermittently reported, based on insect cell lysate (Ezure et al., 2006), the eukaryotic parasite Leishmania tarentolae (Kovtun et al., 2011) or even human HeLa cell lysate (Mikami et al., 2008). However, these systems were so far only tested on a small group of proteins and characterization of posttranslational modifications and proper protein folding of the synthesized polypeptide are still required. Table 1.1 summarizes the main aspects of the cell-free translation systems.

The bacterial in vitro translation system has extremely low production costs and proteins can be produced to high yields. The possibility to work with lysates obtained from genetically modified strains can be highly beneficial, e.g. by deleting regions coding for 
nucleases or proteases. The commercially available recombinantly reconstituted E.coli translation machinery (PURE system) is a fast and efficient way of protein synthesis and allows analysis of the bacterial translation apparatus without the unknown and uncharacterized activities present in a lysate based system (Shimizu et al., 2001). However, the production of eukaryotic proteins in a bacterial system is problematic as these are often produced in an insoluble manner and no posttranslational modifications are introduced.

Table 1.1: Cell-free protein expression systems

Comparison of established bacterial (E.coli) and eukaryotic in vitro translation systems, reviewed in Jackson and Hunt (1983); Madin et al. (2000); Kigawa et al. (2004); Ezure et al. (2006); Mikami et al. (2008); Kovtun et al. (2011). PTM= post translational modifications

\begin{tabular}{|c|c|c|c|c|}
\hline System & Yield & Costs & PTM & Additional Information \\
\hline E.coli & high & low & no & $\begin{array}{c}\text { recombinant system } \\
\text { available (PURE), strains } \\
\text { can be genetically modified }\end{array}$ \\
\hline Leishmania tarentolae & ng-mg range & low & not tested & $\begin{array}{c}\text { new system, limited } \\
\text { number of proteins tested, } \\
\text { more soluble protein in } \\
\text { comparison to E.coli }\end{array}$ \\
\hline Insect cell lysate & low & high & yes & $\begin{array}{l}\text { translation of large } \\
\text { proteins possible, } \\
\text { cap-independent } \\
\text { translation, formation } \\
\text { of disulfide bonds }\end{array}$ \\
\hline Wheat Germ Extract & ng-mg range & moderate & $\begin{array}{c}\text { yes }(\text { no N- } \\
\text { glycosylation) }\end{array}$ & $\begin{array}{l}\text { high solubility rates, } \\
\text { cap-independent } \\
\text { translation possible }\end{array}$ \\
\hline Rabbit Reticulocyte & ng range & high & $\begin{array}{c}\text { yes }(\text { no N- } \\
\text { glycosylation) }\end{array}$ & $\begin{array}{c}\text { Mammalian system, } \\
\text { cap-independent } \\
\text { translation, high } \\
\text { hemoglobin concentrations }\end{array}$ \\
\hline Human & low & high & yes & $\begin{array}{c}\text { newly established } \\
\text { system, limited number } \\
\text { of proteins tested }\end{array}$ \\
\hline
\end{tabular}

To circumvent these problems, large or eukaryotic chaperone-dependent proteins are often produced in an eukaryotic in vitro translation system. The cytoskeleton protein actin, respectively, can not be solubly expressed in a bacterial based translation system but functional, proper folded protein can be obtained from eukaryotic based cell-free translation systems (personal communication). Both, the rabbit reticulocyte lysate (RRL) and the wheat germ extract (WGE) system are established and well studied systems tested for a great variety of proteins. Nevertheless, the WGE has significant advantages over the RRL system. First, the production of RRL is very cost intensive and severe treatment on the 
animals is required. The wheat germ extract is produced from winter wheat seeds, which is available in unlimited amounts and raises less ethical concerns. Second, the RRL main protein is hemoglobin, making up $\sim 90 \%$ of the protein content in the lysate interfering with downstream applications if not removed beforehand. The proteins translated in the wheat germ extract can however be immediately utilized for a broad range of applications and assays without prior treatment. Third, although the yields for both systems are significantly lower as compared to bacterial systems, the wheat germ extract obtains the highest yields among eukaryotic translation systems (ng - mg range).

In this study, eukaryotic in vitro translation systems based on the RRL and the WGE system are used to analyze the translation (initiation) apparatus. The main advantage of a cell-free approach is the ability to massively manipulate the system, introducing loss-offunction effects that would cause immediate lethality when occurring in living organisms.

\subsection{Affinity chromatography}

Affinity chromatography is a powerful and fundamental technique used in biochemistry to separate proteins or protein complexes of interest from crude extracts or other complex mixtures. It is based on the interaction between a protein and its cognate ligand. The first purification of a protein due to the biospecific adsorption onto a solid phase was described in 1910 for $\alpha$-amylose binding to starch (Starkenstein, 1910). The term "affinity chromatography" was coined by Cuatrecasas et al. (1968), reporting the purification of $\alpha$-chymotrypsin by specific binding to its inhibitor carboxypeptidaseA that was coupled to a sepharose based solid matrix. Nowadays the technique evolved such that affinity tag systems enable to purify any protein of interest, thereby not being dependent on a natural interaction between the target protein and a ligand. Further, the technique is not exclusively used for protein purification but also for protein interaction analysis such as immunoprecipitation experiments or pull-down assays.

Protein purification can be performed by a variety of methods, such as selective precipitation, size exclusion or differential centrifugation. However, a highly selective purification of the protein of interest can be achieved best by affinity chromatography, schematically depicted in figure 1.5. The protein of interest is genetically fused to an affinity tag, containing the affinity protein and - if required - a protease cleavage site. This fusion construct is expressed in E.coli respectively, resulting in a cell lysate containing vast amounts of different proteins including the expressed fusion protein. The lysate is added to a stationary phase, a solid material to which the ligand has been covalently attached. Proteins containing the affinity tag specifically bind to the resin whereas other sample components do not show any affinity and are hence washed off. Elution of the protein of interest can be achieved by two procedures shown in figure $1.5 \mathrm{E}$ and $1.5 \mathrm{~F}$, depending on further 

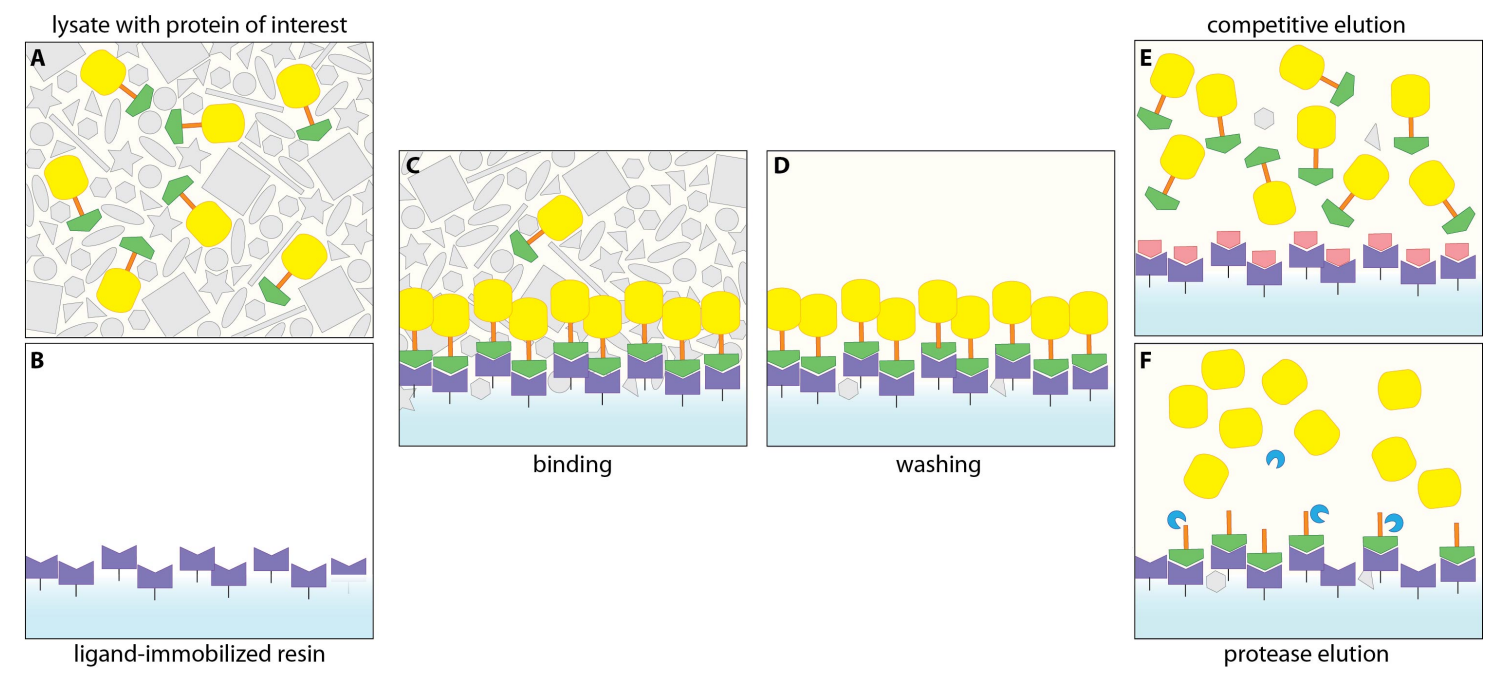

Figure 1.5: Protein purification by affinity chromatography

Affinity chromatography is based on a strong interaction between a protein and its cognate ligand. (A) For protein purification, the protein of interest (yellow) is fused to the affinity tag (green) linked by a protease cleavage site (orange), if required. The fusion protein is expressed in E.coli respectively resulting in a lysate containing a multitude of proteins including the fusion protein. (B) The stationary phase during affinity chromatography is a solid material covalently linked to the affinity pair ligand (violet). (C) The lysate and the resin are mixed, allowing the fusion proteins to bind to the resin whereas other proteins do not show any binding affinity towards the stationary phase. After washing (D), the fusion protein (and some contaminants (grey)) remain bound to the resin. All other proteins are removed. Elution of the protein of interest can be performed either by competition (E) or by protease cleavage (F). During competitive elution, a strong binder (red) displaces the entire fusion protein from the ligand. During protease elution, small amounts of highly active protease (blue) are added to the fusion protein bound resin and during incubate, the protease specifically cleaves at the protease cleavage site of the fusion protein. This results in release of the tag-free protein of interest and the fusion tag (green and orange) still bound to the resin.

downstream applications. By competitive elution, the entire fusion protein is release from the stationary phase. A selective competitor is added in high concentrations, thereby replacing the fusion protein from its ligand. The most prominent example is the release of histidine-tagged proteins from a $\mathrm{Ni}^{2+}$-chelate resin by imidazole. The protein of interest can also be released by protease elution. Thereby specific proteases (depicted in blue in figure 1.5) act on the protease cleavage site of the fusion construct, releasing the tag-free protein of interest from the resin. The tag (affinity protein and remaining protease cleavage site) remains bound to the stationary phase. This elution procedure is highly selective as only proteins containing the protease cleavage site are affected and unspecifically binding proteins remain on the resin. Additionally, elution can also be performed by using denaturing agents or changing parameters such as $\mathrm{pH}$ or ionic strength.

\subsubsection{Chemistry for bioactive immobilization of highly selective capture proteins}

As mentioned previously, affinity chromatography requires a mobile phase, e.g. the lysate containing the fusion protein, and a solid phase, usually a stationary material to which the ligand is covalently attached. The ideal matrix is chemically and mechanically stable, insoluble in the solvent used during the experiment, has low non-specific binding properties and a high surface to volume ratio, and should allow chemical activation for efficient 
attachment of the biospecific ligand. Commonly used resins can be classified as follows: (1) resins based on natural occurring polycarbohydrates such as agarose, dextrane or cellulose; (2) synthetic materials such as polyacrylamide or polystyrene (Staak et al., 1996); (3) inorganic particles such as glass or porous silica (Xi and Wu, 2004); (4) superparamagnetic beads, which are extremely beneficial for accumulation of low abundance proteins in a complex mixture (Safarik and Safarikova, 2004).

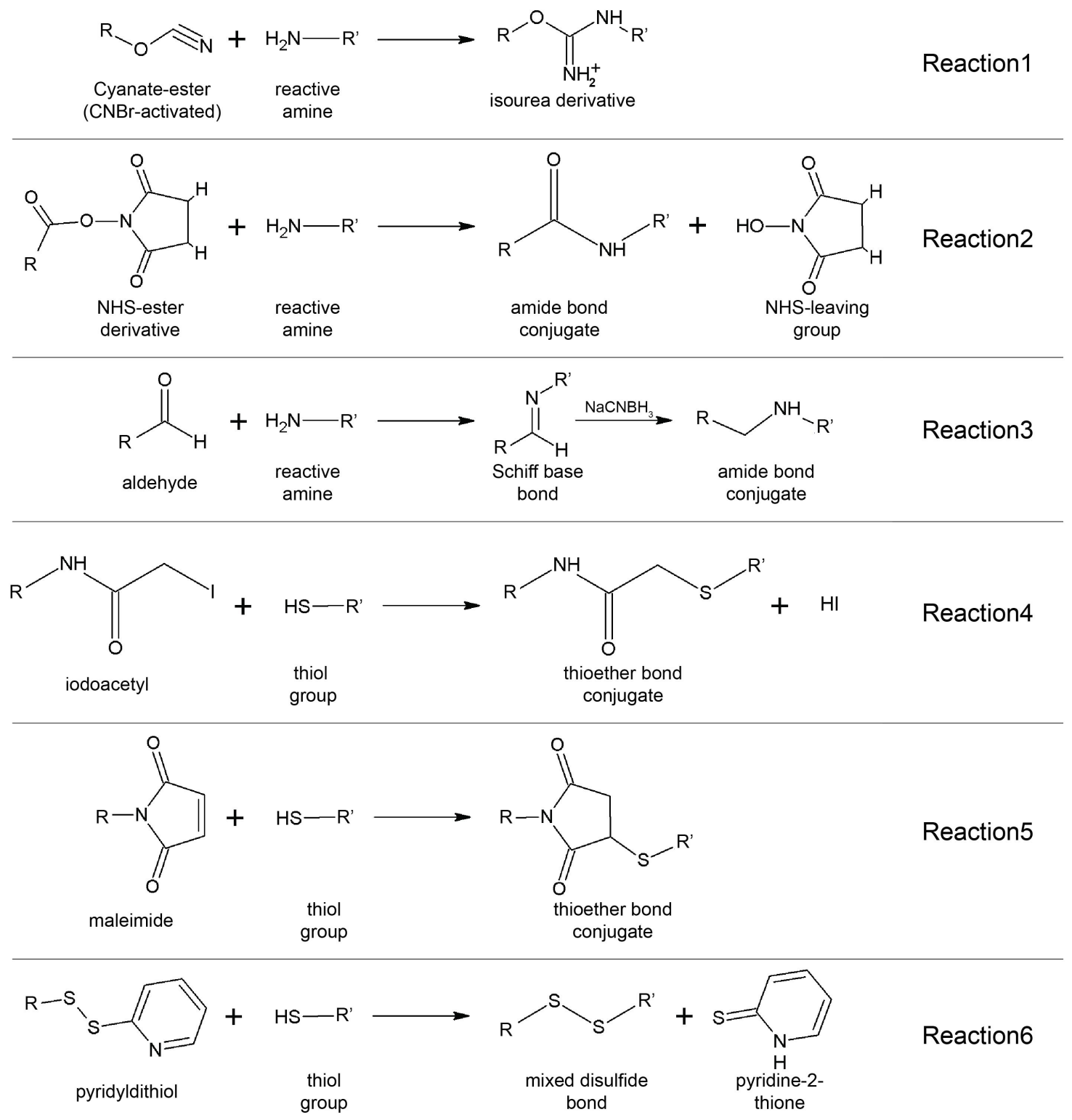

Figure 1.6: Coupling chemistry to immobilize ligands to a solid phase

Immobilization of peptides or proteins to a solid phase require the activation of the phase with compounds reactive towards functional groups on the protein surface, mostly primary amines or thiolgroups. Coupling through amine groups can be performed by CNBR-activated cyanate-esters (Reaction 1), NHS-esters (Reaction 2) or aldehyde groups (Reaction 3), all resulting in an amide bond conjugate. When coupling is performed using a thiol group of the protein, supports are activated with haloacetamide derivatives such as iodoacetyl (Reaction 4), with maleimides (Reaction 5) or with pyridyldithiol (Reaction 6). Iodoacetyl and maleimides result in thioester bond conjugates, the reaction with pyridyldithiol leads to a mixed disulfide bond. 
The covalent attachment of a ligand to a solid phase requires the activation of the phase with a compound that is reactive towards a functional group on the ligand, most common primary amines or sulfhydryl groups (overview shown in figure 1.6). Early immobilization protocols used cyanogen bromide $(\mathrm{CNBr})$ activated supports able to form an isourea linkage with the primary amine group of the protein to be attached (Axén and Ernback, 1971; figure 1.6 Reaction 1). However, this coupling chemistry has severe disadvantages, mainly the formation of a highly instable isourea bond, leading to leakage of the ligand from the solid phase. Ideal coupling chemistries should neither affect the structure or function of the ligand, nor the structure of the resin. Furthermore the produced bond should be absolutely stable to prevent leakage and the activated resin should not promote unspecific binding of proteins due to chemical properties.

The most commonly used functional group for ligand immobilization are primary amines, found at the N-terminus of polypeptide chains that are not acetylated and in lysine side chains. Besides other chemistries, coupling can be performed utilizing aldehyde or NHSester activated solid phases. Aldehyde activated resins react with amino groups, leading to the formation of a Schiff base intermediate that is further reduced by e.g. sodium cyanoborohydride $\left(\mathrm{NaCNBH}_{3}\right)$. Upon reduction, the linkage stabilizes by forming a secondary amine (Peng et al., 1987; figure 1.6 Reaction 3). NHS-esters are formed by first activating carboxylate groups with a carbodiimide, e.g. 1-Ethyl-3-(3-dimethylaminopropyl)carbodiimide (EDC) and second, the condensation of the intermediate with NHS (NHydroxysuccinimide; Williams et al., 1981). This intermediate can be easily displaced by nucleophilic attacks from primary amine groups, resulting in a covalent amide bond between the carboxyl group and the ligand and an NHS leaving group (figure 1.6, Reaction $2)$.

Another frequently used functional group for protein immobilization is the sulfhydryl or the thiol group present in cystein side chains. In contrast to primary amines, thiol groups are more seldom in a polypeptide chain, allowing a direct and orientated coupling. In addition ligands can be modified such, that cysteins are placed at defined sites, allowing a coupling distant from the binding site of the ligand towards its target. Thiol groups tend to form disulfide bonds, thereby stabilizing secondary or tertiary structures of a protein. However, this bonds need to be reduced to sulfhydryl groups again prior to immobilization. Thiol groups are commonly attached to a resin using haloacetamide derivatives such as iodoacetamides. At neutral or slightly alkaline conditions, the halogen group is displaced upon a nucleophilic attack by the thiol group, resulting in the formation of a thioether linkage and release of hydrogen iodide, HI (figure 1.6 Reaction 4).

Maleimide is another reactive compound that can be used to attach cystein containing proteins to a solid phase. Thereby the double bond of the maleimide reacts with the sulfhydryl 
group of the ligand, thereby forming a stable thioether bond (Smyth et al., 1964; figure 1.6 Reaction 5). Interestingly, this chemistry is so far mainly used for fluorescent labeling of proteins or crosslinking but rarely for immobilization. Yet another possibility to covalently couple ligands with cysteins utilizes pyridyl disulfide derivatives. These compounds react with thiol groups thereby forming a new, mixed disulfide bond, that can be cleaved again by reducing agents such as DTT (figure 1.6 Reaction 6). This coupling strategy is not useful for affinity chromatography, as reductants in lysates would cause leakage of the ligand from the resin, however it is frequently used for covalent chromatography.

\subsubsection{Established affinity tag systems}

After discussing the different stationary materials and coupling chemistries that can be used to generate the solid phase for affinity chromatography, we now focus on the affinity pair itself that allows the separation of specific proteins or protein complexes from a complex protein mixture.

All proteins are involved in specific non-covalent interactions with other molecules, termed ligands, that can be either low molecular weight substances or other proteins. Binding can occur via hydrophobic or charged patches on the protein surface or by short-range molecular interactions such as hydrogen bonds or van der Waals forces. These specific interactions are exploited in affinity chromatography systems, attaching one member of the affinity pair to the solid phase and fusing the other member - the so-called affinity tag - to the protein aimed to be purified. In general, one can distinguish small peptide tags, such as FLAG-, poly-histidine-, S-, c-myc-, or StrepII-tag, and the attachment of folded domains or entire proteins such as MBP, GST or the ZZ domain.

The most common affinity tag system is the binding of poly-histidine stretched to chelated metal ions on a resin, based on the immobilized metal affinity chromatography (IMAC) technique reported by Porath et al. (1975). Divalent transition metal ions such as $\mathrm{Co}^{2+}$, $\mathrm{Ni}^{2+}, \mathrm{Cu}^{2+}$ or $\mathrm{Zn}^{2+}$ are immobilized to the solid phase by surface-bound chelators such as NTA (nitrilotriacetic acid; Hochuli et al., 1987). Remaining free valencies are able to form coordinated bonds with the electron donor groups present in amino acid side chains, most efficiently with the electron donor groups of the imidazole ring found in histidine side chains. Under native conditions, $\mathrm{His}_{3}$-tags are efficient for stable binding (Janknecht et al., 1991), however His6- or His $10^{-}$-tags are commonly used as the interaction strength increases with the number of histidines (avidity effect). Elution of the fusion protein occurs by addition of imidazole, which competes with the poly-histidine tag thereby replacing it. This affinity tag system can be used for native or denaturing protein purification, the polyhistidine stretch can be attached to the $\mathrm{C}$ - and $\mathrm{N}$-terminus of a protein and recombinant 
expression was demonstrated in bacteria, yeast and mammals.

Other small peptide affinity tags are:

- the 15 amino acid S-tag that recognized the S-protein. Both fragments derive from RNAseA, which is naturally exposed to limited proteolysis in the cell. After cleavage, the two fragments remain strongly but non-covalently bound to each other (Karpeisky et al., 1994). The S-tag itself is known to have solubility enhancing properties and is therefore frequently used as fusion tag, whereas the S-protein is immobilized to the stationary phase.

- the eight amino acid overall hydrophilic FLAG-tag that is specifically recognized by M1 antibody (Hopp et al., 1988). It is mostly used under non-denaturing conditions and elution is performed by either reducing the $\mathrm{pH}$ or by specific elution using antigenic peptides.

- the 11 amino acid c-myc epitope, termed c-myc-tag, that is recognized by the Mab9E10 antibodies attached to a stationary phase. Elution of the tag is achieved by decreasing the $\mathrm{pH}$ of the buffer or by addition of competitive peptides (Evan et al., 1985).

Note that c-myc and FLAG-tags are rarely used for preparative affinity purification as these systems are extremely cost intensive, but are frequently used for protein detection and characterization techniques due to their high binding specificity and the possibility to elute by specific peptides.

Instead of fusing small affinity peptides to the protein of interest, folded domains or entire proteins can be attached as well. These often have a dual function in acting as affinity tag and as solubility enhancer. However, these fusion tags often need to be removed from the purified protein to prevent interference with downstream applications due to their size or chemical properties. The $42 \mathrm{kDa}$ ProteinA, found in Staphylococcus aureus, is a commonly used affinity tag. It contains five homologous domains, which are all capable of binding to the Fc-domains of immunoglobins (IgGs). One of these domains, the B-domain, was engineered such that it is more tolerant towards site-specific chemical cleavage, resulting in the 7kDa Z-domain (Duhamel et al., 1979; Moks et al., 1986; Nilsson et al., 1987). ZZ-domain or ProteinA fusion proteins can be purified by IgG coupled resins, vice versa ProteinA resins can be used to efficiently purify IgGs from sera. The recently described engineered affibody Zpa963 raised against ProteinA (Lindborg et al., 2013) provides yet another ligand to purify ZZ-tagged proteins. Other frequently used affinity tags are GST (Glutathione S-transferase), maltose binding protein (MBP) or NusA, which requires addition of a His-tag. GST is a $26 \mathrm{kDa}$ protein that efficiently binds to glutathione immobilized on a solid phase and can be displaced again by addition of free glutathione (Smith and 
Johnson, 1988). However, GST is not able to fold when the fusion protein is attached to its N-terminus and it forms homodimers, which might be problematic during the expression of fusion proteins with exposed hydrophobic surfaces. The $40 \mathrm{kDa}$ protein MBP can be purified on cross-linked amylose and subsequently eluted by high concentrations of free maltose (di Guan et al., 1988). It is known to increase the solubility of its fusion partners due to its chaperone-like qualities and thus is often used for the expression of eukaryotic proteins in a bacterial environment (Sachdev and Chirgwin, 1999; Smyth et al., 2003). MBP is often used in combination with small affinity peptides such as His-tags to enable the purification via highly efficient $\mathrm{Ni}^{2+}$-chelate resins and a protease cleavage site to produce the protein of interest in a tag free form. Another protein frequently used as solubility enhancer in combination with a His-tag for affinity purification is the E.coli NusA protein, which is $50 \mathrm{kDa}$ in size and extremely hydrophilic (Davis et al., 1999).

To be able to efficiently remove large solubility enhancing affinity tags, the fusion construct should contain a protease cleavage site. The seven amino acid recognition site for the TEV (Tobacco Etch Virus) protease can be found in a multitude of commercially available vector systems (Dougherty et al., 1988). However, the usage of small ubiquitinlike modifier (SUMO) sequences as system for tag removal shows two major advantages in comparison to TEV. The $10 \mathrm{kDa}$ SUMO protein shows solubility enhancing properties and fusion proteins can be specifically and more efficiently be cleaved off by the SUMO protease when fused to the C-terminus of the SUMO protein (Marblestone et al., 2006; Frey and Görlich, 2014a).

An affinity system that is not only used for protein purification but also for high-throughput screening methods and protein characterization is the streptavidin:biotin system. Streptavidin is produced by Streptomyces avidinii and binds biotin with a remarkable high affinity of $\mathrm{K}_{\mathrm{D}} \sim 10^{-14}$ (Chaiet et al., 1963; Green, 1990). Usually, streptavidin is immobilized to the stationary phase and the biotin moiety is introduced to the protein of interest either chemically or enzymatically. For enzymatic modification, small peptide tags such as the 15 amino acid Avi-tag were evolved that can be fused to the protein of interest and be in vivo or in vitro biotinylated by the enzyme BirA (Schatz, 1993).

To avoid additional modification of the fusion protein, Strep-tags were developed that are able to bind to streptavidin and even more efficient to Strep-Tactin, a streptavidin derivative (Schmidt et al., 1996; Voss and Skerra, 1997).

\subsubsection{RNase Barnase and its inhibitor Barstar}

Although the streptavidin:biotin pair is highly beneficial for affinity chromatography due to their strong interaction, the system has severe disadvantages, mainly due to the homotetrameric nature of streptavidin. Although a single molecule is only $\sim 15 \mathrm{kDa}$ in size, this 
adds up to a large complex when oligomerized and immobilized to a resin. Other natural occurring binding pairs with comparable binding constants could be more beneficial for affinity chromatography applications.

Barnase is a small (110 amino acids) secreted extracellular ribonuclease produced by the bacterium Bacillus amyloliquefaciens. The 89 amino acid Barstar protein, the specific inhibitor for Barnase, is produced by the same organism and acts intracellularly to protect itself (Hartley, 1989). The two molecules interact in a 1:1 stochiometric manner with a $\mathrm{K}_{\mathrm{D}} \sim 10^{-14}$ and an association rate constant of $4 \times 10^{8} \mathrm{M}^{-1} / \mathrm{s}$ (Mariani et al., 1992; Schreiber and Fersht, 1993).

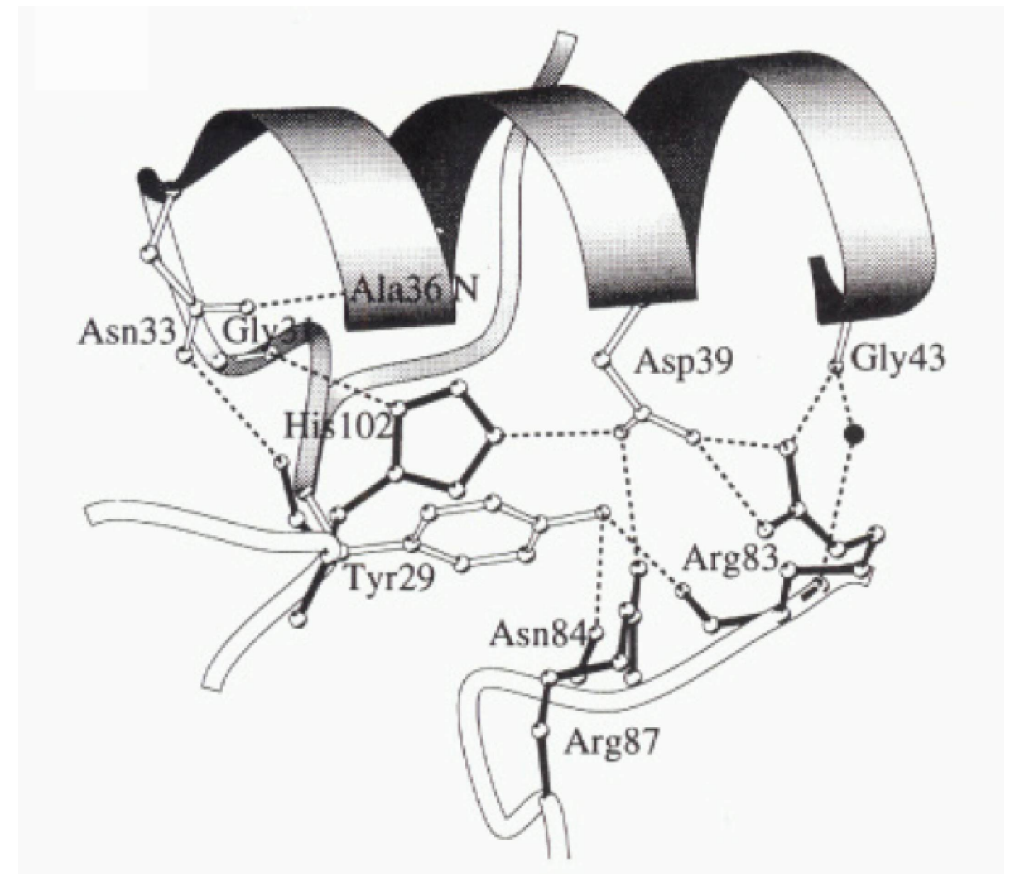

Figure 1.7: Binding interface of the Barnase:Barstar complex

Schematic representation of the binding interface between Barnase (white) and its inhibitor Barstar (gray). Barstars $\mathrm{Asp}_{39}$ on its $\alpha$-helix 2 forms hydrogen bonds with the basic residues $\operatorname{Arg}_{83}, \operatorname{Arg}_{87}$ and $\mathrm{His}_{102}$ in the active site of Barnase, thereby facilitating the strong interaction between the two molecules. Further the Barnase enzymatic activity is inhibited by interacting with the catalytic residue His 102 . Adopted from Buckle et al. (1994)

The X-ray structure of the Barnase:Barstar complex was solved at a resolution of $2.6 \AA$ in 1993 (Guillet et al., 1993), revealing the mode of interaction between the two molecules and the inhibition of the RNase activity. Barnase cleaves RNA molecules using a general acid-base catalysis mechanism, thereby utilizing Glu 73 and $\mathrm{His}_{102}$. The catalytic center is penetrated by $\alpha$-helix $_{2}$ of Barstar and the loop region connecting helix 2 and helix $3 . \mathrm{Mu}-$ tagenesis analysis identified Asp $_{39}$ and Gly 43 on Barstar to be critical residues in blocking the RNase activity. Mainly the $\mathrm{Asp}_{39}$ sidechain seems to mimic the interaction between Barnase and an RNA molecule as the electrostatic interactions are highly similar when comparing the crystal structures of Barnase in complex with RNA or Barstar (Schreiber, 2001). The interaction between Barstar and Barnase is primarily driven by hydrogen 
bonding utilizing $\operatorname{Arg}_{83}, \operatorname{Arg}_{87}$ and $\mathrm{His}_{102}$ sidechaines on Barnase and $\mathrm{Asp}_{39}$ on Barstar (see figure 1.7).

\subsection{Aim of this study}

Eukaryotic translation is a highly complex multistep process composed of translation initiation, elongation, termination and ribosome recycling. In total 47 individual proteins function during this process, the majority however acts during translation initiation, the most regulated and thus rate limiting step. Most translation initiation factors consist of several individual subunits, the prime example being eIF3 containing 13 different proteins. In this study we aim to analyze the wheat eIF3 complex in more detail, using biochemical approaches to in vitro reconstitute the factor from recombinantly expressed and purified subunits, thereby mapping the subunit interactions within the complex. In addition, we will analyze the native wheat eIF3 complex for so far unidentified plant specific subunits or assembly factors. By using an in vitro translation assay based on the in-house produced wheat germ extract, we aim to categorize the eIF3 subunits, but also the other wheat translation initiation factors for their functional or regulatory role during overall translation. Further we want to address the question, if the translation efficiency of an in vitro translation assay is lower as compared to the in vivo situation due to possible limitations of rate limiting translation factors.

Recent findings showed that almost all translation (initiation) factors can be found in the cargo ranges of at least one nuclear export factor, specific proteins, that shuttle their cargoes from the nuclear interior to the cytoplasm. Using an in vitro translation assay based on the rabbit reticulocyte lysate, we want to analyze if nuclear export factors, mainly Exportin 4 and Crm1 can act as compartment specific inhibitors of translation, thereby providing an additional piece of evidence weakening the hypothesis of nuclear translation.

Furthermore, we want to develop a new, beneficial set of tools for efficient, one-step affinity chromatography based on a MADA activated solid phase and the novel affinity tag system Barnase:Barstar. Therefore, the RNase Barnase must be detoxified by site-directed mutagenesis of the catalytically active amino acids. In parallel, mutations with compensatory effects must be incorporated to Barstar to preserve the stability between the two proteins. Due to the high binding affinity of the two proteins paired with their monomeric occurrence and small size, this tag system has the potential to replace streptavidin:biotin as high affinity interaction system in a broad range of applications ranging from protein purification to high-throughput screenings. 


\section{Results}

\subsection{Characterization of recombinant and native wheat elF3 complex}

Eukaryotic translation initiation factor 3 (eIF3) is the largest and most complex eukaryotic translation factor. It is comprised of 13 individual subunits ranging from 30 to $140 \mathrm{kDa}$ in size and is involved in various steps during translation initiation, e.g. bridging the $43 \mathrm{~S}$ PIC and the eIF4F complex and thus recruiting the mRNA to the ribosome (Méthot et al., 1996).

So far, most studies on eIF3 complex were either performed with the yeast complex, which only consists of 5 subunits (Asano et al., 1998), or with native complexes obtained from tissue or cultured human cells, e.g. in structural studies by Siridechadilok et al. (2005). Recently, the mammalian eIF3 complex was reconstituted using multiple polycistronic plasmids that were expressed in insect cells (Masutani et al., 2007) or in E.coli (Sun et al., 2011). However, essential questions on how the complex assembles, the interactions within the complex and also the interactions between individual eIF3 subunits and other translation factors still remain unclear.

A stepwise assembly of the eIF3 complex with individually expressed and purified subunits would greatly increase the understanding of complex assembly and - even further - would allow to analyze the effect of certain mutations or deletions on the complex formation. Furthermore, a recombinant eIF3 complex in hand or even subcomplexes could be used as add-back for depletion experiments in in vitro translation assays.

In this study, the eIF3 complex from the wheat species Triticum aestivum was analyzed. The decision towards the plant complex was driven by two main reasons: (1) to analyze the effects of individual initiation factor (sub-) complexes on overall translation rates, the wheat germ extract was used as an assay system from which certain factors can be depleted by specific antibodies. In addition, recombinant factors from the same species can be used for add-back experiments. (2) the interactions within a multimeric complex are often formed by hydrophobic interactions, thus, when the individual proteins are expressed in isolation these hydrophobic patches are suddenly solvent exposed and thus 
prone to aggregate. This is the main reason why expression of such subunits is extremely challenging and by using a wheat species with the ability to adapt to a broad temperature range in its natural habitat, different expression conditions can be screened with the aim to find conditions in which the individual subunits can stably fold.

\subsubsection{All elF3 subunits can be expressed and purified in a recombinant manner}

Successful protein expression depends on various parameters, including the expression temperature, the duration of expression, the E.coli expression strain, the induction strength, but also the N-terminal fusion tags. To determine the optimal set of parameters for each subunit, the proteins were cloned into eight different vector backbones, yielding fusion proteins with the following N-terminal tags: $\mathrm{H}_{14}, \mathrm{H}_{14}$-TEV, $\mathrm{H}_{14}$-brSUMO, ZZ-TEV, $\mathrm{H}_{10^{-}}$ ZZ-TEV, $\mathrm{H}_{14}$-shGFP-brSUMO, $\mathrm{H}_{14}$-MBP-TEV and $\mathrm{H}_{14}$-MBP-brSUMO. $\mathrm{H}_{14^{-}}$and ZZtag are well established affinity tags, binding either complexed $\mathrm{Ni}^{2+}$ ions or IgGs. TEV (Tobacco Etch Virus) and brSUMO are protease cleavage sites, which are routinely used for tag - free protein purification (Frey and Görlich, 2014a). shGFP (super hydrophilic GFP) and MBP (maltose binding protein) are rather large tags with a molecular weight of $\sim 40 \mathrm{kDa}$ and a stable fold. MBP itself is known to show chaperone-like qualities when fused to the N-terminus of a protein (Pryor and Leiting, 1997; Smyth et al., 2003).

Table 2.1: elF3 subunit solubility greatly depends on N-terminal fusion tag

All 13 eIF3 subunits were fused to different N-terminal tags. The constructs were expressed at $18^{\circ} \mathrm{C}$ over night in E.coli Top10F' cells or E.coli NEB Express cells, induced with $100 \mu$ M IPTG. The cultures were harvested, lysed and ultracentrifuged. The supernatant after ultracentrifugation was analyzed by SDS-PAGE for soluble protein. The solubility of the expressed constructs was rated from insoluble (red) over low solubility (orange), moderate solubility (yellow) to high solubility (green), meaning almost 100\% of the expressed protein was produced in a soluble form.

\begin{tabular}{|c||c|c|c|c|c|c|c|c|}
\hline & $\mathrm{H}_{14^{-}}$ & $\mathrm{H}_{14^{-T E V}}$ & $\begin{array}{c}\mathrm{H}_{14-} \\
\text { brSUMO }\end{array}$ & ZZ-TEV- & $\begin{array}{c}\mathrm{H}_{10}-\mathrm{ZZ}- \\
\text { TEV }\end{array}$ & $\begin{array}{c}\mathrm{H}_{14^{-}} \\
\text {shGFP- } \\
\text { brSUMO }\end{array}$ & $\begin{array}{c}\mathrm{H}_{14} \text {-MBP- } \\
\text { TEV }\end{array}$ & $\begin{array}{c}\mathrm{H}_{14} \text {-MBP- } \\
\text { brSUMO }\end{array}$ \\
\hline \hline eIF3a & insoluble & insoluble & insoluble & insoluble & insoluble & insoluble & low & low \\
\hline eIF3b & moderate & high & high & moderate & moderate & moderate & high & moderate \\
\hline eIF3c & high & high & high & high & high & high & high & high \\
\hline eIF3d & moderate & high & high & high & moderate & high & high & high \\
\hline eIF3e & insoluble & insoluble & insoluble & moderate & low & moderate & moderate & high \\
\hline eIF3f & insoluble & insoluble & insoluble & moderate & low & high & high & high \\
\hline eIF3g & insoluble & insoluble & moderate & moderate & insoluble & high & moderate & high \\
\hline eIF3h & moderate & moderate & low & moderate & low & moderate & high & high \\
\hline eIF3i & insoluble & insoluble & insoluble & moderate & insoluble & insoluble & moderate & low \\
\hline eIF3j & moderate & high & high & high & high & high & high & high \\
\hline eIF3k & high & high & high & high & moderate & high & moderate & high \\
\hline eIF3l & insoluble & insoluble & insoluble & insoluble & insoluble & insoluble & low & low \\
\hline eIF3m & insoluble & insoluble & insoluble & insoluble & low & moderate & high & high \\
\hline
\end{tabular}

The constructs were transformed into E.coli Top10F' cells and expressed over night at $18^{\circ} \mathrm{C}$. Expression was induced by the addition of $100 \mu \mathrm{M}$ IPTG, which releases repression by lacI, thereby enabling transcription of the downstream genes. The cells were subsequently harvested, resuspended in buffer $(50 \mathrm{mM}$ Tris $/ \mathrm{HCl} \mathrm{pH} 7.5,500 \mathrm{mM} \mathrm{NaCl}, 5 \mathrm{mM} \mathrm{MgAc}, 1 \mathrm{mM}$ DTT), lysed by sonication and ultracentrifuged. Soluble protein is expected to remain in the supernatant after centrifugation, whereas protein aggregates precipitate to the pellet. 
The supernatant samples were analyzed by SDS-PAGE to estimate the solubility of each construct. The results are summarized in table 2.1.

The solubility of eIF3 subunits was also screened at higher temperatures and shorter expression times $\left(23^{\circ} \mathrm{C}, 6 \mathrm{~h}\right)$. Although some eIF3 subunits such as eIF3c, eIF3d and eIF3j could be expressed in a soluble manner, the majority of the subunits tends to express better when induced at lower temperatures for a longer time period (data not shown).

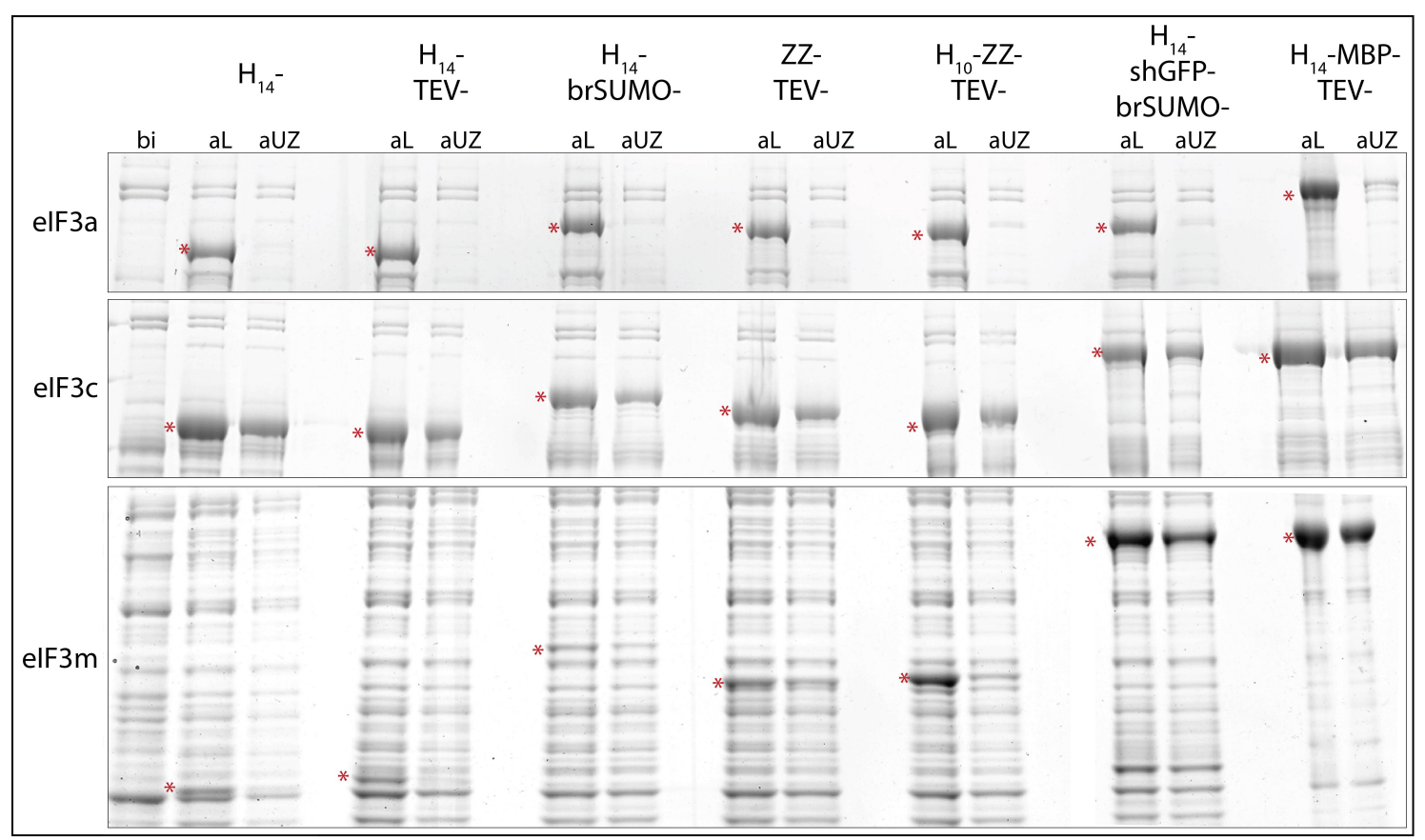

Figure 2.1: Solubility of elF3 subunits greatly depend on N-terminal fusion tags

eIF3 subunits eIF3a, eIF3c and eIF3m were expressed as fusion constructs with various N-terminal affinity tags. Expression was performed in E.coli TOP10F' cells at $18^{\circ} \mathrm{C}$ over night with $100 \mu \mathrm{M}$ IPTG (for details see 4.2.2.3). Samples were taken after lysis of the cells by sonication $(\mathrm{aL})$ and from the supernatant after ultracentrifugation (aUZ). Soluble protein should remain in the supernatant whereas insoluble proteins should have gone to the pellet. As control, non-induced E.coli cells were loaded as well (bi). (*) indicates the expressed protein bands. All samples were loaded at a concentration of $30 \mathrm{mOD} /$ lane on a polyacrylamide gradient gel and stained with Coomassie. shGFP: super hydrophilic GFP variant

In general, one can observe an order of solubility (1) between the individual eIF3 subunits and (2) between the different affinity tags used. When analyzing the solubility of the eIF3 subunits under all tested conditions, subunits eIF3b, eIF3c, eIF3d, eIF3j, and eIF3k are soluble with any tag (see table 2.1 and figure 2.1 eIF3c), indicating a stable fold of the individual proteins. eIF3h behaves similar to those subunits, however in comparison, expression and solubility levels are slightly lower. Subunits eIF3e, eIF3f, eIF3g, eIF3i, and eIF3m can not be expressed in a soluble manner with small tags, but the soluble fraction increases with increasing tag size (see 2.1 eIF3m). Subunits eIF3a and eIF3l show severe solubility problems. Both can only be expressed as partly soluble proteins when fused to MBP (see 2.1 eIF3a).

When grading the affinity tags for their ability to increase solubility of their fusion partners, 
the rule of thumb seems to be: the larger the fusion tag, the more soluble protein can be produced (see figure 2.1 eIF3m and table 2.1). However, there are some exceptions. When comparing ZZ-TEV and $\mathrm{H}_{10}$-ZZ-TEV, the slightly larger $\mathrm{H}_{10}$-ZZ-TEV tag is not able to positively influence the solubility of e.g. eIF3g or eIF3i, whereas ZZ-TEV-fusion proteins show a moderate solubility. Possibly the biochemical properties of the histidine sidechains interfere with the folding of these particular eIF3 subunits. Another interesting phenomena is that although the $\mathrm{H}_{14}$-shGFP-brSUMO, $\mathrm{H}_{14}$-MBP-TEV and $\mathrm{H}_{14}$-MBP-brSUMO tags are all similar in size, only fusions to MBP enable to produce eIF3a and eIF31 in a slightly soluble manner. This might be explained by the chaperone-like qualities of MBP.

These screenings show that affinity tags can enhance the solubility of their fusion partners and that there seems to be a correlation between tag size and soluble fraction of the expressed protein. However, it also shows that the ideal tag for each protein has to be individually determined to perfectly match and positively influence the biochemical properties and folding kinetics of the protein.

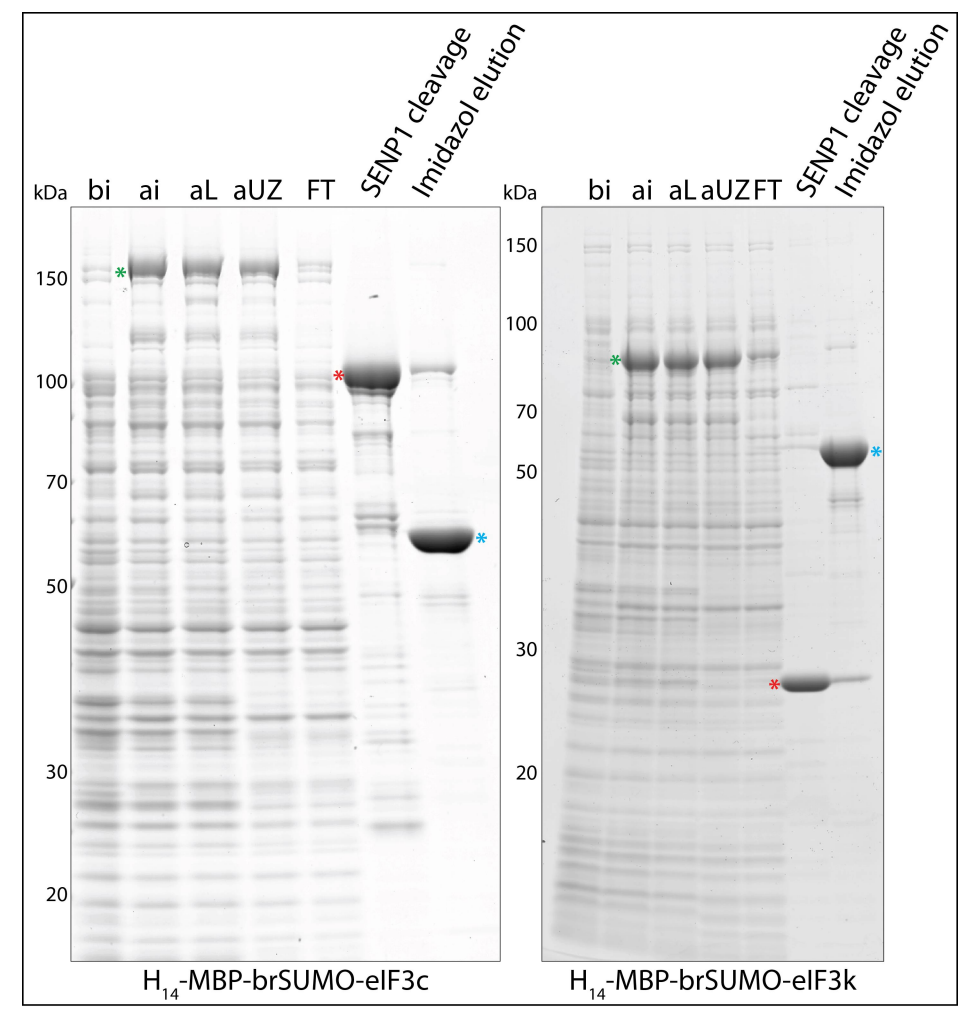

Figure 2.2: Purification of elF3 subunits by protease elution

eIF3 subunits eIF3c and eIF3k were N-terminally fused to an $\mathrm{H}_{14}$-brSUMO-MBP tag and expressed in E.coli NEB Express cells at $18^{\circ} \mathrm{C}$ over night with $200 \mu \mathrm{M}$ IPTG (for details see 4.2.2.3). Samples were taken before induction $(\mathrm{bI})$, after induction (aI), after lysis by sonication $(\mathrm{aL})$ and from the supernatant after ultracentrifugation (aUZ). The lysate was applied to $6 \% \mathrm{Ni}^{2+}$-chelate $500 \AA$ silica and after binding in batch for $1 \mathrm{~h}$, the flow through (FT) was collected. The resin was thoroughly washed and incubated with $0.1 \mu \mathrm{M}$ SENP1 protease cutting at the brSUMO protease recognition site. After incubation for $1 \mathrm{~h}$, the cut protein was washed out. As control, the remaining protein on the matrix was eluted with $1 \mathrm{M}$ imidazole. The full length construct is marked with green asterisks, the cleaved protein with red asterisks and the remaining tag is marked with light blue asterisks. All samples were loaded at a concentration of $30 \mathrm{mOD} /$ lane on a polyacrylamide gradient gel and stained with Coomassie. 
In summary, expression conditions yielding in soluble proteins could be determined for each of the 13 eIF 3 subunits, allowing to further purify the subunits by affinity chromatography as shown in figure 2.2 exemplarily for subunits eIF3c (left panel) an eIF3k (right panel). The proteins were expressed as $\mathrm{H}_{14}$-MBP-brSUMO constructs and elution was performed by on-column protease cleavage using brSENP1 protease (purification scheme can be seen in figure 1.5). Due to cleavage, the eIF3 subunit looses its affinity to the matrix and elutes, whereas the remaining tag (here $\mathrm{H}_{14}$-MBP-brSUMO) remains bound to the beads and can only be released from the $\mathrm{Ni}^{2+}$-beads with high imidazole concentrations, which displaces the His-tag and thereby the bound protein.

\subsubsection{In vitro reconstitution of recombinant elF3}

The successful expression and purification of all 13 wheat eIF3 subunits as described in section 2.1.1 was an important step towards the recombinant reconstitution of the entire complex. Consequently, binding assays were performed to map the stepwise assembly of the subunits during formation of the $\sim 800 \mathrm{kDa}$ complex. All interaction studies were performed on commercial IgG beads, specifically binding ZZ-tagged proteins (for experimental details see 4.2.3). First, eIF3 subunits were analyzed pairwise for possible interactions. Thereby, one protein was ZZ-tagged, the other protein was either tag free or contained a histidine-tag. Both were mixed in E.coli lysate $(50 \mathrm{mM}$ Tris/ $\mathrm{HCl} \mathrm{pH} 7.5,500 \mathrm{mM} \mathrm{NaCl}$, $1 \mathrm{mM}$ DTT) and subsequently applied to the beads. After incubation, the flow through was collected, the beads were washed and the bound fraction was eluted by SDS-loading buffer. As control, IgG beads were incubated with E.coli lysates containing only the subunits without ZZ-tag to analyze its background binding. This screening did not result in any observed interactions between two subunits, possibly indicating that a stable interaction between the subunits requires more than two subunits. Hence, various combinations were analyzed, such as the five subunits homologous to the yeast eIF3 complex, the eight subunits resembling the eIF3 core complex in humans (see section 1.1.1), or all 13 eIF3 subunits. Surprisingly, in neither of the combinations interactions between two or more subunits was observed. A possible explanation would be that the assembly of the wheat eIF3 complex requires certain eukaryotic assembly factors or processing enzymes that lack in E.coli. Thus, the experiments were repeated, now screening for interactions in the presence of the wheat germ extract instead of the E.coli lysate. However, also these trails were not successful.

In 1983, the term "molten globule" was coined (Ohgushi and Wada, 1983), describing a partially folded protein state in which the polypeptide chain is packed similarly as in the native state, however the protein interior is not (yet) tightly packed and intramolecular interactions are not stably formed. This folding state, that can be either relieved by 
chaperones, respectively, or form a stable non changeable intermediate, might explain the phenomena that the eIF3 subunits can be expressed in a soluble manner however they fail in interacting with their natural binding partners. To avoid the formation of potential molten globule proteins and to further increase solubility of the subunits by providing possible interaction partners, various eIF3 subunits were co-expressed either in pairs or triples. Therefore each protein was encoded on plasmids with different origin of replications and selection markers. Co-expression revealed that indeed solubility of so far less soluble subunits could be increased. Interestingly, this effect seems to be specific, as not every subunit can help another to fold more stable. Exemplarily, solubility of eIF3f can be positively influenced when co-expressed with eIF3b, co-expression of eIF3c however does not have any effect on eIF3f. Co-expressed proteins were further analyzed for their ability to be co-purified (only one protein was fused to a ZZ-tag), however, possible interactions seem to be too weak to enrich both proteins on a resin. Co-expression of multiple tandem vectors encoding all eIF3 subunits, similar to the approach taken by Masutani et al. (2007) could enable to produce an entire eIF3 complex in E.coli avoiding misfolded or aggregated proteins.

Note that during the purification of eIF3b, a $\sim 60 \mathrm{kDa}$ protein co-purified, which was identified by mass spectrometry to be the E.coli GroEL chaperone. This might indicate folding problems of the subunit although it results in highly soluble protein.

\subsubsection{Characterization of native elF3 in the Wheat Germ Extract}

Although all 13 eIF3 subunits could be expressed in E.coli and further purified, in vitro reconstitution was not successful. Possibly, eIF3 assembly requires additional factors or the plant eIF3 complex is comprised out of further subunits so far unknown. To address these questions, the native wheat eIF3 complex was analyzed in immunoprecipitation (IP) experiments and the wheat germ extract was fractionated in order to analyze either the whole eIF3 complex or sub complexes by Western Blot.

Both techniques require antibodies, which specifically recognize the wheat eIF3 subunits. Thus, in corporation with Dr. Cathrin Enke, antibodies against all wheat eIF3 subunits were raised and purified. High yields of antibodies were obtained due to the immunogenic potential of wheat proteins.

\subsubsection{Purification and characterization of antibodies against elF3}

Individually expressed and purified eIF3 subunits were used to immunize rabbits, thereby inducing the production of antibodies against the target proteins in the animal. The antibodies were purified from the rabbit sera using multiple columns containing different proteins to reduce the amount of cross-reacting antibodies in the final elution: (1) E.coli 
lysate coupled column to remove antibodies recognizing potential co-purified and thus coinjected E.coli proteins, (2) affinity tag coupled column to remove antibodies recognizing the tag of the fusion-protein injected into the rabbits (here $\mathrm{H}_{14}$-MBP-TEV) tag, (3) antigen coupled column to accumulate antibodies recognizing the target protein. Instead of the entire target protein, also individual domains can be used as antigen. Bound antibodies were eluted by $0.1 \mathrm{M}$ glycine $\mathrm{pH} 2.2$, neutralized and precipitated with ammonium sulphate. For analysis, precipitated antibodies were dissolved in 2xPBS buffer and a Western Blot was performed on wheat germ extract stripes using $1 \mu \mathrm{g} / \mathrm{ml}$ of the purified antibodies (figure $2.3 \mathrm{~A}$ ). As shown, antibodies against eIF3h, eIF3i, eIF3l and eIF3m are highly specific towards their target protein and do not show any cross-reactions to other proteins present in the wheat germ extract. Antibodies against eIF3e, eIF3f, eIF3g, eIF3j and eIF3k do show cross-reactivity, however the main signal correlates with the target protein.

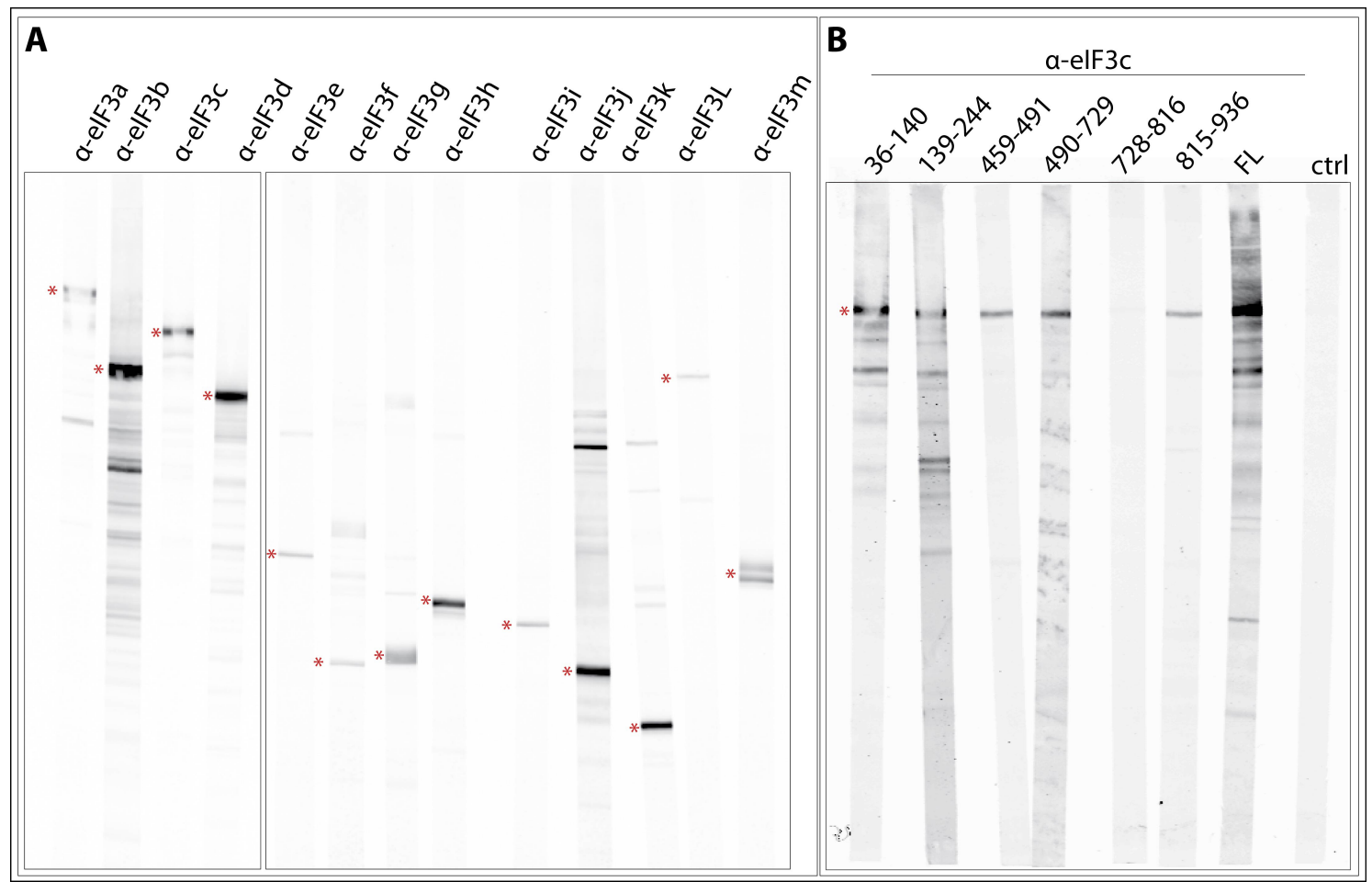

Figure 2.3: Purification of antibodies against elF3 subunits

(A) Antibodies against all 13 wheat eIF3 subunits were purified from rabbit sera as described in 4.2.5.2. (B) antibodies recognizing wheat eIF3c were further purified via individual eF3c domains to increase the sensitivity of the antibodies towards the target protein and to remove cross-reacting antibodies. Analysis was performed by Western Blot on membrane stripes containing wheat germ extract. After blocking the membrane, each stripe was incubated with $1 \mu \mathrm{g} / \mathrm{ml}$ of the purified antibody. After over night incubation and thorough washing with $1 x T B S$, the secondary goat $\alpha$-rabbit IRdye antibody was added (1:50.000) for $1 \mathrm{~h}$. After washing, the blot was analyzed using the Odyssey scanner (settings: $700 \mathrm{~nm}$ (L2.0, 800nm (1.0))). FL: full length; ctrl: control membrane: the wheat germ extract containing stripe was incubated with the secondary antibody only.

Antibodies against the four largest eIF3 subunits eIF3a, eIF3b, eIF3c and eIF3d show cross-reactivity to a multitude of other proteins, either caused by degradation products of the target protein or by similar epitops in other wheat proteins. To reduce the amount 
of cross-reactions, the antibodies were subsequently purified via individual domains of the target proteins. For analysis, wheat germ extract was blotted on nitrocellulose, cut into stripes and incubated with the domain specific antibodies. Figure 2.3 B exemplarily shows the purification of the eIF3c antibodies against individual domains of the wheat eIF3c proteins. Antibodies recognizing domains eIF3c459-491 and eIF3c815-936 are highly specific towards eIF3c and do not show any other cross reactivity whereas antibodies purified via the full length eIF3c proteins shows significant cross-reactivity with other proteins present in the extract.

Optimization of antibodies against eIF3a, eIF3b, eIF3d and eIF3j were performed as well. This work was greatly supported by the lab rotation students Metin Aksu and Ayesha Khan.

\subsubsection{2 elF3 forms a stable complex in the wheat germ extract}

To analyze the composition of the native wheat eIF3 complex, the in-house prepared wheat germ extract was rebuffered from a low salt HEPES based buffer system to $50 \mathrm{mM}$ Tris/HCl pH7.5, 500mM NaCl, $5 \mathrm{mM} \mathrm{MgAc}, 1 \mathrm{mM}$ DTT and fractionated using a SD200 16/60 gel filtration column (Pharmacia).

The eluted fractions were loaded on SDS polyacrylamide gels and analyzed for their eIF3 subunit content by Western Blot using the previously described eIF3 specific antibodies (see figure $2.3 \mathrm{~A}$ ). Figure 2.4 shows the gelfiltration profile and a schematic representation of the tested eIF3 subunit content in each fraction. eIF3b, eIF3c, eIF3m, eIF3h and eIF3k seem to form a stable complex in the wheat germ extract as they co-migrate through the column. Members of all described eIF3 subcomplexes (Zhou et al., 2008) were tested in Western Blot suggesting that also the additional, non-tested eIF3 subunits migrate similarly. eIF3j is an exceptional eIF3 subunit due to the fact that it only associates loosely to the complex (Fraser et al., 2004). Hence, it eluted later from the column. As control eIF $2 \alpha$ as non-eIF3 subunit was tested as well showing that this subunit did not co-migrate with the tested eIF3 subunits but eluted later from the column.

To further validate that eIF3 forms a stable complex in the wheat germ extract and to identify possible additional subunits or assembly factors, immunoprecipitation experiments were performed. Recombinantly expressed and purified ProteinA was coupled to maleimide 4B Sepharose (produced in the lab by D. Görlich) via a reduced cystein at the C-terminus of the protein. Next, IgGs against eIF3 subunits were covalently attached and the beads were incubated with wheat germ extract rebuffered to $50 \mathrm{mM}$ Tris/ $\mathrm{HCl} \mathrm{pH} 7.5$, $500 \mathrm{mM} \mathrm{NaCl}, 5 \mathrm{mM} \mathrm{MgAc}, 1 \mathrm{mM}$ DTT and centrifuged to remove aggregates. After incubation, the flow-through was collected and the beads were washed. Bound proteins were eluted with $0.1 \mathrm{M}$ glycine $\mathrm{pH} 2.2$ and subsequently precipitated with trichloroacetic acid. 


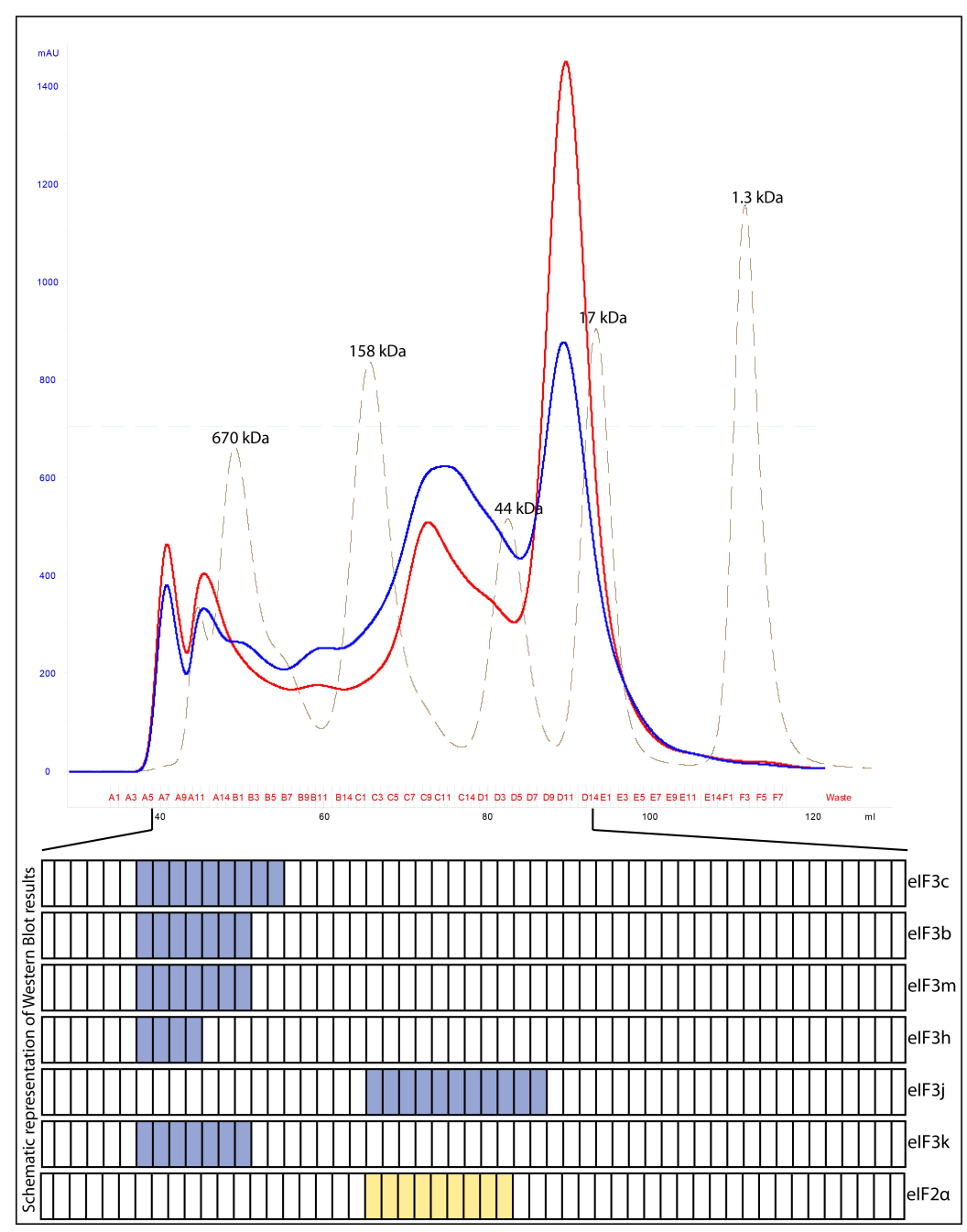

Figure 2.4: Native elF3 forms a stable complex in the WGE

Wheat germ extract was rebuffered to $50 \mathrm{mM}$ Tris/HCl pH7.5, $500 \mathrm{mM} \mathrm{NaCl}, 5 \mathrm{mM} \mathrm{MgAc}, 1 \mathrm{mM}$ DTT and subsequently pre-cleared by ultracentrifugation. The supernatant was loaded onto a SD200 16/60 gel filtration column with a constant flow of $1 \mathrm{ml} / \mathrm{min}$. For size estimation $0.5 \mathrm{ml}$ Gel filtration standard (BioRad) was loaded to the column under the same conditions. $1 \mathrm{ml}$ elution fractions were collected and analyzed for their eIF3 subunit content by Western Blot (antibodies used at $1 \mu \mathrm{g} / \mathrm{ml}$ concentration). The distribution of eIF3 subunits in the individual fractions is shown schematically, eIF $2 \alpha$ distribution is shown in yellow.

Elution samples were loaded onto polyacrylamide gels, stained with Colloidal Coomassie, the resulting bands were cut and finally analyzed by mass spectrometry to identify the immunoprecipitated proteins. Initial experiments resulted in elution fractions containing many proteins binding nonspecifically to the resin. To improve the purity of the immunoprecipitation elution samples a pre-clearing step of the wheat germ extract via ProteinA beads without coupled IgGs was performed, which greatly improved the purity of the samples.

Figure 2.5 exemplarily shows the elution samples for immunoprecipitation experiments performed with antibodies against wheat eIF3b and eIF3c. The proteins eluted from the beads used for pre-clearing can be seen as well (pre-clearing), showing the vast amount 
of proteins unspecifically binding to the resin. The bands in the IP elution fractions are labeled according to the mass spectrometry results, the direct target protein of the used antibody is highlighted in red. In both cases all eIF3 subunits except the loosely attached eIF3j subunit co-precipitated with the target protein, strongly arguing for a stable eIF3 complex in the wheat germ extract. In addition, elongation factor eEF1 $\alpha$ was identified in both cases, the chaperone CPN60 was found as a major band in the eIF3b IP sample. It remains to be elucidated if the chaperone is a complex constituent or co-precipitated due to cross-reactivity of the antibody.

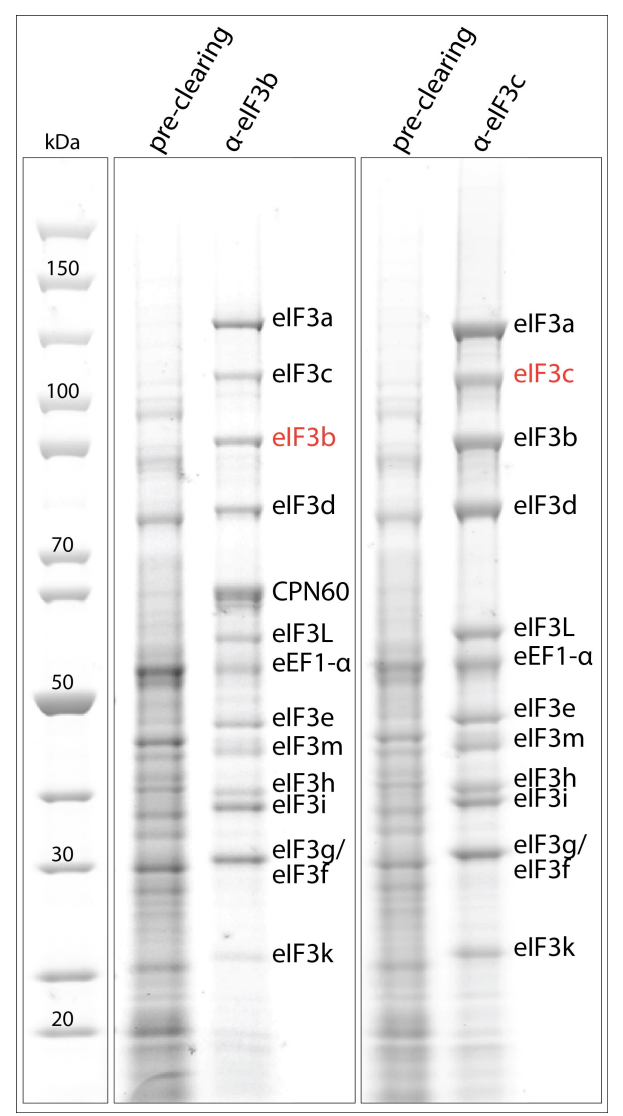

Figure 2.5: Identification of the native wheat elF3 complex

To identify the components of the native wheat eIF3 complex, immunoprecipitation experiments were performed as described in 4.2.5.4. To achieve a higher purity of the elution, the wheat germ extract was pre-cleared over ProteinA beads not containing any coupled antibodies (bound proteins shown in "pre-clearing"). The IP experiments were performed with ProteinA beads covalent attached to either $\alpha$-eIF3b or $\alpha$-eIF3c antibodies. Elution samples were loaded on $12 \%$ polyacrylamid gels and stained with colloidal Coomassie. The resulting bands were analyzed by Mass Spectrometry revealing all eIF3 subunits co-precipitating except eIF3j.

Both, the wheat germ extract fractionation and the IP experiments showed that the native eIF3 complex in the wheat germ extract is present in a stable form. All 13 proposed plant eIF3 subunits could be identified by mass spectrometry. The aim to find possible additional eIF3 subunits or factors being attached to the complex and thereby stabilizing it, was not successful. As already mentioned, the complex could not be reconstitution by mixing individual recombinant subunits, although these seem to be sufficient to form a stable 
and functional complex in vivo. This once more strongly suggests that the recombinantly produced eIF3 subunits are folding intermediates. They can be expressed in a soluble form but are nevertheless non- or misfunctional proteins and thus can not assemble in vitro.

\subsection{Use of in vitro translation systems to reveal limitations of and necessities for individual translation factors}

Translation is a highly complex process involving 47 individual proteins in higher eukaryotes. Thereby translation initiation requires most of the factors, all together 37 individual proteins. Some function singly, e.g. eIF1, some act as multimeric complexes, e.g eIF3, eIF2B or eIF2 complex. By looking at these complex factors and comparing their composition between yeast and higher eukaryotes, a striking difference in complexity can be observed, raising the question if all higher eukaryotic subunits are truly essential for a proper translation or if there are functional and regulatory subunits. However, these questions are difficult to access in in vivo systems as translation is a fundamental process in every living organism and depleting or manipulating functional or regulatory subunits could lead to an overall decrease of translation and thus lethality. Hence, it is highly advantageous to analyze the necessity of various translation (initiation) factors in context of an in vitro translation assay. Another interesting observation is that although the wheat germ extract is the most efficient higher eukaryotic in vitro translation system, the efficiency of protein synthesis compared to the in vivo situation is dramatically reduced. Most likely certain components in the in vitro systems are limiting, probably even translation (initiation) factors. To follow up this hypothesis and to analyze which translation factor subunits play a functional or regulatory role, an in vitro translation assay based on the wheat germ extract was used.

For the in vitro wheat germ translation assay, the extract is supplemented with amino acids, an energy regenerating system and mRNA coding for the reporter protein (here Firefly Luciferase). The reaction is incubated for 90 minutes at $27^{\circ} \mathrm{C}$ during which the mRNA is translated to protein. The more translation efficient the extract is, the more luciferase is produced. Next, a luminescence assay is performed, in which a luciferin solution is added to the wheat germ extract, inducing the conversion of luciferin to oxyluciferin and light. This reaction is catalyzed by the produced luciferase. Thus, by measuring the luminescence, the amount of luciferase enzyme produced during the translation reaction can be calculated. Translation (initiation) factors or factor subunits can be either titrated to the extract in a recombinant form, or they can be specifically depleted by using the previously described wheat translation factor specific antibodies. The translation efficiency 
of these supplemented or depleted extracts is then compared to the efficiency of non treated extracts.

\subsubsection{Addition of recombinant initiation factors does not stimulate translation efficiency}

Extensive work by Dr. C. Enke showed that following optimizations can greatly increase the translation efficiency of the in vitro wheat germ translation system (Enke, 2010):

- addition of translation enhancing elements to the in vitro translation vector

- optimization of extract preparation

- optimization of translation conditions

- usage of winter wheat grains as starting material

However, although it performs $\sim 8$-fold better than commercially available systems, the translation efficiency is still significantly low compared to the corresponding in vivo system. If one wants to elevate the in vitro wheat germ translation system to a biotechnological tool that can be used for the production of high protein amounts, the limitations preventing highly efficient translation need to be determined and abolished. The wheat germ extract is produced from translationally inactive embryos, which nevertheless should contain all relevant translation factors to be able to start protein synthesis once required. However, the amount of translation factors might be sufficient during early plant development but be rate-limiting in the in vitro assay. If this is the case, addition of translation factors to the translation assay should lead to an increased production of firefly luciferase.

Recombinantly expressed and purified translation factors fused to $\mathrm{H}_{14}$-MBP-brSUMO were titrated to the translation assay in concentrations ranging from $0 \mu \mathrm{M}$ to $1 \mu \mathrm{M}$. To ensure a sufficient cleavage of the fusion construct, 40nM SENP1 protease was additionally added to support the endogenous SUMO protease. Addition of the recombinant protease to the extract does not affect translation efficiencies. The amounts of produced Firefly luciferase in the supplemented extracts was measured, calculated and subsequently compared to the rates obtained from non-supplemented extracts. In the first trails, 16 wheat translation initiation factor subunit were titrated individually to the extract, resulting in no significant improvement of the translation efficiency. Slight increases in translation rates were observed when members of the mRNA cap-binding eIF4F complex were added. Hence, combinations of eIF4A, eIF4E, eIF4isoE, eIF4G and eIF4isoG were subsequently tested, however the first results could not be reproduced.

Obviously, addition of recombinant translation factors was not able to increase the translation efficiency of the extract. One possible explanation is that the recombinantly expressed 
and purified subunits are not functional in the assay. Although all factors were expressed as MBP fusion constructs, thereby potentially being positively influenced by the tags chaperone-like qualities, the folding could still result in non-functional or misfunctional intermediates or the factors aggregate upon tag removal in the extract. Another explanation refers to the tag removal itself. Although the SUMO protease is known to be highly efficient, cleavage might take too long and the attached large tag could meanwhile hinder the factor to incorporate into multi-factor complexes acting during translation. Alternatively, the obtained data could also indicate that translation factors are not the limiting compounds in the extract, but other parameters such as energy supply and mRNA stability need to be further analyzed.

\subsubsection{Depletion of elF3 from wheat germ extracts leads to a reduced translation efficiency}

Even if the addition of translation factors to the extract does not positively affect the translation rates of an in vitro wheat germ translation system, removal of endogenous factors from the system could however show severe consequences. A significant decrease in translation efficiency upon depletion of a particular translation factor would strongly argue that this factor is essential during translation. Add-back of the depleted factor or subunit should be able to restore the translation efficiency. If translation rates remain constant even upon depletion of certain factors, these proteins are most likely not essential during translation. Such depletion and add-back experiments can be used to identify the minimal set of proteins required in higher eukaryotes for proper protein synthesis. This would be an important piece of information in terms of eukaryotic translation system reconstitution.

To deplete translation factors or factor subunits from the extract, the already described antibodies against eIF3 were used (see section 2.1.3.1), additionally also antibodies against GFP and translation factors eIF $2 \alpha$, eIF2 $\beta$, eIF5 and eEF1B $\beta$ (characterized in Enke, 2010). Depletion was performed in two consecutive rounds, using $500 \mu$ l wheat germ extract and $2 \mathrm{x} 50 \mu \mathrm{l}$ ProteinA maleimide beads to which the corresponding antibodies had been covalently attached. After depletion, the extracts were analyzed by Western Blot for the amount of remaining target protein and potential co-depletions. Further, the depleted extracts were used in translation assays. Therefore, the samples were supplemented with the energy regenerating system, mRNA encoding Firefly Luciferase, amino acids and in case of add-back experiments with appropriate amounts of recombinant translation factors. The translation reaction was incubated for 90 minutes at $27^{\circ} \mathrm{C}$ and subsequently the amount of produced luciferase was determined. 


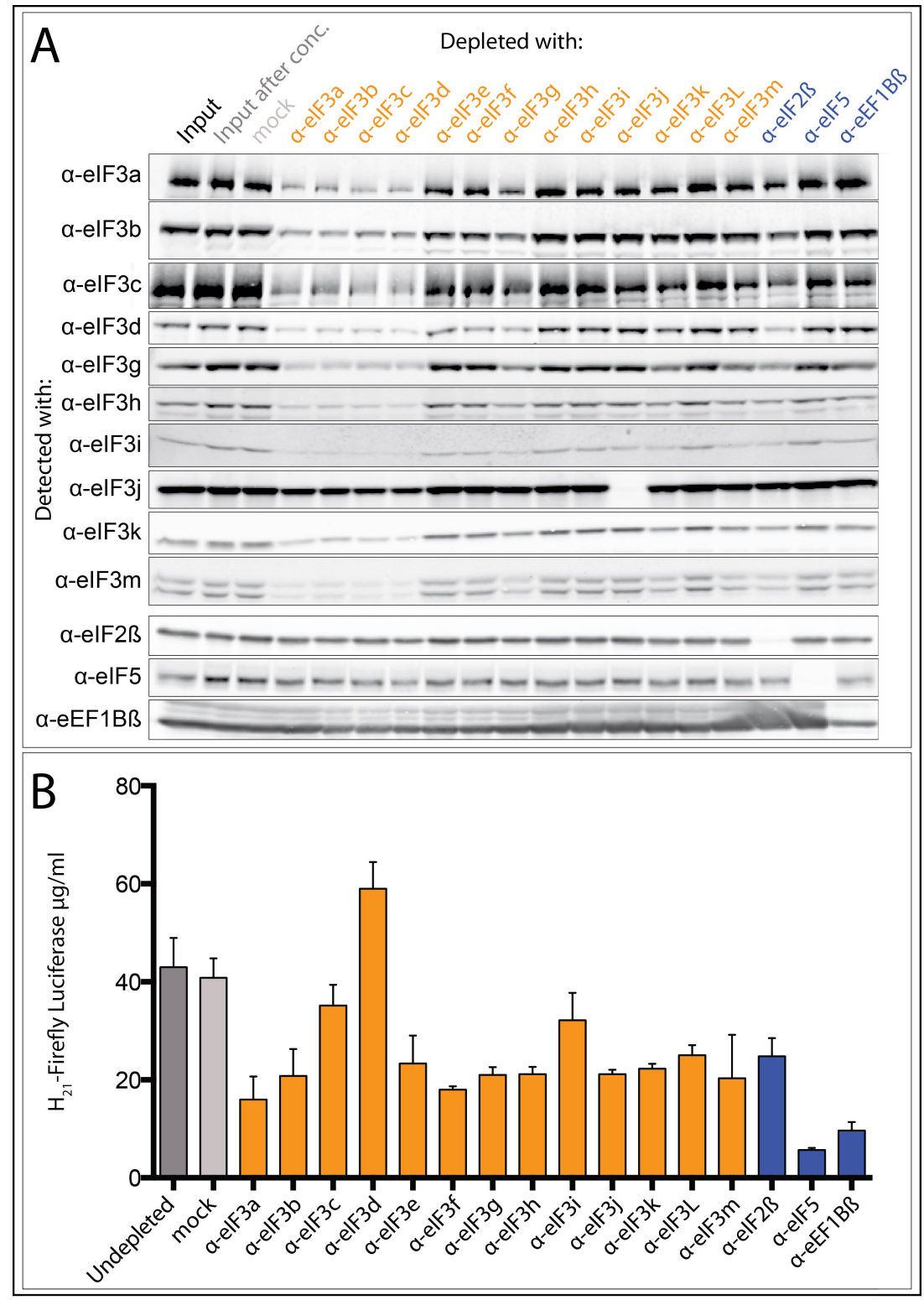

Figure 2.6: Depletion of translation initiation factors decreases overall translation efficiency

Translation initiation factors were depleted from wheat germ extract by subunit specific antibodies. (A): Depleted extracts were analyzed by Western Blot for their remaining target protein content and for possible co-precipitation of additional subunits. As control, a non-depleted extract (Input) and a mock depleted extract (depleted with $\alpha$-GFP antibodies) was analyzed in parallel. Extracts were depleted with antibodies against all 13 eIF3 subunits (orange), as well as antibodies against two further translation initiation factors and one elongation factor (blue). Primary antibodies were used at a concentration of $1 \mu \mathrm{g} / \mathrm{ml}$, secondary goat $\alpha$-rabbit IRyde antibodies were used at a concentration of 1:50.000. (B): In vitro translation assays were performed with the depleted extracts. The translation efficiency was analyzed by estimating the amount of produced Firefly luciferase during the translation reaction by performing a luciferase activity assay using the BioTek Synergy H4 Plate Reader.

First, all eIF3 subunits were individually depleted from the extract by their subunit specific antibodies. As control, a mock depletion using $\alpha$-GFP was performed as well as depletions utilizing antibodies against eIF2 $\alpha$, eIF5 and eEF1B $\beta$. The depletion efficiency was analyzed by Western Blot (figure 2.6A) comparing the depleted extracts with extracts that were not depleted (Input, Input after conc.). Detection was performed using anti- 
bodies against most eIF3 subunits, eIF $2 \alpha$, eIF5 and eEF1B $\beta$. The amount of detected proteins in the Input and mock depleted samples are comparable, indicating that the target proteins do not unspecifically bind to the antibody scaffold or to the resin. When depleting the extracts with eIF $2 \alpha$, eIF5 or $\operatorname{eEF} 1 \mathrm{~B} \beta$, a reduction of the target protein can be observed whereas all other tested proteins remain unaffected. The depletion efficiency for eIF $2 \alpha$ and eIF5 is high as only slight amounts of protein can still be detected. In case of $\operatorname{eEF} 1 \mathrm{~B} \beta$, the amount of protein in the extract also decreases, however $\sim 50 \%$ of the endogenous protein remains. When depletion was performed with antibodies against eIF3a, eIF3b, eIF3c or eIF3d, the amount of the target protein and also all other tested eIF3 subunits, except eIF3j, decreases significantly. The fact that eIF3j is only loosely attached to the native eIF3 complex explains, why no reduction of the protein in extracts depleted with core eIF3 subunits can be observed. In contrast, when depleting the extract with antibodies against eIF3j, almost no target protein can be detected any longer, whereas the other eIF3 subunits remain unaffected.

Analyzing the native wheat eIF3 complex showed that the factor seems to be present in a stable form in the extract (see figure 2.5, respectively). However, when depleting the extracts with antibodies against eIF3e-eIF3m, slight decreases in the target protein can be observed, but other subunits can not be co-depleted, and the depletion efficiency is generally very low. Note that antibodies against the eIF3 subunits were raised against individual subunits. Epitops might be shielded when the protein is incorporated into the eIF3 complex. Thus, these subunits could only be recognized and bound by the antibodies when present in a non-complexed form.

The (partially) depleted extracts were used in the in vitro wheat germ translation system, assaying for the amount of produced luciferase in comparison with non- or mock- depleted extracts (figure 2.6B). Mock depleted samples produce similar amounts of luciferase as non-treated extracts, showing that the depletion procedure itself does not affect the translation efficiency of the extract. The extracts depleted with eIF3 subunit specific antibodies did not lead to conclusive results. Even though the Western Blot analysis showed that the amounts of all tested eIF3 subunits strongly decreases upon depletion with antibodies against eIF3a, eIF3b, eIF3c and eIF3d, the resulting extracts behave very different in terms of translation efficiency. The $\alpha$-eIF3a and $\alpha$-eIF3b depleted extract show an $\sim 2$-fold decrease in translation efficiency, the extract depleted with antibodies against eIF3c produces similar amounts of protein as the non-depleted extracts and surprisingly, extracts depleted with $\alpha$-eIF3d antibodies even shows an increase in translation efficiency. On the other side, depleted extracts that only shows a minor decrease of the target protein and the other eIF3 subunits in the Western Blot analysis show a strong reduction of translation efficiency, e.g. when using antibodies eIF3g or eIF3h. 
Depletion of eIF $2 \beta$ leads to an $\sim 2$-fold reduction of the produced protein, the effects upon eIF5 and eEF1B $\beta$ depletion however are more drastic. eIF5 is the GTPase activating protein for the eIF2 complex. GTP hydrolysis in eIF2 leads to the stable accommodation of the initiator tRNA to the P-site of the small ribosomal subunit. Interestingly, depletion of eIF5 seems to strongly affect the translation efficiency of the extract, however, depletion of eIF2 $\beta$, a component of the eIF2 complex shows milder effects. eEF1B $\beta$ is part of the heterotrimeric eEF1B complex, a translation elongation factor acting as nucleotide exchange factor (GEF) for eEF1A. It plays an important role in recruitment of aminoacyltRNAs onto the ribosome (Janssen and Moller, 1988). Thus, even a slight decrease in the amounts of endogenous protein could hinder eEF1A to participate in a new round of tRNA delivery to the ribosome, which leads to an overall delay in protein synthesis and hence less produced reporter protein.

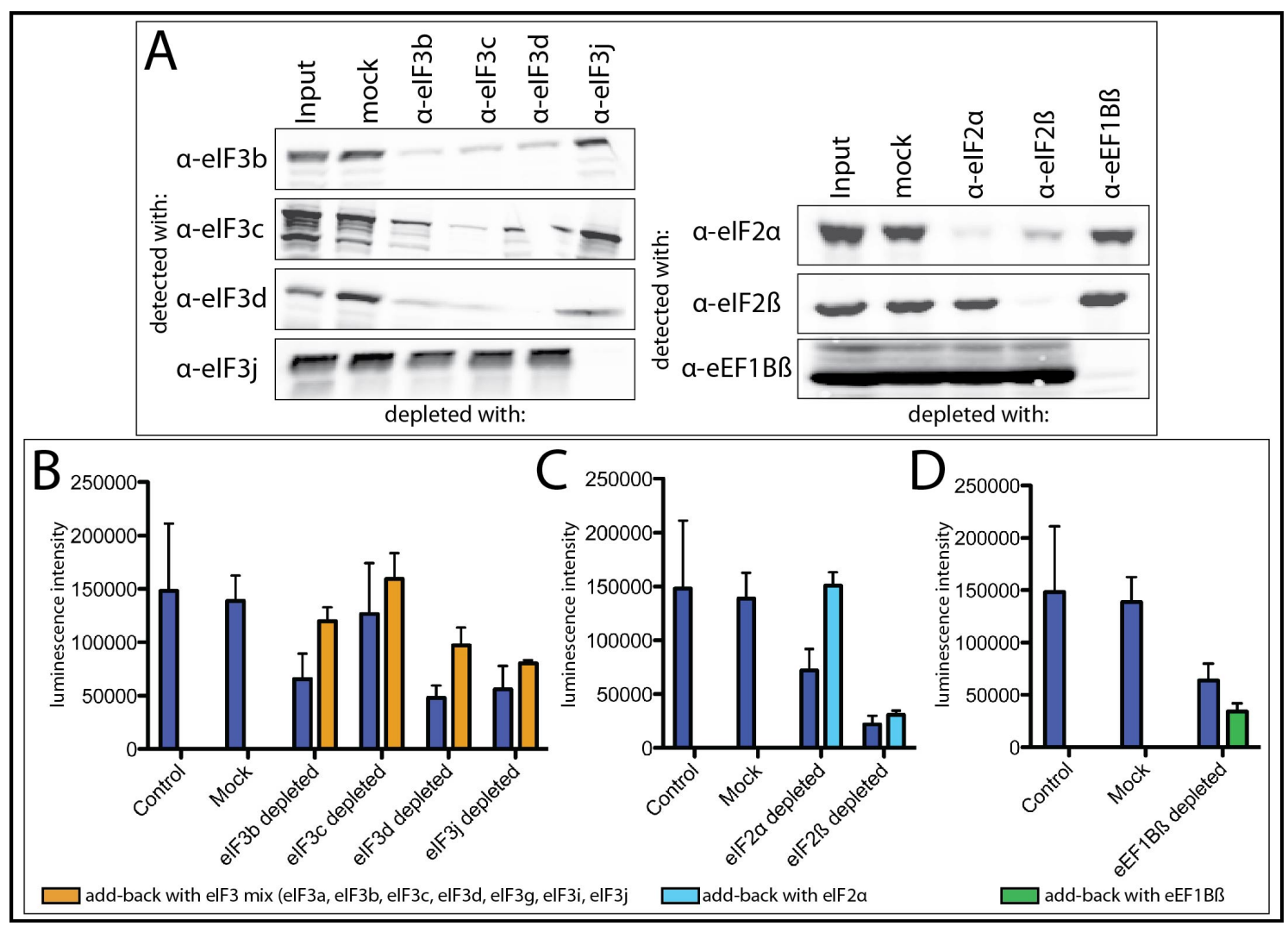

Figure 2.7: Translation efficiency can be partially restored by adding back recombinant factors

Translation initiation factors were depleted from wheat germ extract by subunit specific antibodies. (A): Depleted extracts were analyzed by Western Blot for their target protein content and for possible co-precipitation of additional subunits. As control, a non-depleted extract (Input) and a mock depleted extract (depleted with $\alpha$-GFP antibodies) was analyzed in parallel. Primary antibodies were used at a concentration of $1 \mu \mathrm{g} / \mathrm{ml}$, secondary goat $\alpha$-rabbit IRyde antibodies were used at a concentration of 1:50.000. (B-D): In vitro translation assays were performed with the depleted extracts (blue bars). Further, depleted extracts were supplemented with either single recombinant initiation factors or initiation factor mixes prior to translation (orange: add-back with an eIF3 mix, cyan: add-back with $\mathrm{eIF} 2 \alpha$, green: add-back with $\mathrm{eEF} 1 \mathrm{~B} \beta$ ). As control, non-depleted extract was run in parallel. The translation efficiency was analyzed by estimating the amount of produced Firefly luciferase during the translation reaction by performing a luciferase activity assay using the BioTek Synergy H4 Plate Reader. 
Next, extracts were depleted with antibodies against GFP (mock), eIF3b, eIF3c, eIF3d, eIF $3 j$, eIF $2 \alpha$, eIF $2 \beta$ and eEF1B $\beta$ and analyzed by Western Blot. These extracts were subsequently used in translation assays, supplemented with recombinant translation factors as add-back to restore translation efficiency. The Western Blot analysis in figure 2.7A shows that upon depletion with antibodies against eIF3b, eIF3c and eIF3d, other complex components co-deplete as well. However, the depletion is incomplete as residual amounts of all tested eIF3 subunits can still be detected. Again, eIF3j is an exception such that it does not co-deplete with the other eIF3 subunits but can be efficiently removed from the extract by eIF3j specific antibodies. Depletion with antibodies against eEF1B $\beta$ leads to an almost complete depletion of the endogenous protein in this experiment. Obviously the incomplete depletion seen in figure 2.6 was due to technical errors. Depletion with $\alpha$-eIF $2 \alpha$ results in a drastic reduction of endogenous protein, however, eIF $2 \beta$ levels remain unaffected. Interestingly, depletion of eIF $2 \beta$ does not only lead to an efficient reduction of endogenous target protein but also leads to a decrease in eIF $2 \alpha$ levels.

Figures 2.7B-D show the translation efficiencies of the depleted extracts (dark blue bars) and depleted extracts supplemented with recombinant factors in order to restore translation efficiencies (orange, cyan, green bars). Extracts depleted by $\alpha$-eIF3b, $\alpha$-eIF3d and $\alpha$ eIF3j antibodies perform $\sim 2$-fold less efficient as non-depleted or mock depleted extracts, whereas eIF3c-depleted extracts shows a higher amount of produced Firefly luciferase. Add-back with a mix of recombinant eIF3 subunits (eIF3a, eIF3b, eIF3c, eIF3d, eIF3g, eIF3i, eIF3j) was able to elevate the amount of produced reporter protein in all cases, indicating that the drop in translation efficiency might be truly due to a reduction of eIF3 in the extract. However, comparing the measured translation efficiencies of eIF3 depleted extracts between this experiment and the previously described one (figure 2.6A) reveals a major problem when working with the in vitro wheat germ translation assay. The extract seems to be extremely sensitive towards even slight changes in buffer composition and salt concentrations, so that the reproduction of results is nearly impossible.

Extracts with reduced amounts of $\operatorname{eIF} 2 \alpha$ and $\operatorname{eIF} 2 \beta$ show less efficient translation as compared to the non-treated or mock depleted extracts. Western Blot analysis showed that upon eIF $2 \beta$ depletion, the amount of eIF $2 \alpha$ is reduced as well, explaining the more drastic effect in the eIF2 $\beta$ depleted extracts. Add-back experiments were performed with recombinantly expressed and purified eIF $2 \alpha$. It is able to restore translation efficiency in the eIF $2 \alpha$ depleted samples, but fails to rescue the effect in eIF $2 \beta$ depleted extracts. Here eIF $2 \beta$ is still lacking and thus the eIF2 complex can not function properly in initiator tRNA binding and delivery to the ribosome.

Depletion of eEF1B $\beta$ again greatly reduces translation efficiencies, however adding back $\mathrm{eEF} 1 \mathrm{~B} \beta$ seems to hinder proper protein synthesis even further. A possible explanation 
might be that the eEF1B complex in plants consists of three subunits eEF1B $\alpha, \operatorname{eEF} 1 \mathrm{~B} \beta$ and $\mathrm{eEF} 1 \mathrm{~B} \gamma$. Addition of high concentrations of only one subunit could lead to a competition in binding resulting in heterodimeric complexes failing to form the heterotrimeric complex.

In summary, we could show that the in vitro wheat germ translation assay is a possible tool to analyze limitations and necessities of the translation apparatus, however, the magnitude of technical hurdles makes it - at least at the moment - impossible to obtain clear and trustworthy results. So far, we did not succeed to efficiently deplete the eIF3 complex from the extracts and also depletion of other target proteins resulted in small amounts of remaining endogenous protein. Yet another problem is that the antibodies used most likely only recognize a defined isoform of the protein. Possible other versions of target protein could function in a highly similar manner but cannot be detected by the setup we used. Furthermore, we can not be certain if all recombinant factors we use for add-back experiments are fully functional. In principle, each protein used must be priorly analyzed in depth, considering its folding state, interactions within the wheat germ extract or posttranslational modifications that might be required for proper function but lack when produced in E.coli. Further, the assay itself requires optimization such that obtained data can be reproduced, thereby also determining and abolishing certain limitations in the extract.

Given the multiplicity of parameters to be improved to eventually receive conclusive and convincing data we decided that the project can not be successfully completed in the given time frame and it is hence wisely to change the project.

\subsubsection{Eukaryotic translation initiation factor $5 \mathrm{~A}$ (elF5A) and its effect on poly-proline encoding mRNAs}

eIF5A is a small eukaryotic translation factor with so far unknown function in higher eukaryotes. Its specific interaction with the nuclear export factor Exportin 4 was discovered in our department (Lipowsky et al., 2000), hence raising interest not only for the interaction between the export receptor and its cargo but also for the role of eIF5A during translation.

Recent studies on the bacterial translation factor EF-P, which is homologous to eIF5A, revealed that it is essential for the translation of mRNAs encoding for proline rich proteins. Poly-proline stretches cause the ribosome to pause or even to stall on the mRNA and EF-P is required to resume translation of the transcript (Doerfel et al., 2013). Subsequently, Gutierrez et al. (2013) showed in vivo that eIF5A has a similar function in yeast. Is this also the case in higher eukaryotes? 


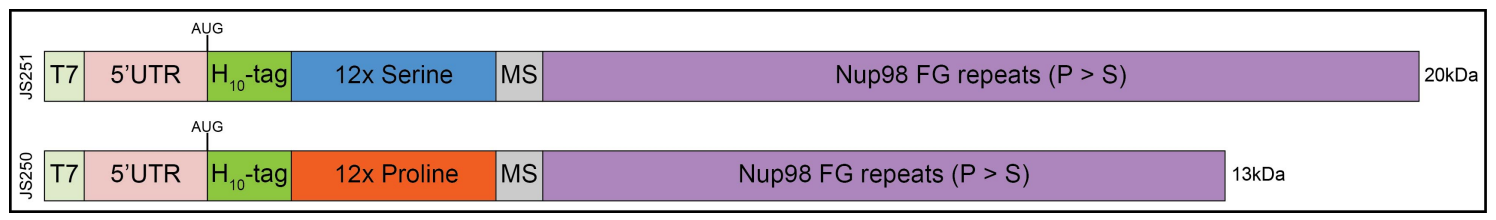

Figure 2.8: Reporter constructs

To monitor the effect of eIF5A on poly-proline encoding mRNAs, reporter constructs were designed and cloned containing either a poly-serine stretch (12xSerine) or a poly-proline stretch (12xProline). A $\mathrm{H}_{10}$-tag was fused $\mathrm{N}$ terminally to provide a comparable translation start in both constructs. To facilitate proper ${ }^{35} \mathrm{~S}-\mathrm{Met}$ incorporation, a short methionine/serine rich stretch (MS) was introduced, followed by parts of the Nup98FG repeat domain in two different length. Prior to that, all prolines in the Nup98FG repeat domain were exchanged to serines $(\mathrm{P} \rightarrow \mathrm{S}$, performed by Dr. Aksana Labokha).

To address this question, we used the in vitro rabbit reticulocyte lysate translation system. Simultaneous translation of poly-serine and poly-proline reporter constructs in extracts depleted of eIF5A by specific antibodies should reveal the necessity of the translation factor during the synthesis of proline rich reporters. The antibodies used were previously purified and well characterized, recognizing two different epitops on human eIF5A (described in Lipowsky et al., 2000). These were positively tested for cross-reactivity with rabbit eIF5A (data not shown).

The new poly-serine or poly-proline reporter constructs were designed as follows (from N-terminus to C-terminus): (i) a $\mathrm{H}_{10}$-tag (ii) a stretch of either 12 consecutive serines or 12 consecutive prolines (iii) a methionine rich stretch for proper labeling with ${ }^{35} \mathrm{~S}-\mathrm{Met}$ and (iv) parts of the Nup98-FG domain in which all prolines had been mutated to serines (performed by Dr. A. Labokha). The construct containing the serine stretch is fused to a longer part of the FG-domain, the poly-proline construct is slightly shorter (see figure 2.8). This enables to distinguish the two constructs after simultaneous translation.

In order to deplete eIF5A from the extract, the rabbit reticulocyte lysate was incubated with ProteinA Sepharose to which antibodies against an N-terminal human eIF5A epitope had been covalently attached. As control, the lysate was also incubated with ProteinA Sepharose (empty beads (e.b.) control) and $\alpha$-GFP coupled Sepharose (mock depletion). The depletion efficiency was analyzed by Western Blot (figure 2.9A), showing no change in endogenous protein amount when comparing the input with the empty bead control or the mock depleted extract. Depletion of endogenous eIF5A by $\alpha$-eIF5A specific antibodies was highly efficient. No endogenous protein was detected when incubating $200 \mu \mathrm{l}$ or $100 \mu \mathrm{l}$ lysate with $50 \mu$ l ProteinA-IgG beads, minor amounts of protein were observed when $500 \mu \mathrm{l}$ extract had been used, thereby exceeding the capacity of the beads.

Next, the rabbit reticulocyte in vitro translation assay was performed, simultaneously using the long poly-serine and short poly-proline reporter constructs. Untreated lysate (Input), lysate thawn once (Input thawn), and lysates depleted via empty beads, $\alpha$-GFP and $\alpha$-eIF5A were compared. In addition, the $\alpha$-eIF5A depleted extract was supplemented with $5 \mu \mathrm{M}$ natively purified human eIF5A (see figure 2.9B). Interestingly, the two reporter 
constructs were translated under all tested conditions. A decrease in translation efficiency of the poly-proline construct in eIF5A depleted lysates could not be observed. Note, that the lower signal intensity for the poly-proline constructs compared to the poly-serine constructs was observed in all experiments.

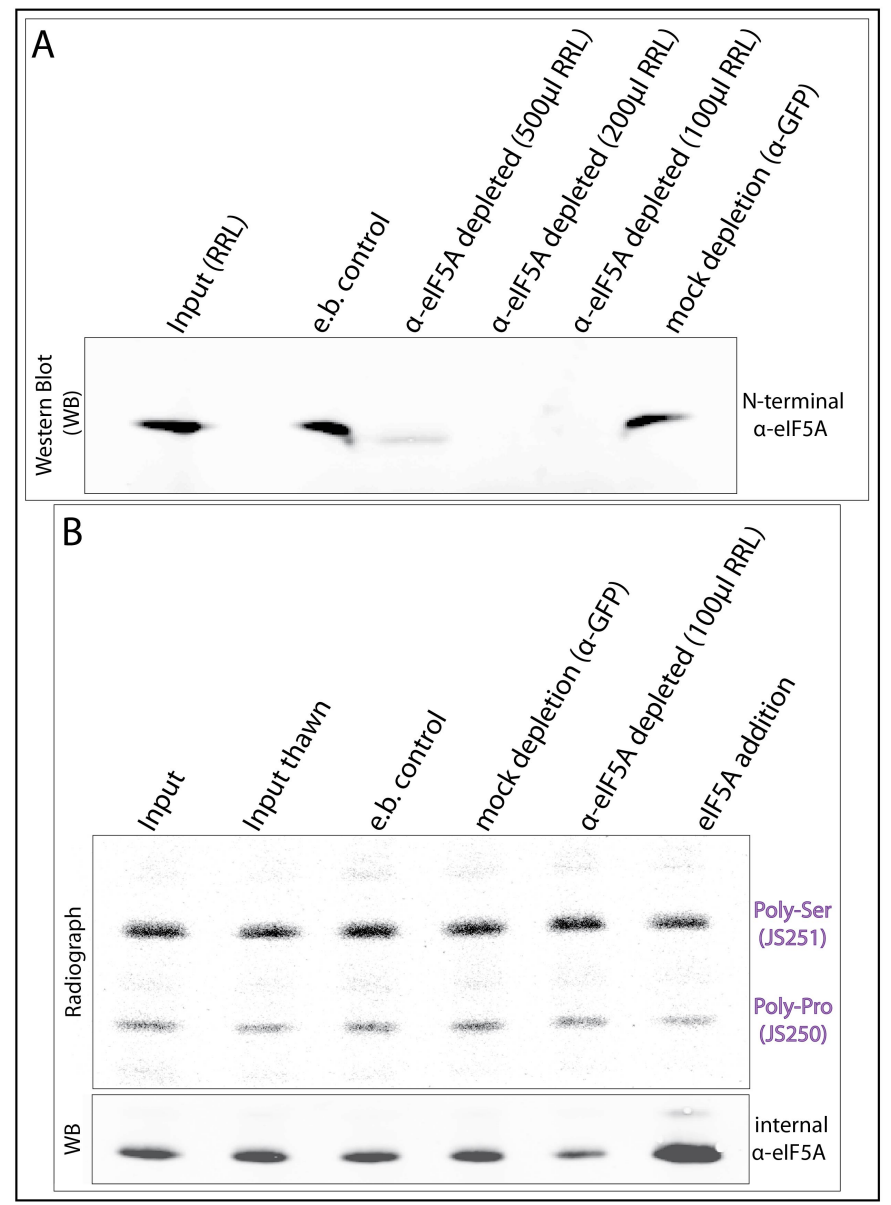

Figure 2.9: elF5A depletion does not affect translation of a poly-proline reporter

(A): $100 \mu \mathrm{l}, 200 \mu \mathrm{l}$ and $500 \mu \mathrm{l}$ Rabbit Reticulocyte Lysate (RRL) were depleted with monoclonal $\alpha$-hs eIF5A antibodies raised against an N-terminal epitope of human eIF5A. The antibody was coupled to ProteinA Sepharose in a concentration of $3 \mu \mathrm{g} / \mu \mathrm{l} ; 50 \mu \mathrm{l}$ beads were used for depletion. The remaining amount of endogenous protein in the extracts was analyzed by Western Blot. As control, non-depleted and mock depleted ( $\alpha$-GFP antibody coupled to ProteinA Sepharose) extracts were analyzed as well. (B): In vitro translation assays were performed using the empty beads-, mock- and $\alpha$-eIF5A - depleted extracts, including control samples of non-treated extracts (Input) and extracts thawn once (Input thawn). In addition, $\alpha$-eIF5A depleted extracts were supplemented with $5 \mu$ l natively purified human eIF5A (eIF5A addition). In all extracts tested, a poly-serine (Poly-Ser) and a poly-proline (Poly-Pro) reporter construct were translated simultaneously as described in 4.2.6.3. Ammonium sulfate precipitated samples were loaded on a gradient polyacrylamid gel and subsequently applied to a radiographic film. The film was scanned after over night exposure using the Fujifilm FLA7000 scanner (Radiograph). In addition, a second Western Blot was performed with an $\alpha$-hs eIF5A antibodies recognizing a epitope located on a linker region between two domains (WB). All primary antibodies were used at a concentration of $1 \mu \mathrm{g} / \mathrm{ml}$, secondary antibodies were used in a 1:50.000 ratio.

As control, the extracts were once more analyzed by Western Blot, this time using a human $\alpha$-eIF5A antibody recognizing not the N-terminal amino acid stretch but an internal epitope located in the linker region between two domains of eIF5A (figure 2.9B, WB, lower panel). This approach revealed that endogenous eIF5A protein is still present 
in $\alpha$-eIF5A depleted sample. Most likely, the two different human $\alpha$-eIF5A antibodies recognize slightly different pools of eIF5A in the reticulocyte lysate. By using only the monoclonal antibody recognizing the N-terminal eIF5A epitope for depletion, a residual pool of eIF5A remained in the lysate, possibly enough to ensure a proper and efficient translation of the poly-proline containing reporter.

In subsequent experiments, the lysate was depleted with both $\alpha$-eIF5A antibodies in consecutive rounds, however, a complete depletion of the endogenous protein from the lysate could not be reached. The depleted lysates were also not able to impair the synthesis of the proline rich reporter.

The following section will describe an alternative approach, in which endogenous eIF5A is not depleted but sequestered.

\subsection{Can nuclear transport factors act as compartment specific inhibitors of translation?}

Despite the theory of nuclear translation, a spatial-temporal separation of transcription happening in the nucleus and translation acting in the cytoplasm is highly beneficial for eukaryotic cells. If these two processes were directly coupled, the probability of translating non- or mis-spliced mRNA molecules to proteins would be high, resulting in proteins that are mislocalized and might even have dominant negative effects. Eukaryotic cells governed a multitude of mechanisms to prevent an intermixing of transcription and translation, such as keeping the level of translation factors in the nucleus actively low. Nuclear transport factors, mainly nuclear export factors (exportins), can shuttle out proteins from the nucleus to the cytoplasm in a RanGTP dependent manner (see section 1.2.1). Exportin 4, respectively, is known to interact with the small translation factor eIF5A and to efficiently remove it from the nuclear interior (Lipowsky et al., 2000).

As already mentioned in the previous section, we aim to analyze the role of eIF5A in the translation of proline rich proteins in higher eukaryotes. eIF5A should therefore be depleted from the translation extract by specific antibodies and the ability of the depleted extract to translate a poly-proline continuing reporter constructs should be analyzed. So far depletion of endogenous eIF5A from the extract was incomplete. An alternative approach to analyze the role of eIF5A during translation exploits the feature of Exportin 4 to specifically interact with the translation factor. By supplementing translation assays with the nuclear export factor endogenous eIF5A can be sequestered and thus hindered to act during translation.

Interestingly, Exportin 4 is not the only nuclear transport factor binding translation factors and shuttling them out of the nucleus. Bohnsack et al. (2002) showed interactions 
between e.g. Importin 13 and eIF1A as well as a tRNA mediated interaction between Exportin 5 and eEF1A. Recent data in the lab further showed that pull down assays with the nuclear export factor Crm1 were able to enrich most translation (initiation) factors on the column in a Ran dependent manner (K. Kirli, unpublished data). Thus, the question arises if nuclear transport factors are not only able to bind translation factors in a direct or indirect manner but if this binding is also able to sequester translation factors in a way that the translation efficiency of a supplemented in vitro translation assay decreases. Even further, it can be hypothesized that transport factors act as compartment specific inhibitors of translation, which clearly argues against the idea of nuclear translation.

\subsubsection{Addition of Exportin 4 to an in vitro translation system can reduce the translation efficiency of a poly-proline reporter}

In previous experiments, a poly-serine construct with a longer version of the Nup98FG domain and a poly-proline construct with a shorter version of the Nup98FG domain were used $(\mathrm{P} \rightarrow \mathrm{S}$ mutations in the Nup98FG domains, see figure 2.8). However, the translation efficiencies of these constructs were rather low, leading us to change the $\mathrm{N}$-terminal $\mathrm{H}_{10^{-}}$ tag to a 3xFLAG tag (see figure 2.10, green). When comparing the translation efficiencies of the different constructs, the 3x-FLAG constructs gave a better signal on the radiograph and thus these constructs were used for all following experiments (data not shown). In addition, the Firefly luciferase and the two yeast proteins Eap1 and Ldb17 were fused to a $3 \mathrm{x}-\mathrm{FLAG}$ tag and were used as reporters as well. Eap1 and Ldb17 were chosen due to findings by Gutierrez et al. (2013) that synthesis of these proteins is strongly impaired if eIF5A is non-functional in yeast. The length of the reporter constructs and the amounts of prolines per construct are presented in figure 2.10.

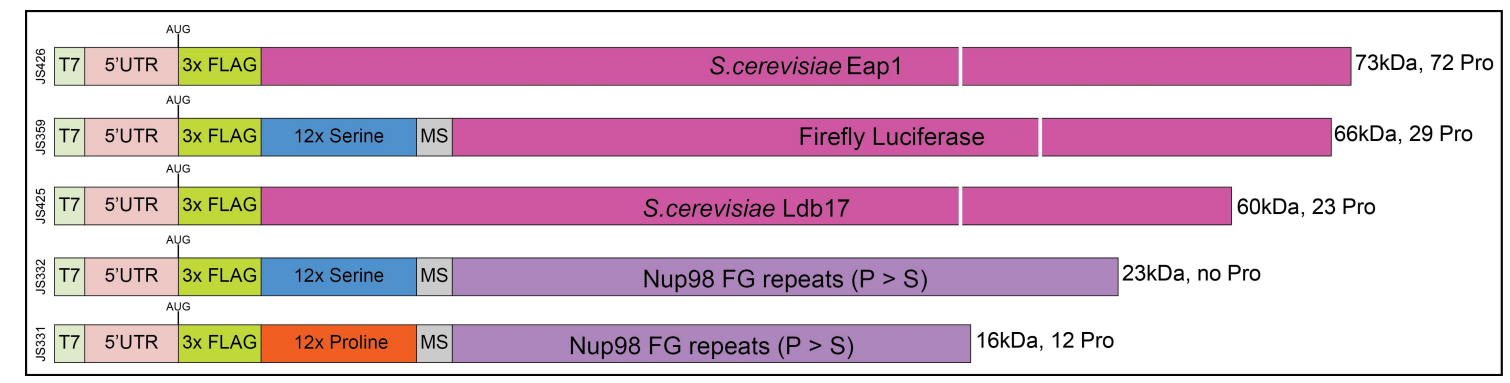

Figure 2.10: In vitro translation reporter constructs with N-terminal 3xFLAG tag

To increase the expression levels of the reporter constructs used in the RRL in vitro translation assay, the N-terminal tag was switched from $\mathrm{H}_{10}$-tag (see figure 2.8) to 3xFLAG tag (green). In addition to the poly-serine and polyproline constructs with Nup98FG domains in different length (violet), additional reporters were constructed: Firefly Luciferase and the two yeast proteins Eap1 and Ldp 17 (pink). The size of the reporter constructs as well as the amount of prolines are listed for each construct.

Figure 2.11 shows the radiograph of the in vitro translation assay using the reporter constructs encoding Firefly Luciferase, Ldb17 and Eap1. Each construct was translated 
in non supplemented reticulocyte lysate, in lysate supplemented with $5 \mu \mathrm{M}$ mmExportin 4 (Xpo4) and lysate supplemented with $5 \mu \mathrm{M}$ mmExportin 4 and $15 \mu \mathrm{M}$ RanQ69L 5 -180 (Exportin 4 and Ran were recombinantly expressed in E.coli and subsequently purified by affinity chromatography and protease elution). All three constructs can be efficiently synthesized in the reticulocyte translation system. When supplementing the lysate with Exportin 4 only, the amount of produced protein does not vary much in comparison to non supplemented lysate in case of Firefly Luciferase and Ldb17. However, full length Eap1 can not be observed any longer. Instead, two lower molecular bands appear, which possibly represent partially translated Eap1 protein caused by ribosome stalling. If the lysate is supplemented with Exportin 4 and Ran, the amounts of produced Firefly luciferase and Ldb17 drop significantly, no translation product for Eap1 can be observed any longer. This effect can not be explained by RanQ69L 5 -180 addition itself. It was shown in independent experiments that Ran does not effect the translation system (see also figure 2.13A).

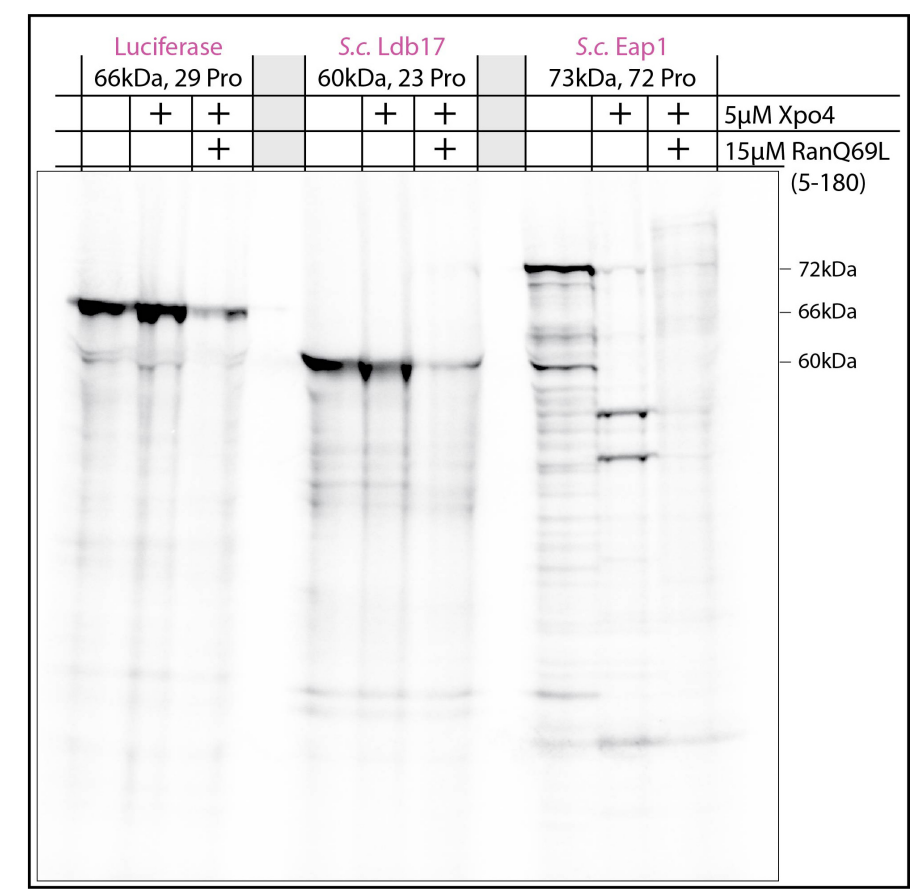

Figure 2.11: Addition of Exportin 4 decreases translation efficiency of proline containing reporters in a Ran dependent manner

Firefly Luciferase and the yeast proteins Ldb17 and Eap1 were translated in the RRL in vitro translation system. The proteins were either N-terminally tagged with 3x-FLAG (Ldb17 and Eap1) or 3x-FLAG-12xSer (Firefly Luciferase). The translation was performed in untreated extracts (first lane), extracts supplemented with $5 \mu \mathrm{M}$ recombinantly purified mmExportin 4 (middle lane) and extracts supplemented with $5 \mu \mathrm{M}$ recombinantly purified mmExportin 4 and $15 \mu \mathrm{M}$ recombinantly purified RanQ69L ${ }_{(5-180)}$ (right lane). The translation reactions were precipitated with ammonium sulfate, the pellet was resuspended in SDS loading buffer and the samples were loaded on a gradient polyacrylamide gel. The gel was subsequently fixed, dried and placed over night on a radiographic film. The film was scanned using the Fujifilm FLA7000 scanner.

Translation of Eap1, which contains 72 proline residues, is already strongly impaired by addition of Exportin 4 only. Possibly, the nuclear transport factor binds eIF5A to some extend even in the absence of Ran, resulting in a decreased pool of fully active eIF5A in the 
extract. This might still be enough to overcome pausing events on mRNA molecules caused by small patches of proline codons, but might be insufficient to release stalled ribosomes. Further adding Ran might stabilize and increase the interaction between Exportin 4 and eIF5A, thereby efficiently sequestering the translation factor.

These data suggest a role of eIF5A in the translation of poly-proline containing proteins also in higher eukaryotes. However, two major experiments are still required to finalize our results. First, the direct interaction of the recombinantly expressed and purified Exportin 4 and Ran and the endogenous rabbit eIF5A needs to be shown. So far preliminary data indicate a direct binding of Exportin 4 and eIF5A in a Ran dependent manner. Possible weak and transient interactions between Exportin 4 and eIF5A in the absence of Ran could not be proven yet. Second, add-back experiments with eIF5A need to be performed. Purification of native eIF5A from HeLa cell lysate is a very time consuming, multistep process yielding in small amounts of native protein. However, the unusual modification on eIF5A, a hypusination, is required for proper function and also for efficient binding of Exportin 4 (Lipowsky et al., 2000; Doerfel et al., 2013; Gutierrez et al., 2013). This modification can not be introduced in vivo by E.coli. Metin Aksu from our lab recently established an efficient technique to introduce the hypusination to recombinantly expressed and purified eIF5A in vitro. Titration of functional eIF5A to the reticulocyte lysate supplemented with Exportin 4 and Ran could restore the translation efficiency of reporter constructs upon a certain concentration threshold, thereby strongly suggesting that the decrease in translation efficiency was caused by sequestration of eIF5A.

\subsubsection{Addition of Crm1 to an in vitro translation system greatly reduces its translation efficiency}

Nuclear transport factors can either act on a small set of proteins, such as CAS binding Importin $\alpha$ or Exportin 6 acting on actin:profiling complexes or they can recognize a multitude of different cargoes via short sequence stretches known as nuclear localization signal (NLS) or nuclear export signal (NES) (Görlich et al., 1994; Wen et al., 1995; Kutay et al., 1997; Stüven et al., 2003). The nuclear export factor Crm1 binds leucin-rich NES containing cargoes in a Ran dependent manner and efficiently removes them from the nuclear interior. Thereby the NES peptide binds to the hydrophobic cleft of Crm1 (Güttler et al., 2010). Recent experiments in our lab showed that Crm1 is able to pull out the majority of translation factors from HeLa cell lysate in a Ran dependent manner (K. Kirli, unpublished data).

To experimentally validate the hypothesis that nuclear transport factors recognizing translation factors are also able to suppress translation, the in vitro reticulocyte translation assay was supplemented with different recombinantly expressed and purified nuclear trans- 
port factors in the absence or presence of Ran (figure 2.12). As control, a $5 \mu \mathrm{M}$ nuclear transport factors solution was for 10 minutes to denature the protein and subsequently centrifuged. The remaining supernatant was used as buffer control in all following experiments (buffer control). This approach was used to create a control situation in which transport factors are not present, however, possible co-purified substances might remain in solution and effects of these on overall translation can be ruled out. Even further this supernatant has the exact same buffer composition as the protein solution used for supplementing the lysate.

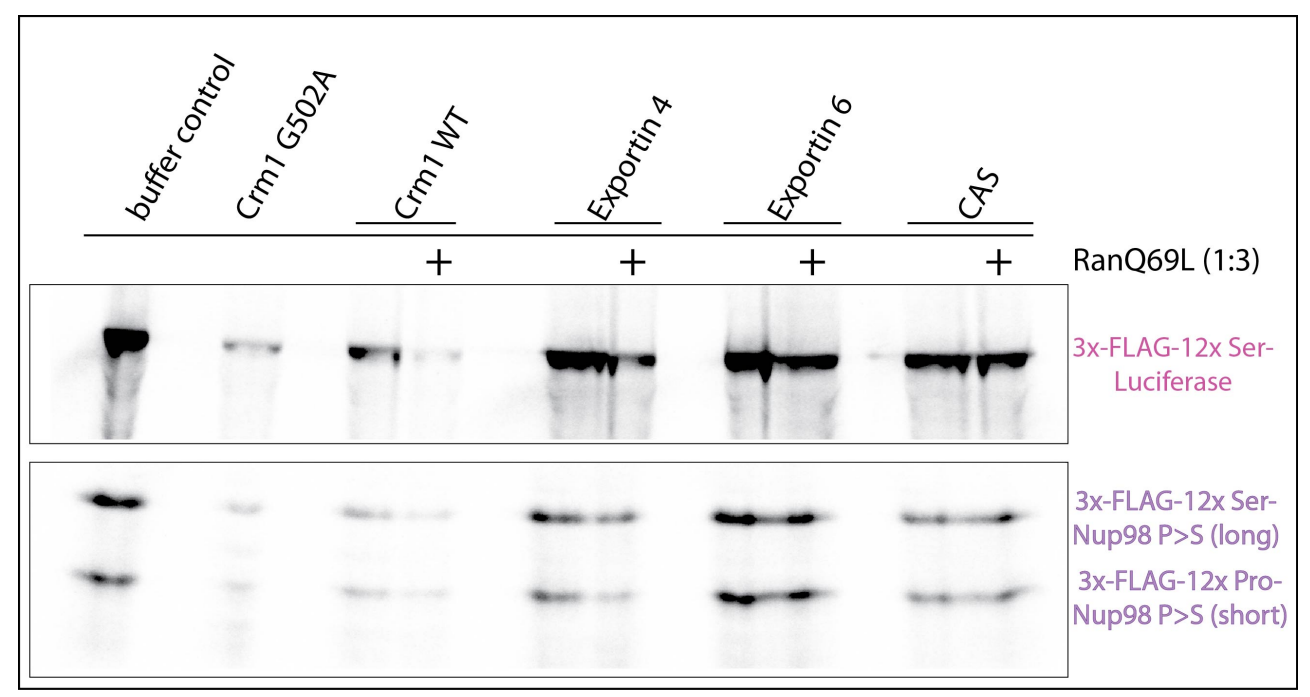

Figure 2.12: Nuclear export factor $\mathrm{Crm} 1$ decreases overall translation rates in an in vitro translation assay in a Ran dependent manner

Reporter constructs coding for Firefly Luciferase or Nup98FG repeat domain $(\mathrm{P} \rightarrow \mathrm{S})$ fused to either $12 \mathrm{xProline}$ or $12 x$ Serine were expressed in the RRL in vitro translation system (all constructs N-terminally tagged with $3 x-F L A G$ tag). The RRL extract was either non supplemented (buffer control) or supplemented with $5 \mu \mathrm{M}$ nuclear export factors $\left(m m C r m 1_{\mathrm{G} 502 \mathrm{~A}}\right.$ (Ran independent Crm1 mutant), mmCrm1 WT, mmExportin 4, hsExportin6 or hsCAS). To observe a possible Ran dependency the extracts were further supplemented with $5 \mu \mathrm{M}$ nuclear export factors and $15 \mu \mathrm{M}$ with RanQ69L (5-180) (marked by + ). All nuclear transport factors and Ran were recombinantly expressed and purified. The translation reactions were precipitated with ammonium sulphate, the pellet was resuspended in SDS loading buffer and the samples were loaded on a gradient polyacrylamide gel. The gel was subsequently fixed, dried and placed over night on a radiographic film. The film was scanned using the Fujifilm FLA7000 scanner.

The effects of the nuclear export factors Crm1 (as WT and as Ran independent G502A mutant), Exportin 4, Exportin 6 and CAS on the translation of the firefly luciferase reporter construct and on the poly-serine/poly-proline constructs were tested in absence and presence of Ran (except $\operatorname{Crm} 1_{\mathrm{G} 502 \mathrm{~A}}$, which does not require Ran). When supplementing the lysate with buffer only (buffer control, figure 2.12) efficient translation of all three reporter constructs can be observed. Addition of Crm1 $1_{\mathrm{G} 502 \mathrm{~A}}$ leads to a strong decrease in signal intensity implying a lower translation efficiency. When adding Crm1 WT, the effect is milder, but by further adding RanQ69L $\mathrm{L}_{(5-180)}$, the translation efficiency of all three reporter constructs drops significantly. Addition of Exportin 4 alone does not affect translation rates, however, adding Exportin 4 and RanQ69L $\mathrm{L}_{(5-180)}$, leads to a decrease in translation efficiency for the luciferase and the poly-proline construct whereas 
the poly-serine constructs remains unaffected. Exportin6, the nuclear export factor for actin:profiling complexes, or CAS, required for shuttling Importin $\alpha$ to the cytoplasm, do not affect the three tested reporter constructs either in presence or absence of Ran.

The data suggests that nuclear transport factors involved in the export of translation factors from the nuclear interior have an ability to (partially) suppress translation when present in an in vitro translation system, whereas other nuclear transport factors that are highly similar in shape and size but have no function in nuclear export of translation factors do not effect translation. Further, the observed decrease in translation efficiencies seems to be Ran dependent.

\subsubsection{Decrease in translation can not be rescued by blocking or mutating the hydrophobic cleft of Crm1}

Crm1 addition leads to a dramatic decrease in translation efficiency of various reporter constructs (see section 2.3.2). By adding different concentration of Crm1 and Ran (1:1.5 ratio) to the lysate, we were able to observe that the drop in translation efficiency seems to be concentration dependent (figure 2.13A). Compared to the buffer control, addition of $1 \mu \mathrm{M}$ WT Crm1 even in presence of Ran does not affect synthesis of the reporter constructs (here the poly-serine and poly-proline Nup98FG domain constructs), whereas addition of $5 \mu \mathrm{M}$ WT Crm1 or $10 \mu \mathrm{M}$ Crm1 results in significantly less translated reporter protein. Interestingly, samples treated with Crm1 showed a similar effect as compared to addition of Crm1 and Ran. Note that the previously described experiment was performed using Ran in 3-fold excess over the nuclear transport receptor, here the excess was only 1.5-fold. Figure $2.13 \mathrm{~A}$ also shows that addition or Ran does not negatively affects translation rates even at elevated concentrations.

Previous experiments showed that addition of Crm1 to an in vitro translation assay decreases overall translation rates. Yet, it remains unclear by which mechanism this effect occurs. Most likely, Crm1 is able to bind certain translation factors via NES stretches, thereby not only capturing these subunits but also the attached complexes. Crm1 thus might act as platform to which more and more factors attach, thereby being hindered to function in the translation process. If this assumption holds true, the effect should be reversible by preventing binding of the NES to the hydrophobic cleft of Crm1.

One possibility to block the hydrophobic cleft of Crm1 is to additionally supplement the translation lysate with a strong binder of Crm1, thereby possibly competing out all other interactions between Crm1 and its cargoes in the lysate. Thus, the RRL was supplemented with increasing concentrations of the mmCrm1 $1_{\mathrm{G} 502 \mathrm{~A}}$ mutant $(1 \mu \mathrm{M}, 5 \mu \mathrm{M}$, or $10 \mu \mathrm{M})$ or $\mathrm{mmCrm} 1_{\mathrm{G} 502 \mathrm{~A}}$ and $\mathrm{NS} 2$ peptide in 5 -fold excess over the nuclear transport factor. The peptide is the NES sequence found in the NS2 protein of parvovirus Minute Virus of Mice 
(MVM) and was shown to bind Crm1 with higher affinity as regular NESs (Engelsma et al., 2008). Figure 2.13B shows that upon addition of low concentrations of Crm1 $(1 \mu \mathrm{M})$ and NS2 peptide $(5 \mu \mathrm{M})$, no changes in translation efficiency compared to the buffer control can be observed. Upon addition of higher Crm1 concentrations, the reporter constructs are significantly less efficiently translated. However, addition of the NS2 peptide is not able to reverse the effect, it even seems to further weaken protein synthesis. Note that Crm1 and the NS2 peptide were separately added to the lysate and not as a preformed complex. Hence, receptor and cargo first need to find each other in the crowded lysate milieu. It also remains unclear if the peptide is harmed by proteases present in the extract.

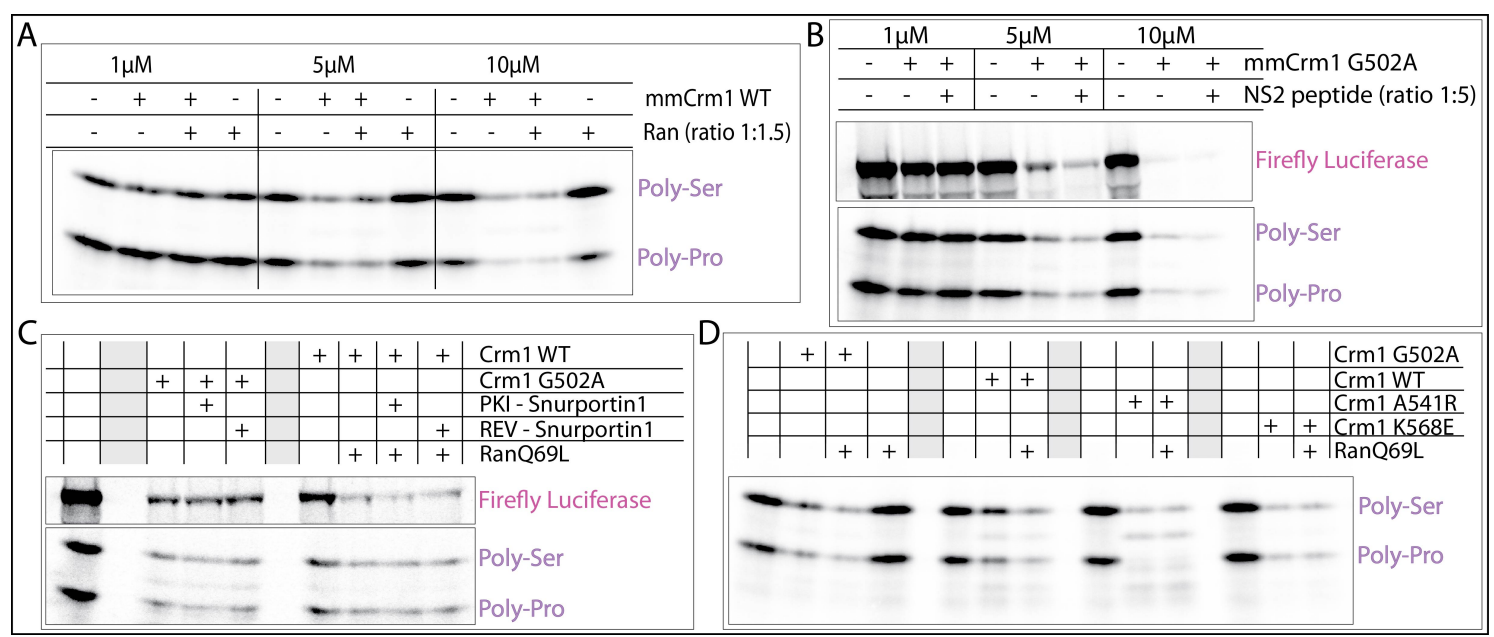

Figure 2.13: Decrease in translation upon Crm1 addition can not be reversed by blocking or mutating Crm1s hydrophobic cleft

The following reporter constructs were used in these experiments: 3xFLAG-12xSer-Firefly Luciferase (Firefly Luciferase), 3xFLAG-12xSer-Nup98 FG domain $\mathrm{P} \rightarrow \mathrm{S}_{(\text {long) }}$ (Poly-Ser), 3xFLAG-12xPro-Nup98 FG domain P $\rightarrow \mathrm{S}$ (short) (Poly-Ser)(for details see figure 2.10). (A): Poly-Ser and Poly-Pro reporter constructs were translated in RRL extracts supplemented with buffer only, $1 \mu \mathrm{M}, 5 \mu \mathrm{M}$, or $10 \mu \mathrm{M}$ mmCrm1 WT, or mmCrm1 WT and RanQ69L $(5-180)$ in a 1:1.5 ratio. (B): Firefly Luciferase and the Poly-Ser and Poly-Pro reporters were translated in RRL extracts supplemented with $0 \mu \mathrm{M}, 1 \mu \mathrm{M}, 5 \mu \mathrm{M}$, or $10 \mu \mathrm{M} \mathrm{mmCrm} 1_{\mathrm{G} 502 \mathrm{~A}}$ in presence or absence of NS2 peptide, which is known to strongly bind Crm1 in vitro. The peptide was added in 5-fold excess over the transport factor. (C): Firefly Luciferase and the Poly-Ser and Poly-Pro reporters were translated in RRL extracts supplemented with $5 \mu \mathrm{M}$ mmCrm1 WT or mmCrm1 $1_{\mathrm{G} 502 \mathrm{~A}}$ in combination with RanQ69L $\mathrm{L}_{(5-180)}$ and the natural Crm1 cargo Snurportin1 fused to the PKI- or REV-type nuclear export signals. RanQ69L $(5-180)$ was added at a concentration of $15 \mu \mathrm{M}$, the snurportin fusion proteins at a concentration of $10 \mu \mathrm{M}$. (D): Poly-Ser and Poly-Pro reporter constructs were expressed in RRL extracts supplemented with various mmCrm1 versions at a concentration of $5 \mu \mathrm{M}$. In addition, RanQ69L $(5-180)$ was added in 3 -fold excess. Crm1 $1_{\mathrm{G} 502 \mathrm{~A}}$ is a Ran independent Crm1 mutant, $\mathrm{Crm} 1_{\mathrm{A} 541 \mathrm{R}}$ and Crm1 $1_{\mathrm{K} 568 \mathrm{E}}$ are mutants known to block the hydrophobic cleft thereby preventing binding of known strong Crm1 binders.

All mmCrm1 versions, the peptides and Snurportin constructs were recombinantly expressed in E.coli and purified by affinity chromatography. All translation reactions were precipitated with ammonium sulphate, the pellet was resuspended in SDS loading buffer and the samples were loaded on a gradient polyacrylamide gel. The gel was subsequently fixed, dried and placed over night on a radiographic film. The film was scanned using the Fujifilm FLA7000 scanner.

Another approach to block the hydrophobic cleft is the addition of Crm1s natural cargo Snurportin1 fused to the nuclear export signals PKI or REV for even stronger interaction between transport factor and cargo. In addition to blocking the hydrophobic cleft, a substantial amount of Crm1s surface will be additionally shielded by Snurportin 1 (Mönecke et al., 2009). In the following experiment, shown in figure $2.13 \mathrm{C}$, the RRL was supple- 
mented with either $5 \mu \mathrm{M}$ Crm1 WT or $\operatorname{Crm} 1_{\mathrm{G} 502 \mathrm{~A}}(5 \mu \mathrm{M})$ with and without Snurportin1 $(10 \mu \mathrm{M})$ either fused to PKI-NES or REV-NES. When using mmCrm1 WT, RanQ69L $\mathrm{L}_{(5-180)}$ in 3-fold excess was added as well. All three tested reporter constructs could be expressed in the buffer control, whereas addition of $m m C r m 1_{\mathrm{G} 502 \mathrm{~A}}$ and $\mathrm{mmCrm} 1 \mathrm{WT}$ and Ran to the extracts strongly impaired protein synthesis. Addition of Crm1 WT only showed a milder decrease in translation efficiencies, once again showing the Ran dependency of the observed effect. However, addition of the Snurportin1 fusion proteins was not able to restore translation efficiency. In this experiment, receptor and cargo were also added separately to the lysate. In future experiments, the complexes need to be preformed, to be certain that Crm1 is indeed blocked by its cargo.

Finally, instead of blocking the hydrophobic cleft by nuclear export signals, the Crm1 hydrophobic cleft itself was modified. Dr. Hema Chug showed that by mutating certain residues in the Crm1 hydrophobic cleft, binding of PKI can be completely abolished (unpublished data). Mutants $m m C r m 1_{\mathrm{A} 541 \mathrm{R}}$ and $\mathrm{mmCrm} 1_{\mathrm{K} 568 \mathrm{E}}$ were analyzed in the context of the in vitro translation assay in comparison to $\mathrm{mmCrm} 1 \mathrm{WT}$ and $\mathrm{mmCrm} 1_{\mathrm{G} 502 \mathrm{~A}}$ in presence and absence of Ran as shown in figure 2.13D. Upon addition of Crm1 WT and Crm $1_{\mathrm{G} 502 \mathrm{~A}}$, translation efficiency of the poly-serine and poly-proline reporter constructs are impaired. Surprisingly, this is also the case for the two hydrophobic cleft mutants $m m C r m 1_{\mathrm{A} 541 \mathrm{R}}$ and $\mathrm{mmCrm} 1_{\mathrm{K} 568 \mathrm{E}}$. Two possible explanations are: first, the mutations in the hydrophobic cleft were analyzed and screened for preventing binding of the PKI NES peptide. However, natural occurring NES signals can have a great variety of amino acid compositions and might bind even the mutated Crm1 versions. This theory needs to be experimentally validated by pull out experiments with the Crm1 mutants. Second, binding of translation factors to Crm1 might not only occur via the hydrophobic cleft but also via interactions with the hydrophobic patches on the Crm1 surface. However, this binding mode would not explain the Ran dependency showed for Crm1 WT.

In conclusion, the nuclear export factor Crm1 can (partially) impair protein synthesis in a Ran dependent manner when added to an in vitro translation assay. Combined with the findings that almost all translation factors can be found in the broad cargo range of the NTR, sequestration most likely occurs via a direct or indirect interaction between Crm1 and the translation factors. However, blocking or mutating the hydrophobic cleft of Crm1 and thereby preventing cargo binding, does not lead to a rescue of the translation efficiency of the extract. Hence, the mode of translation sequestration by Crm1 remains unclear and needs to be further analyzed. 


\subsection{A activated resin as novel tool for affinity chromatography}

Affinity chromatography is a crucial technique in biochemistry. It allows purification of proteins or protein complexes of interest from heterogenous protein mixtures, e.g. a cell lysate, but it also allows to study binding interactions to a particular protein or substance. The technique is based on the interaction between two molecules. These can be two proteins, e.g. an antibody and its corresponding antigen, but also receptor-ligand pairs as it is the case for streptavidin and biotin. For affinity chromatography, one of the interaction partners is immobilized on a stationary phase (Sepharose, silica or magnetic beads) whereas the other partner either is the protein of interest itself or additionally attached to the protein of interest. Attachment occurs either by creating fusion constructs or by chemical modification. When applying a protein mixture to the stationary phase, the affinity tag-containing protein of interest can specifically bind to the resin, whereas other proteins cannot. Many proteins, such as streptavidin or ProteinA can be immobilized to the stationary phase. These systems are commercially available and are well established tools. Other proteins, such as nanobodies or affibodies can be used for immobilization as well. Nonetheless, a toolbox allowing to easily create tailor-made affinity resins specifically for a desired target protein and the corresponding experiment, would be highly beneficial for many applications in protein biochemistry.

A

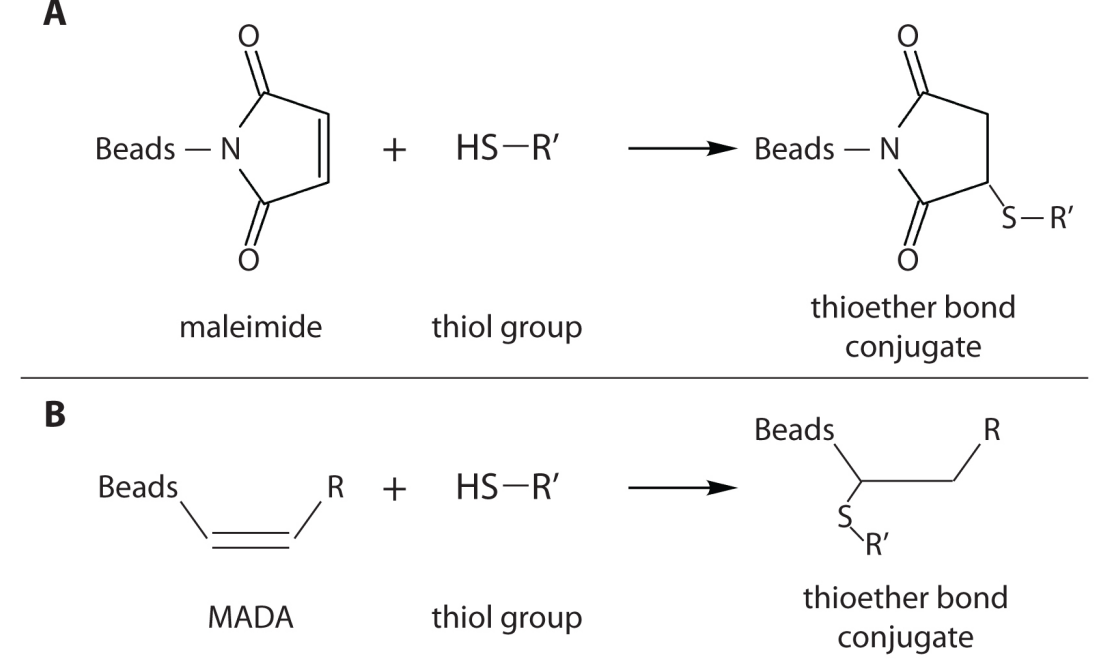

Figure 2.14: Coupling chemistry using maleimide and MADA

Peptides or proteins can be immobilized to a solid material by functionally activating the phase with maleimide derivatives forming a stable thioester conjugate upon reaction with thiol groups on the peptide. A shows the reaction occurring when using maleimide, $\mathbf{B}$ shows the reaction utilizing MADA.

Here, a MADA activated resin is presented, to which proteins containing reduced cysteins can be covalently attached. The chemistry can be applied to Sepharose, silica and magnetic beads and thus further broadens the field of applications.

Immobilization of a ligand to a solid phase requires the activation of the phase with a com- 
pound reactive towards a functional group present on the ligand, most commonly used are primary amines or thiol groups. Thiol groups can be found in the side chains of cysteins and coupling is mainly performed by utilizing haloacetamide derivatives. Maleimids and maleimid derivatives can be alternatively used for immobilization of ligands via thiol groups (see figure 2.14, A and B).

We use here matrices into which MADA groups had been introduced by solid-phase chemical synthesis, starting either from amino-substituted Sepharose or aminopropyl trimethoxy silane-modified silica particles (prepared by Prof. Dirk Görlich).

\subsubsection{MADA Matrix is highly specific for reduced cysteins}

The created MADA 2B Sepharose was analyzed for its coupling specificity towards proteins with reduced cysteins (figure 2.15 ). Therefore mCherry and mCherry $_{\text {cys }}$ were recombinantly expressed and purified and both proteins were freshly reduced with 10mM DTT. Note that mCherry itself does not contain any cysteins.

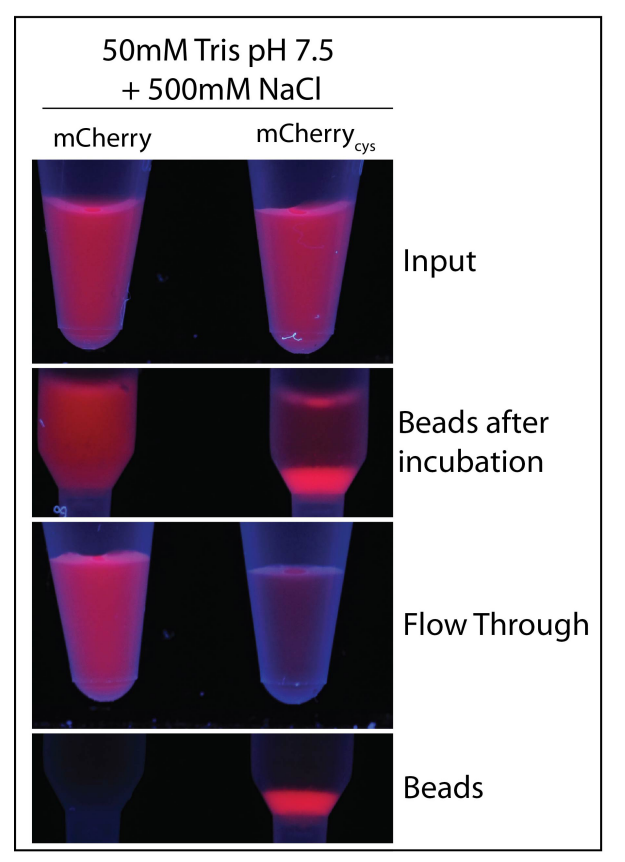

Figure 2.15: MADA matrix is highly specific towards reduced cysteins

$500 \mu \mathrm{l} 5 \mu \mathrm{M}$ mCherry or mCherrycys solution (recombinantly expressed, purified and freshly reduced with $10 \mathrm{mM}$ DTT prior to use) were incubated with $50 \mu \mathrm{l}$ MADA 2B Sepharose. Input: $5 \mu \mathrm{M}$ protein diluted in $5005 \mu \mathrm{M}$ buffer (50mM Tris/HCl $\mathrm{pH} 7.5,500 \mathrm{mM} \mathrm{NaCl}$ ); Beads after incubation: settled beads after $1 \mathrm{~h}$ incubation at RT; Flow Through: non-bound fraction after incubation; Beads: MADA 2B Sepharose after washing

Next, $5 \mu \mathrm{M}$ of each protein was diluted in $500 \mu \mathrm{l}$ buffer $(50 \mathrm{mM}$ Tris $/ \mathrm{HCl} \mathrm{pH} 7.5,500 \mathrm{mM}$ $\mathrm{NaCl}$, Input) and added to $50 \mu \mathrm{l}$ MADA 2B Sepharose. After 1h incubation at RT, a covalent binding of mCherry cys $_{\text {to }}$ the beads was observed, whereas mCherry lacking the Cterminal cystein did not show accumulation on the beads (Beads after incubation). Hence, the collected flow through shows a high fluorescence in case of mCherry, whereas only a 


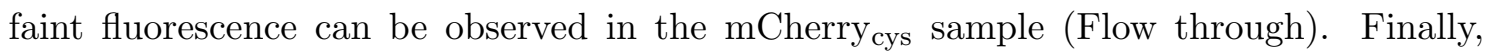

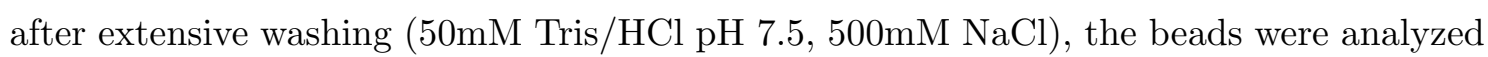

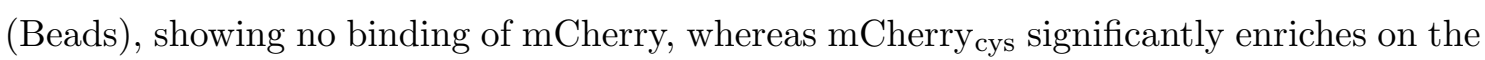
beads.

This data shows that the covalent coupling of proteins to the matrix happens exclusively via the reduced cysteins.

\subsubsection{Evaluation of coupling under various conditions}

The coupling ability of the MADA 2B Sepharose towards proteins containing reduced cysteins should ideally tolerate a variety of buffer system. Therefore, the initial experiment was repeated using three different buffers: the previously tested Tris-based buffer $(50 \mathrm{mM}$ Tris/ $\mathrm{HCl} \mathrm{pH} \mathrm{7.5,} \mathrm{500mM} \mathrm{NaCl),} \mathrm{a} \mathrm{potassium} \mathrm{phosphate-based} \mathrm{buffer}\left(20 \mathrm{mM} \mathrm{K} \mathrm{K}_{2} \mathrm{HPO}_{4}\right.$ pH7.4, 500mM NaCl) and a Hepes-based buffer system (20mM Hepes/KOH pH7.4, 500mM $\mathrm{NaCl}$ ). The salt concentration was kept constant at $500 \mathrm{mM} \mathrm{NaCl}$. As shown in figure $2.16 \mathrm{~A}$ the coupling specificity towards proteins with reduced cysteins could be observed in all three tested buffer systems: mCherrycys covalently attaches to the resin whereas mCherry without a cystein does not attach.

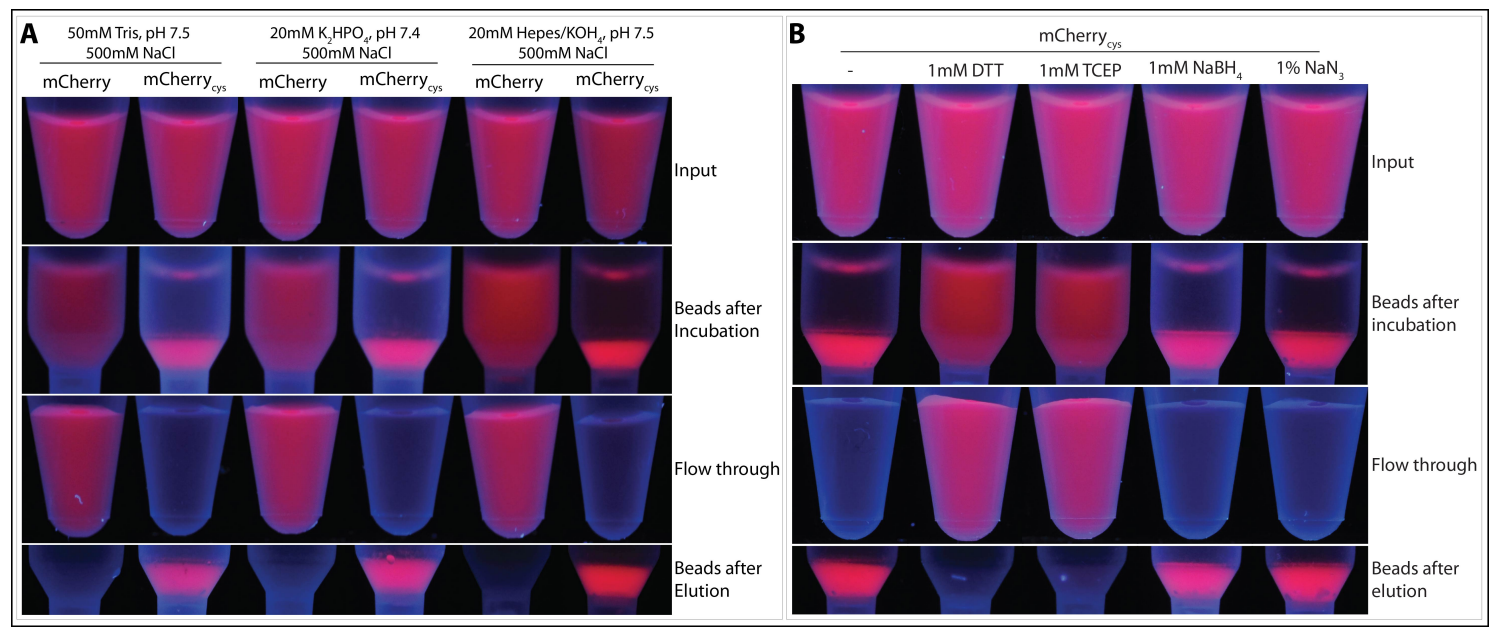

Figure 2.16: Evaluation of coupling under various conditions

(A): $500 \mu \mathrm{l} 5 \mu \mathrm{M}$ freshly reduced mCherry and mCherrycys were coupled to $50 \mu \mathrm{l}$ MADA resin under different buffer conditions. Pictures were taken from the Input, the beads after incubation, the Flow Through and the beads after washing. (B): $500 \mu \mathrm{l} 5 \mu \mathrm{M}$ freshly reduced mCherrycys was coupled to $50 \mu \mathrm{l}$ MADA resin in Tris buffer $(50 \mathrm{mM}$ Tris $/ \mathrm{HCl} \mathrm{pH} 7.5,500 \mathrm{mM} \mathrm{NaCl}$ ) containing various additives (1mM DTT, $1 \mathrm{mM}$ TCEP, $1 \mathrm{mM} \mathrm{NaBH}_{4}, 1 \%$ NaN 3 ). Pictures were taken from the Input, the beads after incubation, the Flow Through and the beads after washing.

It is known that even trace amounts of free DTT (Dithiothreitol) in the protein sample interfere with covalent attachment of the protein to a maleimide activated resin. DTT itself has a thiol group and thus can covalently attach to the maleimide itself (see figure $2.16 \mathrm{~B}$, second column). That means, after fresh reduction of the cystein using DTT, the sample needs to be rebuffered to a DTT free solution prior to coupling. To possibly avoid 
this time consuming step, it was tested, whether other reducing agents such as TCEP (Tris(2-chloroethyl) phosphate) or $\mathrm{NaBH}_{4}$ (Sodium borohydride) would permit coupling. In addition, the effect of sodium azide $\left(\mathrm{NaN}_{3}\right)$ was analyzed, due to the fact that the compound is frequently used as additive for long time storage of materials.

Figure 2.16B shows the corresponding coupling assay. The coupling reactions were performed in a Tris buffer system $(50 \mathrm{mM}$ Tris $/ \mathrm{HCl} \mathrm{pH} 7.5,500 \mathrm{mM} \mathrm{NaCl})$. As expected, $1 \mathrm{mM}$ DTT abolishes coupling of mCherry $_{\text {cys }}$ to the resin completely, as does the reducing agent TCEP by presumably reacting with the MADA double bond. However, neither $1 \mathrm{mM}$ $\mathrm{NaBH}_{4}$, which also has an reducing effect, nor the additive $\mathrm{NaN}_{3}$ hinder mCherrycys to covalently attach to the MADA 2B Sepharose.

To evaluate further tolerable coupling conditions, different salt concentrations and $\mathrm{pH}$ values will be tested in the future. Additionally, various conditions for long-term storage conditions of the MADA resin will be analyzed.

\subsubsection{MADA matrix capacity and background}

Next, the capacity of the MADA 2B Sepharose for direct coupling of ProteinA and streptavidin was analyzed as well as the unspecific binding of proteins to the resin upon incubation with lysate (figure 2.17).

First, a constant amount of MADA Sepharose $(20 \mu \mathrm{l})$ was incubated with increasing concentrations of Streptavidin $(0.5 \mu \mathrm{g} / \mu \mathrm{l}$ beads $-5 \mu \mathrm{g} / \mu \mathrm{l}$ beads $)$ diluted in $200 \mu \mathrm{l}$ Tris buffer $(50 \mathrm{mM}$ Tris/ $\mathrm{HCl} \mathrm{pH} 7.5,500 \mathrm{mM} \mathrm{NaCl})$. Input and flow through samples were analyzed by SDS-PAGE. Slight amounts of non-bound streptavidin can be detected in the flow through sample after addition of $5 \mu \mathrm{g} / \mu \mathrm{l}$ streptavidin. The other flow through samples do not contain any unbound protein (figure 2.17A). Hence, the MADA 2B Sepharose tested seems to have a capacity of $\sim 4 \mu \mathrm{g}$ streptavidin/ $\mu$ l beads.

Next, not only the capacity for the direct coupling of ProteinA to the MADA 2B Sepharose, but also the capacity for binding of the affinity partner (here IgGs) was analyzed. Therefore a constant amount of MADA 2B Sepharose $(20 \mu \mathrm{l})$ was first incubated with increasing amounts of freshly reduced ProteinA $(0 \mu \mathrm{g} / \mu \mathrm{l}$ beads $-5 \mu \mathrm{g} / \mu \mathrm{l}$ beads), followed by incubation with rabbit sera. The bound IgGs were eluted by SDS loading buffer and analyzed by SDS-PAGE (figure 2.17B). When looking at the flow through sample, it is obvious that the ProteinA sample used for coupling was not fully reduced. The non-bound fraction detectable in the first samples with rather low concentrations of ProteinA might resemble this non-reduced pool. Nonetheless, a significant portion of ProteinA is able to covalently attach to the beads (estimated by comparing Input and flow through sample intensity). With increasing ProteinA concentrations on the beads, the amount of IgGs pulled out increases as well (only IgG heavy chain is shown). Enrichment of IgGs reaches a plateau 


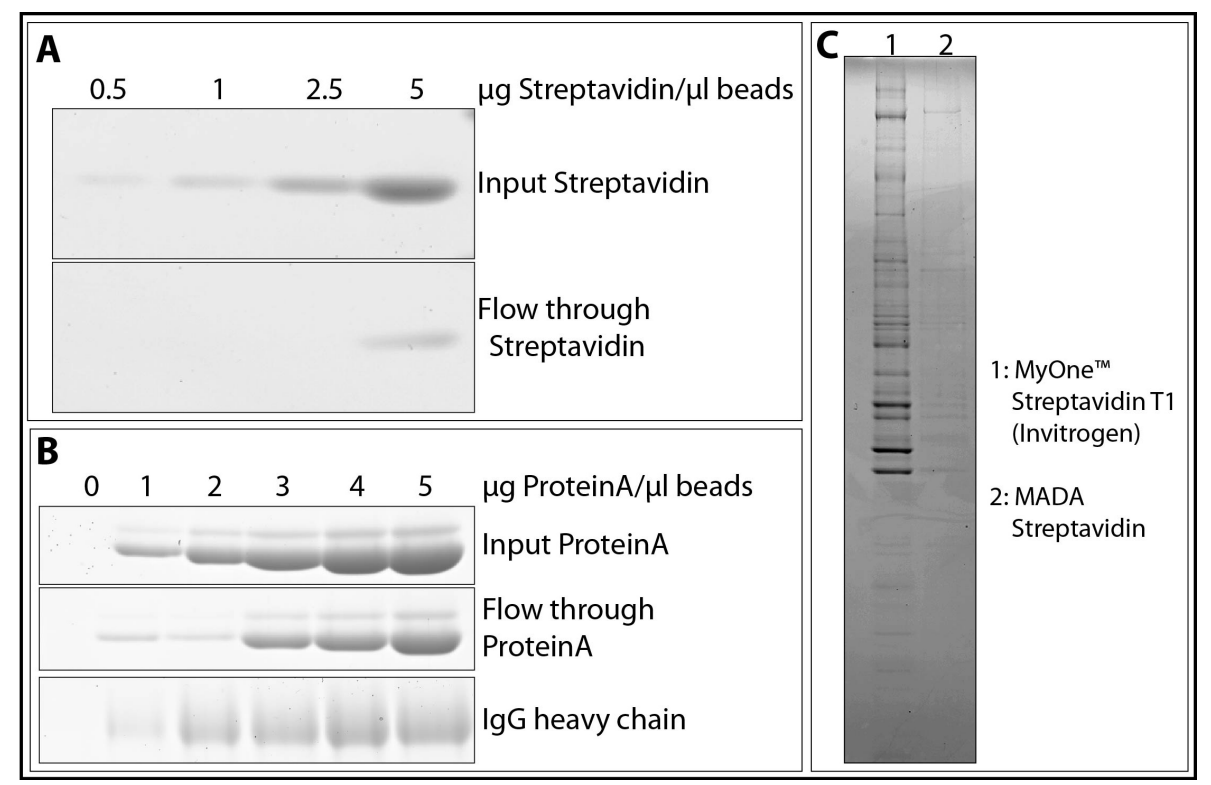

\section{Figure 2.17: MADA matrix capacity and background}

(A): Streptavidin with a C-terminal cystein was freshly reduced and coupled to the MADA resin in concentrations from 0.5 to $5 \mu \mathrm{g} / \mu \mathrm{l}$ beads. The input and flow through samples were loaded on SDS-PAGE to estimate the binding capacity of the resin. (B): ProteinA with a C-terminal cystein was freshly reduced and coupled to the MADA resin in concentrations from 0 to $5 \mu \mathrm{g} / \mu \mathrm{l}$ beads. The beads were further incubated with rabbit sera (diluted 1:3 with 1xPBS) in order to pull out IgGs. The binding capacity of ProteinA to the matrix was analyzed by loading input and flow through samples to SDS-PAGE. In addition, IgGs bound to the resin were eluted with SDS and analyzed by SDS-PAGE as well (only heavy chain shown here). (C): Commercially available magnetic streptavidin beads and MADA magnetic streptavidin beads were analyzed for their background binding when incubated with HeLa S10 lysate. (1) MyOne ${ }^{\mathrm{TM}}$ Streptavidin T1 (Invitrogen) (2) Streptavidin MADA magnetic beads quenched with $0.1 \mathrm{M}$ $\beta$-mercaptoethanol. All samples were analyzed by using gradient polyacrylamide gels.

at a concentration of $\sim 4 \mu \mathrm{g}$ ProteinA/ $\mu$ l MADA 2B Sepharose.

Finally, the background binding of proteins to the resin was analyzed. Here, two different streptavidin magnetic beads were incubated with HeLa S10 lysate (adjusted to 50mM

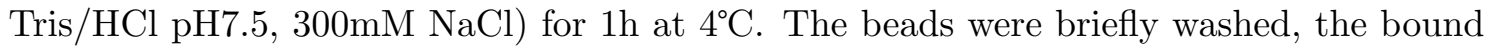
proteins were subsequently eluted with SDS loading buffer and analyzed by SDS-PAGE (figure 2.17C). The first lane shows the background binding on commercially available $\mathrm{MyOne}^{\mathrm{TM}}$ beads (Invitrogen). A significant number of proteins binds the resin unspecifically, which is disadvantageous when performing highly sensitive binding assays. Lane 2 represents the background binding of the HeLa cell lysate to streptavidin MADA magnetic beads generated in this study and quenched with $0.1 \mathrm{M} \beta$-mercaptoethanol after streptavidin immobilization. The resin shows significantly less background binding as compared to the commercial streptavidin beads. Remaining contaminants could possibly be further reduced by prolonging the washing procedure prior to elution.

In summary, the MADA chemistry introduced on Sepharose and magnetic beads shows a high specificity towards reduced cysteins. The resins show an adequate capacity towards its direct coupling partners and significantly less background binding as compared to com- 
mercial available products can be observed. These features are beneficial for biochemical approaches and thus can now be used for a wide range of different approaches.

\subsubsection{MADA matrix can be used for a variety of applications}

The chemistry for a MADA activated resin can be introduced to a variety of stationary phase substances such as silica, Sepharose or magnetic beads. Further, a multitude of different proteins can be covalently attached to the resin via thiol groups present in cystein side chains. This modularity allows the use of MADA resins in a broad range of applications. Some possible applications will be described in the following chapter.

\subsubsection{ZZ-Affibody MADA matrix shows less background as commercial IgG matrix}

A commonly used affinity tag system is ProteinA (or its engineered ZZ-domain) recognizing the Fc region of antibodies. Proteins fused to ProteinA or the ZZ-domain are hence able to bind IgG Sepharose, which acts as the stationary phase. However, commercial IgG Sepharose (GE Healthcare) shows strong background binding mainly under low salt conditions. Here, the commercial IgG Sepharose was compared with a ZZ-affibody MADA 2B Sepharose. The affibody ZpA963 binds a five-domain ProteinA fragment with an average $\mathrm{K}_{\mathrm{D}}=24 \mathrm{nM}$ and is further able to bind to each of the five domains individually with similar kinetics (Lindborg et al., 2013). The affibody was recombinantly expressed in E.coli and purified by affinity chromatography prior to coupling to the MADA resin by a reduced cystein introduced to the C-terminus of the affibody.

First, the two resins were incubated with E.coli lysate prepared with different salt concentrations (10-500mM NaCl in $50 \mathrm{mM}$ Tris/ $\mathrm{HCl} \mathrm{pH} \mathrm{7.5).} \mathrm{After} 1 \mathrm{~h}$ incubation, the beads were washed with the corresponding buffer and the unspecifically binding proteins were eluted with SDS loading buffer and analyzed by SDS-PAGE (figure 2.18A). IgG Sepharose shows significant unspecific background binding of proteins over a broad size range when incubated with low salt E.coli lysate $(10 \mathrm{mM} \mathrm{NaCl})$. The ZpA963 MADA resin in contrast shows less background binding. Bands can be observed however, in comparison to the IgG Sepharose these are significantly weaker. Nevertheless the unspecific binding must be further reduced to ideally end with an inert resin towards background binding. With increasing salt concentrations, the background binding of the IgG Sepharose decreases, however it still remains higher as compared to the ZpA963 MADA resin under all tested conditions. In addition, when eluting the bound proteins from the IgG Sepharose with SDS loading buffer, the IgGs heavy and light chain elute as well.

Next, the ZpA963 MADA Sepharose and the IgG Sepharose were compared in binding

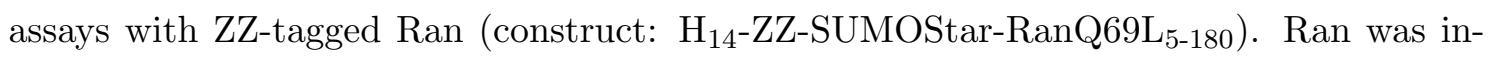




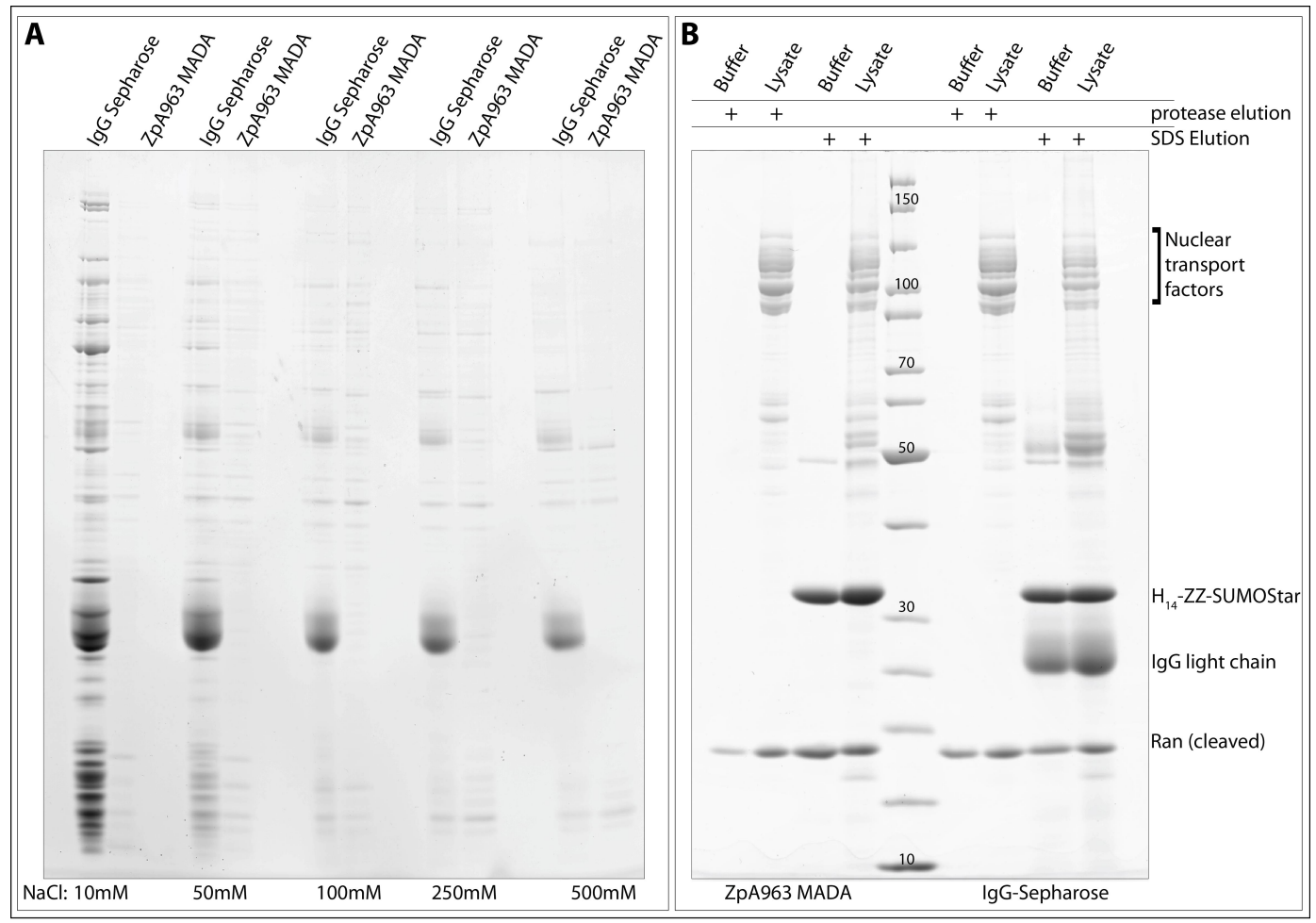

Figure 2.18: Comparing commercial IgG Sepharose and ZZ-affibody MADA Sepharose

(A): Background binding of E.coli lysate to either IgG Sepharose or ZpA963 MADA resin (affibody ZpA963 (Lindborg et al., 2013) recognizing the ZZ-domain was expressed and purified, reduced and coupled to MADA resin) was compared under different salt conditions $(10 \mathrm{mM}, 50 \mathrm{mM}, 100 \mathrm{mM}, 250 \mathrm{mM}$ and $500 \mathrm{mM} \mathrm{NaCl}$ in $50 \mathrm{mM}$ Tris/HCl, $\mathrm{pH7.5)}$. The proteins unspecifically binding to the resin were eluted with SDS and analyzed by SDS-PAGE. (B): Pull-out experiments were performed with IgG Sepharose (right panel) and ZpA963 MADA resin (left panel). $\mathrm{H}_{14^{-}}$ ZZ-SUMOStar-tagged Ran was incubated with HeLa S10 lysate (20mM Tris/HCl pH7.5, 100mM NaCl) and was subsequently pulled out using the two different resins. Elution was performed by protease elution and subsequent SDS elution (indicated with + ). All samples were analyzed by SDS-PAGE using gradient polyacrylamide gels.

cubated with HeLa S10 lysate (adjusted to 20mM Tris/HCl pH7.5, 100mM NaCl) and subsequently pulled out by either the affibody or the IgG Sepharose. The beads were washed with the low salt buffer, pre-eluted with SumoStar protease and subsequently eluted with SDS loading buffer. Both resins were able to efficiently pull out Ran and its binding partners (mainly nuclear transport receptors) from the lysate (see figure 2.18B). Elution with the protease results in a clean elution of cleaved Ran and the NTRs, the subsequent SDS loading buffer elution contains also other, presumably unspecifically binding proteins. However, the amount of unspecifically binding proteins seems to be higher when using IgGsSepharose. In addition, the IgG comes off the resin when elution is performed with SDS loading buffer resulting in a prominent band on the gel $(\sim 25 \mathrm{kDa})$ that might interfere with proper evaluation of data.

In conclusion, the ZpA963 MADA resin is more suitable for pull-out experiments or binding studies recognizing ZZ-tagged proteins. The efficiency of pulling out bait proteins and their associated binding partners is comparable to commercial available IgG Sepharose, nevertheless the background binding to the affibody MADA resin is significantly lower as 
compared to IgG Sepharose. This can be observed mainly under low salt conditions which are often required when analyzing low affinity interactions between proteins.

\subsubsection{ProteinA MADA matrix can be used for IP experiments}

Immunoprecipitation (IP) experiments are a powerful tool to analyze the composition and binding interaction of native complexes in vitro. This approach usually requires a ProteinA stationary phase to which IgGs against the target protein are covalently attached (for experimental details see 4.2.5.4). The beads are then incubated with a lysate of choice containing the target proteins. After extensive washing, the IgG bound proteins are eluted by low pH buffers. During this study, IP experiments were performed to analyze the composition of the endogenous wheat eIF3 complex. The stationary phase used during these experiments was $4 \mathrm{~B}$ Sepharose activated with maleimide. ProteinA was attached via its reduced cystein. However, unspecific background binding to the resin was high and the wheat extract needed to be pre-depleted by ProteinA Sepharose without attached antibodies to result in final elution samples that could be further analyzed by mass spectrometry (see figure 2.5). The additional depletion step should be neglectable when using the new MADA 2B Sepharose, as it was previously shown to have significantly less background binding. To test this aspect, ProteinA was covalently coupled to the MADA 2B Sepharose and subsequently antibodies against wheat initiation factors were attached. IP experiments were performed without any pre-depletion of the extract and the resulting elution samples were analyzed by SDS-PAGE and mass spectrometry (see figure 2.19).

IP experiments were performed using antibodies against wheat eIF1, eIF2 $\alpha$ and $\operatorname{eIF} 2 \mathrm{~B} \beta$. In all samples, the target protein was efficiently pulled out from the extract. eIF1 does not co-precipitate in a stochiometric manner with any other protein, which was also expected as eIF1 is not known to stably interact in multisubunit complexes. When pulling out eIF $2 \alpha$ from the wheat germ extract, stochiometric co-precipitation of eIF $2 \beta$ and eIF $2 \gamma$ was observed. The three subunits form the naturally occurring, stable eIF2 complex. When pulling out eIF2B $\beta$ from the extract, the associated complex, comprised out of 5 subunits $(\mathrm{eIF} 2 \mathrm{~B} \alpha-\mathrm{eIF} 2 \mathrm{~B} \epsilon)$ can be co-purified in a stochiometric manner. Additional bands in the eIF $2 \alpha$ and the eIF2B $\beta$ IP experiment can be observed. These were identified to be ribosomal proteins (marked with asterisks, figure 2.19). It needs to be further elucidated if these proteins do interact with the resin in an unspecific manner of if they indeed interact with the translation complexes precipitated. Same holds true for the faint smir that can be observed at around $\sim 60 \mathrm{kDa}$.

Although a certain level of unspecific binding could be observed, the purity of the elution samples greatly increased in comparison to previously performed IP experiments by switching from $4 \mathrm{~B}$ maleimide Sepharose to $2 \mathrm{~B}$ Sepharose using the MADA activation chemistry 


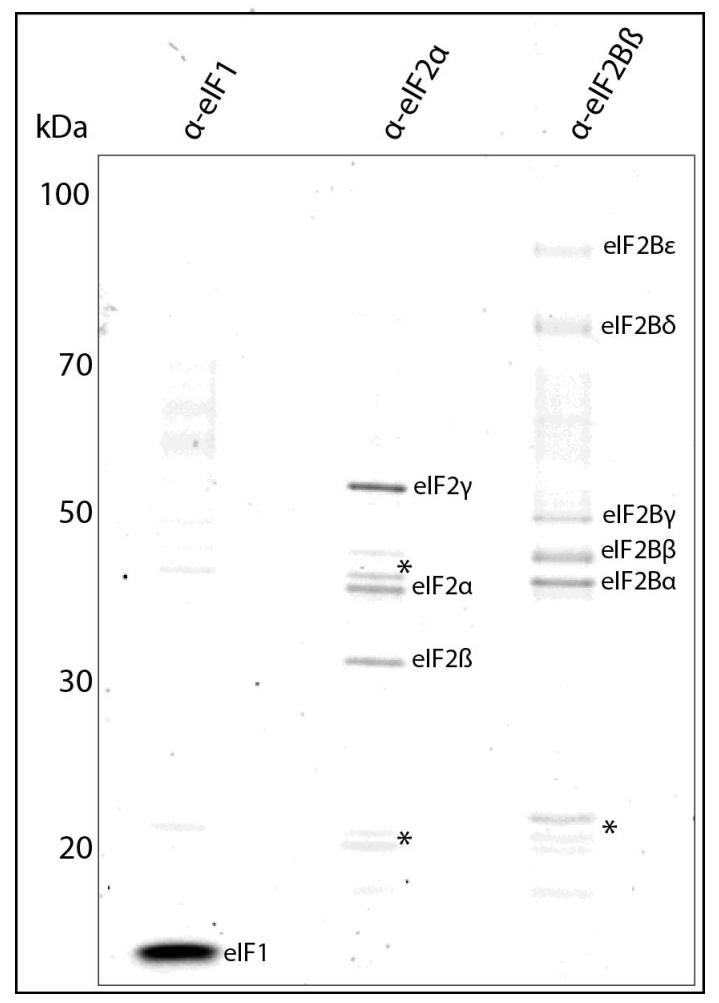

Figure 2.19: IP experiments with MADA ProteinA Sepharose

Freshly reduced recombinant ProteinA was coupled to the MADA 2B Sepharose in a concentration of $3 \mu \mathrm{g} / \mu \mathrm{l}$. Subsequently, IgGs were covalently attached as described in 4.2.5.3 and IP experiments were performed as described in 4.2.5.4. Here, antibodies against eIF1, eIF2 $\alpha$ and eIF2B $\beta$ were used. Samples were analyzed by SDS-PAGE ( $12 \%$ polyacrylamide gel) and stained using Colloidal Coomassie. The bands were identified by mass spectrometry. Bands marked with asterisks were identified as ribosomal proteins.

(see figure 2.5). Pre-clearing of the wheat germ extract was not required. However, the captured proteins or complexes are eluted from the IgGs by acidic elution, precipitated and then analyzed further. A purification of complexes under native conditions is not applicable by this method.

\subsubsection{Large complexes can be pulled out by MADA matrix}

A major advantage of MADA activated resins is that potentially every protein can be covalently attached to it as long as it contains an exposed and reduced cystein that is not functionally relevant in context of the protein itself or interactions of the protein towards other polypeptides. Here, this feature is exploited with the aim to purify large complexes in a native manner from lysates (in contrast to the IP technique, where proteins are eluted under extremely acidic conditions). Figure 2.20A shows the designed construct that had been attached to the MADA 2B Sepharose in the following experiment. It contains an N-terminal His-tag for purification from E.coli lysate, followed by a binding domain and a protease cleavage site. At the very C-terminus, an exposed cystein is located for covalent attachment to the resin. The binding domain can be varied depending on the individual 
approach, e.g. a ZZ-domain recognizing IgGs or the ZZ-affibody recognizing ZZ-tagged proteins or complexes. The protease cleavage site is required for elution of the bound proteins by specific protease cleavage.

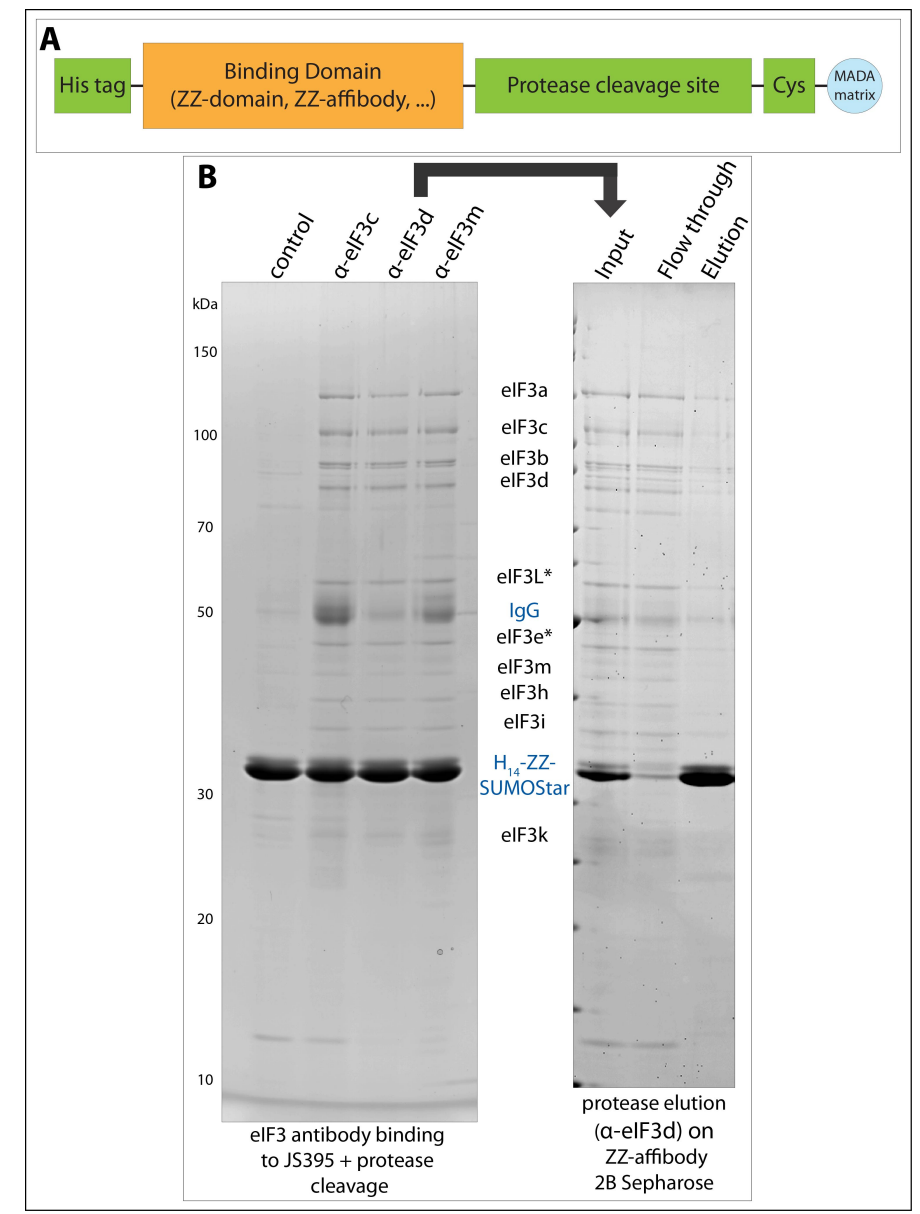

Figure 2.20: Large complexes can be pulled out by MADA magnetic beads

(A): Schematic representation of the construct covalently attached to the 2B MADA Sepharose in B. The N-terminal His tag was used for affinity purification of the construct. The following binding domain can be either ZZ-domain, the ZZ-affibody (ZpA963) or streptavidin. This domain enables to pull-out the target protein from an extract. Next, a protease cleavage site for specific protease elution was included. The C-terminal cystein allows efficient coupling to the MADA resin. (B): To pull out large complexes such as eIF3 from wheat extract, the affinity construct containing a ZZ-domain as binding domain was coupled to the MADA resin. In parallel, WGE was incubated with antibodies against eIF3c, eIF3d and eIF3m. Subsequently, the IgG supplemented WGE was incubated with the beads. After thorough washing, the elution via SUMOStar cleavage followed. Elution samples were taken and analyzed by SDS-PAGE. To remove the remaining parts of the affinity construct, the $\alpha$-eIF3d elution (Input) was loaded on ZZ-Affibody MADA Sepharose. The flow through should now contain the complex, the remaining tag should attach to the column. Input, Flow through and SDS elution samples were analyzed by SDS-PAGE. eIF3 complex pull out can be seen in the left panel, the removal of the tag in the right panel. JS395 expression construct: $\mathrm{H}_{14}$-ZZ-domain-SUMOStar-Spacer-Cys

As proof of principle, the system was used to purify the $\sim 800 \mathrm{kDa}$ eIF3 complex from the wheat germ extract. The binding construct, containing the ZZ-domain as binding domain, was covalently coupled to the MADA 2B Sepharose. In parallel, the wheat germ extract was supplemented with antibodies raised against eIF3c, eIF3d and eIF3m. Note that the antibodies were not covalently attached to the resin as in IP experiments. After incubation the binding construct coupled MADA resin was incubated with the IgG 
supplemented WGE. After 1h incubation, the beads were thoroughly washed, elution was performed using SUMOStar protease and the samples were analyzed by SDS-PAGE. The SUMOStar cleavage site was used in this experiment due to the fact that it can not be cleaved by any endogenous proteases in the extract (Panavas et al., 2009). Note that the complexes were eluted in neutral $\mathrm{pH}$ Tris buffer and thus can be potentially used for any further experiments right away.

Figure 2.20B (left panel) shows the protease elution fractions. The control sample represents the pull-out from wheat germ extract, which was not supplemented with IgGs. The background is significantly low. The predominant band at $\sim 30 \mathrm{kDa}$ represents the cleaved tag: $\mathrm{H}_{14}$-ZZ-SUMOStar. When the wheat germ extract was supplemented with antibodies recognizing single subunits of the eIF3 complex, the entire complex can be co-purified in a stochiometric manner. Again, the cleaved binding construct can be seen in the elution as well. Note the $\operatorname{IgG}$ heavy chain running at $\sim 50 \mathrm{kDa}$.

In order to remove the remaining binding construct ( $\mathrm{H}_{14}$-ZZ-SUMOStar), the elution fraction was incubated with the ZZ-affibody MADA resin, described in 2.4.3.1. The ZZ-domain binds to the resin, whereas the eIF3-IgG complex passes the resin without binding. This can be seen in figure 2.20B (right panel). The amount of binding construct is significantly reduced in the flow through fraction, whereas it enriches on the beads as shown in the elution fraction (SDS loading buffer).

Here, a system is presented to specifically pull out large complexes from a cell lysate in a native manner by affinity chromatography. We suggest that the system is not yet at its limit and can be further exploited to purify even larger objects such as ribosomes. These can be genomically tagged with a ZZ-domain, respectively, and can then be pulled out directly by a binding construct containing the ZZ-affibody. In principle, entire organelles such as mitochondria can be natively affinity purified from a lysate by using the MADA resin to which a suitable binding construct is covalently attached.

\subsubsection{MADA - Streptavidin matrix}

Streptavidin is a $16 \mathrm{kDa}$ protein naturally produced by the bacteria Streptomyces avidinii and able to bind biotin with an affinity of $K_{D}=10^{-14} \mathrm{M}$ (Tausig and Wolf, 1964; Green, 1990). Due to this very strong interaction streptavidin became a powerful tool for the purification and characterization of biotinylated substrates. In the following experiment, streptavidin was covalently attached to the MADA 2B Sepharose.

Streptavidin, containing an exposed C-terminal cystein, was expressed in E.coli and further purified. After coupling the protein to the MADA 2B Sepharose, the resin was incubated with either E.coli lysate alone to analyze the background binding or with E.coli lysate supplemented with in vivo biotinylated Ataxin or Nup98 anchor domain (supplied 
by Metin Aksu and Tino Pleiner). Elution was performed using SDS loading buffer at $95^{\circ} \mathrm{C}$ to break the streptavidin:biotin bond.

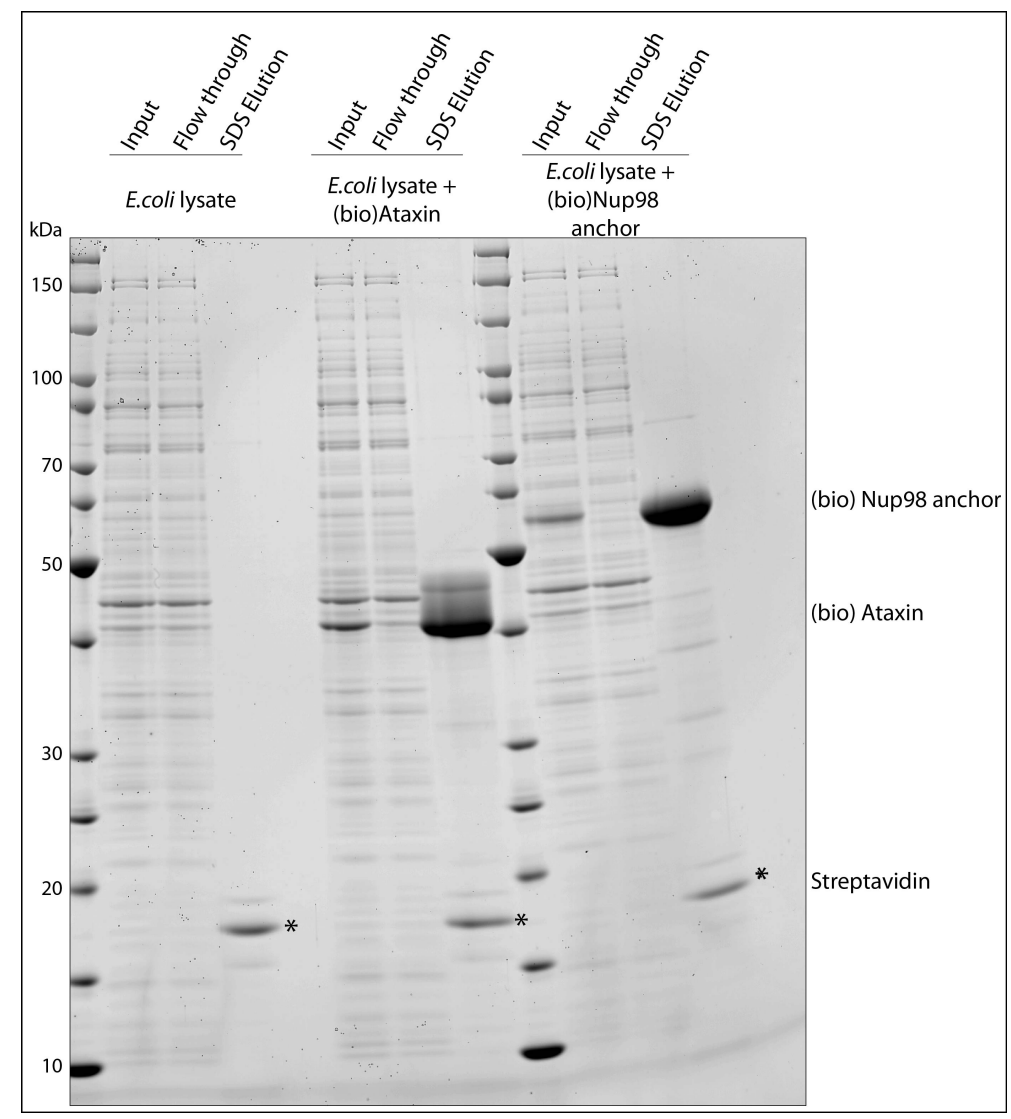

Figure 2.21: Streptavidin MADA Sepharose efficiently pulls out biotinylated bait from an extract Reduced streptavidin was coupled to MADA 2B Sepharose beads, which were incubated with either E.coli lysate only or E.coli lysate supplemented with in vivo biotinylated ataxin or Nup98 anchor domain. The beads were thoroughly washed after incubation and the bound proteins were eluted with SDS loading buffer at $95^{\circ} \mathrm{C}$. Samples were taken from the lysate input (Input) the flow through after $1 \mathrm{~h}$ incubation (flow through) and the SDS elution. The samples were analyzed by SDS-PAGE using gradient polyacrylamide gels. During elution, streptavidin leaks off the resin, marked with asterisks.

When the resin was incubated with E.coli lysate only, the elution sample contained only streptavidin running at $\sim 17 \mathrm{kDa}$ but no additional unspecifically binding proteins, which once more shows the low background binding of proteins to the resin (figure 2.21). The presence of streptavidin in the elution sample can be explained by the following: streptavidin forms a homo-tetramer. As soon as not all of the four subunits are covalently attached to the Sepharose, non covalent streptavidin subunits leak off the resin upon SDS elution. When E.coli lysate supplemented with biotinylated bait proteins (here Ataxin or the Nup98 anchor domain) was added to the beads, the bait proteins enriched on the beads and could be eluted from the resin. Similar experiments were also performed with streptavidin coupled to magnetic MADA beads, again showing specific enrichment of biotinylated proteins, whereas overall background binding remains low.

Although the streptavidin MADA 2B Sepharose performed extremely well as affinity resin 
for biotinylated proteins, it is however challenging to obtain large amounts of recombinantly expressed and purified streptavidin in a biotin free form, which is required to produce streptavidin matrix in feasible amounts. Without an N-terminal fusion tag, streptavidin expresses in an insoluble manner. Fusion to larger, solubility enhancing tags such as IF2domain-MBP increase solubility (Sørensen et al., 2003), nevertheless the problem persists that upon purification under native conditions pre-bound biotin (present in E.coli) will be co-purified. When coupling at least partially biotin bound streptavidin to a resin for affinity purification, the capacity of the resin towards biotinylated bait proteins would be extremely low. Besides the problematic expression and purification of recombinant streptavidin, it additionally assembles to a tetrameric complex of streptavidin which could cause steric hinderance once immobilized to a resin and leads to leakage (see figure 2.21). Furthermore, proteins purified by streptavidin resins need to be chemically or enzymatically modified in order to introduce the biotinylation. Most common, a biotinylatioon site is introduced to the bait protein (e.g. Avi-tag), which can be either in vivo or in vitro biotinylated by the enzyme BirA (Schatz, 1993).

An ideal protein:ligand pair for affinity chromatography would meet the following parameters: (1) extremely high affinity towards each other (2) both individual molecules should be small and monomeric (3) expression and purification should be simple and result in large amounts of recombinant protein (4) no further modification should be required (5) the proteins should not contain disulfide-bonds, as these might complicate the coupling of the protein to the MADA resin. In the following section, an affinity pair fulfilling all these criteria will be presented.

\subsection{Barnase:Barstar as novel affinity tag system}

Barnase is an extracellular ribonuclease produced by the bacterium Bacillus amyloliquefaciens. It is a small, single chain protein with a molecular weight of $13 \mathrm{kDa}$ that does not contain any disulfide bonds or relies on any non-peptide components for proper folding and function. To protect itself towards the toxic effects of the ribonuclease, Bacillus amyloliquefaciens also produces an intracellular inhibitor specific towards Barnase, termed Barstar. Barstar is a single chain protein as well with a molecular weight of $11 \mathrm{kDa}$. It contains two cysteins residues that do not form a disulfide-bond. Barnase and Barstar form a 1:1 noncovalent complex, thereby completely preventing Barnase toxicity (reviewed in Hartley, 1989). The barnase:barstar complex is known to bind with extremely high affinities with a $\mathrm{K}_{\mathrm{D}}=10^{-14} \mathrm{M}$ (Schreiber and Fersht, 1993). So far, studies on the Barnase:Barstar complex were mostly performed by co-expression of the two proteins. The individual expression of Barnase in E.coli respectively, would cause lethality of the cells. 


\subsubsection{Identification of Barstar mutants as binding partner for enzymatically inactive Barnase versions}

To use Barnase and Barstar as an affinity tag pair, it is essential to produce both proteins separately. However, when expressed alone, the ribonuclease Barnase is highly toxic. The catalytic residues are Glu 73 and His 102 acting together in a general acid-base mechanism. By mutating one of the catalytic residues, the toxicity of Barnase is impaired and the protein can be expressed in E.coli even without its inhibitor. However, mutations of His 102 to other residues weakens the affinity towards barstar. When mutating the His102 residue to aspartic acid or glutamine, the binding affinity decreases to $\mathrm{K}_{\mathrm{D}}=1.3^{-10} \mathrm{M}$, replacement by alanine leads to a $K_{D}=7^{-9} \mathrm{M}$ (Hartley, 1993).

In order to detoxify Barnase, the catalytic histidine residue was mutagenized to alanine, phenylalanine, methionine or aspartic acid. To compensate for a decrease in binding affinity, a cysteins residue on Barstar $\left(\mathrm{Cys}_{42}\right)$ was mutated as well to either alanine, valine, isoleucine or lysine. Replacements by alanine served as control, introduction of hydrophobic residues to both proteins should provide a hydrophobic docking site, the introduction of the negatively charged aspartic acid and the positively charged lysine could potentially lead to the formation of a shielded salt bridge in the interior of the complex.

To find the strongest pair among these mutations, competition experiments were performed. For these, the barnase versions were either fused to $\mathrm{H}_{14^{-}}$or $\mathrm{H}_{14^{-}}$-brSUMO; the Barstar versions were fused to ZZ- or ZZ-brNEDD8 tags. All constructs were expressed in E.coli and resulted in lysates containing soluble protein. This already shows that the introduced mutations in Barnase are sufficient to prevent Barnase toxicity.

Figure 2.22 shows a competition experiment where $\mathrm{H}_{14}$-Barnase ${ }_{\mathrm{H} 102 \mathrm{D}}$ :Barstar complexes were pulled out by $6 \% \mathrm{Ni}^{2+}$-chelate $500 \AA$ silica. The complexes formed in an E.coli lysate mixture containing Barnase and different Barstar versions either fused to ZZ- or ZZ-brNEDD8 tag (the tags ensure a size difference of the constructs and thus a discrimination of two Barstar versions). As control, each Barstar version was individually incubated with the Barnase $\mathrm{H}_{102 \mathrm{D}}$, showing that all of them are able to bind Barnase $\mathrm{H}_{102 \mathrm{D}}$ (lane 1-8). Next, two Barstar versions were mixed and added. The version with a stronger affinity towards Barnase should outcompete the other and thus should be more enriched on the resin. For Barnase $\mathrm{H}_{\mathrm{H} 102 \mathrm{D}}$, Barstar ${ }_{\mathrm{C} 42 \mathrm{~K}}$ shows the strongest binding. In presence of this mutant, all other versions can not be pulled out by Barnase. Barstar ${ }_{\mathrm{C} 42 \mathrm{~A}}$ and Barstar $_{\mathrm{C} 42 \mathrm{~V}}$ bind equally strong, whereas Barstar ${ }_{C 42 \mathrm{I}}$ is outcompeted by all other mutations. We assume that the isoleucine residue might sterically clash upon complex formation, still enable Barstar $_{\mathrm{C} 42 \mathrm{I}}$ to bind but disfavored in comparison to the other Barstar variants.

The same experiments were performed with the Barstar mutants on the other Barnase versions with the following results: 


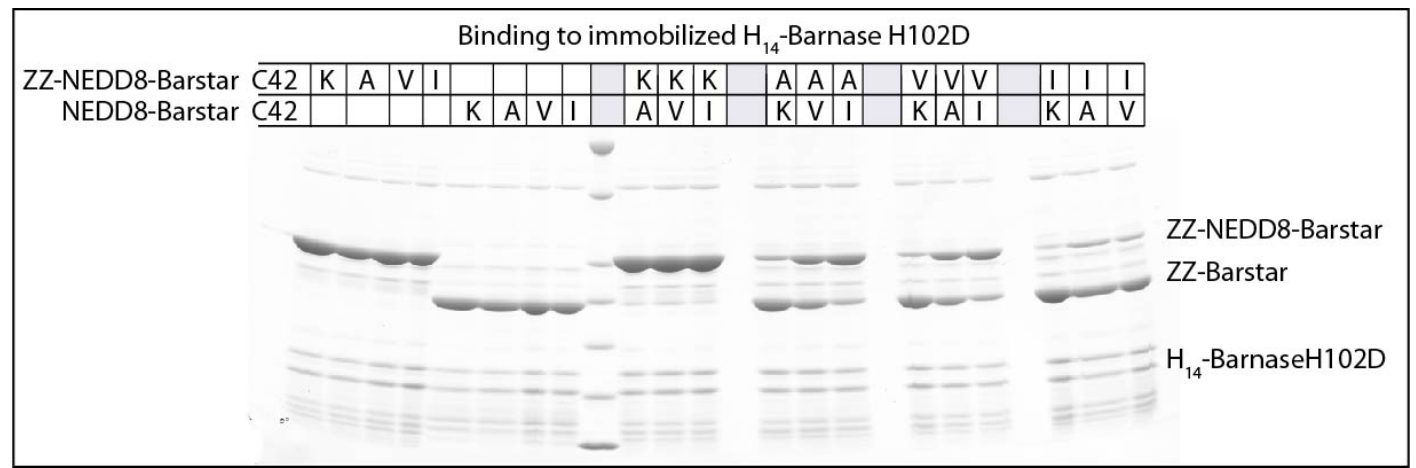

Figure 2.22: Barnase $\mathrm{H}_{\mathrm{H} 102 \mathrm{D}}$ and Barstar $_{\mathrm{C} 42 \mathrm{~K}}$ as ideal affinity pair

E.coli lysate containing $\mathrm{H}_{14}$-Barnase ${ }_{\mathrm{H} 102 \mathrm{D}}$ was incubated with lysates containing various Barstar mutants $(\mathrm{C} 42$ mutated to either K, A, V or I) tagged with ZZ-brNEDD8-tag or ZZ-tag. The formed complexes were bound to $6 \%$ $\mathrm{Ni}^{2+}$-chelate $500 \AA$ silica and eluted with SDS loading buffer. The Barstar mutants were either incubated separately with the Barnase lysate or two Barstar versions were added simultaneously to see which mutant has a higher affinity towards the Barnase. All samples were analyzed by SDS-PAGE.

Barnase $_{\mathrm{H} 102 \mathrm{~F}} \quad$ Barstar $_{\mathrm{C} 42 \mathrm{~V}}>$ Barstar $_{\mathrm{C} 42 \mathrm{~A}}>$ Barstar $_{\mathrm{C} 42 \mathrm{I}}$

Barstar $_{\mathrm{C} 42 \mathrm{~K}}$ does not bind

Barnase $_{\mathrm{H} 102 \mathrm{M}}$ No difference between Barstar $_{\mathrm{C} 42 \mathrm{~V}}$, Barstar ${ }_{\mathrm{C} 42 \mathrm{~A}}$ and Barstar $_{\mathrm{C} 42 \mathrm{I}}$

Barstar $_{\mathrm{C} 42 \mathrm{~K}}$ does not bind

Barnase $_{\mathrm{H} 102 \mathrm{~A}}$ Barstar $_{\mathrm{C} 42 \mathrm{~V}}>$ Barstar $_{\mathrm{C} 42 \mathrm{~A}}>$ Barstar $_{\mathrm{C} 42 \mathrm{I}}$

Barstar $_{\mathrm{C} 42 \mathrm{~K}}$ does not bind

Interestingly, Barstar ${ }_{\mathrm{C} 42 \mathrm{~K}}$ is only able to bind to Barnase if the histidine residue is mutated to an aspartic acid. Most likely, the positive charge on the lysine side chain and the negative charge on aspartic acid form a stable, shielded salt bridge in the protein complex interior, thereby drastically increasing the stability of the complex. When introducing hydrophobic amino acids to Barnase, the $\operatorname{Barstar}_{\mathrm{C} 42 \mathrm{~V}}$ mutant is slightly advantageous over the Barstar ${ }_{\mathrm{C} 42 \mathrm{~A}}$ version, possibly explainable by hydrophobic interactions on the complex interface.

Next, the salt sensitivity of the Barnase $\mathrm{H}_{\mathrm{H} 02 \mathrm{D}}$ and Barstar $_{\mathrm{C} 42 \mathrm{~K}}$ pair was analyzed. When using it as a tag system in affinity chromatography, a stable interaction even under high salt conditions is beneficial. The $\mathrm{H}_{14}$-tagged Barnase was immobilized on $6 \% \mathrm{Ni}^{2+}$-chelate $500 \AA$ silica and the beads were incubated with a lysate containing ZZ-brNEDD8-tagged Barstar. Complexes were formed in 50mM Tris pH7.5, 300mM NaCl. Next, the beads were continuously washed for $\sim 20$ minutes with buffer containing increasing salt concentrations $(300 \mathrm{mM} \mathrm{NaCl}-4.75 \mathrm{M} \mathrm{NaCl})$ and subsequently eluted with SDS loading buffer. As control, beads were only briefly washed with the $300 \mathrm{mM} \mathrm{NaCl}$ buffer and the complex was immediately eluted with SDS loading buffer (control). The elution samples were analyzed by SDS-PAGE to see if the complex dissociates with increasing ionic strength. Figure 
2.23 shows that even under very high salt conditions $(4.75 \mathrm{M} \mathrm{NaCl})$, the complex remains stable and both proteins can be eluted from the column as a stochiometric complex.

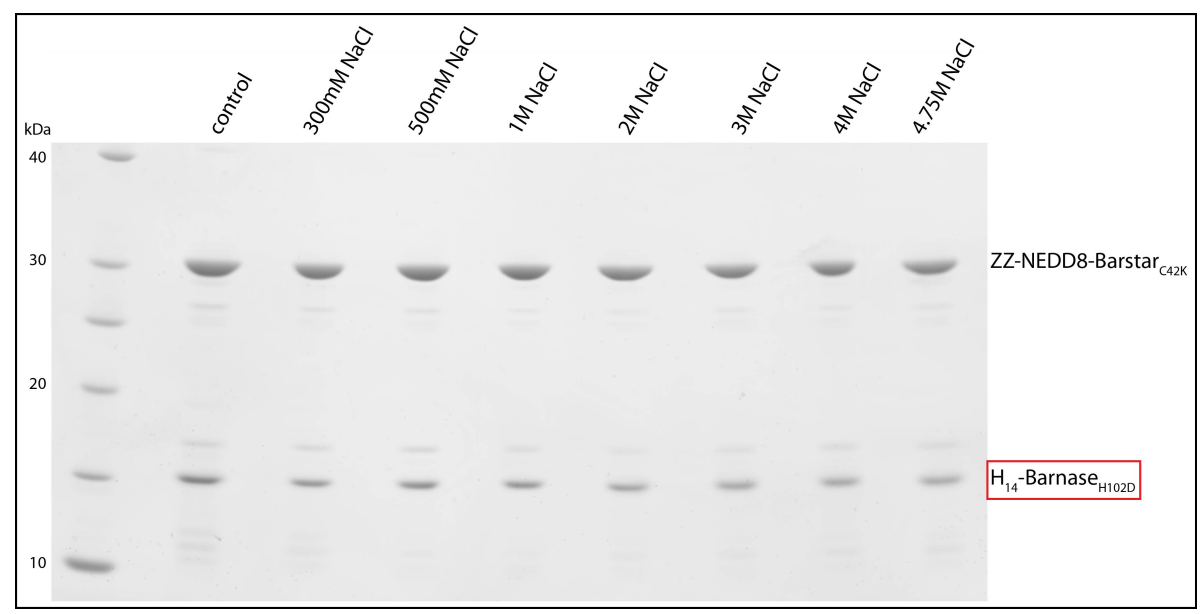

Figure 2.23: Barnase ${ }_{\mathrm{H} 102 \mathrm{D}}:$ Barstar $_{\mathrm{C} 42 \mathrm{~K}}$ complex is extremely salt tolerant

His-tagged Barnase ${ }_{\mathrm{H} 102 \mathrm{D}}$ was bound to $6 \% \mathrm{Ni}^{2+}$-chelate $500 \AA$ silica (red box). Subsequently, ZZ-brNEDD8 tagged Barstar $_{\mathrm{C} 42 \mathrm{~K}}$ was added and the complex was formed on the beads. After thorough washing with a buffer containing $300 \mathrm{mM} \mathrm{NaCl}$, the complexes were washed for 20 minutes (constant flow) with buffers containing increasing salt concentrations $(300 \mathrm{mM}-4.75 \mathrm{M} \mathrm{NaCl})$. As control, the beads were washed only once with $300 \mathrm{mM} \mathrm{NaCl}$ buffer and eluted immediately with SDS loading buffer (control). After washing, the remaining protein on the beads was eluted with SDS loading buffer. All samples were analyzed by SDS-PAGE using a gradient polyacrylamide gel.

In summary, the Barnase $\mathrm{H}_{102 \mathrm{D}}$ and Barstar $_{\mathrm{C} 42 \mathrm{~K}}$ pair seems to be a highly stable and thus well suited pair for further use as tag system and was therefore chosen to be further characterized.

\subsubsection{Structure determination of Barnase:Barstar complex}

To learn more about the Barnase ${ }_{\mathrm{H} 102 \mathrm{D}}$ :Barstar ${ }_{\mathrm{C} 42 \mathrm{~K}}$ complex, the two proteins were individually expressed in E.coli either tagged with $\mathrm{H}_{14}$-brSUMO or ZZ-brNEDD8. The lysates were mixed and purified according to Frey and Görlich (2014b), resulting in pure and stochiometric complexes as shown in figure $2.24 \mathrm{~A}$. The lysate mix was applied to $6 \% \mathrm{Ni}^{2+}$ chelate $500 \AA$ silica and elution was performed by SENP1 (SUMO Elution). The elution fraction was subsequently applied to the ZZ-affibody resin, to which the ZZ-tagged Barstar in complex with Barnase can bind. Non-complexed Barnase does not bind the resin and passes through the column. Elution was performed using NEDP1 protease (NEDD8 Elution). The pooled elution fractions, containing the stochiometric Barnase:Barstar complex were additionally purified by gel filtration using a SD200 16/60 (GE Healthcare) column. The gel filtration profile and the peak fractions on SDS-PAGE are shown in 2.24B. The two proteins form a stable complex as no dissociation of one component can be observed and the absorption peak shows a high symmetry. The complex containing fractions were pooled and concentrated to $10 \mathrm{mg} / \mathrm{ml}$ in $20 \mathrm{mM}$ Tris/HCl pH7.5, $100 \mathrm{mM} \mathrm{NaCl}, 1 \mathrm{mM}$ DTT. These samples were used by Dr. Sergei Trakhanov for successful crystallization trails. The 




Figure 2.24: Purifying the Barnase ${ }_{\mathrm{H} 102 \mathrm{D}}:$ Barstar $_{\mathrm{C} 42 \mathrm{~K}}$ complex

(A) Purification of a stochiometric and highly pure Barnase ${ }_{\mathrm{H} 102 \mathrm{D}}$ :Barstar ${ }_{\mathrm{C} 42 \mathrm{~K}}$ complex using a purification approach with two affinity chromatography steps and two orthogonal protease systems (Frey and Görlich, 2014b). $\mathrm{H}_{14}$-brSUMO-Barnase ${ }_{\mathrm{H} 102 \mathrm{D}}$ and ZZ-brNEDD8-Barstar ${ }_{\mathrm{C} 42 \mathrm{~K}}$ were individually expressed in E.coli. Samples were taken before induction(bi), after induction (ai), after lysis using sonication (aL) and from the supernatant after ultracentrifugation (aUZ). Subsequently, the two lysates were mixed (Input (Ni beads)) and incubated with 6\% $\mathrm{Ni}^{2+}$-chelate $500 \AA$ silica. The flow through was collected (Flow through) and elution was performed using $0.1 \mu \mathrm{M}$ SENP1 protease (SUMO Elution). The eluted fraction was subsequently incubated with ZZ-affibody MADA beads (Input (Affibody beads)), the flow through was collected (Flow through) and elution was performed with $1 \mu \mathrm{M}$ NEDP1 protease (NEDD8 Elution). This step was repeated due to high protein content in flow through fraction (flow through 2 and NEDD8 elution 2). The elution fractions were pooled and run over SD75 16/60 gelfiltration column (Input Gefi). The elution fractions were pooled (Gefi pool), concentrated and used for crystallization trials. (B) Gel filtration chromatogram and peak fractions on SDS-PAGE. 
complex crystallized in the following conditions and diffracted to a resolution of $1.98 \AA$ : $50 \mathrm{mM}$ BTP-citrate pH 7.1, 1.2M LiCl, 15\% PEG 6000. The structure was solved by Dr. Sergei Trakhanov by molecular replacement using the crystal structure published by Buckle et al. (1994) (pdb code: 1BRS). Crystallization details are presented in table 4.5.



Figure 2.25: Crystal structure of the Barnase ${ }_{\mathrm{H} 102 \mathrm{D}}:$ Barstar $_{\mathrm{C} 42 \mathrm{~K}}$ complex

The crystal structure of the Barnase $\mathrm{H} 102 \mathrm{D}_{\mathrm{D}}: \mathrm{Barstar}_{\mathrm{C} 42 \mathrm{~K}}$ complex was solved at a resolution of $1.98 \AA$ using molecular replacement. The Barnase molecule is marked in magenta, the Barstar molecule in green, Barstars $\alpha$-helix 2 is marked in yellow. The mutated residues $\left(\mathrm{H}_{102} \mathrm{D}\right.$ on Barnase and $\mathrm{C}_{42} \mathrm{~K}$ on Barstar) are represented as sticks, red marks the oxygen atoms, blue the nitrogen atoms. The distance between the residues was measured and is shown by the black dashed line.

Figure 2.25 shows the crystal structure of the Barnase ${ }_{\mathrm{H} 102 \mathrm{D}}$ :Barstar $\operatorname{Ca}_{\mathrm{C} 42 \mathrm{~K}}$ complex at a resolution of $1.98 \AA$. The Barnase molecule is presented in magenta, the Barstar protein in green. The mutated residues Asp $\mathrm{As}_{102}$ on Barnase and Lys 42 on Barstar are highlighted in white, showing the nitrogen atoms in blue and the oxygen atoms in red. In addition, the distance between the two side chains was measured to be $2.7 \AA$ and thus a close enough proximity to form a stabilizing salt bridge. The Barnase protein shows the characteristic antiparallel $\beta$-sheet and the $\alpha$-helix packed against one side of the sheet. The Barstar protein shows a parallel $\beta$-sheet and four $\alpha$-helix regions. $\alpha$-helix 2 (shown in yellow) reaches into the catalytic center of Barnase. In a wild type situation, this intrusion is known to block the ribonuclease activity of Barnase (see figure 1.7).

\subsubsection{Protein Evolution on Barstar}

For further experiments, the Barstar protein was fused to a variety of different N-terminal fusion tags, such as $\mathrm{H}_{14}$-brSUMO or $\mathrm{H}_{14}$-MBP-brSUMO, and subsequently expressed in 
E.coli. Surprisingly, these constructs lead to insoluble protein (data not shown). In previous trails Barstar was fused to ZZ- or ZZ-brNEDD8 resulted in high amounts of soluble protein.

For usage of the Barnase:Barstar pair as affinity tag system, it is of great importance that both proteins can be expressed to high levels in a soluble manner. Large amounts of recombinantly purified proteins will be required for coupling to the MADA resin but also when the protein as a fusion tag, it should support folding and thus solubility of the fusion partner. The Barnase protein fulfills these criteria, however, the Barstar protein requires further improvement.

\subsubsection{Specific mutations increase Barstar solubility without interfering with Barnase binding}

When looking closer at the obtained crystal structure, the very N-terminus of the Barstar molecule seems to be imperfectly packed. Hydrophobic residues that should be buried in the core of the protein are exposed more towards the outside and rather hydrophobic patches seem to be not properly shielded by the $\beta$-sheet. As previously discussed, proteins are more prone to aggregation, when these hydrophobic areas are solvent exposed.

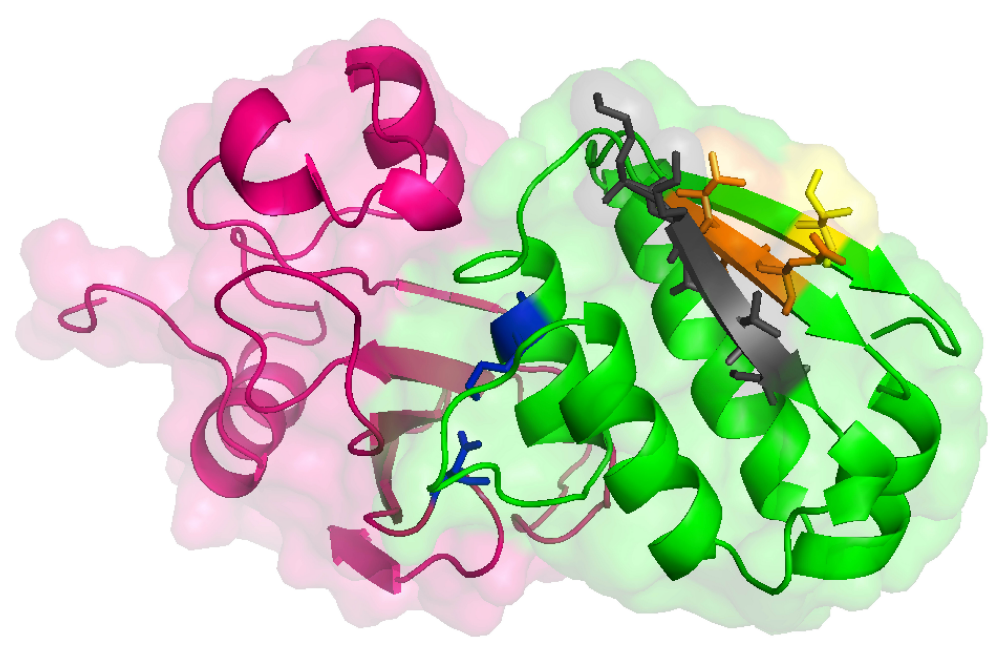

Figure 2.26: Mutations on Barstar $_{C 42 K}$ to increase solubility

To increase solubility of Barstar ${ }_{\mathrm{C} 42 \mathrm{~K}}$ various mutations at imperfectly packed regions were introduced: at the Nterminus (grey), a loop region (orange) and a single mutation of an isoleucin at the C-terminus (yellow). The Barnase molecule is highlighted in magenta, Barstar is drawn in green, the priorly mutated residues $\mathrm{H}_{102} \mathrm{D}$ on Barnase and $\mathrm{C}_{42} \mathrm{~K}$ on Barstar are shown in blue.

In order to decrease the amount of hydrophobics potentially facing the solvent, mutations were introduced by mutagenesis PCR. The following amino acid stretches were randomized (see also figure 2.26):

- the very N-terminal sequence MKKAV was mutated to GKKVT, GVKVT, GIKVT, GVKIT or GIKIT (grey) 
- the second strand in the parallel $\beta$-sheet has a VEY stretch that was mutated to either VEL, IEL or VEF in order to stabilize the overall $\beta$-sheet and to shield the underlying hydrophobic core of the protein (orange)

- at the very C-terminus of Barstar, a prominent Ile $\left(\mathrm{Ile}_{87}\right)$ points towards the solvent but seems misplaced in the structure. It was changed to a Glu residue (yellow)

Table 2.2 summarizes the solubility of the mutated Barstar versions. As previously described, the WT versions with an N-terminal MKKAV sequence and the C-terminal Ile results in insoluble protein. Neither changing only the $\mathrm{Ile}_{87}$ residue, nor mutating the N-terminal stretch to GVKVT resulted in soluble protein. However, when rendering the N-terminal stretch to GKKVT, the protein shows a moderate solubility. A high solubility was achieved when mutating the N-terminal stretch either to GVKVT or GIKIT and in addition mutating $\mathrm{Ile}_{87}$. Another possibility is to change the N-terminal stretch to GVKVT and in addition mutating the VEY stretch on one of the $\beta$-sheet strands to IEL instead.

Table 2.2: Mutations in Barstar slightly increase solubility

Different stretches on B.amyloliquefaciens Barstar were mutated with the aim to increase the solubility of the protein. The colored cells represent the various mutations or mutation combinations, the color code represents the solubility: insoluble (red), moderate solubility (yellow), high solubility (green). Note, that even for the soluble proteins, the effect depends on the optical density of the E.coli culture.

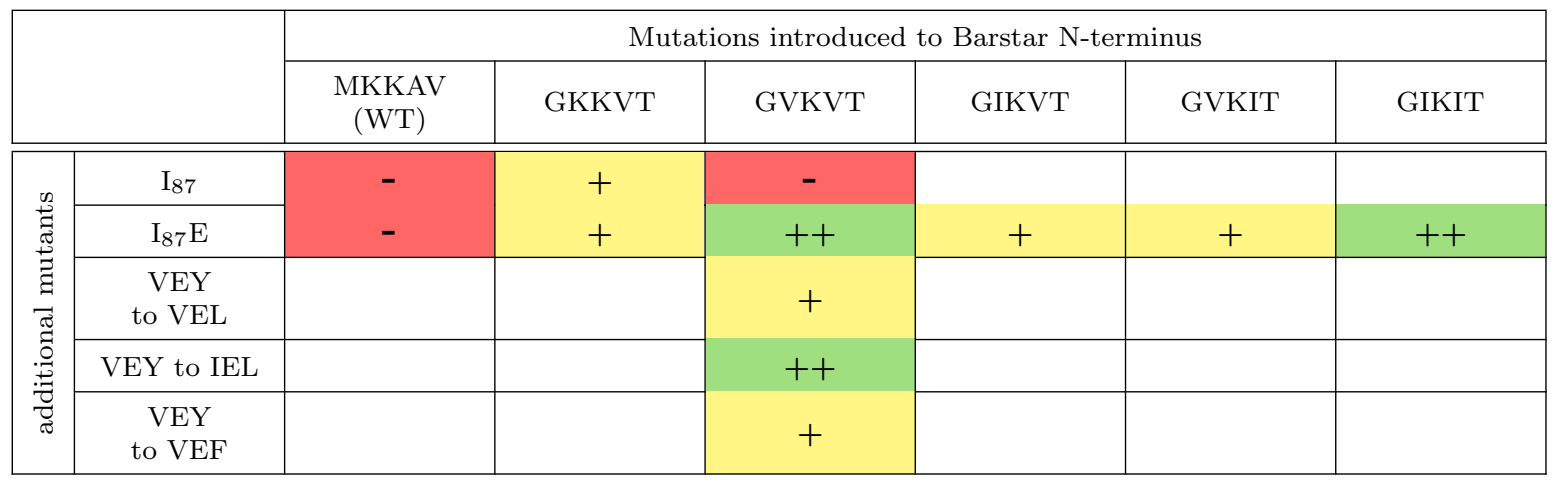

So far we can only speculate that by changing the position of hydrophobic side chains in the mutated stretches, these are buried in the protein interior and thus are less solvent exposed. This of course can only be validated by low result ion structure determination. Hence, Barstar containing a combination of beneficial mutations in complex with Barnase will be used in further crystallization trails.

Interestingly, soluble protein was only obtained when the E.coli culture did not grow to higher optical densities than a certain threshold $\left(\sim \mathrm{OD}_{600}=4\right)$. As soon as the cultures grew to higher densities, Barstar was expressed but no soluble protein could be obtained. 


\subsubsection{Phage Display}

With the aim to find a B.amyloliquefaciens Barstar sequence that results in a stably folding and well expressing soluble protein, a rather unbiased, second approach was taken. Instead of introducing mutations that in theory and according to the obtained structure should stabilize the protein, the Barstar protein was applied to random mutagenesis and subsequent analysis by Phage Display. The Barstar ${ }_{\mathrm{C} 42 \mathrm{~K}}$ protein as well as the Barstar mutations presented in 2.5.3.1 were randomly mutagenized. The resulting library was cloned into phagmids and a phage pool, displaying the Barstar variants as fusion to their coat protein pIII, was created (detailed protocol see 4.2.9). Prior, it was experimentally validated that Barstar can be displayed by phages (detection by Western Blot using $\alpha$ mouse pIII or $\alpha$-rabbit HA antibodies). In multiple rounds of panning, where phages able to bind to Barnase $\mathrm{H}_{\mathrm{H} 02 \mathrm{D}}$ are accumulated, the selection stringency was increased using decreasing concentrations of Barnase as binding partner (1nM - 1pM). The phagmids from the pool of bound phages were isolated and sequenced. Ideally, specific mutations would enrich throughout the sequences, representing a beneficial sequence/a beneficial protein compared to other sequences. Unfortunately, by analyzing the obtained sequences, no enrichment of specific mutation or combination of mutations could be identified.

\subsubsection{Barnase:Barstar complex from different species are advantageous compared to the $B$. amyloliquefaciens complex}

So far, the Barnase and Barstar proteins from Bacillus amyloliquefaciens were analyzed. The complex was expressed in E.coli, purified and crystallized. It was shown that the complex itself is very stable, as it does not dissociate on the gel filtration column and can tolerate up to $4.75 \mathrm{M}$ salt. The Barnase protein can be expressed and purified from E.coli. By contrast, the Barstar protein can not be solubly expressed in E.coli when fused to certain tags. Mutation of potential imperfect amino acid stretches only slightly increased the overall solubility, however, these variants can still not be purified to high amounts. In order to find a Barnase:Barstar pair, of which both proteins can be efficiently expressed and purified in isolation and can still form a highly stable complex, the Barnase and Barstar sequences from other bacterial species were examined.

\subsubsection{Barstar from different species show higher solubility than B.amyloliquefaciens Barstar}

Using the B.amyloliquefaciens Barstar sequence as starting point, similar proteins in other Bacillus sub-species were detected and analyzed by Protein BLAST (http://www.ncbi.nlm. nih.gov/Blast.cgi). The Bacillus pumilus and Bacillus plantarum Barstar sequences were chosen to be further analyzed. Bacillus plantarum itself has a variety of further sub- 
species, each containing slightly different Barstar sequences. Thus, two consensus sequences were designed and used for further analysis. In all Barstar variants, the cystein residue corresponding to $\mathrm{Cys}_{42}$ in Bacillus amyloliquefaciens was mutated to lysine to be able to bind the Bacillus amyloliquefaciens Barnase $_{\mathrm{H} 102 \mathrm{D}}$ with high affinity. The sequence comparison is shown in figure 2.27 , the mutated residues are boxed in yellow.

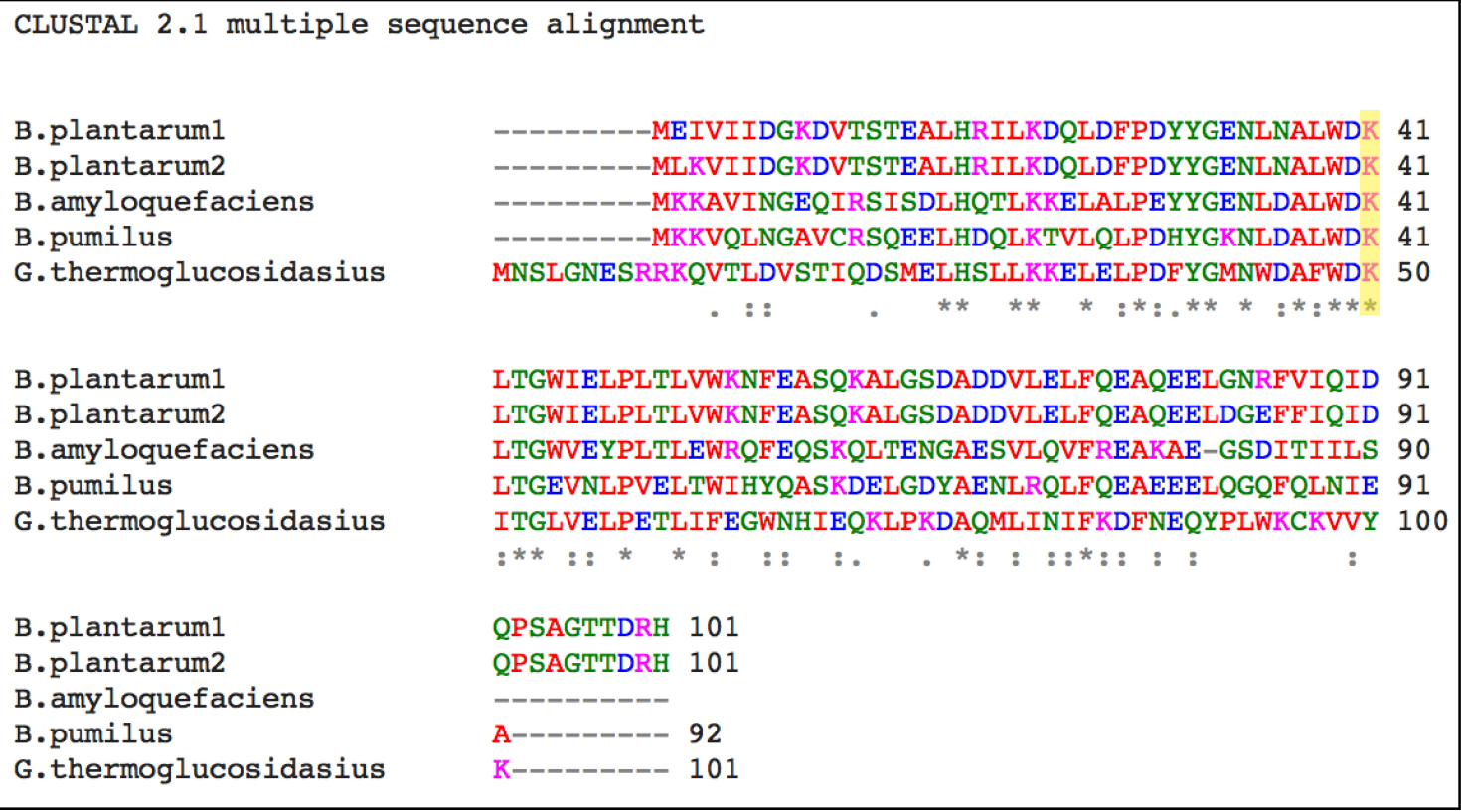

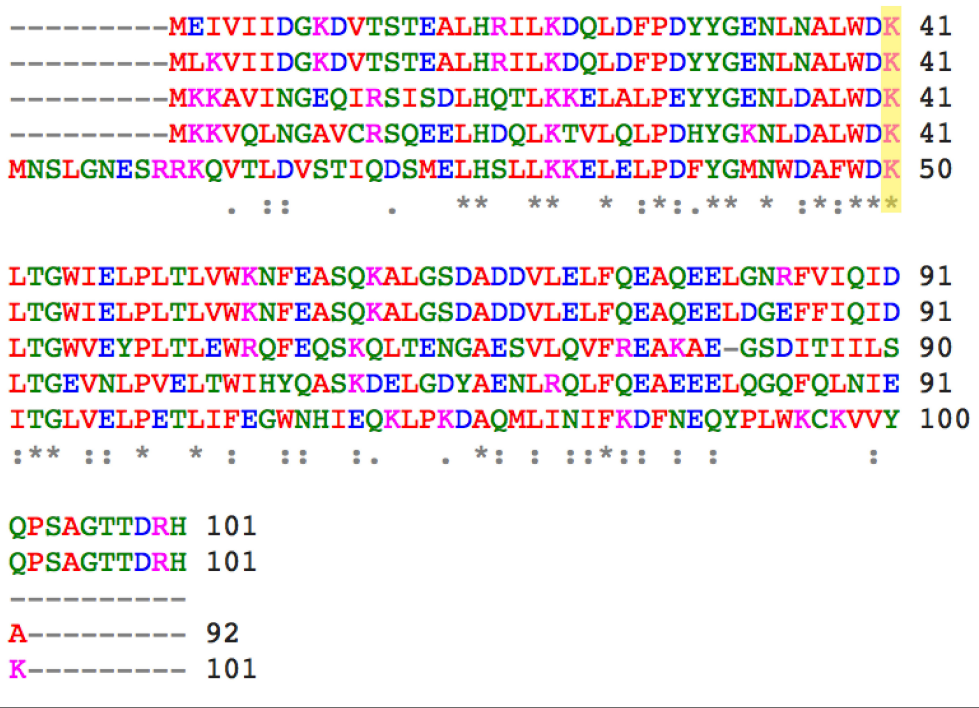

\section{Figure 2.27: Barstar sequence alignment}

Barstar protein sequences of B. amyloliquefaciens, B. pumilus, B.plantarum (here, two consensus sequences were designed) and $G$. thermoglucosidasius were aligned using the Clustal 2.1. multiple sequence alignment tool (http://www.ebi.ac.uk/Tools/msa/clustalw2). All sequences already contain the cystein to lysine mutation (aa 41 , respectively aa 50 for $G$. thermoglucosidasius, highlighted in yellow).

In addition, a more thermophil organism producing Barnase and Barstar proteins was analyzed. Proteins of thermophil organisms need to adapt to high temperatures and thus often have a more stable fold. Geobacillus thermoglucosidasius is a thermophilic aerobic or facultatively anaerobic bacterium, capable of growing at temperatures from $40^{\circ} \mathrm{C}$ to $70^{\circ} \mathrm{C}$ (Nazina et al., 2001). The Barstar and Barnase sequences were identified and the mutations corresponding to the B.amyloliquefaciens $\mathrm{H} 102 \mathrm{D}$ and $\mathrm{C} 42 \mathrm{~K}$ mutants were introduced.

The new Barstar and Barnase sequences was obtained by genesynthesis, subsequently cloned into $\mathrm{H}_{14}$-ZZ-brSUMO backbones and expressed in E.coli. After harvest, the cells were lysed by sonication and subsequently ultracentrifuged. The supernatant was analyzed by SDS-PAGE for the amount of soluble protein (for experimental details see 4.2.2). In general, all Barstar variants could be expressed to high levels in E.coli (figure 2.28). As previously observed, B.amyloliquefaciens Barstar can not be detected in the supernatant after ultracentrifugation, strongly suggesting that the protein is insoluble and thus precipitates during the centrifugation step. In comparison, the Barstar protein from 


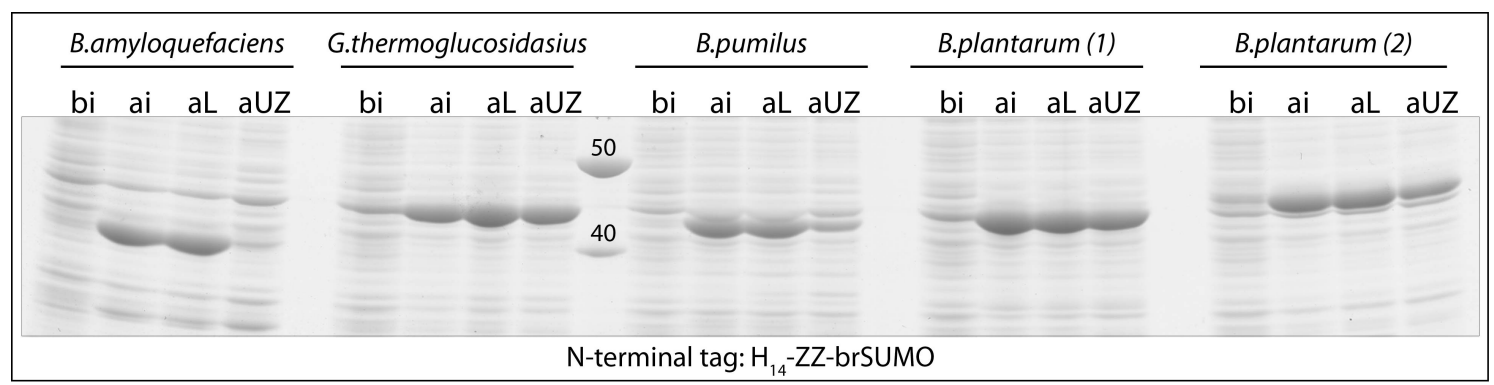

Figure 2.28: Barstars from various species show increased solubility

Barstar $_{\mathrm{C}}$ to $\mathrm{K}$ mutants from various species were expressed in E.coli and analyzed for their solubility. Samples were taken before induction (bi), after induction (ai), after lysis using sonication (aL) and from the supernatant after ultracentrifugation (aUZ). All samples were analyzed by SDS-PAGE using gradient polyacrylamide gels. Barstars from B.amyloliquefaciens, G.thermoglucosidasius, B.pumilus, and B.plantarum were analyzed. For B.plantarum, two different consensus sequences were designed. All proteins were expressed as $\mathrm{N}$-terminal fusion constructs to $\mathrm{H}_{14}$-ZZ-brSUMO.

G.thermoglucosidasius and the first B.plantarum consensus sequence are highly soluble, as nearly $100 \%$ of the expressed protein can also be found in the supernatant after centrifugation. The second B.plantarum consensus and the B.pumilus Barstar proteins can be partially expressed in a soluble manner.

It is worth mentioning that the E.coli cultures were grown to optical densities of $\mathrm{OD}_{600}=8.0$ and the Barstar proteins from other species than B.amyloliquefaciens could be (partially) expressed in a soluble manner. For the B.amyloliquefaciens Barstar and also its mutant forms (see 2.5.3.1) no soluble protein could be optioned when growing the E.coli cultures to an $\mathrm{OD}_{600}>4.0$.

In addition, binding assays were performed revealing that all new Barstar proteins are able to bind the B.amyloliquefaciens Barnase ${ }_{\mathrm{H} 102 \mathrm{D}}$ (data not shown).

\subsubsection{Geobacillus thermoglucosidasius Barnase}

The previous experiment showed that the G.thermoglucosidasius Barstar can be solubly expressed to high amounts in E.coli and can also bind the B.amyloliquefaciens Barnase protein. The G.thermoglucosidasius Barnase was also analyzed, as it most likely is an even better binding partner for the G.thermoglucosidasius Barstar. When looking at the sequence of G.thermoglucosidasius Barnase, an extended N-terminal sequence can be observed, which most likely serves as recognition stretch for the secretion of the extracellular ribonuclease. To find an ideal G.thermoglucosidasius Barnase construct, the protein was cloned with different $\mathrm{N}$-terminal truncations (see figure 2.29A) and expressed in E.coli to analyze the expression levels and the solubility of the constructs (all tagged to $\mathrm{H}_{14}$-brSUMO). The truncations were designed according to an alignment of the G.thermoglucosidasius Barnase sequence and the sequence of the B.amyloliquefaciens expression construct used so far. 


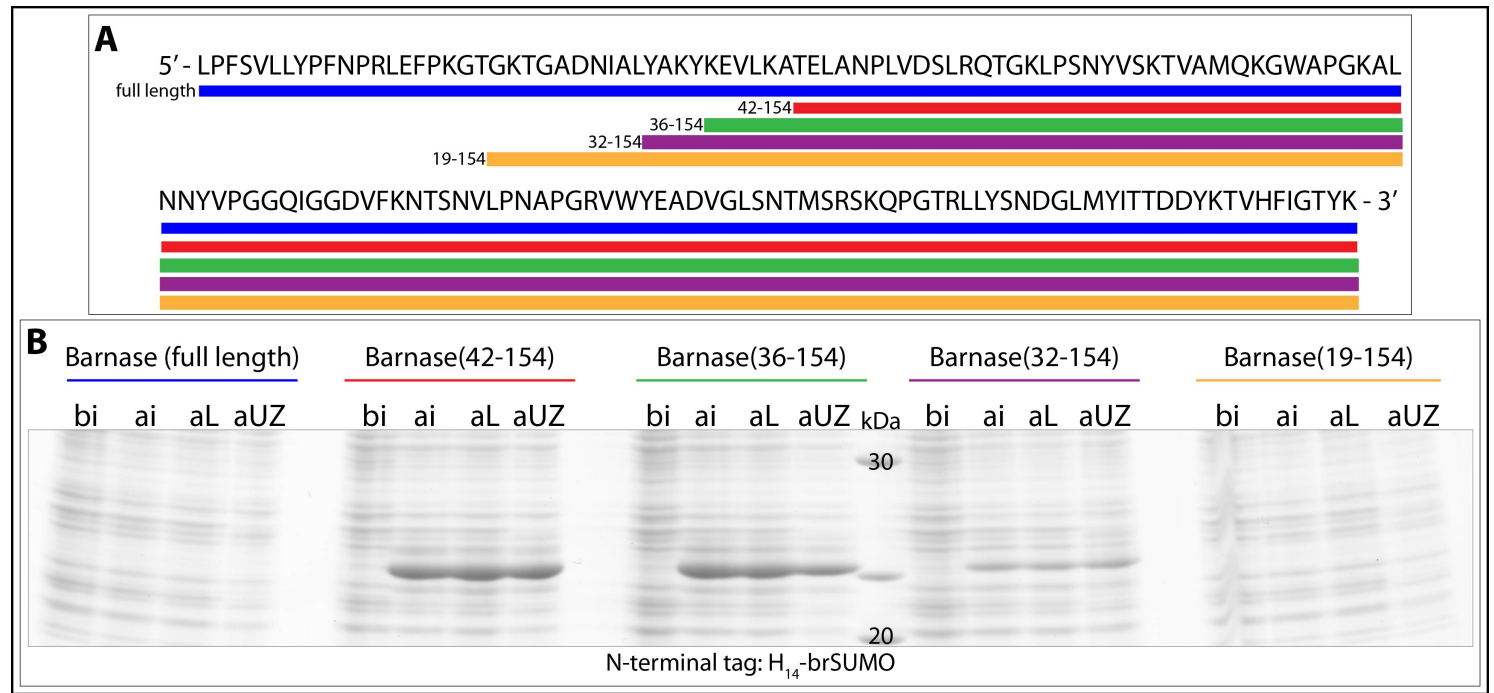

Figure 2.29: G.thermoglucosidasius Barnase versions

(A): Barnase $($ H108D) from G.thermoglucosidasius contains a pre sequence, which is cleaved off in living cells. In order to find a Barnase version that has a stable fold, various $\mathrm{N}$-terminal truncations were designed. (B): The truncated versions were expressed in E.coli and analyzed for their solubility. Samples were taken before induction (bi), after induction (ai), after lysis using sonication (aL) and from the supernatant after ultracentrifugation (aUZ) and analyzed by SDS-PAGE (gradient polyacrylamide gels). All proteins were expressed as N-terminal fusion constructs to $\mathrm{H}_{14}$-brSUMO.

Figure 2.29B shows the solubility assay of the various Barnase versions. Interestingly, the two constructs with the longest N-terminal extension (blue and yellow) failed to solubly express in E.coli. With shortening the N-terminal extensions, the expression levels for the constructs increase. The G.thermoglucosidasius versions G.t.Barnase ${ }_{(36-154)}$ (green) and G.t.Barnase ${ }_{(42-154)}$ (red) expressed to high levels and resulted in soluble protein. Thus, these two versions were further analyzed in order to create a G.thermoglucosidasius Barnase:Barstar pair for affinity chromatography.

\subsubsection{Geobacillus thermoglucosidasius complexes are highly stable}

To estimate the binding strength of the new Barstar versions towards the B.amyloliquefaciens Barnase $_{\mathrm{H} 102}$, competition assays were performed. As previously describes, the Barstar versions were expressed as either ZZ- or ZZ-brNEDD8- fusions proteins to create a difference in molecular weight. Next, the constructs were either incubated alone with the immobilized Barnase protein or in combinations. Figure 2.30 shows that all Barstar

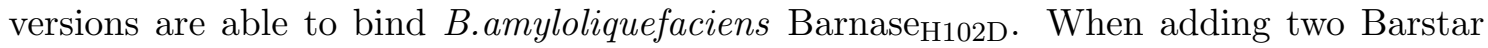
versions, the G.thermoglucosidasius Barstar has the highest affinity towards the Barnase protein, as it is able to compete out all other Barstar variants. The binding affinity of the B.pumilus and the two B.plantarum Barstar proteins towards Barnase are similar to each other.

Next, a variety of different Barnase:Barstar complexes was formed and purified as described previously for the B.amyloliquefaciens complex (see figure 2.24). The G.thermo- 


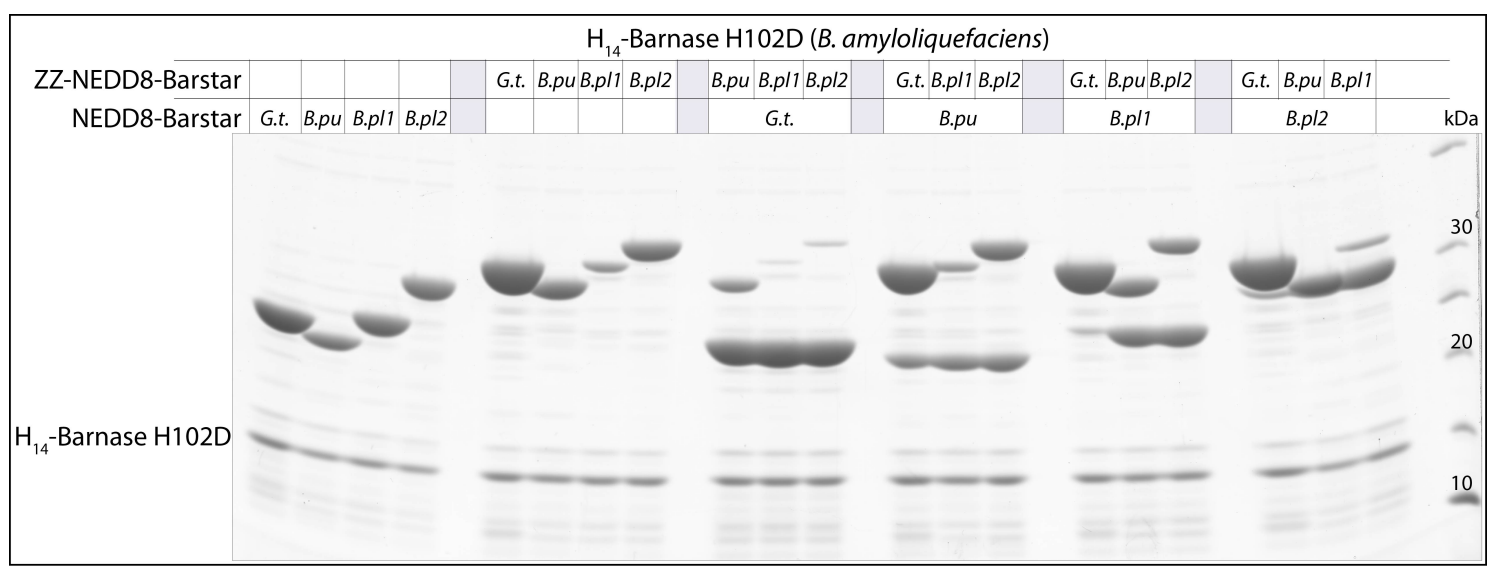

Figure 2.30: G.thermoglucosidasius Barstar strongly binds to B.amyloliquefaciens Barnase

E.coli lysate with $\mathrm{H}_{14}$-B.amyloliquefaciens Barnase $(\mathrm{H102 \textrm {D }})$ was incubated with lysates containing Barstar versions from various species tagged with either ZZ-brNEDD8-tag or ZZ-tag. The species used were: Geobacillus thermoglucosidasius (G.t.), B.pumilus (B.pu.), B.plantarum 1 (B.pl1.), and B.plantarum 2 (B.pl2.). The formed complexes were bound to $6 \% \mathrm{Ni}^{2+}$-chelate $500 \AA$ silica and eluted with SDS loading buffer. The Barstar versions were either incubated separately with the Barnase lysate or two Barstar versions were added to perform competition experiments. All samples were analyzed by SDS-PAGE using gradient polyacrylamide gels.

glucosidasius Barstar was complexed with three versions of the G.thermoglucosidasius Barnase and the B.amyloliquefaciens Barnase was complexed with the Barstar proteins from G.thermoglucosidasius, B.pumilus, and B.plantarum (consensus 1 and 2). All complexes, including the B.amyloliquefaciens complex, were analyzed by Thermofluor assays (for experimental details see 4.2.4.2). Small amounts of the protein samples are mixed with SyproOrange, a dye that binds to hydrophobic protein patches, and starts to fluoresce upon binding. The fluorescence is measured while the samples are applied to increasing temperatures (ranging from $30^{\circ} \mathrm{C}$ to $110^{\circ} \mathrm{C}$ ). The fluorescence increases upon protein denaturation and thus the resulting melting curves help to estimate the stability of a certain protein or protein complex.

Figure 2.31 shows the Thermofluor assay for the various Barnase:Barstar complexes. The previously analyzed B.amyloliquefaciens complex, depicted in blue, starts to denature at around $60^{\circ} \mathrm{C}$. Complexes containing either B.pumilus and B.plantarum Barstar proteins and the B.amyloliquefaciens Barnase seem to be less stable, as the melting temperature is lower (light green, cyan, dark green). All complexes containing at least one G.thermoglucosidasius component seem to be more stable as melting temperatures are all shifted to higher temperatures. The most stable complexes are G.thermoglucosidasius Barstar in complex with either G.thermoglucosidasiusBarnase ${ }_{(36-154)}$ (brown) or G.thermoglucosidasius Barnase $(32-154)$ (red).

This experiment together with the competition experiments and the solubility and expression assays strongly suggest that the G.thermoglucosidasius Barnase ${ }_{(36-154)}$ and the G.thermoglucosidasius Barstar are suitable to use as an affinity tag system. 




Figure 2.31: G.thermoglucosidasius Barnase:Barstar complexes are extremely stable

The following Barnase:Barstar complexes were formed and analyzed by Thermofluor: G.thermoglucosidasius $\operatorname{Barstar}_{(\mathrm{A50K})}$ bound to (1) G.thermoglucosidasius Barnase $(\mathrm{H108 \textrm {D }}, 42-154)$ (pink), (2) G.thermoglucosidasius Barnase $_{(H 108 \mathrm{D}, 36-154)}$ (brown), (3) G.thermoglucosidasius Barnase (H108D, 32-154) (red).

B.amyloliquefaciens Barnase (H102D) $_{\text {(1) }}$ bound to (4) B.amyloliquefaciens Barstar $_{(\mathrm{C} 42 \mathrm{~K})}$ (blue), (5) G.thermoglucosidasius $\operatorname{Barstar}_{(\mathrm{A} 50 \mathrm{~K})}$ (orange), (6) B.pumilus $\operatorname{Barstar}_{(\mathrm{C} 41 \mathrm{~K})}$ (dark green), (7) B.plantarum $1 \operatorname{Barstar}_{(\mathrm{C} 41 \mathrm{~K})}(\mathrm{cyan}),(8)$ B.plantarum $2 \operatorname{Barstar}_{(\mathrm{C} 41 \mathrm{~K})}$ (green)

\subsubsection{Structure determination of new Barnase:Barstar complexes}

Recently, the various complexes were used for crystallization trails, so far resulting in needle shaped crystals for the G.thermoglucosidasius Barnase $(36-154)$ :G.thermoglucosidasius Barstar complex (200mM Ammonium Sulphate, 100mM MES monohydrate pH6.5, 30\% PEG monomethylether $5000,20^{\circ} \mathrm{C}$, first crystals appeared 3 days after setting the drops) and the G.thermoglucosidasius Barnase ${ }_{(42-154)}$ :G.thermoglucosidasius Barstar complex (200mM Ammonium sulphate, 100mM Tris Sodium citrate dihydrate pH5.6, 25\% PEG 4000, $20^{\circ} \mathrm{C}$, first crystals appeared 3 days after setting the drops). In addition, small netlike crystals were obtained for the G.thermoglucosidasiusBarstar in complex with the B.amyloliquefaciens Barnase in the following conditions: 200mM Ammonium sulphate, 100mM Hepes pH7.5, 16\% PEG 4000, 10\% isopropanol, $20^{\circ} \mathrm{C}$, first crystals appeared 1 days after setting the drops.

However, the conditions need to be refined in order to obtain crystals that can be further analyzed. 


\subsubsection{Evolved Barnase:Barstar complex can be used as tag system for affinity chromatography}

The Barnase:Barstar system is a suitable pair for affinity chromatography. They bind with high affinity to each other, are both small, monomeric proteins and do not contain any disulfide bonds that might interfere with coupling to the MADA resin. The Barnase protein can be efficiently expressed and purified in E.coli. Barstar from B.amyloliquefaciens seems to have folding problems when expressed in E.coli, however, this problem was circumvented by switching to Barstar from the thermophil G.thermoglucosidasius.



Figure 2.32: Barnase:Barstar system can be used for affinity chromatography

(A): Barnase $\mathrm{H}_{102 \mathrm{D}}$-brSUMO-GFP was expressed in E.coli. The lysate (Input) was incubated with MADA beads to which Barstar ${ }_{\mathrm{C} 42 \mathrm{~K}}$ had been covalently attached. As control, empty beads were incubated with the lysate as well. In this experiment, $25 \mu \mathrm{l}$ MADA resin were incubated with either 200 or $500 \mu \mathrm{l}$ lysate. After binding for $1 \mathrm{~h}$, the flow through was collected (Flow through), the beads were washed and protease elution (0.1 $\mu$ M SENP1) was performed (Elution (protease)) resulting in a cleaved GFP protein. Samples were analyzed by SDS-PAGE using gradient polyacrylamide gels. (B) shows the same experiment. Pictures were taken of the Input samples (E.coli lysate containing expressed Barnase $\mathrm{H}_{102 \mathrm{D}}$-brSUMO-GFP), the beads after incubation, the beads after wash and after protease elution and the elution samples containing GFP only.

As proof of principle that a protein purification using the Barnase:Barstar pair is feasible, the Barstar protein (from B.amyloliquefaciens), was expressed in E.coli, purified by $\mathrm{Ni}^{2+}$-affinity chromatography and elution was performed by protease cleavage resulting in an $11 \mathrm{kDa}$ sized protein. Its C-terminal cystein was freshly reduced and the protein was covalently coupled to the MADA 2B Sepharose. This Barstar MADA resin was used as stationary phase during affinity chromatography. Next, an expression construct containing the B.amyloliquefaciens Barnase as N-terminal fusion tag was designed and cloned. As the Barnase sequence does not start with a methionine, an additional methionine was introduced to the very $\mathrm{N}$-terminus to enable translation initiation, resulting in an Met- 
Barnase-brSUMO-GFP construct. This construct was expressed in E.coli and the resulting lysate was used for affinity chromatography by mixing it with the Barstar MADA resin. After incubation for $1 \mathrm{~h}$ at $4^{\circ} \mathrm{C}$, the flow through was collected and the beads were washed with buffer. Subsequently, elution was performed using $0.1 \mu \mathrm{M}$ SENP1 protease and the cleaved protein was collected. All samples were analyzed by SDS-PAGE. In addition, the procedure was also documented by recording the fluorescence signal of GFP.

Figure 2.32 shows the first protein affinity chromatography purification using the Barnase:Barstar system. The input fraction in figure 2.32A shows the E.coli lysate containing the expressed Barnase-brSUMO-GFP construct. Two different amounts of lysate (200 and $500 \mu \mathrm{l}$ ) were incubated with empty beads (MADA 2B Sepharose only) and beads covalently linked to Barstar. The flow through samples still contain the expressed protein, indicating the beads were over-saturated. The protease elution samples contain the cleaved GFP protein $(\sim 30 \mathrm{kDa})$ but no a additional proteins. The empty beads do not show any eluted protein, once more proving the extremely low unspecific binding to the MADA resin. The low amounts of eluted protein from the resin might be caused by insufficient cleavage or by incomplete elution of the cleaved GFP product. Figure 2.32B shows the purification procedure step by step. The Input samples show a bright GFP signal. After incubation, the GFP signal is enriched on the Barstar MADA beads whereas no enrichment can be seen on the empty beads. This can also be observed after thorough washing. The protease cleavage leads to complete removal of the GFP from the beads, due to the high cleavage efficiency of the SENP1 protease. The GFP can be visualized in the protease elution fraction for the MADA Barstar beads.

It is noteworthy that Barnase can indeed be used as N-terminal tag and does not negatively influence the solubility or expression of the following fusion protein. However, this needs to be further analyzed also with more challenging fusion partners.

In summary, the tested Barnase:Barstar pair performs extremely well as affinity tag system and is - in many aspects - highly beneficial in comparison to the well-established Streptavidin:Biotin system. 


\section{Discussion}

\subsection{Analyzing the eukaryotic translation initiation apparatus}

Eukaryotic translation is a multistep process requiring the ribosome and 47 individual polypeptides, either acting as monomeric factors or as multisubunit complexes. In comparison, translation in bacteria requires only 9 individual proteins. One possible explanation for the drastic increase in complexity of the translation apparatus is the compartmentalization in eukaryotic cells. Gene expression is divided into two major processes: transcription and translation. In bacteria, these to processes are tightly coupled and thus most of the gene expression regulation happens at the level of transcription. In eukaryotes however, transcription and translation are spatially separated: DNA is transcribed to mRNA in the cell nucleus, the mRNA is exported to the cytoplasm where finally translation takes place. This separation requires and allows new mechanisms for gene expression also on the translational level, mainly during the initial step of translation, termed translation initiation. A strong argument for translation initiation being the most regulated and thus rate limiting step during eukaryotic translation is that out of the 47 proteins acting during this process, 37 operate during initiation.

Although protein synthesis is a major and absolutely fundamental step in all living cells, surprisingly less is known about the detailed interactions within the translation factor complexes or between different complexes in eukaryotes. Studies addressing these questions mainly focus on the yeast factors, which are often comprised of less subunits making them less complex. Another advantage when using yeast is that this model organism can be easily genetically modified and by use of temperature sensitive mutants, even mutations with lethal phenotypes can be introduced and analyzed.

In this study we aimed to shed light on the wheat translation initiation process by two main approaches: first, in vitro reconstitution of translation initiation factors, mainly eIF3; second, a wheat germ extract based translation assay to reveal the necessity of individual translation factor subunits and possible limitations in the system.

Studying the wheat apparatus is interesting and also advantageous for various reasons. First of all, wheat is a higher eukaryote with a complex and highly regulated translation system. Interestingly, plants do show differences in their translation apparatus composi- 
tion when compared to human or mouse, respectively. Plants have certain novel translation factors, e.g. the isoforms for eIF4F complex subunits eIF4isoE and eIF4isoG, or the so far only in plants identified nCBP (novel Cap Binding Protein). Next, for biochemical approaches, protein sequences from wheat are highly beneficial. The wheat species Triticum aestivum has a very broad temperature range, being able to survive under frost conditions but can also tolerate heat up to $50^{\circ} \mathrm{C}$. Thus, the proteins expressed in these species should have a stable and robust fold, which can be exploited during protein expression by screening over a wide range of expression conditions.

\subsubsection{In vitro reconstitution of the wheat elF3 complex}

For in vitro reconstitution of an eukaryotic multisubunit complex, the individual proteins are ideally expressed singly in E.coli, purified and assembled step by step in a test tube. However, this can be a highly challenging approach for a variety of reasons. Interactions within a complex happen mostly via hydrophobic interactions, meaning that the isolated subunits have one or more hydrophobic patches on their surface. These however, are solvent exposed when expressing the subunits individually, due to the fact that their natural binding partners normally shielding the hydrophobic areas are not present. Thus, these subunits are highly prone to aggregate, leading to the production of insoluble proteins. In addition, eukaryotic proteins might also depend on the eukaryotic chaperone system or specific factors needed for complex assembly that lack in E.coli.

In this study, we were able to express and purify all 13 subunits of the wheat eIF 3 complex after excessive screening of expression conditions and N-terminal fusion tags. In general, the amount of soluble protein increases when the proteins are expressed at lower temperatures and lower induction strength. When E.coli cells are exposed to lower temperatures, the entire cellular machinery slows down, potentially giving the proteins produced more time to fold properly prior to release from the ribosome. When additionally lowering the induction strength, less mRNA molecules are produced and transcribed, preventing partially folded proteins to accumulate, which would otherwise greatly increases the possibility of aggregation. Interestingly, the eIF3 subunits that perform well also with higher induction strength and slightly higher expression temperatures, such as eIF3b, eIF3c and eIF3d are among the largest eIF3 subunits with molecular weights from $65 \mathrm{kDa}$ to $105 \mathrm{kDa}$. When applying the sequences to secondary structure predictions, these proteins contain distinct domains with known stable folds, e.g. PCI- or WD40-domains. Perhaps, these domains fold quick enough to shield the remaining emerging polypeptide chain towards the solvent, whereas smaller proteins with less defined domains and partially disordered regions can not act in a self-supporting manner.

This shielding effect can also be mimiced by large N-terminal fusion tags. All 13 eIF3 
subunits were analyzed for their expression and solubility when fused to eight different N-terminal fusion tags (see table 2.1). For some subunits (eIF3b, eIF3c, eIF3d, eIF3j and eIF3k), the N-terminal fusion tag only marginally affects the proteins expression level and solubility. The remaining eIF3 subunits tend to be insoluble when expressed with small $\mathrm{N}$-terminal fusion tags such as $\mathrm{H}_{14^{-}}$or $\mathrm{H}_{14}$-TEV-, but the solubility increases with increasing tag size. The two most problematic eIF3 subunits are eIF3a and eIF31. These two subunits can only be solubly expressed when fused to $\mathrm{H}_{14}$-TEV-MBP $/ \mathrm{H}_{14}$-brSUMO-MBP but remain insoluble when fused to GFP, which is similar in size. MBP (maltose binding protein) was previously described to have chaperone - like qualities, utilizing its hydrophobic cleft as an ideal folding environment for the emerging polypeptide chain (Pryor and Leiting, 1997; Smyth et al., 2003). Hence, MBP is not only large enough to shield an emerging polypeptide towards the solvent, it also positively influences the folding of the newly synthesized protein.

Assuming that the model of subunit arrangement for the human eIF3 complex published by Zhou et al. (2008) and Querol-Audi et al. (2013) (see figures 1.2 and 1.3) is similar to the wheat eIF3 complex, it is surprising that subunits buried inside the complex seem to have the least solubility problems, such as eIF3c respectively. It brings together three eIF3 subcomplexes and consequently should have a highly hydrophobic surface. But as previously mentioned, eIF3c can be expressed to high amounts with small tags even at elevated temperatures (see panel 2 in figure 2.1). In comparison, subunits eIF3a and eIF31 seem to be rather peripheral and hence should have surface properties tolerating solvent exposure. However, we can not rule out that additional factors in the wheat system promote the proper folding or assembly of these proteins. During synthesis in E.coli these factors are not available and thus folding might be problematic.

All 13 subunits could be further purified by affinity chromatography and protease elution resulting in tag-free proteins. Interestingly, even the proteins showing low solubility do not precipitate upon cleavage, indicating a stable fold that might be supported by the $\mathrm{N}$-terminal tag but once formed is stable on its own. However, when performing binding assays with the purified proteins, no interactions could be observed. Technical issues were excluded by performing control experiments in parallel showing the interaction between the nuclear transport factor Importin $\beta$ and the IBB domain of Importin $\alpha$ (Görlich et al., 1995). Binding assays with the eIF3 subunits were performed pairwise but also with mixtures resembling the sub-complexes described by Phan et al. (1998) and Zhou et al. (2008) to exclude the possibility that a pairwise interaction might be too weak and the subunits bind in a corporative manner. In addition, experiments were also performed in the presence of wheat germ extract to provide possible further subunits or assembly factors missing in the E.coli system. 
Failing to reconstitute the eIF3 complex or at least sub-complexes in vitro, we were interested in the stability and composition of the native wheat eIF3 complex. Possibly, the complex itself is very fragile, assembling only upon requirement or presence of other translation factors such as eIF2. However, wheat germ extract fractionation by gelfiltration and IP experiments revealed the presence of a stable pre-assembled complex in the native system only lacking the loosely attached eIF3j subunit. In addition, the high abundant elongation factor eEF1 $\alpha$ co-precipitated with the eIF3 complex. Further factors were not identified, suggesting that no additional factors are stably attached to the eIF3 complex in vivo. Possible assembly factors only transiently interacting with the complex can not be identified by IP experiments but can be addressed by cross-linking experiments in the future.

Besides the hypothesis that in vitro binding of recombinantly produced eIF3 subunits does not occur due to a lack of essential factors, another explanation might be that although the proteins can be expressed in a soluble manner, the folding is not correct resulting in molten globules that are non- or misfunctional. When purifying eIF3b from E.coli lysate, the bacterial chaperone GroEL co-purifies and seems to form a stable complex with the eIF3 subunit as they also co-migrate through a gel filtration column (data not shown). The presence of a bacterial chaperones strongly argues for folding problems of the eukaryotic protein in a bacterial environment. eIF3 subunits are also heavily posttranslationally modified. These modifications might be essential for subunit interaction and can not be introduced when the proteins are expressed in E.coli.

As previously mentioned, the lack of the eukaryotic chaperone system in bacteria in general is an immense burden for all eukaryotic proteins expressed in E.coli. The main cytoplasmic eukaryotic chaperone is the TRiC complex, first identified in 1990 (Gupta, 1990). TRiC is composed of two rings of eight different but related subunits and shows an intrinsic ATPase activity. In addition, prefoldin, another heterohexameric chaperone functions together with the TRiC complex to promote proper folding of produced proteins, mainly cytoskeletal proteins such as actin or tubulin. Stemp et al. (2005) showed that actin expressed in E.coli can be converted to a soluble protein when the TRiC complex (purified from HeLa cells) was added to the lysate. This strategy could be used to obtain properly folded eIF3 subunits but could be even further exploited, e.g. by co-expressing the TRiC complex in bacteria. This is indeed a challenging approach but would be highly beneficial for the expression of eukaryotic proteins in a bacterial expression system in general. 


\subsubsection{In vitro wheat germ translation assay as tool to study effects of translation (initiation) factors}

Another approach to study the eukaryotic translation apparatus is to look at an in vitro translation assay. The system can be manipulated, e.g. by depletion of certain translation factors, thereby validating their necessity for overall translation. It is important to mention that in such a system only the effect on translation is monitored. This enables to introduce manipulations that would immediately cause lethality in an in vivo system. The assay used in this study is based on the in-house produced wheat germ extract, which is supplemented with the mRNA of the reporter construct (Firefly Luciferase), amino acids and an energy regenerating system. The translation efficiency of the extract can then be validated by the amount of produced luciferase, measured by a luminescence assay.

The manipulation was performed by either depleting certain translation factors or translation factor complexes by subunit specific antibodies or by adding recombinantly expressed and purified translation factors to the extract. In case of further addition of translation factors, no significant changes in overall translation efficiencies were observed compared to non-supplemented extracts. This might have several reasons: first of all we do not know the limitations of the system. Dr. Enke showed that the wheat germ extract produced in-house shows an 8-fold better translation efficiency than commercial available extracts (Promega; Enke, 2010), indicating an extremely high quality of the system. Addition of certain translation factors would only show a positive effect, when the factor is limiting in the system, however, this does not seem to be the case. It remains to be elucidated, if the added recombinant translation factors are correctly folded and thus functional and if lacking posttranslational modifications are required for proper function. Additionally, the timescale of cleavage of the added $\mathrm{H}_{14}$-MBP-brSUMO fusion constructs needs to be monitored. If cleavage requires too much time, formation of multisubunit complexes might have already occurred without the incorporation of the recombinant factors.

Depletion of translation factors however, resulted in a decreased translation efficiency in most of the cases (see figure 2.6). After depleting the wheat germ extract using eIF subunit specific antibodies, the extracts were analyzed by Western Blot. When using antibodies recognizing the large eIF3 subunits (eIF3a, eIF3b, eIF3c and eIF3d), all other eIF3 subunits (except eIF3j) co-precipitate, once again strongly arguing for eIF3 being present as a stable complex in the wheat germ extract. However, depletion is still incomplete probably caused by capacity effects or by shielded epitops as soon as the subunits are incorporated into the complex. Future depletions might be performed such, that the extract is applied in consecutive rounds to beads coupled to antibodies against different eIF3 subunits causing an avidity effect.

Interestingly, although eIF3 is still present in the extract, overall translation rates decrease 
$\sim 2$-fold. As eIF3 is a multiplayer during translation initiation, even small changes in eIF3 concentration could reduce the efficiency of protein synthesis, e.g. by requiring more time for proper 43S PIC assembly or recruitment of the mRNA to the 40S ribosomal subunit. In addition, lower amounts of eIF3 in the system could also affect the initiation fidelity and translation could start at non-AUG codons. Ribosome profiling experiments could reveal, if ribosomes accumulate at alternative start-codons upon reduction of eIF3 in the wheat germ extract.

The strongest effect however, with an approximate translation efficiency decrease of 8fold, was observed when depleting the extract with eIF5 specific antibodies. Western Blot analysis revealed an almost complete removal of endogenous protein. eIF5 is a GTPase activating protein (GAP), hydrolyzing GTP of the eIF2 complex upon codon-anticodon pairing (reviewed in Marintchev and Wagner, 2004). If eIF5 gets limiting in the extract, hydrolysis in eIF2 is impaired, causing problems in correct and stable binding of the initiator tRNA to the P-site of the ribosome. A strong reduction in translation rates also occurs when eEF1B $\beta$ levels are reduced. The subunit is part of the GEF eEF1B, acting on eEF1A. If the GEF is non-functional due to the lack of one of its subunits, GDP exchange to GTP in eEF1A is impaired, preventing the elongation factor to participate in a new round of translation elongation.

However, although preliminary answers to the question, which translation factors/factor subunits are required for proper translation could be obtained by using the wheat germ in vitro translation assay, it is extremely difficult to obtain conclusive and trustworthy data. The wheat germ extract itself is highly sensitive towards even minimal changes in the buffer composition, making it nearly impossible to reproduce experiments. In addition, each newly produced wheat germ extract batch must be screened for its own optimal salt conditions and even if extracts with their optimal salt composition are compared, the values for the amount of produced Firefly luciferase varies greatly. It is difficult to hypothesize why these extracts are so sensitive, and by what means the robustness could be increased. In general, working with undefined systems containing vast amounts of different proteins, lipids and small molecules entails the risk of undefined side reactions that might lead to misinterpretation of the obtained data. Alternatively, an in vitro reconstituted translation system would be an ideal basis for analysis of individual components. Alkalaeva et al. (2006) introduced a human in vitro translation system reconstituted from purified ribosomal subunits, initiation, elongation and termination factors and aminoacyl tRNAs (purified from HeLa lysate). The effects of certain translation factors can be analyzed by excluding the component of interest in the reconstitution mix. However, also this system has limitations such that only entire complexes can be analyzed. A reconstituted eukaryotic system using only recombinantly expressed and purified translation factors 
would allow to study the effect of single subunits or even more in detail what happens, when certain truncated or mutated proteins are added to the system. This challenging but groundbreaking technique would finally allow to study the highly complex eukaryotic translation apparatus in depth.

\subsection{Nuclear transport factors might act as compartment-specific inhibitors of translation}

In eukaryotic cells the processes of transcription and translation are spatially separated: transcription happens in the nuclear interior whereas translation occurs in the cytoplasm. Nevertheless, there are reports suggesting translation also taking place in the cell nucleus (Goidl et al., 1975; Iborra et al., 2001), although this would have dramatic effects on overall cell viability and is under strong debate. Most mRNA molecules are spliced in the nuclear interior prior to transport to the cytoplasm. During splicing, the intron regions are removed resulting in the final ORF of the protein to be translated. If mRNAs were translated prior to splicing, the possibility of creating truncated protein products would be extremely high as intron regions often harbor premature stop codons. These truncated versions might cause dominant negative effects in the cell. Further disadvantages of nuclear translation would be that an important level of regulation would be abolished and certain proteins that could have negative influence on gene expression might mislocalize to the nucleus.

Eukaryotic cells evolved many mechanisms to avoid translation occurring in the nuclear interior. Ribosomes, although partly pre-assembled in the nucleus, gain their final maturation only in the cytoplasm and the concentration of translation factors in the nuclear interior is actively kept low. Mislocalized or freely diffusing translation factors are shuttled back to the cytoplasm by active transport via nuclear export factors. The nuclear export factor Exportin 4 is known to transport the small translation factor eIF5A back to the cytoplasm (Lipowsky et al., 2000), eEF1A in complex with tRNAs is exported from the nucleus by Exportin 5 and eIF $2 \beta$ is removed from the nucleus by Crm1 (Bohnsack et al., 2002). For Crm1 it was additionally shown that it binds a wide range of different translation factors (unpublished data).

These findings lead us to the hypothesis that nuclear transport factors might act as compartment specific inhibitors of translation, thereby contributing another argument to weaken the hypothesis of nuclear translation. 


\subsubsection{Depletion or sequestration of elF5A has only subtle effects on the translation of poly proline containing reporters}

Recent studies suggest that yeast eIF5A and its bacterial homologue EF-P play a role in the translation of mRNAs coding for poly-proline stretches (Doerfel et al., 2013; Gutierrez et al., 2013). In bacteria, the ribosome stalls upon incorporation of at least two consecutive prolines to the emerging polypeptide chain and yet another incoming proline or glycine residue. The deacetylated tRNA leaves the E-site and due to stalling this site is not further occupied by the next tRNA. EF-P binds to the ribosome and accommodates between the E- and the P-site, promoting peptide bond formation of ProPro or ProGly pairs (Doerfel and Rodnina, 2013). In the absence of EF-P, stalling can not be resumed and a full length proline rich protein can not be synthesized.

eIF5A is a direct transport cargo of the nuclear export factor Exportin 4, efficiently removing this small translation factor out of the nucleus in a Ran dependent manner. To test the hypothesis whether the presence of a nuclear transport factor can indeed interfere with translation, a rabbit reticulocyte lysate in vitro translation assay was performed using a poly-proline and a poly-serine containing construct as reporters (see figure 2.8). The lysate was supplemented with recombinantly expressed and purified Exportin 4 and Ran. According to findings in bacteria, clusters of ProProPro or ProProGly are sufficient to stall the ribosome on the mRNA transcript. No full length protein can be produced in case EF-P is missing. In yeast, ribosome stalling occurs in the absence of fully functional eIF5A on poly-proline motives with the second or third proline sitting on the P-site of the ribosome (Gutierrez et al., 2013). The poly-proline reporter construct used in this study contains one poly-proline cluster with twelve consecutive prolines and thus should be sufficient to cause an immediate stalling of the ribosome on the transcript as soon as eIF5A is sequestered. When supplementing an in vitro translation assay with Exportin 4 and Ran, the poly-proline reporter is indeed translated less efficiently compared to either the poly-serine reporter or translation in a non-supplemented extract. The effect however is very subtle possibly indicating rather a pausing of the ribosome and thus a delay in translation rather than a complete stalling (see figure 2.12). Interestingly, when translating other reporters, namely the Firefly luciferase containing 29 prolines or the two yeast proteins Ldb17 (23 prolines) and Eap1 (72 prolines), the effect upon Exportin 4 and Ran addition is strongly enhanced. Note that in general the decrease in translation efficiency can only be observed, when Exportin4 and Ran are added. Supplementing the extract with Exportin4 alone does not effect the translation rates, strongly suggesting that the binding and thus sequestration of eIF5A by Exportin 4 happens in a Ran dependent manner as expected for a nuclear export factor.

Studies showing that eIF5A is involved in the translation of poly-proline containing pro- 
teins were so far only performed in yeast, a simple eukaryote. Nevertheless, the observation that a cluster of twelve consecutive prolines does not show a very strong effect in a translation system derived from higher eukaryotes was rather surprising. When briefly comparing the translation apparatus of yeast to the process in plants or mammals, it is obvious that the process increased dramatically in complexity and thus effects shown in yeast might be hints that factors function in a similar manner also in other eukaryotes, but the results are not directly transferable. Specific sequence characteristics causing the ribosome to pause or to stall on the mRNA in higher eukaryotes could potentially be identified by two experimental approaches: 1) reporter constructs with different proline cluster lengths or clusters combining prolines with other amino acids could be analyzed in the presence or absence of eIF5A (depleted or sequestered). 2) A rather unbiased approach could be to purify endogenous mRNA from tissue and translate this mRNA pool in an in vitro translation assay with and without eIF5A activity. Upon comparison of the translated proteins, changes in translation rates of certain proteins could be identified and the underlying amino acid sequence could be analyzed.

In conclusion, we were able to show that by sequestering eIF5A by its nuclear export factor Exportin 4, the translation efficiency of reporter constructs rich in prolines decreased. Nonetheless, add-back experiments with recombinantly expressed, purified and in vitro hypusinated eIF5A need to be performed. However, it is the first hint that eIF5A also in higher eukaryotes is required for proper synthesis of proline rich proteins. Its putative role in peptide bond formation between consecutive prolines in general might raise the discussion if eIF5A is an initiation factor, as implied by the name, or if its actual function is taking place during translation elongation.

\subsubsection{Addition of Crm1 to an in vitro translation assay decreases translation efficiency dramatically}

Due to the wide cargo range of Crm1 and the finding that this nuclear export factor can pull out almost all translation (initiation) factors from a HeLa cell lysate in a Ran dependent manner (unpublished data by K. Kirli), we wanted to analyze the effect upon Crm1 addition to an in vitro translation assay. In comparison to Exportin 4, that only interacts with specific cargo proteins and thus the reporter constructs needed to fulfill certain requirements, translation rates of a variety of different reporters should be affected upon Crm1 addition. Here, the poly-serine, poly-proline and Firefly luciferase reporters were used.

When adding recombinantly expressed and purified Crm1 WT, the reporter constructs are translated slightly less efficient as compared to a non-supplemented extract. However, by additionally supplementing the extract with Ran (in 3-fold excess over Crm1), the trans- 
lation efficiency drops significantly so that no reporter signal can be observed any longer. The same holds true when adding $\mathrm{Crm} 1_{\mathrm{G} 502 \mathrm{~A}}$, a Ran independent $\mathrm{Crm} 1$ variant. The effect seems to be concentration dependent, as it can only be observed when adding $>3 \mu \mathrm{M}$ Crm1. When supplementing the in vitro translation assay with other nuclear transport factors such as Exportin 6, mediating actin export (Stüven et al., 2003) or CAS, responsible for recycling Importin $\alpha$ to the cytoplasm (Kutay et al., 1997), the effect can not be observed, neither in absence nor presence of Ran (see figure 2.12). These observations argue that the decrease in translation is related to the addition of nuclear transport factors indeed being known to interact with translation factors.

However, it is unlikely to assume, that Crm1 is able to bind each single subunit directly. When analyzing the translation factors pulled out from HeLa cell lysate by Crm1, only a minor portion harbors a sequence stretch that has the properties to act as a nuclear export signal (NES). Interestingly, each multisubunit complex contains at least one subunit with a predicted NES, which seems to be enough for co-purification of the entire complex in the pull-out assay. But what happens in the in vitro translation assay?

If one assumes that certain translation factors or translation factor subunits bind to Crm1 via their NES, they most likely are sequestered because important interaction sites are shielded by Crm1. Another explanation might be that even if a functional site of a translation factor would still be exposed, the Crm1:translation factor complex could simply be too large to act during translation (initiation). Crm1 might also promote a platforming effect, meaning that once a translation factor is bound, further interacting factors assemble on to it. Thereby more and more translation factors are taken out of the actual translation process and translation efficiency drops. The observed slight decrease when adding Crm1 only might be explained by some non-Ran mediated binding of translation factors to Crm1.

So far, we hypothesize that binding and thus sequestration of the translation factors to Crm1 happens by NES binding to the hydrophobic cleft of Crm1. Hence, blocking or mutating the hydrophobic cleft should be able to reverse the decreased translation efficiency. The hydrophobic cleft was blocked by either supplementing the extract with NS2 peptide, which shows high affinity towards Crm1s hydrophobic cleft (Engelsma et al., 2008), or Snurportin1 fused to either PKI-NES or REV-NES to further strengthen the interaction between Crm1 and its natural cargo. In addition, the translation assays were performed with mutant versions of Crm1, which at least in vitro are not able to bind a PKI peptide any longer (see figure 2.13). Surprisingly, the observed decrease of translation efficiency upon Crm1 and Ran addition could not be reversed by any of the applied approaches.

There are a variety of possible explanations why the observed effect could not be reversed so far. Although it is known that the NS2 peptide can bind Crm1 strongly in an in vitro 
binding assay, the conditions in a lysate are very different. Not only that the peptide could easily be degraded by proteases in the extract, there might in addition be structures in the lysate to which the NS2 peptide is attracted. Thus, we can not ensure that the NS2 peptide reaches and binds Crm1, thereby blocking the hydrophobic cleft or competing out translation factors. Even if it does reach Crm1, the affinity of the NS2 peptide towards Crm1 might not be strong enough to displace pre-bound cargos.

Next, the PKI-Snurportin or REV-Snurportin proteins were added with the aim to block the hydrophobic cleft of Crm1 in the extract. The first Crm1 crystal structure was obtained in complex with Ran and Snurportin1, a natural Crm1 cargo described in 1999 (Paraskeva et al., 1999; Mönecke et al., 2009). Interestingly, the interaction between Crm1 and Snurportin1 is not exclusively via the hydrophobic cleft but Snurportin1 also interacts with several other residues on Crm1s HEAT-repeats 12-14, thereby shielding a considerable portion of Crm1s surface, making it inaccessible for other cargoes. Snurportin1 was used as fusion protein to either PKI-NES or PEV-NES to further increase the binding affinities. Surprisingly, also supplementing the in vitro translation assay with these proteins did not lead to a rescue of translation efficiency. However, Crm1 was not pre-complexed with the Snurportin1 fusion proteins before addition to the extract. Thus, the problems of accessibility and competition for binding in the lysate still remains. As already described, addition of Crm1 hydrophobic cleft mutants $\left(\mathrm{Crm}_{\mathrm{A} 451 \mathrm{R}}, \mathrm{Crm} 1_{\mathrm{K} 568 \mathrm{E}}\right)$ which can no longer interact with PKI-NES peptides in vitro (unpublished data, H. Chug), also led to a significant decrease in translation efficiency of the reporter constructs. Yet another approach to block Crm1 towards NES binding is to use the toxin leptomycin B that specifically binds to the hydrophobic cleft, thereby covalently modifying a cysteine residue (Nishi et al., 1994; Kudo et al., 1998). However, leptomycin B can not be solubelized to high concentrations needed to efficiently block Crm1 in the lysate.

When looking at Exportin 4, the decrease in translation efficiency is - as expected - only observed on poly-proline containing proteins, which once again claims that eIF5A, the cargo of Exportin 4 plays a role in the translation of poly-proline proteins. In case of Crm1 however, a broad range of cargoes can be recognized and the binding mode of nuclear export factor and cargo is different to the binding mode of other NTRs. Most nuclear export factors bind their cargos by wrapping around them; the cargo interacts mainly with the inner surface of the transport receptor. Crm1 however, has in comparison a rather small interacting surface with the cargo which is also located more at the outer side of the HEAT repeats. The interaction between the NES and the hydrophobic cleft of Crm1 is based on a short, hydrophobic amino acid stretch. Also more and more non-classical NES motives are identified. Taking together, Crm1 seems to have less stringent modes of binding towards its cargo, thus the binding and sequestration of Crm1 towards the 
translation factors must not necessarily happen via the hydrophobic cleft only, explaining why even in presence of hydrophobic cleft mutants, Crm1 seems to be able to efficiently block translation. One can nevertheless also argue that the effect might be unspecific, caused by a folding stress mechanism acting on translation and triggered upon addition of possibly misfolded eukaryotic proteins expressed in E.coli. This would explain, why the effect can not be reversed by specifically blocking the interaction between Crm1 and its cargos. Other findings, namely the decrease of translation rates in a Ran dependent manner and the possibility to add other nuclear transport factors expressed in E.coli to the extract without affecting translation rates, however do suggest that Crm1 (or Exportin 4) are specifically provoking the observed translation efficiency decrease.

\subsubsection{Is nuclear translation possible, if NTRs interfere with translation?}

The hypothesis of nuclear translation appeared the first time in 1954, showing that radiolabeled amino acids can be rapidly incorporated in nuclear proteins (Allfrey, 1954). Another major experiment trying to validate the hypothesis was performed by Goidl et al. (1975), showing that polyribosomes can be isolated from nuclei. Nowadays, the hypothesis builds on two main arguments: (1) nuclei contain all necessary components for translation (e.g. Lejbkowicz et al., 1992), (2) isolated nuclei can aminoacetylate tRNAs and incorporate radio labeled amino acids into proteins (Lund and Dahlberg, 1998). However, both arguments can be easily refuted because even if all proteins required for translation would be present in the nucleus, it is not proven that these are also fully active. Ribosomes for example maturate in the cytoplasm and no re-import of fully mature and assembled ribosomes to the nucleus is known. The second argument is in parts even questioned by supporters of the nuclear translation theory themselves, stating that these effects might possibly also be caused by "contaminating cytoplasmic machinery on the outer nuclear membrane" (Iborra et al., 2001). The finding of "newly synthesized proteins" in the nucleus labeled by heavy amino acids is more likely to be caused by rapid import of proteins from the cytoplasm to the nuclear interior (Ribbeck and Görlich, 2001). So far, the location of synthesis was not addressed.

As previously mentioned, protein synthesis in the nuclear interior would have dramatic negative impact on the cell. Truncated protein products could be synthesized due to translation of non-or misspliced mRNAs. Further, proteins interfering with proper gene expression could be mislocalized to the nucleus. The cells active mechanisms to remove mislocalized translation factors from the nucleus back to the cytoplasm is one of the strongest arguments for us that translation in the nucleus is unlikely to occur. The findings that nuclear transport factors, which are known to bind to translation factors can indeed interfere with translation in an in vitro translation system strongly indicates that 
nuclear transport factors might play a dual role in this context: shuttling out the translation factors from the nuclear interior and - during binding in the nuclear interior - active sequestration of the translation factor function.

\subsection{Novel tools for protein purification: a MADA activated resin and Barnase:Barstar as high affinity pair}

Affinity chromatography is a widespread tool for efficient protein purification, based on the interaction between two molecules. These can be either two proteins, e.g. antibodies and their corresponding antigen, or a protein/peptide chelating a metal ion (e.g. a polyhistidine peptide recognizing $\mathrm{Ni}^{2+}$ ions) or recognizing a biochemical modification as it is the case for streptavidin and biotinylated proteins. One member of the affinity pair is usually immobilized on a solid phase (silica, Sepharose or magnetic beads), the other one is added to the desired protein as fusion tag or chemical modification.

In this study, we developed and characterized not only a novel and profitable procedure to immobilize proteins to a solid phase, but also a new affinity pair, which has major advantages to other commercial available tag systems.

\subsubsection{MADA activated resins as tailor-made supports for a broad range of applications}

An efficient way to covalently attach proteins to a stationary phase is by forming a thioether bond between an activation group on the resin and the sulfhydryl group of an exposed and reduced cysteine on the protein to be immobilized. SulfoLink (Thermo Scientific) is the most commonly used commercial available resin containing sulfhydrylreactive chemistry. It is based on iodoacetamide groups that form stable thioether bonds preferably at physiologic or alkaline conditions. Interestingly, a long, hydrophobic spacer is introduced between the resin and the active group, which can be disadvantageous for downstream experiments, e.g. by causing unspecific binding of proteins. In addition, haloacetamide derivatives predominantly react with cysteine side chains, but also other residues such as tyrosines, histidines and tryptophan can be modified.

Another possibility to activate resins to react with thiol groups is using maleimide chemistry. The reaction occurs at almost neutral $\mathrm{pH}$ conditions and the formation of a covalent thioether bond does not result in any side products. Surprisingly, the chemistry is widespread for labeling of proteins with biotin or fluorophores but is not routinely used for immobilization of proteins to beads, although it is very straight forward and requires mainly cost-efficient compounds during production.

The MADA 2B Sepharose characterized in this study is activated by MADA (a propri- 
etary chemistry on beads developed in our lab) that reacts with the sulfhydryl group of the cysteins (see figure 2.14). In addition, a Tris moiety is introduced to the resin as well, providing an overall hydrophilicity of the resin. This moiety can also be exchanged for charges or weak hydrophobicity, according to the needs of the respective application. As expected, the resin is highly specific towards reduced cysteins, enabling to couple a variety of different ligands such as ProteinA, the ZZ domain-recognizing affibody ZpA963, streptavidin or designed binding constructs as depicted in figure 2.20A. Ideally, the coupled proteins contain only one exposed cysteine to enable directed immobilization. Hence, cysteine residues were introduced to the extreme C-terminus of the proteins after a short glycine-serine rich spacer, enabling a defined distance between the resin and the folded structures of the protein.

Efficient coupling was observed in presence of the main standard buffer systems at neutral and slightly alkaline conditions $(\mathrm{pH} 6.5-8.5)$ and at temperatures from $4^{\circ} \mathrm{C}$ to $37^{\circ} \mathrm{C}$. Coupling is prevented in presence of the reducing agents DTT or TCEP but thioether formation occurs in presence of $\mathrm{NaBH}_{4}$ and the routinely used bacteriostatic $\mathrm{NaN}_{3}$.

The MADA activated 2B Sepharose or magnetic beads can be used for a broad range of biochemical applications. (1) Affinity chromatography (2) Pull-down assays (3) Immunoprecipitation experiments and (4) Purification of large protein complexes from a lysate. In all applications tested, an extremely low background binding was observed and in comparison to commercially available affinity resins, the MADA resin was either as efficient as the commercial products or even performed superior in terms of background binding or capacity.

The easy and cheap production of the MADA resin and its modularity is highly beneficial. In principle, it allows every laboratory to create an affinity resin tailor-made for their respective application without the need to compromise. The chemistry can be applied to all common resins, such as Sepharose, silica or magnetic beads. Sepharose and silica resins are mainly used for protein purification purposes but also small scale interaction studies. Magnetic beads however are not used for protein purification as the yield is in the $\mu \mathrm{g}$ range. But this support is commonly used in interaction studies and for highthroughput screenings such as phage display, respectively. Depending on the application, the coupling density of the affinity protein to the MADA resin can also be individually adjusted. The larger the complexes or structures are that should be pulled out from a lysate, the lower the coupling density of the resin should be to prevent steric hinderance or clashes of the complexes of interest. As coupling is performed in-house, these parameters can be influences, whereas this is not the case for commercial available systems that tend to have a rather high coupling density for a maximal yield of "normal sized" proteins. To further decrease the probability of steric hinderance on the resin, the spacer introduced 
to the affinity protein between the C-terminal cysteine and the folded domain structure can be elongated. As mentioned previously, the MADA resin used in this study contains a hydrophilic Tris moiety. Depending on the application, this hydrophilic component can be exchanged to charged or hydrophobic moieties. For basic proteins, e.g. histones or ribosomal proteins, a slightly positive charged resin could be beneficial to avoid ion exchange effect that might interfere with proper elution of the protein from the resin.

Depending on the application, various ligands can be immobilized to the resin. Most common are streptavidin, ProteinA/ZZ-domain, or the affibody ZpA963 recognizing ZZtagged proteins. Besides these obvious affinity proteins, binding proteins can be designed for a specific purpose (see figure 2.20). Here, the construct enables to perform immunoprecipitation experiments, where the pulled out complexes are not eluted by acidic elution but can be eluted in native buffer by protease cleavage. This is a novel technique to purify large protein complexes or even cellular structures by affinity chromatography without utilizing multistep protocols including different density centrifugation cycles, respectively. Another binding construct could make use of the Importin $\beta$ mutant fused to a protease cleavage site and the C-terminal cysteine. Coupled to MADA magnetic beads, the resin should be able to specifically pull out nuclear pore components or even annulate lamellae from a lysate as the Importin $\beta$ mutant is known to bind nucleoporins (Kutay et al., 1997).

\subsubsection{Barnase:Barstar affinity tag system}

Affinity chromatography is based on a pair of molecules that ideally strongly bind to each other in a highly specific manner. One of the most prominent affinity pairs is streptavidin binding to the small molecule biotin. Streptavidin is a $16 \mathrm{kDa}$ protein produced by the bacteria Streptomyces avidinii and was first described by Tausig and Wolf (1964). It binds biotinylated proteins with extremely high affinity of $\mathrm{K}_{\mathrm{D}}=10^{-14} \mathrm{M}$ (Green, 1990) and once formed the bond can resist broad ranges of $\mathrm{pH}$, temperatures, organic solvents or denaturing compounds. However, besides these advantageous features, the affinity pair also has severe disadvantages. (1) Biotin is present in all living cells at least in small amounts. Hence, whenever using streptavidin resins to extract proteins from a lysate, endogenous biotin will cause background binding, which might interfere with proper evaluation of resulting data. (2) Streptavidin itself is rather small, but it forms a homotetramer with a size of around $60 \mathrm{kDa}$. Coupling the tetramer to the resin causes two major problems: either the tetramer is attached by four streptavidin subunits building up a meshwork which might interfere with binding and accessibility of large molecules to the resin, or, if a tetramer is attached by fewer than four subunits, a leakage of streptavidin from the matrix is possible. (3) Streptavidin itself is difficult to express and to purify in large amounts from E.coli. Even shortened versions, that are reported to show a higher level of 
expression as the wild type (Sano et al., 1995), have severe solubility problems. (4) Streptavidin recognizes biotinylated proteins, a modification, that is not efficiently introduced to proteins containing a biotinylation site when expressed in E.coli. Thus, the protein has to be either co-expressed with the biotinylation enzyme BirA, or a chemical or enzymatic modification has to be performed in vitro after purification. In both cases, the production of biotinylated proteins that can be recognized by streptavidin, requires additional hands on time as compared to simply expressing a fusion protein.

The extracellular RNase Barnase and its intracellular inhibitor Barstar show advantages over the streptavidin:biotin affinity pair in all four aspects. The pair is formed by two polypeptide chains, thus a target protein must be fused to either Barstar or Barnase but no additional modification is required. The small size of the proteins and their monomeric nature enable the direct coupling of defined amounts to a stationary phase without the risk of forming an intertwined layer that is prone to interfere with proper target protein binding or leakage of non covalently bound subunits. The strong interaction between Barnase and Barstar $\left(K_{D}=10^{-14}\right.$, Schreiber and Fersht, 1993) that even tolerates ionic strengths of $\sim 5 \mathrm{M} \mathrm{NaCl}$, prevents leakage of the fused target protein once bound to the affinity partner. This however requires elution of the target protein by protease cleavage and not by any means of competition as it is the case for His-tagged proteins and imidazole, respectively. Elution by protease cleavage is highly specific. Only proteins containing the protease recognition site are efficiently cleaved off the resin whereas proteins unspecifically binding the resin remain unaffected. This is beneficial in sample preparation, mainly for subsequent experiments that either depend on sensitive detection such as mass spectrometry or require highly homogenous samples, e.g. dynamic light scattering or protein crystallization. In addition, also unspecific binding of endogenous proteins to the immobilized affinity protein is very low as the Barnase:Barstar interaction is highly specific. Barstars only function in the bacterial cell is to bind and thus sequester the enzymatic activity of barnase. Barnase however functions extracellularly.

As previously described, it must be possible to efficiently express and purify both proteins of the affinity pair also in absence of the binding partner. Barnase, which hydrolyzes ribonucleotides by an acid-base mechanisms using residues $\mathrm{Glu}_{73}$ and His $\mathrm{H}_{102}$ (Mossakowska et al., 1989), causes lethality when expressed in E.coli. Hence, the active center was mutated by replacing $\mathrm{His}_{102}$ with aspartic acid. The introduced negative charge prevents the catalytic mechanism and barnase can be expressed to high levels in E.coli. Although the wild type barstar protein is able to bind the mutated barnase protein (Hartley, 1993), a compensatory positive charge was introduced by replacing $\mathrm{Cys}_{42}$ to a lysine. The obtained crystal structure at $1.98 \AA$ resolution revealed that the aspartic acid and lysine side chains are in very close proximity $(2.7 \AA)$ probably forming an additional shielded salt bridge, 
which makes the complex comprised of this mutant protein pair extremely stable.

Barnase seems to have a very stable fold. It can be expressed in a soluble manner to high amounts and it supports other proteins solubility when used as N-terminal fusion tag as in case of full length streptavidin (preliminary data, not shown). By contrast, Barstar has severe problems in solubility when expressed alone. Although its solubility is supported by ZZ-fusion tags, $\mathrm{H}_{14}$-brSUMO or $\mathrm{H}_{14}$-brSUMO-MBP tags do not prevent insolubility of the protein. Barstar contains a parallel $\beta$-sheet located on the opposite site of the Barnase:Barstar interface, which seems to be imperfectly packed. Hydrophobic residues are solvent exposed which makes the entire protein more prone to aggregation. By mutating the $\beta$-sheet region, solubility could be slightly increased, however it still did not result in an overall stable fold. Thus it is not suitable as N-terminal fusion tag, which was also experimentally shown by expression of a Barstar-brSUMO-GFP construct resulting in insoluble protein (data not shown).

Interestingly, although much is known about the binding and thus inhibition of Barnase by Barstar, the question what happens to the complex in the cell in terms of degradation remains unanswered. We speculate that the imperfect folding of the Barstar molecule, which might even be enhanced upon barnase binding, is beneficial for a short protein half-life and thus a quick degradation of the Barnase:Barstar complex, removing a highly toxic protein from the cellular interior. How this degradation occurs, is however unclear. Recently, an ubiquitin - like system was described for Mycobacterium tuberculosis (reviewed in Burns and Darwin, 2010). A prokaryotic ubiquitin-like protein (Pup) can be attached to lysine residues of the target protein with the help of two additional proteins, Dop and PafA, thus targeting the protein to the protease complexes, ClpP respectively. The B.amyloliquefaciens Barstar proteins has two consecutive N-terminal lysine residues, that are also fully exposed when looking at the structure, thus being putative degradation targeting sites.

The Barstar solubility problem was solved by switching from the B.amyloliquefaciens sequence to the sequence identified from the thermophilic Bacillus species G.thermoglucosidasius, which is known to ideally grow at temperatures ranging from $40^{\circ} \mathrm{C}$ to $70^{\circ} \mathrm{C}$ (Nazina et al., 2001). Proteins from thermophilic organisms tend to have a more stable fold and thus could result in stable and soluble barstar protein when expressed and purified alone. Comparing complexes containing different barnase and barstar proteins by Thermofluor revealed that the pair containing not only Barstar but also the corresponding Barnase protein from G.thermoglucosidasius has the highest melting temperature and thus is the most stable complex tested. The elevated melting temperature of the complex can again be explained by the increased stability of the individual proteins. When comparing the Barstar sequences of the two organism, a significant N-terminal extension can be observed 
that potentially folds back onto the structure, thereby shielding the hydrophobic core of the protein that seems to be solvent exposed in B.amyloliquefaciens Barstar. However, full understanding of the fold of G.thermoglucosidasius Barstar required high resolution structures.

\subsubsection{Potential application}

The aim of the project was, to identify a protein pair that can be used for affinity chromatography. The Barnase:Barstar system fulfills all our requirements regarding size, monomeric state and high affinity binding. In addition, the proteins taken from the hyperthermophilic Geobacillus thermoglucosidasius can be expressed in a soluble manner and be produced in high amounts, the strong binding between the affinity pair prevents leakage of the target protein from the resin and background binding towards the affinity proteins is very low.

Frey and Görlich (2014b) published a strategy for stochiometric protein complex purification based on two resins (here $\mathrm{Ni}^{2+}$ and ZZ-affibody resin) and two orthogonal proteases (see figure 4.1). This system can be further expanded also for trimeric complexes by using the Barnase:Barstar system together with an additional orthogonal protease, e.g. based on the Atg8 system (unpublished data). We also intend to use the Barnase:Barstar system as affinity pair during phage display, replacing the streptavidin:biotin system.

Besides affinity chromatography, the Barnase:Barstar pair can also be used to crosslink other molecules without the need of chemical modifications. First approaches in this direction were performed by Deyev et al. (2003), producing a multimeric miniantibody by linking scFv fragments by heterodimeric B.amyloliquefaciens Barnase:Barstar complexes. In principle the concept can be as well used to tether proteins to cellular structures also in vivo, similar to the rapamycin inducible system based in FKBP12 and FRB (Banaszynski et al., 2005).

\subsection{Perspectives}

In this study, we developed and characterized a new affinity resin based on a MADA activated stationary phase and the Barnase:Barstar protein pair as affinity tag system. It remains to be evaluated which of the two proteins is better suited as N-terminal tag or as immobilized stationary phase, respectively. Requirements for a good tag protein would be that the tag itself helps the fusion protein with regards to expression and solubility. Preliminary data suggest that Barnase as N-terminal fusion tag is able to solubilize full length streptavidin, a protein that can be solubly expressed when fused to larger tags but remains insoluble when expressed with small tags such as $\mathrm{H}_{14}$-brSUMO only. Similar 
experiments need to be performed with Barstar as fusion protein. Good characteristics for the stationary phase protein are a stable fold, that might even be able to tolerate denaturation and subsequent renaturation. This would enable to recover the resin after use. Currently, we examine the ability of a Barstar Sepharose and a Barnase Sepharose to maintain their binding ability towards their corresponding partner even after thorough washing with guanidine hydrochloride or SDS. The MADA Sepharose itself needs to be further analyzed for ideal quenching components and long-term storage conditions.

In conjunction with the MADA resin, we would like to perform binding assays and pulldown experiments, exploiting the extremely low background binding, the strong affinity of the tag system and the ability to purify also large complexes in a native manner. We aim to genetically modify bacterial ribosomes with the Barnase or Barstar tag, by fusing them to the most exterior ribosomal proteins such as L1 protein. The tag would be extremely small with either $13 \mathrm{kDa}$ or $11 \mathrm{kDa}$, decreasing the risk that the tag interferes with the function of the ribosome. Pulling out the tagged ribosomes with MADA activated magnetic beads containing the immobilized affinity partner and subsequent elution using proteases would enable to purify ribosomes under native and rather mild conditions in a one-step manner, circumventing tedious purification protocols using density centrifugation, respectively. If the procedure is successful, similar strategies can be applied for the purification of entire organelles such as mitochondria.

In addition, we identified another potential affinity tag system based on the plasmidencoded antibacterial protein Colicin produced by some bacteria, such as E.coli. On the same plasmid, immunity proteins are encoded in order to protect the bacteria against the cytotoxic activity of the colicin. The affinity between the proteins is considered to be one of the highest measured affinities between two proteins with a equilibrium dissociation constant of $9.3 \times 10^{-17} \mathrm{M}$ (reviewed in Kolade et al., 2002). So far preliminary experiments with the non-toxic C-terminal domain of colicin E9 and its full length immunity protein 9 were performed, showing that both of them can be expressed in E.coli and further purified. Binding assays revealed that the recombinant proteins bind to each other.

Finally we aim to share our findings with the scientific community, thereby giving a guide how to easily produce a reasonable and tailor-made affinity resin for an extremely broad range of individual applications. Additionally, we aim to establish the Barnase:Barstar system as beneficial alternative to the - partially - disadvantageous streptavidin:biotin system. 


\section{Materials and Methods}

\subsection{Materials}

\subsubsection{Chemicals and Reagents}

If not stated otherwise, all chemical were purchased to the highest available purity from Calbiochem (San Diego, California, USA), Gibco Life Technologies (Paisley, United Kingdom), Merck (Darmstadt, Germany), MoBiTech (Göttingen, Germany), Pharmacia (Uppsala, Sweden), Pierce (part of ThermoScientific, Rockford, Illinois, USA), Promega (Madison, Wisconsin, USA), Quiagen (Hilden, Germany), Roche Diagnostics (Mannheim, Germany), Carl Roth (Karlsruhe, Germany) or Sigma-Aldrich (St. Louis, Missouri, USA).

\subsubsection{Instruments}

$\begin{array}{ll}\text { ÄKTA Purifyer } & \text { Pharmacia, Upsala, Schweden } \\ \text { Biophotometer-plus } & \text { Eppendorf AG, Hamburg, Germany } \\ \text { BioTek Synergy H4 Plate Reader } & \text { BioTek, Friedrichshall, Germany } \\ \text { Climo - Shaker ISF1-X } & \text { Kuhner AG, Basel, Switzerland } \\ \text { FLA-7000 Scanner } & \text { Fujifilm, Tokio, Japan } \\ \text { GenePulser }{ }^{T} M & \text { BioRad, Hercules, California, USA } \\ \text { Microlab Star Pipetting Robot } & \text { Hamilton Robotics, Bonaduz, Switzerland } \\ \text { iMac 3.1 GHz Intel Core i5 } & \text { Apple, Cupertino, California, USA } \\ \text { NanoDrop 2000c } & \text { PeqLab, Erlangen, Germany } \\ \text { Nikon D300 } & \text { Nikon, Tokio, Japan } \\ \text { Odyssey } & \text { Licor, Lincoln, Nebraska, USA } \\ \text { Perfection V700 Photo Scanner } & \text { Epson, Tokio, Japan } \\ \text { Pipetman pipettes } & \text { Gilson, Middleton, Wisconsin, USA } \\ \text { SensoQuest Lab Cycler } & \text { SensoQuest, Göttingen, Germany } \\ \text { Sieving machine AS300 } & \text { Retsch, Germany } \\ \text { Sonifier 450 } & \text { Branson, United Kingdom } \\ \text { Thermomixer comfort } & \text { Eppendorf AG, Hamburg, Germany } \\ \text { Windsichter Taifun } 200 & \text { ISS Schüttguttechnik GmbH, Wilnsdorf, Germany } \\ \end{array}$


Centrifuges

Cooled tabletop centrifuge

Tabletop centrifuge

Multifuge 3L-R

RC6 Plus centrifuge

WX Ultra centrifuge

Discovery M120
Rotors

$-$

$-$

$-$

F9, F10, F14

T647.5, T125.0

S45A, S55S

\section{Company}

Eppendorf AG, Hamburg, Germany

Eppendorf AG, Hamburg, Germany

Thermo Scientific, Massachusetts, USA

Sorvall/Thermo Scientific, Massachusetts, USA

Sorvall/Thermo Scientific, Massachusetts, USA

Sorvall/Thermo Scientific, Massachusetts, USA

\subsubsection{Software}

Bookends 11.3.7

ChemSketch 14.0

GeneDesigner 2

Illustrator CS3 $/ 5$

Lasergene 8

$\mathrm{IAT}_{\mathrm{E}} \mathrm{X}$

Mac OS X Vers.10.6.7

Microsoft Office

Oligo 7

Photoshop CS3 $/ 5$

Prism 5

$\mathrm{MacPyMol}$

Scaffold 3
Sonny Software, Chevy Chase, Maryland, USA

Advanced Chemistry Development, Inc., Toronto, Canada

DNA2.0, Menlo Park, California, USA

Adobe Systems, San Jose, California, USA

DNA-Star, Madison, Wisconsin, USA

http://www.latex-pro ject.org/

Apple, Cupertino, California, USA

Microsoft Cooperation, Champaign, Illinois, USA

Molecular Biology Insights, Cascade, Colorado, USA

Adobe Systems, San Jose, California, USA

GraphPad Software, La Jolla, California, USA

Schrödinger, Portland, Oregon, USA

Proteome Software, Portland, Oregon, USA

\subsubsection{Bioinformatic Resources}

BLAST (Basic Local Alignment Search Tool)

NCBI protein database

PDB (Protein Data Bank)

PFAM protein families database

Smart (Simple Modular Architecture Research Tool)

UniProtKB/Swiss-Prot

WolframAlpha http://blast.ncbi.nlm.nih.gov

http://www.ncbi.nlm.nih.gov/protein

http://www.rcsb.org/pdb

http://pfam.xfam.org

http://smart.embl-heidelberg.de

http://web.expasy.org

https://www.wolframalpha.com 


\subsubsection{Escherichia coli medium}

\section{LB medium 2YT medium}

10 g Tryptone

$5 \mathrm{~g}$ Yeast extract

$10 \mathrm{~g} \mathrm{NaCl}$
SOB

100 g Tryptone

25 g Yeast extract

$2.5 \mathrm{~g} \mathrm{NaCl}$

$0.93 \mathrm{~g} \mathrm{KCl}$$$
\mathrm{g} \mathrm{KCl}
$$

\section{TB}

12g Tryptone

$24 \mathrm{~g}$ Yeast extract

$5 \mathrm{~g} \mathrm{NaCl}$ 4ml Glycerol

$2.13 \mathrm{~g} \mathrm{KH}_{2} \mathrm{PO}_{4}$

$12.54 \mathrm{~g} \mathrm{~K}_{2} \mathrm{HPO}_{4}$

$\mathrm{ddH}_{2} \mathrm{O}$ to $1 \mathrm{~L} \quad \mathrm{ddH}_{2} \mathrm{O}$ to $1 \mathrm{~L} \quad \mathrm{ddH}_{2} \mathrm{O}$ to $5 \mathrm{~L} \quad \mathrm{ddH}_{2} \mathrm{O}$ to $1 \mathrm{~L}$

For the pouring of agar plates, $15 \mathrm{~g}$ agar were added to $1 \mathrm{~L}$ of medium. For selection and culturing of bacteria after transformation, 2TY plates were supplemented with the appropriate antibiotics in the following concentrations: Ampicillin $(100 \mu \mathrm{g} / \mathrm{ml})$, Kanamycin $(25 \mu \mathrm{g} / \mathrm{ml})$,Spectinomycin $(50 \mu \mathrm{g} / \mathrm{ml})$ or Chloramphenicol $(50 \mu \mathrm{g} / \mathrm{ml})$. The expression cultures were grown in either $2 \mathrm{YT}$ or $\mathrm{TB}$ medium supplemented with the appropriate antibiotics.

\subsubsection{Escherichia coli strains}

Table 4.1: E.coli strains used in this study

\begin{tabular}{|c|c|c|}
\hline Strain & Genotype & Company \\
\hline BL21 DE3 Star & $\begin{array}{l}\text { F-ompT gal dem lon hsdSB(rB-mB-) } \lambda(\text { DE3 [lacI } \\
\text { lacUV5-T7 gene } 1 \text { ind1 sam7 nin5]) }\end{array}$ & $\begin{array}{l}\text { Invitrogen } \\
\text { (Karlsruhe) }\end{array}$ \\
\hline BLR & $\begin{array}{l}\text { F-ompT hsdSB (rB-mB-) } \\
\text { recA)306::Tn10 }\left(\text { Tet }^{R}\right)\end{array}$ & $\begin{array}{l}\text { Invitrogen } \\
\text { (Karlsruhe) }\end{array}$ \\
\hline $\mathrm{NEB} 5 \alpha \mathrm{F}^{\prime}$ & $\begin{array}{l}\mathrm{F}^{\prime} \text { proA+B+ lacIq } \Delta(\text { lacZ)M15 zzf::Tn10 } \\
\left(\text { Tet }^{R}\right) \text { / fhuA2 } \Delta(\operatorname{argF-lacZ)U169~phoA~glnV44~} \\
\phi 80 \Delta(\text { lacZ)M15 gyrA96 recA1 relA1 endA1 thi-1 } \\
\text { hsdR17 }\end{array}$ & $\begin{array}{l}\text { NEB } \\
\text { (Beverly, } \\
\text { USA) }\end{array}$ \\
\hline NEB10 $\beta \mathrm{F}^{\prime}$ & $\begin{array}{l}\text { F' } \Delta \text { (ara-leu) } 7697 \text { araD139 fhuA } \Delta \text { lacX74 galK16 } \\
\text { galE15 e14- } \phi 80 \text { dlacZ } \Delta \text { M15 recA1 relA1 endA1 nupG } \\
\text { rpsL }\left(\operatorname{Str}^{R}\right) \text { rph spoT1 } \Delta(\text { mrr-hsdRMS-mcrBC })\end{array}$ & $\begin{array}{l}\text { NEB } \\
\text { (Beverly, } \\
\text { USA) }\end{array}$ \\
\hline NEB Express & $\begin{array}{l}\text { fhuA2 [lon] ompT gal sulA11 R(mcr-73::miniTn10- } \\
\left.\text { TetS } 2 \text { [dcm] R(zgb-210::Tn10-Tet }{ }^{S}\right) \text { endA1 } \Delta(\text { mcrC- } \\
\text { mrr)114::IS10 }\end{array}$ & $\begin{array}{l}\text { NEB } \\
\text { (Beverly, } \\
\text { USA) }\end{array}$ \\
\hline TOP10F' & $\begin{array}{l}\text { F' }^{\prime}\left[\mathrm{lac}^{Q} \text {, Tn10 } \text { Tet }^{R}\right] \text { mcrA } \Delta(\text { mrr-hsdRMS-mcrBC), } \\
\text { 80lacZ } \Delta \text { M15 } \Delta \text { lacX74, deoR, recA1, araD139 (ara- } \\
\text { leu) } 7679 \text {, galU, galK, rpsL }\left(\mathrm{Str}^{R}\right) \text {, endA1, nupG }\end{array}$ & $\begin{array}{l}\text { Invitrogen } \\
\text { (Karlsruhe) }\end{array}$ \\
\hline
\end{tabular}




\subsubsection{Standard Buffers}

10x PfuS polymerase buffer:

$20 \mathrm{mM}$ Tris- $\mathrm{HCl}$ pH9.0, 250mM KCl, $15 \mathrm{mM} \mathrm{MgSO}_{4}, 100 \mathrm{mM}\left(\mathrm{NH}_{4}\right)_{2} \mathrm{SO}_{4}, 1 \%$ Tween20, $1 \mathrm{mg} / \mathrm{ml} \mathrm{BSA}$

10x Ligation buffer:

$500 \mathrm{mM}$ Tris pH7.5, 100mM $\mathrm{MgCl}_{2}, 100 \mathrm{mM}$ DTT, $10 \mathrm{mM}$ ATP, $250 \mu \mathrm{g} / \mathrm{mL}$ BSA

\section{Orange G DNA loading buffer:}

10mM Tris-HCl pH8.0, 10mM EDTA pH8.0, 50\% Glycerol (w/v), 0.25\% OrangeG (w/v)

\section{DNA ladder:}

$50 \mathrm{ng} / \mu \mathrm{l}$ 1kb-Ladder or 50ng/ $\mu \mathrm{l} 100 \mathrm{~kb}-$ Ladder in Orange sample buffer

50x TAE:

242g Tris Base, $57.1 \mathrm{~mL}$ acetic acid, $100 \mathrm{~mL}$ 0.5M EDTA pH8.0, add to $1 \mathrm{~L}$ with $\mathrm{ddH}_{2} 0$

\section{Tris/HCl pH 7.5:}

Tris buffers were prepared according to Henderson-Hasselbach equation $\left(\mathrm{pH}=\mathrm{pK}_{\mathrm{s}}+\right.$ $\log (\mathrm{S} / \mathrm{B}))$. Prior to usage, the $\mathrm{pH}$ was measured and, if necessary, manually adjusted.

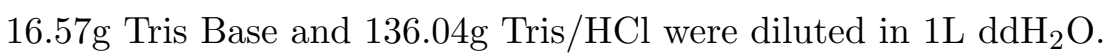

\subsection{EDTA pH 8.0:}

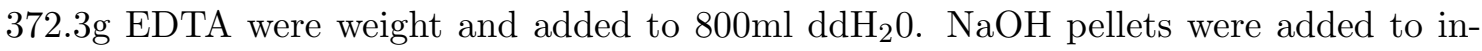
crease the $\mathrm{pH}$ and thus allow EDTA to solve. Finally, the volume was adjusted to $1 \mathrm{~L}$ with $\mathrm{ddH}_{2} \mathrm{O}$.

\section{SDS Sample Buffer:}

$3 \%$ SDS, 125mM Tris/HCl pH6.8, 50mM DTT, 1M sucrose, Bromphenolblue

\section{0x SDS-PAGE running buffer:}

300g Glycine, 60g Tris Base, 24g SDS, add to 2L with $\mathrm{ddH}_{2} 0$.

\section{Coomassie stock solution:}

$2 \%(\mathrm{w} / \mathrm{v})$ Coomassie brilliant blue G250 in 50\% Ethanol 


\section{Colloidal Coomassie stock solution:}

0.08\% (w/v) Coomassie brilliant blue G250, 1.6\% (v/v) ortho-Phosphoric acid, $8 \%$ (w/v) Ammonium sulfate, $20 \%$ (v/v) Methanol

\section{0x Blotting Buffer:}

100ml 10x SDS-PAGE running buffer, $200 \mathrm{ml} \mathrm{MeOH,} \mathrm{0.03 \%} \mathrm{SDS,} \mathrm{add} \mathrm{to} 1 \mathrm{~L}$ with $\mathrm{ddH}_{2} 0$

\section{0x TBS Buffer:}

$50 \mathrm{~g}$ Tris/HCl, 14.5g Tris Base, 175.2g NaCl, add 20 1L with $\mathrm{ddH}_{2} 0$

10x PBS (pH 7.4):

$137 \mathrm{mM} \mathrm{NaCl}, 2.7 \mathrm{mM} \mathrm{KCl}, 8.1 \mathrm{mM} \mathrm{Na} 2 \mathrm{HPO}_{4}, 1.76 \mathrm{mM} \mathrm{KH}_{2} \mathrm{PO}_{4}$

\section{Fixation Buffer:}

$50 \% \mathrm{MeOH}, 40 \% \mathrm{ddH}_{2} 0,10 \%$ Acetic Acid

\section{0x E-mix:}

20mM HEPES pH7.5, 250mM Sucrose, 200mM Creatine phosphate, 10mM ATP, 10mM GTP, $1 \mathrm{mg} / \mathrm{ml}$ Creatine kinase

\section{0x CPAG:}

0.331g ATP, $0.0654 \mathrm{~g}$ GTP, $2.616 \mathrm{~g}$ creatine phosphate, fill up to $9 \mathrm{ml}$ with $\mathrm{ddH}_{2} 0$, adjust $\mathrm{pH}$ to $\mathrm{pH} 7.8$ with $\mathrm{KOH}$, then fill up to $10 \mathrm{ml}$ with $\mathrm{ddH}_{2} 0$.

\subsubsection{Oligonucleotides}

The oligonucleotides used in this study were designed using the Oligo 6.8 (Molecular Biology Insights Inc., Cascade, Colorado, USA) software and synthesized by Sigma-Aldrich (St. Louis, Missouri, USA) or IBA GmbH (Göttingen, Germany). Standard oligos were purified by desalting. HPLC-purified and 5'-phosphorylated oligos were used for mutagenesis PCR and cloning with annealed oligos.

\subsubsection{Gene Synthesis}

Several genes encoding proteins used in this study were designed for optimized expression in E.coli using the GeneDesigner 2 (DNA2.0) software. In addition, these constructs were rendered such that they lack internal ribosome binding sites or commonly used restric- 
tion enzyme recognition sites. The constructs were de novo synthesized by GeneScript (Piscataway, New Jersey, USA).

\subsubsection{Antibodies}

The following antibodies were used in this study.

Table 4.2: Antibodies used in this study

Antibodies against translation factors were purified from rabbit sera. Antigens for rabbit immunization and sera preparation was performed by Dr. Cathrin Enke. Antibodies against translation factors were raised against Triticum aestivum proteins (if not stated otherwise). Commercial antibodies were used as stated in the manufacturers instructions.

\begin{tabular}{|c|c|c|c|c|}
\hline Antibody & Туре & $\begin{array}{c}\text { Concentration } \\
\text { in WB }\end{array}$ & $\begin{array}{c}\text { used } \\
\text { in IPs }\end{array}$ & Reference \\
\hline$\alpha$-eIF3a & primary & $1 \mu \mathrm{g} / \mathrm{ml}$ & & This study \\
\hline$\alpha$-eIF3b & primary & $1 \mu \mathrm{g} / \mathrm{ml}$ & yes & This study \\
\hline$\alpha$-eIF3c & primary & $1 \mu \mathrm{g} / \mathrm{ml}$ & yes & This study \\
\hline$\alpha$-eIF3d & primary & $1 \mu \mathrm{g} / \mathrm{ml}$ & yes & This study \\
\hline$\alpha$-eIF3e & primary & $1 \mu \mathrm{g} / \mathrm{ml}$ & & This study \\
\hline$\alpha$-eIF3f & primary & $1 \mu \mathrm{g} / \mathrm{ml}$ & & This study \\
\hline$\alpha$-eIF3g & primary & $1 \mu \mathrm{g} / \mathrm{ml}$ & & This study \\
\hline$\alpha$-eIF3h & primary & $1 \mu \mathrm{g} / \mathrm{ml}$ & & This study \\
\hline$\alpha$-eIF3i & primary & $1 \mu \mathrm{g} / \mathrm{ml}$ & & This study \\
\hline$\alpha$-eIF $3 \mathrm{j}$ & primary & $1 \mu \mathrm{g} / \mathrm{ml}$ & & This study \\
\hline$\alpha$-eIF3k & primary & $1 \mu \mathrm{g} / \mathrm{ml}$ & & This study \\
\hline$\alpha$-eIF3L & primary & $1 \mu \mathrm{g} / \mathrm{ml}$ & & This study \\
\hline$\alpha$-eIF3m & primary & $1 \mu \mathrm{g} / \mathrm{ml}$ & yes & This study \\
\hline$\alpha$-eIF1 & primary & - & yes & Enke (2010) \\
\hline$\alpha$-eIF $2 \alpha$ & primary & $1 \mu \mathrm{g} / \mathrm{ml}$ & yes & Enke (2010) \\
\hline$\alpha$-eIF $2 \beta$ & primary & $1 \mu \mathrm{g} / \mathrm{ml}$ & & Enke (2010) \\
\hline$\alpha$-eIF2B $\beta$ & primary & - & & Enke (2010) \\
\hline$\alpha$-eIF5 & primary & $1 \mu \mathrm{g} / \mathrm{ml}$ & & Enke (2010) \\
\hline$\alpha$-eEF $1 \mathrm{~B} \beta$ & primary & $1 \mu \mathrm{g} / \mathrm{ml}$ & & Enke (2010) \\
\hline$\alpha$-hs eIF5A N-terminal & primary & $1 \mu \mathrm{g} / \mathrm{ml}$ & & $\begin{array}{l}\text { Lipowsky } \\
\text { et al. }(2000)\end{array}$ \\
\hline$\alpha$-hs eIF5A internal & primary & $1 \mu \mathrm{g} / \mathrm{ml}$ & & $\begin{array}{l}\text { Lipowsky } \\
\text { et al. }(2000)\end{array}$ \\
\hline$\alpha$-GFP & primary & $1 \mu \mathrm{g} / \mathrm{ml}$ & yes & $\begin{array}{c}\text { D. Görlich } \\
\text { (unpublished) }\end{array}$ \\
\hline$\alpha$-M13 pIII monoclonal & primary & $1: 1000$ & & NEB \\
\hline$\alpha$-Flag M2 monoclonal & primary & $1: 1000$ & & SIGMA \\
\hline$\alpha-\mathrm{HA}(\mathrm{Y}-11): \mathrm{sc}-805$ & primary & 1:1000 & & $\begin{array}{c}\text { Santa Cruz } \\
\text { Biotechnology }\end{array}$ \\
\hline
\end{tabular}




\begin{tabular}{|l||l|l|l|l|}
\hline IRDye 800CW Goat $\alpha$-Rabbit & secondary & $1: 50.000$ & & Licor, USA \\
\hline IRDye 700CW Goat $\alpha$-Mouse & secondary & $1: 50.000$ & & Licor, USA \\
\hline
\end{tabular}

\subsubsection{Plasmids}

All plasmids used in this study were cloned using the standard techniques in molecular biology described in 4.2.1. Plasmids were used either for in vitro translation (see table 4.3) or for protein expression in E.coli (see table 4.4).

\section{Table 4.3: In vitro translation plasmids used in this study}

All constructs described are based on the same backbone, containing an N-terminal T7 promotor and a 5'UTR based on Kozak (1994). At the end of the open reading frame (ORF), a TMV 3' translation enhancer was introduced followed by a poly-A tail and the T7 terminator. All constructs harbor a kanamycin resistance. The backbone was constructed by Dr. Cathrin Enke. For details see Enke (2010).

\begin{tabular}{|c|c|c|}
\hline Plasmid number & Construct & Reference \\
\hline T38 & $\mathrm{H}_{21}-\mathrm{TEV}$-Firefly Luciferase & Dissertation (Enke, 2010) \\
\hline JS250 & $\mathrm{H}_{10}-12 \mathrm{xPro}-\mathrm{MS}$ stretch-Nup98FG $(\mathrm{P} \rightarrow \mathrm{S}$, short $)$ & This study \\
\hline JS251 & $\mathrm{H}_{10}-12 \mathrm{xSer}-\mathrm{MS}$ stretch-Nup98FG $\mathrm{P}_{(\mathrm{P} \rightarrow \mathrm{S}}$, long $)$ & This study \\
\hline JS331 & $3 \mathrm{xFLAG}-12 \mathrm{xPro}-\mathrm{MS}$ stretch-Nup98FG $(\mathrm{P} \rightarrow \mathrm{S}$, short $)$ & This study \\
\hline JS332 & 3x-FLAG-12xSer-MS stretch-Nup98FG $(\mathrm{P} \rightarrow \mathrm{S}$, long $)$ & This study \\
\hline JS359 & 3x-FLAG-12xSer-Firefly Luciferase & This study \\
\hline JS425 & 3x-FLAG-S.c.-Ldb17 & This study \\
\hline JS426 & 3x-FLAG-S.c.-Eap1 & This study \\
\hline
\end{tabular}


Table 4.4: E.coli expression vectors used in this study

The table lists the most important parameters when expressing and purifying the mentioned constructs. $\mathrm{T}=\mathrm{Tris} / \mathrm{HCl}, \mathrm{pH} 7.5 ; \mathrm{N}=\mathrm{NaCl} ; \mathrm{l}=\mathrm{Imidazole}$;

$\mathrm{D}=\mathrm{DTT} ; \mathrm{M}=\mathrm{MgCl}_{2}, \mathrm{H}=\mathrm{Hepes} / \mathrm{KOH}, \mathrm{pH}$ 7.4. Protease elution was performed on-column with either SENP1 protease or NEDP1 protease.

\begin{tabular}{|c|c|c|c|c|c|c|c|c|c|c|c|}
\hline \multirow[b]{2}{*}{ Plasmid } & \multirow[b]{2}{*}{ Construct } & \multirow[b]{2}{*}{ Marker } & \multirow[b]{2}{*}{ Ori } & \multicolumn{4}{|c|}{ Expression conditions } & \multicolumn{3}{|c|}{ Purification Conditions } & \multirow[b]{2}{*}{ Reference } \\
\hline & & & & Temp. & Duration & Strain & $\begin{array}{c}\text { IPTG } \\
(\mu \mathbf{M})\end{array}$ & buffer & Elution & $\begin{array}{l}\text { Gel- fil- } \\
\text { tration }\end{array}$ & \\
\hline pJS040 & $\mathrm{H}_{14}$-eIF3a & Kan & ColE1 & $18^{\circ} \mathrm{C}$ & $\mathrm{o} / \mathrm{n}$ & Top10F' & 100 & - & - & - & This study \\
\hline pJS041 & $\mathrm{H}_{14}$-eIF3b & Kan & ColE1 & $18^{\circ} \mathrm{C}$ & $\mathrm{o} / \mathrm{n}$ & Top10F' & 100 & $50 \mathrm{~T}, 500 \mathrm{~N}, 2 \mathrm{I}, 1 \mathrm{D}$ & $500 \mathrm{I}$ & - & This study \\
\hline pJS042 & $\mathrm{H}_{14}$-eIF3c & Kan & ColE1 & $18^{\circ} \mathrm{C}$ & $\mathrm{o} / \mathrm{n}$ & Top10F' & 100 & $50 \mathrm{~T}, 500 \mathrm{~N}, 2 \mathrm{I}, 1 \mathrm{D}$ & $500 \mathrm{I}$ & - & This study \\
\hline pJS043 & $\mathrm{H}_{14}$-eIF3d & Kan & ColE1 & $18^{\circ} \mathrm{C}$ & $\mathrm{o} / \mathrm{n}$ & Top10F' & 100 & $50 \mathrm{~T}, 500 \mathrm{~N}, 2 \mathrm{I}, 1 \mathrm{D}$ & $500 \mathrm{I}$ & - & This study \\
\hline pJS044 & $\mathrm{H}_{14}$-eIF3e & Kan & ColE1 & $18^{\circ} \mathrm{C}$ & $\mathrm{o} / \mathrm{n}$ & Top10F' & 100 & - & - & - & This study \\
\hline pJS045 & $\mathrm{H}_{14}$-eIF3f & Kan & ColE1 & $18^{\circ} \mathrm{C}$ & $\mathrm{o} / \mathrm{n}$ & Top10F' & 100 & - & - & - & This study \\
\hline pJS046 & $\mathrm{H}_{14}$-eIF3g & Kan & ColE1 & $18^{\circ} \mathrm{C}$ & $\mathrm{o} / \mathrm{n}$ & Top10F' & 100 & - & - & - & This study \\
\hline pJS047 & $\mathrm{H}_{14}$-eIF3h & Kan & ColE1 & $18^{\circ} \mathrm{C}$ & $\mathrm{o} / \mathrm{n}$ & Top10F' & 100 & $50 \mathrm{~T}, 500 \mathrm{~N}, 2 \mathrm{I}, 1 \mathrm{D}$ & $500 \mathrm{I}$ & - & This study \\
\hline pJS048 & $\mathrm{H}_{14}$-eIF3i & Kan & ColE1 & $18^{\circ} \mathrm{C}$ & $\mathrm{o} / \mathrm{n}$ & Top10F' & 100 & - & - & - & This study \\
\hline pJS049 & $\mathrm{H}_{14}$-eIF3j & Kan & ColE1 & $18^{\circ} \mathrm{C}$ & $\mathrm{o} / \mathrm{n}$ & Top10F' & 100 & $50 \mathrm{~T}, 500 \mathrm{~N}, 2 \mathrm{I}, 1 \mathrm{D}$ & $500 \mathrm{I}$ & - & This study \\
\hline pJS0410 & $\mathrm{H}_{14}$-eIF3k & Kan & ColE1 & $18^{\circ} \mathrm{C}$ & $\mathrm{o} / \mathrm{n}$ & Top10F' & 100 & $50 \mathrm{~T}, 500 \mathrm{~N}, 2 \mathrm{I}, 1 \mathrm{D}$ & $500 \mathrm{I}$ & - & This study \\
\hline pJS0411 & $\mathrm{H}_{14}$-eIF3L & Kan & ColE1 & $18^{\circ} \mathrm{C}$ & $\mathrm{o} / \mathrm{n}$ & Top10F' & 100 & - & - & - & This study \\
\hline pJS0412 & $\mathrm{H}_{14}$-eIF3m & Kan & ColE1 & $18^{\circ} \mathrm{C}$ & $\mathrm{o} / \mathrm{n}$ & Top10F' & 100 & - & - & - & This study \\
\hline $\mathrm{T} 238$ & $\mathrm{H}_{14}$-TEV-eIF3a & Kan & ColE1 & $18^{\circ} \mathrm{C}$ & $\mathrm{o} / \mathrm{n}$ & Top10F' & 100 & - & - & - & Enke (2010) \\
\hline T209 & $\mathrm{H}_{14}$-TEV-eIF3b & Kan & ColE1 & $18^{\circ} \mathrm{C}$ & $\mathrm{o} / \mathrm{n}$ & Top10F' & 100 & $50 \mathrm{~T}, 500 \mathrm{~N}, 2 \mathrm{I}, 1 \mathrm{D}$ & $500 \mathrm{I}$ & - & Enke (2010) \\
\hline $\mathrm{T} 208$ & $\mathrm{H}_{14}$-TEV-eIF3c & Kan & ColE1 & $18^{\circ} \mathrm{C}$ & $\mathrm{o} / \mathrm{n}$ & Top10F' & 100 & $50 \mathrm{~T}, 500 \mathrm{~N}, 2 \mathrm{I}, 1 \mathrm{D}$ & $500 \mathrm{I}$ & - & Enke (2010) \\
\hline $\mathrm{T} 207$ & $\mathrm{H}_{14}$-TEV-eIF3d & Kan & ColE1 & $18^{\circ} \mathrm{C}$ & $\mathrm{o} / \mathrm{n}$ & Top10F' & 100 & $50 \mathrm{~T}, 500 \mathrm{~N}, 2 \mathrm{I}, 1 \mathrm{D}$ & $500 \mathrm{I}$ & - & Enke (2010) \\
\hline T206 & $\mathrm{H}_{14}$-TEV-eIF3e & Kan & ColE1 & $18^{\circ} \mathrm{C}$ & $\mathrm{o} / \mathrm{n}$ & Top10F' & 100 & - & - & - & Enke (2010) \\
\hline $\mathrm{T} 242$ & $\mathrm{H}_{14}$-TEV-eIF3f & Kan & ColE1 & $18^{\circ} \mathrm{C}$ & $\mathrm{o} / \mathrm{n}$ & Top10F' & 100 & - & - & - & Enke (2010) \\
\hline $\mathrm{T} 205$ & $\mathrm{H}_{14}$-TEV-eIF3g & Kan & ColE1 & $18^{\circ} \mathrm{C}$ & $\mathrm{o} / \mathrm{n}$ & Top10F' & 100 & - & - & - & Enke (2010) \\
\hline $\mathrm{T} 204$ & $\mathrm{H}_{14}$-TEV-eIF3h & Kan & ColE1 & $18^{\circ} \mathrm{C}$ & $\mathrm{o} / \mathrm{n}$ & Top10F' & 100 & $50 \mathrm{~T}, 500 \mathrm{~N}, 2 \mathrm{I}, 1 \mathrm{D}$ & $500 \mathrm{I}$ & - & Enke (2010) \\
\hline T202 & $\mathrm{H}_{14}$-TEV-eIF3i & Kan & ColE1 & $18^{\circ} \mathrm{C}$ & $\mathrm{o} / \mathrm{n}$ & Top10F' & 100 & - & - & - & Enke (2010) \\
\hline $\mathrm{T} 203$ & $\mathrm{H}_{14}$-TEV-eIF3j & Kan & ColE1 & $18^{\circ} \mathrm{C}$ & $\mathrm{o} / \mathrm{n}$ & Top10F' & 100 & $50 \mathrm{~T}, 500 \mathrm{~N}, 2 \mathrm{I}, 1 \mathrm{D}$ & $500 \mathrm{I}$ & - & Enke (2010) \\
\hline T181 & $\mathrm{H}_{14}$-TEV-eIF3k & Kan & ColE1 & $18^{\circ} \mathrm{C}$ & $\mathrm{o} / \mathrm{n}$ & Top10F' & 100 & $50 \mathrm{~T}, 500 \mathrm{~N}, 2 \mathrm{I}, 1 \mathrm{D}$ & $500 \mathrm{I}$ & - & Enke (2010) \\
\hline $\mathrm{T} 210$ & $\mathrm{H}_{14}$-TEV-eIF3L & Kan & ColE1 & $18^{\circ} \mathrm{C}$ & $\mathrm{o} / \mathrm{n}$ & Top10F' & 100 & - & - & - & Enke (2010) \\
\hline
\end{tabular}


Table 4.4-Continued from previous page

\begin{tabular}{|c|c|c|c|c|c|c|c|c|c|c|c|}
\hline \multirow[b]{2}{*}{ Plasmid } & \multirow[b]{2}{*}{ Construct } & \multirow[b]{2}{*}{ Marker } & \multirow[b]{2}{*}{ Ori } & \multicolumn{4}{|c|}{ Expression conditions } & \multicolumn{3}{|c|}{ Purification Conditions } & \multirow[b]{2}{*}{ Reference } \\
\hline & & & & Temp. & Duration & Strain & $\begin{array}{c}\text { IPTG } \\
(\mu \mathbf{M})\end{array}$ & buffer & Elution & $\begin{array}{l}\text { Gel- fil- } \\
\text { tration }\end{array}$ & \\
\hline $\mathrm{T} 240$ & $\mathrm{H}_{14}$-TEV-eIF3m & Kan & ColE1 & $18^{\circ} \mathrm{C}$ & $\mathrm{o} / \mathrm{n}$ & Top10F' & 100 & - & - & - & Enke (2010) \\
\hline JS001 & $\begin{array}{c}\mathrm{H}_{14} \text {-brSUMO- } \\
\text { eIF3a }\end{array}$ & Kan & ColE1 & $18^{\circ} \mathrm{C}$ & $\mathrm{o} / \mathrm{n}$ & Top10F' & 100 & - & - & - & This study \\
\hline JS002 & $\begin{array}{c}\mathrm{H}_{14} \text {-brSUMO- } \\
\text { eIF3b }\end{array}$ & Kan & ColE1 & $18^{\circ} \mathrm{C}$ & $\mathrm{o} / \mathrm{n}$ & Top10F' & 100 & $50 \mathrm{~T}, 500 \mathrm{~N}, 2 \mathrm{I}, 1 \mathrm{D}$ & $500 \mathrm{I}$ & - & This study \\
\hline JS003 & $\begin{array}{c}\mathrm{H}_{14} \text {-brSUMO- } \\
\text { eIF3c }\end{array}$ & Kan & ColE1 & $18^{\circ} \mathrm{C}$ & $\mathrm{o} / \mathrm{n}$ & Top10F' & 100 & $50 \mathrm{~T}, 500 \mathrm{~N}, 2 \mathrm{I}, 1 \mathrm{D}$ & $500 \mathrm{I}$ & - & This study \\
\hline JS004 & $\begin{array}{c}\mathrm{H}_{14} \text {-brSUMO- } \\
\text { eIF3d }\end{array}$ & Kan & ColE1 & $18^{\circ} \mathrm{C}$ & $\mathrm{o} / \mathrm{n}$ & Top10F' & 100 & $50 \mathrm{~T}, 500 \mathrm{~N}, 2 \mathrm{I}, 1 \mathrm{D}$ & $500 \mathrm{I}$ & - & This study \\
\hline JS005 & $\begin{array}{c}\mathrm{H}_{14} \text {-brSUMO- } \\
\text { eIF3e }\end{array}$ & Kan & ColE1 & $18^{\circ} \mathrm{C}$ & $\mathrm{o} / \mathrm{n}$ & Top10F' & 100 & - & - & - & This study \\
\hline JS006 & $\begin{array}{c}\mathrm{H}_{14} \text {-brSUMO- } \\
\text { eIF3f }\end{array}$ & Kan & ColE1 & $18^{\circ} \mathrm{C}$ & $\mathrm{o} / \mathrm{n}$ & Top10F' & 100 & - & - & - & This study \\
\hline JS007 & $\begin{array}{c}\mathrm{H}_{14} \text {-brSUMO- } \\
\text { eIF3g }\end{array}$ & Kan & ColE1 & $18^{\circ} \mathrm{C}$ & $\mathrm{o} / \mathrm{n}$ & Top10F' & 100 & $50 \mathrm{~T}, 500 \mathrm{~N}, 2 \mathrm{I}, 1 \mathrm{D}$ & $500 \mathrm{I}$ & - & This study \\
\hline JS008 & $\begin{array}{c}\mathrm{H}_{14} \text {-brSUMO- } \\
\text { eIF3h }\end{array}$ & Kan & ColE1 & $18^{\circ} \mathrm{C}$ & $\mathrm{o} / \mathrm{n}$ & Top10F' & 100 & $50 \mathrm{~T}, 500 \mathrm{~N}, 2 \mathrm{I}, 1 \mathrm{D}$ & $500 \mathrm{I}$ & - & This study \\
\hline JS009 & $\begin{array}{c}\mathrm{H}_{14} \text {-brSUMO- } \\
\text { eIF3i }\end{array}$ & Kan & ColE1 & $18^{\circ} \mathrm{C}$ & $\mathrm{o} / \mathrm{n}$ & Top10F' & 100 & - & - & - & This study \\
\hline JS0010 & $\begin{array}{c}\mathrm{H}_{14} \text {-brSUMO- } \\
\text { eIF3j }\end{array}$ & Kan & ColE1 & $18^{\circ} \mathrm{C}$ & $\mathrm{o} / \mathrm{n}$ & Top10F' & 100 & $50 \mathrm{~T}, 500 \mathrm{~N}, 2 \mathrm{I}, 1 \mathrm{D}$ & $500 \mathrm{I}$ & - & This study \\
\hline JS0011 & $\begin{array}{c}\mathrm{H}_{14} \text {-brSUMO- } \\
\text { eIF3k }\end{array}$ & Kan & ColE1 & $18^{\circ} \mathrm{C}$ & $\mathrm{o} / \mathrm{n}$ & Top10F' & 100 & $50 \mathrm{~T}, 500 \mathrm{~N}, 2 \mathrm{I}, 1 \mathrm{D}$ & $500 \mathrm{I}$ & - & This study \\
\hline JS0012 & $\begin{array}{c}\mathrm{H}_{14} \text {-brSUMO- } \\
\text { eIF3L }\end{array}$ & Kan & ColE1 & $18^{\circ} \mathrm{C}$ & $\mathrm{o} / \mathrm{n}$ & Top10F' & 100 & - & - & - & This study \\
\hline JS0013 & $\begin{array}{c}\mathrm{H}_{14} \text {-brSUMO- } \\
\text { eIF3m }\end{array}$ & Kan & ColE1 & $18^{\circ} \mathrm{C}$ & $\mathrm{o} / \mathrm{n}$ & Top10F' & 100 & - & - & - & This study \\
\hline JS057 & ZZ-TEV-eIF3a & Kan & ColE1 & $18^{\circ} \mathrm{C}$ & $\mathrm{o} / \mathrm{n}$ & Top10F' & 100 & - & - & - & This study \\
\hline
\end{tabular}


Table 4.4-Continued from previous page

\begin{tabular}{|c|c|c|c|c|c|c|c|c|c|c|c|}
\hline \multirow[b]{2}{*}{ Plasmid } & \multirow[b]{2}{*}{ Construct } & \multirow[b]{2}{*}{ Marker } & \multirow[b]{2}{*}{ Ori } & \multicolumn{4}{|c|}{ Expression conditions } & \multicolumn{3}{|c|}{ Purification Conditions } & \multirow[b]{2}{*}{ Reference } \\
\hline & & & & Temp. & Duration & Strain & $\begin{array}{c}\text { IPTG } \\
(\mu \mathbf{M})\end{array}$ & buffer & Elution & $\begin{array}{l}\text { Gel- fil- } \\
\text { tration }\end{array}$ & \\
\hline JS058 & ZZ-TEV-eIF3b & Kan & ColE1 & $18^{\circ} \mathrm{C}$ & $\mathrm{o} / \mathrm{n}$ & Top10F' & 100 & $50 \mathrm{~T}, 500 \mathrm{~N}, 2 \mathrm{I}, 1 \mathrm{D}$ & $500 \mathrm{I}$ & - & This study \\
\hline JS059 & ZZ-TEV-eIF3c & Kan & ColE1 & $18^{\circ} \mathrm{C}$ & $\mathrm{o} / \mathrm{n}$ & Top10F' & 100 & $50 \mathrm{~T}, 500 \mathrm{~N}, 2 \mathrm{I}, 1 \mathrm{D}$ & $500 \mathrm{I}$ & - & This study \\
\hline JS060 & ZZ-TEV-eIF3d & Kan & ColE1 & $18^{\circ} \mathrm{C}$ & $\mathrm{o} / \mathrm{n}$ & Top10F' & 100 & $50 \mathrm{~T}, 500 \mathrm{~N}, 2 \mathrm{I}, 1 \mathrm{D}$ & $500 \mathrm{I}$ & - & This study \\
\hline JS061 & ZZ-TEV-eIF3e & Kan & ColE1 & $18^{\circ} \mathrm{C}$ & $\mathrm{o} / \mathrm{n}$ & Top10F' & 100 & $50 \mathrm{~T}, 500 \mathrm{~N}, 2 \mathrm{I}, 1 \mathrm{D}$ & $500 \mathrm{I}$ & - & This study \\
\hline JS062 & ZZ-TEV-eIF3f & Kan & ColE1 & $18^{\circ} \mathrm{C}$ & $\mathrm{o} / \mathrm{n}$ & Top10F' & 100 & $50 \mathrm{~T}, 500 \mathrm{~N}, 2 \mathrm{I}, 1 \mathrm{D}$ & $500 \mathrm{I}$ & - & This study \\
\hline JS063 & ZZ-TEV-eIF3g & Kan & ColE1 & $18^{\circ} \mathrm{C}$ & $\mathrm{o} / \mathrm{n}$ & Top10F' & 100 & $50 \mathrm{~T}, 500 \mathrm{~N}, 2 \mathrm{I}, 1 \mathrm{D}$ & $500 \mathrm{I}$ & - & This study \\
\hline JS064 & ZZ-TEV-eIF3h & Kan & ColE1 & $18^{\circ} \mathrm{C}$ & $\mathrm{o} / \mathrm{n}$ & Top10F' & 100 & $50 \mathrm{~T}, 500 \mathrm{~N}, 2 \mathrm{I}, 1 \mathrm{D}$ & $500 \mathrm{I}$ & - & This study \\
\hline JS065 & ZZ-TEV-eIF3i & Kan & ColE1 & $18^{\circ} \mathrm{C}$ & $\mathrm{o} / \mathrm{n}$ & Top10F' & 100 & $50 \mathrm{~T}, 500 \mathrm{~N}, 2 \mathrm{I}, 1 \mathrm{D}$ & $500 \mathrm{I}-$ & - & This study \\
\hline JS066 & ZZ-TEV-eIF3j & Kan & ColE1 & $18^{\circ} \mathrm{C}$ & $\mathrm{o} / \mathrm{n}$ & Top10F' & 100 & $50 \mathrm{~T}, 500 \mathrm{~N}, 2 \mathrm{I}, 1 \mathrm{D}$ & $500 \mathrm{I}$ & - & This study \\
\hline JS067 & ZZ-TEV-eIF3k & Kan & ColE1 & $18^{\circ} \mathrm{C}$ & $\mathrm{o} / \mathrm{n}$ & Top10F' & 100 & $50 \mathrm{~T}, 500 \mathrm{~N}, 2 \mathrm{I}, 1 \mathrm{D}$ & $500 \mathrm{I}$ & - & This study \\
\hline JS068 & ZZ-TEV-eIF3L & Kan & ColE1 & $18^{\circ} \mathrm{C}$ & $\mathrm{o} / \mathrm{n}$ & Top10F' & 100 & - & - & - & This study \\
\hline JS069 & ZZ-TEV-eIF3m & Kan & ColE1 & $18^{\circ} \mathrm{C}$ & $\mathrm{o} / \mathrm{n}$ & Top10F' & 100 & - & - & - & This study \\
\hline JS014 & $\begin{array}{c}\mathrm{H}_{10}-\mathrm{ZZ}- \\
\text { TEV-eIF3a }\end{array}$ & Amp & pSC101 & $18^{\circ} \mathrm{C}$ & $\mathrm{o} / \mathrm{n}$ & Top10F' & 100 & - & - & - & This study \\
\hline JS015 & $\begin{array}{c}\mathrm{H}_{10}-\mathrm{ZZ}- \\
\text { TEV-eIF3b }\end{array}$ & Amp & pSC101 & $18^{\circ} \mathrm{C}$ & $\mathrm{o} / \mathrm{n}$ & Top10F' & 100 & $50 \mathrm{~T}, 500 \mathrm{~N}, 2 \mathrm{I}, 1 \mathrm{D}$ & $500 \mathrm{I}$ & - & This study \\
\hline JS016 & $\begin{array}{c}\mathrm{H}_{10}-\mathrm{ZZ}- \\
\text { TEV-eIF3c }\end{array}$ & Amp & pSC101 & $18^{\circ} \mathrm{C}$ & $\mathrm{o} / \mathrm{n}$ & Top10F' & 100 & $\begin{array}{c}-50 \mathrm{~T}, 500 \mathrm{~N} \\
2 \mathrm{I}, 1 \mathrm{D}\end{array}$ & $500 \mathrm{I}$ & - & This study \\
\hline JS017 & $\begin{array}{c}\mathrm{H}_{10}-\mathrm{ZZ}- \\
\text { TEV-eIF3d }\end{array}$ & Amp & pSC101 & $18^{\circ} \mathrm{C}$ & $\mathrm{o} / \mathrm{n}$ & Top10F' & 100 & $50 \mathrm{~T}, 500 \mathrm{~N}, 2 \mathrm{I}, 1 \mathrm{D}$ & $500 \mathrm{I}$ & - & This study \\
\hline JS018 & $\begin{array}{c}\mathrm{H}_{10}-\mathrm{ZZ}- \\
\text { TEV-eIF3e }\end{array}$ & Amp & pSC101 & $18^{\circ} \mathrm{C}$ & $\mathrm{o} / \mathrm{n}$ & Top10F' & 100 & $50 \mathrm{~T}, 500 \mathrm{~N}, 2 \mathrm{I}, 1 \mathrm{D}$ & $500 \mathrm{I}$ & - & This study \\
\hline JS019 & $\begin{array}{c}\mathrm{H}_{10}-\mathrm{ZZ}- \\
\text { TEV-eIF3f }\end{array}$ & Amp & pSC101 & $18^{\circ} \mathrm{C}$ & $\mathrm{o} / \mathrm{n}$ & Top10F' & 100 & $50 \mathrm{~T}, 500 \mathrm{~N}, 2 \mathrm{I}, 1 \mathrm{D}$ & $500 \mathrm{I}$ & - & This study \\
\hline JS020 & $\begin{array}{c}\mathrm{H}_{10}-\mathrm{ZZ}- \\
\text { TEV-eIF3g }\end{array}$ & Amp & pSC101 & $18^{\circ} \mathrm{C}$ & $\mathrm{o} / \mathrm{n}$ & Top10F' & 100 & - & - & - & This study \\
\hline JS021 & $\begin{array}{c}\mathrm{H}_{10}-\mathrm{ZZ}- \\
\text { TEV-eIF3h }\end{array}$ & Amp & pSC101 & $18^{\circ} \mathrm{C}$ & $\mathrm{o} / \mathrm{n}$ & Top10F' & 100 & $50 \mathrm{~T}, 500 \mathrm{~N}, 2 \mathrm{I}, 1 \mathrm{D}$ & $500 \mathrm{I}$ & - & This study \\
\hline
\end{tabular}


Table 4.4-Continued from previous page

\begin{tabular}{|c|c|c|c|c|c|c|c|c|c|c|c|}
\hline \multirow[b]{2}{*}{ Plasmid } & \multirow[b]{2}{*}{ Construct } & \multirow[b]{2}{*}{ Marker } & \multirow[b]{2}{*}{ Ori } & \multicolumn{4}{|c|}{ Expression conditions } & \multicolumn{3}{|c|}{ Purification Conditions } & \multirow[b]{2}{*}{ Reference } \\
\hline & & & & Temp. & Duration & Strain & $\begin{array}{c}\text { IPTG } \\
(\mu \mathbf{M})\end{array}$ & buffer & Elution & $\begin{array}{l}\text { Gel- fil- } \\
\text { tration }\end{array}$ & \\
\hline JS022 & $\begin{array}{c}\mathrm{H}_{10}-\mathrm{ZZ}- \\
\text { TEV-eIF3i }\end{array}$ & Amp & pSC101 & $18^{\circ} \mathrm{C}$ & $\mathrm{o} / \mathrm{n}$ & Top10F' & 100 & - & - & - & This study \\
\hline JS023 & $\begin{array}{c}\mathrm{H}_{10}-\mathrm{ZZ}- \\
\text { TEV-eIF3j }\end{array}$ & Amp & pSC101 & $18^{\circ} \mathrm{C}$ & $\mathrm{o} / \mathrm{n}$ & Top10F' & 100 & $50 \mathrm{~T}, 500 \mathrm{~N}, 2 \mathrm{I}, 1 \mathrm{D}$ & $500 \mathrm{I}$ & - & This study \\
\hline JS024 & $\begin{array}{c}\mathrm{H}_{10}-\mathrm{ZZ}- \\
\text { TEV-eIF3k }\end{array}$ & Amp & pSC101 & $18^{\circ} \mathrm{C}$ & $\mathrm{o} / \mathrm{n}$ & Top10F' & 100 & $50 \mathrm{~T}, 500 \mathrm{~N}, 2 \mathrm{I}, 1 \mathrm{D}$ & $500 \mathrm{I}$ & - & This study \\
\hline JS025 & $\begin{array}{c}\mathrm{H}_{10}-\mathrm{ZZ}- \\
\text { TEV-eIF3L }\end{array}$ & Amp & pSC101 & $18^{\circ} \mathrm{C}$ & $\mathrm{o} / \mathrm{n}$ & Top10F' & 100 & - & - & - & This study \\
\hline JS026 & $\begin{array}{c}\mathrm{H}_{10}-\mathrm{ZZ}- \\
\text { TEV-eIF3m }\end{array}$ & Amp & pSC101 & $18^{\circ} \mathrm{C}$ & $\mathrm{o} / \mathrm{n}$ & Top10F' & 100 & $50 \mathrm{~T}, 500 \mathrm{~N}, 2 \mathrm{I}, 1 \mathrm{D}$ & $500 \mathrm{I}$ & - & This study \\
\hline JS027 & $\begin{array}{c}\mathrm{H}_{14} \text {-shGFP- } \\
\text { brSUMO-eIF3a }\end{array}$ & Kan & ColE1 & $18^{\circ} \mathrm{C}$ & $\mathrm{o} / \mathrm{n}$ & Top10F' & 100 & - & - & - & This study \\
\hline JS028 & $\begin{array}{c}\mathrm{H}_{14} \text {-shGFP- } \\
\text { brSUMO-eIF3b }\end{array}$ & Kan & ColE1 & $18^{\circ} \mathrm{C}$ & $\mathrm{o} / \mathrm{n}$ & Top10F' & 100 & $50 \mathrm{~T}, 500 \mathrm{~N}, 2 \mathrm{I}, 1 \mathrm{D}$ & $500 \mathrm{I}$ & - & This study \\
\hline JS029 & $\begin{array}{c}\mathrm{H}_{14} \text {-shGFP- } \\
\text { brSUMO-eIF3c }\end{array}$ & Kan & ColE1 & $18^{\circ} \mathrm{C}$ & $\mathrm{o} / \mathrm{n}$ & Top10F' & 100 & $50 \mathrm{~T}, 500 \mathrm{~N}, 2 \mathrm{I}, 1 \mathrm{D}$ & $500 \mathrm{I}$ & - & This study \\
\hline JS030 & $\begin{array}{c}\mathrm{H}_{14} \text {-shGFP- } \\
\text { brSUMO-eIF3d }\end{array}$ & Kan & ColE1 & $18^{\circ} \mathrm{C}$ & $\mathrm{o} / \mathrm{n}$ & Top10F' & 100 & $50 \mathrm{~T}, 500 \mathrm{~N}, 2 \mathrm{I}, 1 \mathrm{D}$ & $500 \mathrm{I}$ & - & This study \\
\hline JS031 & $\begin{array}{c}\mathrm{H}_{14} \text {-shGFP- } \\
\text { brSUMO-eIF3e }\end{array}$ & Kan & ColE1 & $18^{\circ} \mathrm{C}$ & $\mathrm{o} / \mathrm{n}$ & Top10F' & 100 & $50 \mathrm{~T}, 500 \mathrm{~N}, 2 \mathrm{I}, 1 \mathrm{D}$ & $500 \mathrm{I}$ & - & This study \\
\hline JS032 & $\begin{array}{c}\mathrm{H}_{14} \text {-shGFP- } \\
\text { brSUMO-eIF3f }\end{array}$ & Kan & ColE1 & $18^{\circ} \mathrm{C}$ & $\mathrm{o} / \mathrm{n}$ & Top10F' & 100 & $50 \mathrm{~T}, 500 \mathrm{~N}, 2 \mathrm{I}, 1 \mathrm{D}$ & $500 \mathrm{I}$ & - & This study \\
\hline JS033 & $\begin{array}{c}\mathrm{H}_{14} \text {-shGFP- } \\
\text { brSUMO-eIF3g }\end{array}$ & Kan & ColE1 & $18^{\circ} \mathrm{C}$ & $\mathrm{o} / \mathrm{n}$ & Top10F' & 100 & $50 \mathrm{~T}, 500 \mathrm{~N}, 2 \mathrm{I}, 1 \mathrm{D}$ & $500 \mathrm{I}$ & - & This study \\
\hline JS034 & $\begin{array}{c}\mathrm{H}_{14} \text {-shGFP- } \\
\text { brSUMO-eIF3h }\end{array}$ & Kan & ColE1 & $18^{\circ} \mathrm{C}$ & $\mathrm{o} / \mathrm{n}$ & Top10F' & 100 & $50 \mathrm{~T}, 500 \mathrm{~N}, 2 \mathrm{I}, 1 \mathrm{D}$ & $500 \mathrm{I}$ & - & This study \\
\hline JS035 & $\begin{array}{c}\mathrm{H}_{14} \text {-shGFP- } \\
\text { brSUMO-eIF3i }\end{array}$ & Kan & ColE1 & $18^{\circ} \mathrm{C}$ & $\mathrm{o} / \mathrm{n}$ & Top10F' & 100 & - & - & - & This study \\
\hline
\end{tabular}


Table 4.4-Continued from previous page

\begin{tabular}{|c|c|c|c|c|c|c|c|c|c|c|c|}
\hline \multirow[b]{2}{*}{ Plasmid } & \multirow[b]{2}{*}{ Construct } & \multirow[b]{2}{*}{ Marker } & \multirow[b]{2}{*}{ Ori } & \multicolumn{4}{|c|}{ Expression conditions } & \multicolumn{3}{|c|}{ Purification Conditions } & \multirow[b]{2}{*}{ Reference } \\
\hline & & & & Temp. & Duration & Strain & $\begin{array}{c}\text { IPTG } \\
(\mu \mathbf{M})\end{array}$ & buffer & Elution & $\begin{array}{l}\text { Gel- fil- } \\
\text { tration }\end{array}$ & \\
\hline JS036 & $\begin{array}{c}\mathrm{H}_{14} \text {-shGFP- } \\
\text { brSUMO-eIF3j }\end{array}$ & Kan & ColE1 & $18^{\circ} \mathrm{C}$ & $\mathrm{o} / \mathrm{n}$ & Top10F' & 100 & $50 \mathrm{~T}, 500 \mathrm{~N}, 2 \mathrm{I}, 1 \mathrm{D}$ & $500 \mathrm{I}$ & - & This study \\
\hline JS037 & $\begin{array}{c}\mathrm{H}_{14} \text {-shGFP- } \\
\text { brSUMO-eIF3k }\end{array}$ & Kan & ColE1 & $18^{\circ} \mathrm{C}$ & $\mathrm{o} / \mathrm{n}$ & Top10F' & 100 & $50 \mathrm{~T}, 500 \mathrm{~N}, 2 \mathrm{I}, 1 \mathrm{D}$ & $500 \mathrm{I}$ & - & This study \\
\hline JS038 & $\begin{array}{c}\mathrm{H}_{14} \text {-shGFP- } \\
\text { brSUMO-eIF3L }\end{array}$ & Kan & ColE1 & $18^{\circ} \mathrm{C}$ & $\mathrm{o} / \mathrm{n}$ & Top10F' & 100 & - & - & - & This study \\
\hline JS039 & $\begin{array}{c}\mathrm{H}_{14} \text {-shGFP- } \\
\text { brSUMO-eIF3m }\end{array}$ & Kan & ColE1 & $18^{\circ} \mathrm{C}$ & $\mathrm{o} / \mathrm{n}$ & Top10F' & 100 & $50 \mathrm{~T}, 500 \mathrm{~N}, 2 \mathrm{I}, 1 \mathrm{D}$ & $500 \mathrm{I}$ & - & This study \\
\hline JS070 & $\begin{array}{l}\mathrm{H}_{14} \text {-MBP- } \\
\text { TEV-eIF3a }\end{array}$ & Spec & $\mathrm{p} 15 \mathrm{~A}$ & $18^{\circ} \mathrm{C}$ & $\mathrm{o} / \mathrm{n}$ & Top10F' & 100 & $50 \mathrm{~T}, 500 \mathrm{~N}, 2 \mathrm{I}, 1 \mathrm{D}$ & $500 \mathrm{I}$ & - & This study \\
\hline JS071 & $\begin{array}{l}\mathrm{H}_{14} \text {-MBP- } \\
\text { TEV-eIF3b }\end{array}$ & Spec & $\mathrm{p} 15 \mathrm{~A}$ & $18^{\circ} \mathrm{C}$ & $\mathrm{o} / \mathrm{n}$ & Top10F' & 100 & $50 \mathrm{~T}, 500 \mathrm{~N}, 2 \mathrm{I}, 1 \mathrm{D}$ & $500 \mathrm{I}$ & - & This study \\
\hline JS072 & $\begin{array}{l}\mathrm{H}_{14} \text {-MBP- } \\
\text { TEV-eIF3c }\end{array}$ & Spec & p15A & $18^{\circ} \mathrm{C}$ & $\mathrm{o} / \mathrm{n}$ & Top10F' & 100 & $50 \mathrm{~T}, 500 \mathrm{~N}, 2 \mathrm{I}, 1 \mathrm{D}$ & $500 \mathrm{I}$ & - & This study \\
\hline JS073 & $\begin{array}{l}\mathrm{H}_{14} \text {-MBP- } \\
\text { TEV-eIF3d }\end{array}$ & Spec & $\mathrm{p} 15 \mathrm{~A}$ & $18^{\circ} \mathrm{C}$ & $\mathrm{o} / \mathrm{n}$ & Top10F' & 100 & $50 \mathrm{~T}, 500 \mathrm{~N}, 2 \mathrm{I}, 1 \mathrm{D}$ & $500 \mathrm{I}$ & - & This study \\
\hline JS074 & $\begin{array}{l}\mathrm{H}_{14} \text {-MBP- } \\
\text { TEV-eIF3e }\end{array}$ & Spec & $\mathrm{p} 15 \mathrm{~A}$ & $18^{\circ} \mathrm{C}$ & $\mathrm{o} / \mathrm{n}$ & Top10F' & 100 & $50 \mathrm{~T}, 500 \mathrm{~N}, 2 \mathrm{I}, 1 \mathrm{D}$ & $500 \mathrm{I}$ & - & This study \\
\hline JS075 & $\begin{array}{l}\mathrm{H}_{14} \text {-MBP- } \\
\text { TEV-eIF3f }\end{array}$ & Spec & $\mathrm{p} 15 \mathrm{~A}$ & $18^{\circ} \mathrm{C}$ & $\mathrm{o} / \mathrm{n}$ & Top10F' & 100 & $50 \mathrm{~T}, 500 \mathrm{~N}, 2 \mathrm{I}, 1 \mathrm{D}$ & $500 \mathrm{I}$ & - & This study \\
\hline JS076 & $\begin{array}{l}\mathrm{H}_{14} \text {-MBP- } \\
\text { TEV-eIF3g }\end{array}$ & Spec & $\mathrm{p} 15 \mathrm{~A}$ & $18^{\circ} \mathrm{C}$ & $\mathrm{o} / \mathrm{n}$ & Top10F' & 100 & $50 \mathrm{~T}, 500 \mathrm{~N}, 2 \mathrm{I}, 1 \mathrm{D}$ & $500 \mathrm{I}$ & - & This study \\
\hline JS077 & $\begin{array}{l}\mathrm{H}_{14} \text {-MBP- } \\
\text { TEV-eIF3h }\end{array}$ & Spec & $\mathrm{p} 15 \mathrm{~A}$ & $18^{\circ} \mathrm{C}$ & $\mathrm{o} / \mathrm{n}$ & Top10F' & 100 & $50 \mathrm{~T}, 500 \mathrm{~N}, 2 \mathrm{I}, 1 \mathrm{D}$ & $500 \mathrm{I}$ & - & This study \\
\hline JS078 & $\begin{array}{l}\mathrm{H}_{14}-\mathrm{MBP}- \\
\text { TEV-eIF3i }\end{array}$ & Spec & $\mathrm{p} 15 \mathrm{~A}$ & $18^{\circ} \mathrm{C}$ & $\mathrm{o} / \mathrm{n}$ & Top10F' & 100 & $50 \mathrm{~T}, 500 \mathrm{~N}, 2 \mathrm{I}, 1 \mathrm{D}$ & $500 \mathrm{I}$ & - & This study \\
\hline JS079 & $\begin{array}{l}\mathrm{H}_{14} \text {-MBP- } \\
\text { TEV-eIF3j }\end{array}$ & Spec & $\mathrm{p} 15 \mathrm{~A}$ & $18^{\circ} \mathrm{C}$ & $\mathrm{o} / \mathrm{n}$ & Top10F' & 100 & $50 \mathrm{~T}, 500 \mathrm{~N}, 2 \mathrm{I}, 1 \mathrm{D}$ & $500 \mathrm{I}$ & - & This study \\
\hline
\end{tabular}


Table 4.4-Continued from previous page

\begin{tabular}{|c|c|c|c|c|c|c|c|c|c|c|c|}
\hline \multirow[b]{2}{*}{ Plasmid } & \multirow[b]{2}{*}{ Construct } & \multirow[b]{2}{*}{ Marker } & \multirow[b]{2}{*}{ Ori } & \multicolumn{4}{|c|}{ Expression conditions } & \multicolumn{3}{|c|}{ Purification Conditions } & \multirow[b]{2}{*}{ Reference } \\
\hline & & & & Temp. & Duration & Strain & $\begin{array}{c}\text { IPTG } \\
(\mu \mathbf{M})\end{array}$ & buffer & Elution & $\begin{array}{l}\text { Gel- fil- } \\
\text { tration }\end{array}$ & \\
\hline JS080 & $\begin{array}{l}\mathrm{H}_{14} \text {-MBP- } \\
\text { TEV-eIF3k }\end{array}$ & Spec & $\mathrm{p} 15 \mathrm{~A}$ & $18^{\circ} \mathrm{C}$ & $\mathrm{o} / \mathrm{n}$ & Top10F' & 100 & $50 \mathrm{~T}, 500 \mathrm{~N}, 2 \mathrm{I}, 1 \mathrm{D}$ & $500 \mathrm{I}$ & - & This study \\
\hline JS081 & $\begin{array}{l}\mathrm{H}_{14} \text {-MBP- } \\
\text { TEV-eIF3L }\end{array}$ & Spec & $\mathrm{p} 15 \mathrm{~A}$ & $18^{\circ} \mathrm{C}$ & $\mathrm{o} / \mathrm{n}$ & Top10F' & 100 & $50 \mathrm{~T}, 500 \mathrm{~N}, 2 \mathrm{I}, 1 \mathrm{D}$ & $500 \mathrm{I}$ & - & This study \\
\hline JS082 & $\begin{array}{l}\mathrm{H}_{14} \text {-MBP- } \\
\text { TEV-eIF3m }\end{array}$ & Spec & $\mathrm{p} 15 \mathrm{~A}$ & $18^{\circ} \mathrm{C}$ & $\mathrm{o} / \mathrm{n}$ & Top10F' & 100 & $50 \mathrm{~T}, 500 \mathrm{~N}, 2 \mathrm{I}, 1 \mathrm{D}$ & $500 \mathrm{I}$ & - & This study \\
\hline JS145 & $\begin{array}{c}\mathrm{H}_{14} \text {-MBP- } \\
\text { brSUMO-eIF3a }\end{array}$ & Kan & ColE1 & $18^{\circ} \mathrm{C}$ & $\mathrm{o} / \mathrm{n}$ & $\begin{array}{c}\text { NEB } \\
\text { Express }\end{array}$ & 100 & $50 \mathrm{~T}, 300 \mathrm{~N}, 2 \mathrm{I}, 1 \mathrm{D}$ & Protease & - & This study \\
\hline JS146 & $\begin{array}{c}\mathrm{H}_{14}-\mathrm{MBP}- \\
\text { brSUMO-eIF3b }\end{array}$ & Kan & ColE1 & $18^{\circ} \mathrm{C}$ & $\mathrm{o} / \mathrm{n}$ & $\begin{array}{c}\text { NEB } \\
\text { Express }\end{array}$ & 100 & $50 \mathrm{~T}, 300 \mathrm{~N}, 2 \mathrm{I}, 1 \mathrm{D}$ & Protease & - & This study \\
\hline JS147 & $\begin{array}{c}\mathrm{H}_{14-\mathrm{MBP}-} \\
\text { brSUMO-eIF3c }\end{array}$ & Kan & ColE1 & $18^{\circ} \mathrm{C}$ & $\mathrm{o} / \mathrm{n}$ & $\begin{array}{c}\text { NEB } \\
\text { Express }\end{array}$ & 100 & $50 \mathrm{~T}, 300 \mathrm{~N}, 2 \mathrm{I}, 1 \mathrm{D}$ & Protease & - & This study \\
\hline JS148 & $\begin{array}{c}\mathrm{H}_{14} \text {-MBP- } \\
\text { brSUMO-eIF3d }\end{array}$ & Kan & ColE1 & $18^{\circ} \mathrm{C}$ & $\mathrm{o} / \mathrm{n}$ & $\begin{array}{c}\text { NEB } \\
\text { Express }\end{array}$ & 100 & $50 \mathrm{~T}, 300 \mathrm{~N}, 2 \mathrm{I}, 1 \mathrm{D}$ & Protease & - & This study \\
\hline JS149 & $\begin{array}{c}\mathrm{H}_{14} \text {-MBP- } \\
\text { brSUMO-eIF3e }\end{array}$ & Kan & ColE1 & $18^{\circ} \mathrm{C}$ & $\mathrm{o} / \mathrm{n}$ & $\begin{array}{c}\text { NEB } \\
\text { Express }\end{array}$ & 100 & $50 \mathrm{~T}, 300 \mathrm{~N}, 2 \mathrm{I}, 1 \mathrm{D}$ & Protease & - & This study \\
\hline JS150 & $\begin{array}{c}\mathrm{H}_{14} \text {-MBP- } \\
\text { brSUMO-eIF3f }\end{array}$ & Kan & ColE1 & $18^{\circ} \mathrm{C}$ & $\mathrm{o} / \mathrm{n}$ & $\begin{array}{c}\text { NEB } \\
\text { Express }\end{array}$ & 100 & $50 \mathrm{~T}, 300 \mathrm{~N}, 2 \mathrm{I}, 1 \mathrm{D}$ & Protease & - & This study \\
\hline JS151 & $\begin{array}{c}\mathrm{H}_{14} \text {-MBP- } \\
\text { brSUMO-eIF3g }\end{array}$ & Kan & ColE1 & $18^{\circ} \mathrm{C}$ & $\mathrm{o} / \mathrm{n}$ & $\begin{array}{c}\text { NEB } \\
\text { Express }\end{array}$ & 100 & $50 \mathrm{~T}, 300 \mathrm{~N}, 2 \mathrm{I}, 1 \mathrm{D}$ & Protease & - & This study \\
\hline JS152 & $\begin{array}{c}\mathrm{H}_{14} \text {-MBP- } \\
\text { brSUMO-eIF3h }\end{array}$ & Kan & ColE1 & $18^{\circ} \mathrm{C}$ & $\mathrm{o} / \mathrm{n}$ & $\begin{array}{c}\text { NEB } \\
\text { Express }\end{array}$ & 100 & $50 \mathrm{~T}, 300 \mathrm{~N}, 2 \mathrm{I}, 1 \mathrm{D}$ & Protease & - & This study \\
\hline JS153 & $\begin{array}{c}\mathrm{H}_{14} \text {-MBP- } \\
\text { brSUMO-eIF3i }\end{array}$ & Kan & ColE1 & $18^{\circ} \mathrm{C}$ & $\mathrm{o} / \mathrm{n}$ & $\begin{array}{c}\text { NEB } \\
\text { Express }\end{array}$ & 100 & $50 \mathrm{~T}, 300 \mathrm{~N}, 2 \mathrm{I}, 1 \mathrm{D}$ & Protease & - & This study \\
\hline JS154 & $\begin{array}{c}\mathrm{H}_{14} \text {-MBP- } \\
\text { brSUMO-eIF3j }\end{array}$ & Kan & ColE1 & $18^{\circ} \mathrm{C}$ & $\mathrm{o} / \mathrm{n}$ & $\begin{array}{c}\text { NEB } \\
\text { Express }\end{array}$ & 100 & $50 \mathrm{~T}, 300 \mathrm{~N}, 2 \mathrm{I}, 1 \mathrm{D}$ & Protease & - & This study \\
\hline JS155 & $\begin{array}{c}\mathrm{H}_{14} \text {-MBP- } \\
\text { brSUMO-eIF3k }\end{array}$ & Kan & ColE1 & $18^{\circ} \mathrm{C}$ & $\mathrm{o} / \mathrm{n}$ & $\begin{array}{c}\text { NEB } \\
\text { Express }\end{array}$ & 100 & $50 \mathrm{~T}, 300 \mathrm{~N}, 2 \mathrm{I}, 1 \mathrm{D}$ & Protease & - & This study \\
\hline
\end{tabular}


Table 4.4-Continued from previous page

\begin{tabular}{|c|c|c|c|c|c|c|c|c|c|c|c|}
\hline \multirow[b]{2}{*}{ Plasmid } & \multirow[b]{2}{*}{ Construct } & \multirow[b]{2}{*}{ Marker } & \multirow[b]{2}{*}{ Ori } & \multicolumn{4}{|c|}{ Expression conditions } & \multicolumn{3}{|c|}{ Purification Conditions } & \multirow[b]{2}{*}{ Reference } \\
\hline & & & & Temp. & Duration & Strain & $\begin{array}{c}\text { IPTG } \\
(\mu \mathrm{M})\end{array}$ & buffer & Elution & $\begin{array}{l}\text { Gel- fil- } \\
\text { tration }\end{array}$ & \\
\hline JS156 & $\begin{array}{c}\mathrm{H}_{14} \text {-MBP- } \\
\text { brSUMO-eIF3L }\end{array}$ & Kan & ColE1 & $18^{\circ} \mathrm{C}$ & $\mathrm{o} / \mathrm{n}$ & $\begin{array}{c}\text { NEB } \\
\text { Express }\end{array}$ & 100 & $50 \mathrm{~T}, 300 \mathrm{~N}, 2 \mathrm{I}, 1 \mathrm{D}$ & Protease & - & This study \\
\hline JS157 & $\begin{array}{c}\mathrm{H}_{14} \text {-MBP- } \\
\text { brSUMO-eIF3m }\end{array}$ & Kan & ColE1 & $18^{\circ} \mathrm{C}$ & $\mathrm{o} / \mathrm{n}$ & $\begin{array}{c}\text { NEB } \\
\text { Express }\end{array}$ & 100 & $50 \mathrm{~T}, 300 \mathrm{~N}, 2 \mathrm{I}, 1 \mathrm{D}$ & Protease & - & This study \\
\hline JS228 & $\begin{array}{c}\mathrm{H}_{14} \text {-MBP- } \\
\text { brSUMO-eIF1 }\end{array}$ & Kan & ColE1 & $18^{\circ} \mathrm{C}$ & $\mathrm{o} / \mathrm{n}$ & $\begin{array}{c}\text { NEB } \\
\text { Express }\end{array}$ & 100 & $50 \mathrm{~T}, 300 \mathrm{~N}, 2 \mathrm{I}, 1 \mathrm{D}$ & Protease & - & This study \\
\hline JS229 & $\begin{array}{c}\mathrm{H}_{14} \text {-MBP- } \\
\text { brSUMO-eIF1A }\end{array}$ & Kan & ColE1 & $18^{\circ} \mathrm{C}$ & $\mathrm{o} / \mathrm{n}$ & $\begin{array}{c}\text { NEB } \\
\text { Express }\end{array}$ & 100 & $50 \mathrm{~T}, 300 \mathrm{~N}, 2 \mathrm{I}, 1 \mathrm{D}$ & Protease & - & This study \\
\hline JS230 & $\begin{array}{c}\mathrm{H}_{14} \text {-MBP- } \\
\text { brSUMO-eIF } 2 \alpha\end{array}$ & Kan & ColE1 & $18^{\circ} \mathrm{C}$ & $\mathrm{o} / \mathrm{n}$ & $\begin{array}{c}\text { NEB } \\
\text { Express }\end{array}$ & 100 & $50 \mathrm{~T}, 300 \mathrm{~N}, 2 \mathrm{I}, 1 \mathrm{D}$ & Protease & - & This study \\
\hline JS231 & $\begin{array}{c}\mathrm{H}_{14}-\mathrm{MBP}- \\
\text { brSUMO-eIF } 2 \gamma\end{array}$ & Kan & ColE1 & $18^{\circ} \mathrm{C}$ & $\mathrm{o} / \mathrm{n}$ & $\begin{array}{c}\text { NEB } \\
\text { Express }\end{array}$ & 100 & $50 \mathrm{~T}, 300 \mathrm{~N}, 2 \mathrm{I}, 1 \mathrm{D}$ & Protease & - & This study \\
\hline JS232 & $\begin{array}{c}\mathrm{H}_{14} \text {-MBP- } \\
\text { brSUMO-eIF2Bß }\end{array}$ & Kan & ColE1 & $18^{\circ} \mathrm{C}$ & $\mathrm{o} / \mathrm{n}$ & $\begin{array}{c}\text { NEB } \\
\text { Express }\end{array}$ & 100 & $50 \mathrm{~T}, 300 \mathrm{~N}, 2 \mathrm{I}, 1 \mathrm{D}$ & Protease & - & This study \\
\hline JS233 & $\begin{array}{c}\mathrm{H}_{14} \text {-MBP- } \\
\text { brSUMO-eIF2B } \gamma\end{array}$ & Kan & ColE1 & $18^{\circ} \mathrm{C}$ & $\mathrm{o} / \mathrm{n}$ & $\begin{array}{c}\text { NEB } \\
\text { Express }\end{array}$ & 100 & $50 \mathrm{~T}, 300 \mathrm{~N}, 2 \mathrm{I}, 1 \mathrm{D}$ & Protease & - & This study \\
\hline JS234 & $\begin{array}{c}\mathrm{H}_{14} \text {-MBP- } \\
\text { brSUMO-eIF2B } \epsilon\end{array}$ & Kan & ColE1 & $18^{\circ} \mathrm{C}$ & $\mathrm{o} / \mathrm{n}$ & $\begin{array}{c}\text { NEB } \\
\text { Express }\end{array}$ & 100 & $50 \mathrm{~T}, 300 \mathrm{~N}, 2 \mathrm{I}, 1 \mathrm{D}$ & Protease & - & This study \\
\hline JS235 & $\begin{array}{c}\mathrm{H}_{14} \text {-MBP- } \\
\text { brSUMO-eIF4A }\end{array}$ & Kan & ColE1 & $18^{\circ} \mathrm{C}$ & $\mathrm{o} / \mathrm{n}$ & $\begin{array}{c}\text { NEB } \\
\text { Express }\end{array}$ & 100 & $50 \mathrm{~T}, 300 \mathrm{~N}, 2 \mathrm{I}, 1 \mathrm{D}$ & Protease & - & This study \\
\hline JS236 & $\begin{array}{c}\mathrm{H}_{14} \text {-MBP- } \\
\text { brSUMO-eIF4B }\end{array}$ & Kan & ColE1 & $18^{\circ} \mathrm{C}$ & $\mathrm{o} / \mathrm{n}$ & $\begin{array}{c}\text { NEB } \\
\text { Express }\end{array}$ & 100 & $50 \mathrm{~T}, 300 \mathrm{~N}, 2 \mathrm{I}, 1 \mathrm{D}$ & Protease & - & This study \\
\hline JS237 & $\begin{array}{c}\mathrm{H}_{14} \text {-MBP- } \\
\text { brSUMO- } \\
\text { eIF4isoE }\end{array}$ & Kan & ColE1 & $18^{\circ} \mathrm{C}$ & $\mathrm{o} / \mathrm{n}$ & $\begin{array}{c}\text { NEB } \\
\text { Express }\end{array}$ & 100 & $50 \mathrm{~T}, 300 \mathrm{~N}, 2 \mathrm{I}, 1 \mathrm{D}$ & Protease & - & This study \\
\hline JS238 & $\begin{array}{c}\mathrm{H}_{14} \text {-MBP- } \\
\text { brSUMO-eIF4E }\end{array}$ & Kan & ColE1 & $18^{\circ} \mathrm{C}$ & $\mathrm{o} / \mathrm{n}$ & $\begin{array}{c}\text { NEB } \\
\text { Express }\end{array}$ & 100 & $50 \mathrm{~T}, 300 \mathrm{~N}, 2 \mathrm{I}, 1 \mathrm{D}$ & Protease & - & This study \\
\hline
\end{tabular}


Table 4.4-Continued from previous page

\begin{tabular}{|c|c|c|c|c|c|c|c|c|c|c|c|}
\hline \multirow[b]{2}{*}{ Plasmid } & \multirow[b]{2}{*}{ Construct } & \multirow[b]{2}{*}{ Marker } & \multirow[b]{2}{*}{ Ori } & \multicolumn{4}{|c|}{ Expression conditions } & \multicolumn{3}{|c|}{ Purification Conditions } & \multirow[b]{2}{*}{ Reference } \\
\hline & & & & Temp. & Duration & Strain & $\begin{array}{c}\text { IPTG } \\
(\mu \mathrm{M})\end{array}$ & buffer & Elution & $\begin{array}{l}\text { Gel- fil- } \\
\text { tration }\end{array}$ & \\
\hline JS239 & $\begin{array}{c}\mathrm{H}_{14} \text {-MBP- } \\
\text { brSUMO-eIF4G } \\
\text { (c-terminal) }\end{array}$ & Kan & ColE1 & $18^{\circ} \mathrm{C}$ & $\mathrm{o} / \mathrm{n}$ & $\begin{array}{c}\text { NEB } \\
\text { Express }\end{array}$ & 100 & $50 \mathrm{~T}, 300 \mathrm{~N}, 2 \mathrm{I}, 1 \mathrm{D}$ & Protease & - & This study \\
\hline JS240 & $\begin{array}{c}\mathrm{H}_{14} \text {-MBP- } \\
\text { brSUMO-eIF4G } \\
\text { (n-terminal) }\end{array}$ & Kan & ColE1 & $18^{\circ} \mathrm{C}$ & $\mathrm{o} / \mathrm{n}$ & $\begin{array}{c}\text { NEB } \\
\text { Express }\end{array}$ & 100 & $50 \mathrm{~T}, 300 \mathrm{~N}, 2 \mathrm{I}, 1 \mathrm{D}$ & Protease & - & This study \\
\hline JS241 & $\begin{array}{c}\mathrm{H}_{14} \text {-MBP- } \\
\text { brSUMO-eIF5A }\end{array}$ & Kan & ColE1 & $18^{\circ} \mathrm{C}$ & $\mathrm{o} / \mathrm{n}$ & $\begin{array}{c}\text { NEB } \\
\text { Express }\end{array}$ & 100 & $50 \mathrm{~T}, 300 \mathrm{~N}, 2 \mathrm{I}, 1 \mathrm{D}$ & Protease & - & This study \\
\hline JS242 & $\begin{array}{c}\mathrm{H}_{14}-\mathrm{MBP}- \\
\text { brSUMO-eIF6 }\end{array}$ & Kan & ColE1 & $18^{\circ} \mathrm{C}$ & $\mathrm{o} / \mathrm{n}$ & $\begin{array}{c}\text { NEB } \\
\text { Express }\end{array}$ & 100 & $50 \mathrm{~T}, 300 \mathrm{~N}, 2 \mathrm{I}, 1 \mathrm{D}$ & Protease & - & This study \\
\hline JS243 & $\begin{array}{c}\mathrm{H}_{14-\mathrm{MBP}}- \\
\text { brSUMO-nCBP }\end{array}$ & Kan & ColE1 & $18^{\circ} \mathrm{C}$ & $\mathrm{o} / \mathrm{n}$ & $\begin{array}{c}\text { NEB } \\
\text { Express }\end{array}$ & 100 & $50 \mathrm{~T}, 300 \mathrm{~N}, 2 \mathrm{I}, 1 \mathrm{D}$ & Protease & - & This study \\
\hline JS244 & $\begin{array}{c}\mathrm{H}_{14}-\mathrm{MBP}- \\
\text { brSUMO-eEF1 } \alpha\end{array}$ & Kan & ColE1 & $18^{\circ} \mathrm{C}$ & $\mathrm{o} / \mathrm{n}$ & $\begin{array}{c}\text { NEB } \\
\text { Express }\end{array}$ & 100 & $50 \mathrm{~T}, 300 \mathrm{~N}, 2 \mathrm{I}, 1 \mathrm{D}$ & Protease & - & This study \\
\hline $\mathrm{JS} 245$ & $\begin{array}{c}\mathrm{H}_{14} \text {-MBP- } \\
\text { brSUMO-eEF1B } \alpha\end{array}$ & Kan & ColE1 & $18^{\circ} \mathrm{C}$ & $\mathrm{o} / \mathrm{n}$ & $\begin{array}{c}\text { NEB } \\
\text { Express }\end{array}$ & 100 & $50 \mathrm{~T}, 300 \mathrm{~N}, 2 \mathrm{I}, 1 \mathrm{D}$ & Protease & - & This study \\
\hline JS246 & $\begin{array}{c}\mathrm{H}_{14} \text {-MBP- } \\
\text { brSUMO-eIF5 }\end{array}$ & Kan & ColE1 & $18^{\circ} \mathrm{C}$ & $\mathrm{o} / \mathrm{n}$ & $\begin{array}{c}\text { NEB } \\
\text { Express }\end{array}$ & 100 & $50 \mathrm{~T}, 300 \mathrm{~N}, 2 \mathrm{I}, 1 \mathrm{D}$ & Protease & - & This study \\
\hline JS247 & $\begin{array}{c}\mathrm{H}_{14} \text {-MBP- } \\
\text { brSUMO-eIF2B } \alpha\end{array}$ & Kan & ColE1 & $18^{\circ} \mathrm{C}$ & $\mathrm{o} / \mathrm{n}$ & $\begin{array}{c}\text { NEB } \\
\text { Express }\end{array}$ & 100 & $50 \mathrm{~T}, 300 \mathrm{~N}, 2 \mathrm{I}, 1 \mathrm{D}$ & Protease & - & This study \\
\hline JS248 & $\begin{array}{c}\mathrm{H}_{14}-\mathrm{MBP}- \\
\text { brSUMO-eIF2B } \epsilon \\
\text { (cat. domain) }\end{array}$ & Kan & ColE1 & $18^{\circ} \mathrm{C}$ & $\mathrm{o} / \mathrm{n}$ & $\begin{array}{c}\text { NEB } \\
\text { Express }\end{array}$ & 100 & $50 \mathrm{~T}, 300 \mathrm{~N}, 2 \mathrm{I}, 1 \mathrm{D}$ & Protease & - & This study \\
\hline JS249 & $\begin{array}{c}\mathrm{H}_{14} \text {-MBP- } \\
\text { brSUMO-eIF2B } \delta\end{array}$ & Kan & ColE1 & $18^{\circ} \mathrm{C}$ & $\mathrm{o} / \mathrm{n}$ & $\begin{array}{c}\text { NEB } \\
\text { Express }\end{array}$ & 100 & $50 \mathrm{~T}, 300 \mathrm{~N}, 2 \mathrm{I}, 1 \mathrm{D}$ & Protease & - & This study \\
\hline JS252 & $\begin{array}{c}\mathrm{H}_{14-\mathrm{MBP}-} \\
\text { brSUMO-eIF2ß }\end{array}$ & Kan & ColE1 & $18^{\circ} \mathrm{C}$ & $\mathrm{o} / \mathrm{n}$ & $\begin{array}{c}\text { NEB } \\
\text { Express }\end{array}$ & 100 & $50 \mathrm{~T}, 300 \mathrm{~N}, 2 \mathrm{I}, 1 \mathrm{D}$ & Protease & - & This study \\
\hline
\end{tabular}


Table 4.4-Continued from previous page

\begin{tabular}{|c|c|c|c|c|c|c|c|c|c|c|c|}
\hline \multirow[b]{2}{*}{ Plasmid } & \multirow[b]{2}{*}{ Construct } & \multirow[b]{2}{*}{ Marker } & \multirow[b]{2}{*}{ Ori } & \multicolumn{4}{|c|}{ Expression conditions } & \multicolumn{3}{|c|}{ Purification Conditions } & \multirow[b]{2}{*}{ Reference } \\
\hline & & & & Temp. & Duration & Strain & $\begin{array}{c}\text { IPTG } \\
(\mu \mathrm{M})\end{array}$ & buffer & Elution & $\begin{array}{l}\text { Gel- fil- } \\
\text { tration }\end{array}$ & \\
\hline JS253 & $\begin{array}{c}\mathrm{H}_{14} \text {-MBP- } \\
\text { brSUMO-PABP }\end{array}$ & Kan & ColE1 & $18^{\circ} \mathrm{C}$ & $\mathrm{o} / \mathrm{n}$ & $\begin{array}{c}\text { NEB } \\
\text { Express }\end{array}$ & 100 & $50 \mathrm{~T}, 300 \mathrm{~N}, 2 \mathrm{I}, 1 \mathrm{D}$ & Protease & - & This study \\
\hline JS218 & $\begin{array}{l}\mathrm{H}_{14} \text {-Avi-TEV- } \\
\text { mm Exportin4 }\end{array}$ & Kan & ColE1 & $25^{\circ} \mathrm{C}$ & $6 \mathrm{~h}$ & $\begin{array}{c}\text { NEB } \\
\text { Express }\end{array}$ & 200 & $50 \mathrm{~T}, 300 \mathrm{~N}, 2 \mathrm{I}, 1 \mathrm{D}$ & $300 \mathrm{I}$ & - & This study \\
\hline JS227 & $\begin{array}{c}\mathrm{H}_{14} \text {-MBP- } \\
\text { brSUMO-mm } \\
\text { Exportin4 }\end{array}$ & Kan & ColE1 & $25^{\circ} \mathrm{C}$ & $6 \mathrm{~h}$ & $\begin{array}{c}\text { NEB } \\
\text { Express }\end{array}$ & 200 & $50 \mathrm{~T}, 300 \mathrm{~N}, 2 \mathrm{I}, 1 \mathrm{D}$ & $300 \mathrm{I}$ & - & This study \\
\hline pKG029 & $\begin{array}{c}\mathrm{H}_{14-\mathrm{ZZ}} \\
\text { SUMOStar- } \\
\text { RanQ69L }_{(5-180)} \\
\end{array}$ & Kan & ColE1 & $18^{\circ} \mathrm{C}$ & $\mathrm{o} / \mathrm{n}$ & $\begin{array}{c}\text { NEB } \\
\text { Express }\end{array}$ & 100 & $50 \mathrm{H}, 500 \mathrm{~N}, 2 \mathrm{M}$ & $500 \mathrm{I}$ & + & $\begin{array}{c}\text { K.Gencalp } \\
\text { (unpublished) }\end{array}$ \\
\hline $\mathrm{CHC} 01$ & $\begin{array}{c}\mathrm{H}_{14} \text {-brSUMO- } \\
\text { Crm1 G502A }\end{array}$ & Kan & ColE1 & $18^{\circ} \mathrm{C}$ & $\mathrm{o} / \mathrm{n}$ & $\begin{array}{c}\text { NEB } \\
\text { Express }\end{array}$ & 200 & $50 \mathrm{~T}, 200 \mathrm{~N}, 2 \mathrm{I}, 1 \mathrm{D}$ & Protease & - & $\begin{array}{c}\text { H.Chug } \\
\text { (unpublished) }\end{array}$ \\
\hline pKKNES100 & $\begin{array}{c}\mathrm{H}_{14} \text {-ZZ-brSUMO- } \\
\mathrm{mm} \text { Crm1 }\end{array}$ & Kan & ColE1 & $18^{\circ} \mathrm{C}$ & $\mathrm{o} / \mathrm{n}$ & $\begin{array}{c}\text { NEB } \\
\text { Express }\end{array}$ & 200 & $50 \mathrm{~T}, 200 \mathrm{~N}, 2 \mathrm{I}, 1 \mathrm{D}$ & Protease & - & $\begin{array}{c}\text { K.Kirli } \\
\text { (unpublished) }\end{array}$ \\
\hline pKG061 & $\begin{array}{c}\mathrm{H}_{14} \text {-brSUMO- } \\
\text { hs Xpo6 }\end{array}$ & Kan & ColE1 & \multicolumn{7}{|c|}{ provided by K.Gencalp } & $\begin{array}{c}\text { K.Gencalp } \\
\text { (unpublished) }\end{array}$ \\
\hline pDG81 & $\mathrm{H}_{14}$-TEV-CAS & Kan & ColE1 & $25^{\circ} \mathrm{C}$ & $6 \mathrm{~h}$ & $\begin{array}{c}\text { NEB } \\
\text { Express }\end{array}$ & 200 & $50 \mathrm{~T}, 200 \mathrm{~N}, 2 \mathrm{I}, 1 \mathrm{D}$ & $300 \mathrm{I}$ & - & $\begin{array}{c}\text { D. Görlich } \\
\text { (unpublished) }\end{array}$ \\
\hline pTGA34 & $\begin{array}{c}\mathrm{H}_{10}-\mathrm{ZZ}-\mathrm{TEV}-\mathrm{NS} 2 \\
\text { peptide }\end{array}$ & Kan & ColE1 & $30^{\circ} \mathrm{C}$ & $6 \mathrm{~h}$ & $\begin{array}{c}\text { NEB } \\
\text { Express }\end{array}$ & 200 & $50 \mathrm{~T}, 200 \mathrm{~N}, 2 \mathrm{I}, 1 \mathrm{D}$ & $300 \mathrm{I}$ & - & $\begin{array}{c}\text { Güttler } \\
\text { et al. }(2010) \\
\end{array}$ \\
\hline pTGA283 & $\begin{array}{l}\mathrm{H}_{10} \text {-ZZ-TEV- } \\
\text { PKI-02c-SPN1 }\end{array}$ & Kan & ColE1 & \multicolumn{7}{|c|}{ provided by T.Güttler } & $\begin{array}{l}\text { Mönecke } \\
\text { et al. (2009) }\end{array}$ \\
\hline pTGA344 & $\begin{array}{l}\mathrm{H}_{10}-\mathrm{ZZ}-\mathrm{TEV}- \\
\text { REV5N-SPN1 }\end{array}$ & Kan & ColE1 & \multicolumn{7}{|c|}{ provided by T.Güttler } & $\begin{array}{l}\text { Mönecke } \\
\text { et al. (2009) }\end{array}$ \\
\hline $\mathrm{CHC} 04$ & $\begin{array}{l}\mathrm{H}_{14} \text {-brSUMO- } \\
\text { Crm1 A541R }\end{array}$ & Kan & ColE1 & $18^{\circ} \mathrm{C}$ & $\mathrm{o} / \mathrm{n}$ & $\begin{array}{c}\text { NEB } \\
\text { Express }\end{array}$ & 200 & $50 \mathrm{~T}, 200 \mathrm{~N}, 2 \mathrm{I}, 1 \mathrm{D}$ & Protease & - & $\begin{array}{c}\text { H.Chug } \\
\text { (unpublished) }\end{array}$ \\
\hline CHC11 & $\begin{array}{c}\mathrm{H}_{14} \text {-brSUMO- } \\
\text { Crm1 K568E }\end{array}$ & Kan & ColE1 & $18^{\circ} \mathrm{C}$ & $\mathrm{o} / \mathrm{n}$ & $\begin{array}{c}\text { NEB } \\
\text { Express }\end{array}$ & 200 & $50 \mathrm{~T}, 200 \mathrm{~N}, 2 \mathrm{I}, 1 \mathrm{D}$ & Protease & - & $\begin{array}{c}\text { H.Chug } \\
\text { (unpublished) }\end{array}$ \\
\hline
\end{tabular}


Table 4.4-Continued from previous page

\begin{tabular}{|c|c|c|c|c|c|c|c|c|c|c|c|}
\hline \multirow[b]{2}{*}{ Plasmid } & \multirow[b]{2}{*}{ Construct } & \multirow[b]{2}{*}{ Marker } & \multirow[b]{2}{*}{ Ori } & \multicolumn{4}{|c|}{ Expression conditions } & \multicolumn{3}{|c|}{ Purification Conditions } & \multirow[b]{2}{*}{ Reference } \\
\hline & & & & Temp. & Duration & Strain & $\begin{array}{c}\text { IPTG } \\
(\mu \mathrm{M})\end{array}$ & buffer & Elution & $\begin{array}{l}\text { Gel- fil- } \\
\text { tration }\end{array}$ & \\
\hline CHC12 & $\begin{array}{l}\mathrm{H}_{14} \text {-brSUMO- } \\
\text { Crm1 K568R }\end{array}$ & Kan & ColE1 & $18^{\circ} \mathrm{C}$ & $\mathrm{o} / \mathrm{n}$ & $\begin{array}{c}\text { NEB } \\
\text { Express }\end{array}$ & 200 & $50 \mathrm{~T}, 200 \mathrm{~N}, 2 \mathrm{I}, 1 \mathrm{D}$ & Protease & - & $\begin{array}{c}\text { H.Chug } \\
\text { (unpublished) }\end{array}$ \\
\hline pSF1001 & $\begin{array}{l}\mathrm{H}_{14}-\mathrm{TEV}- \\
\text { mCherry }\end{array}$ & Kan & ColE1 & $30^{\circ} \mathrm{C}$ & $3 \mathrm{~h}$ & $\begin{array}{c}\text { NEB } \\
\text { Express }\end{array}$ & 200 & $50 \mathrm{~T}, 500 \mathrm{~N}$ & $500 \mathrm{I}$ & - & $\begin{array}{c}\text { S.Frey } \\
\text { (unpublished) }\end{array}$ \\
\hline pSF779 & $\begin{array}{c}\mathrm{H}_{14}-\mathrm{TEV}- \\
\left.\text { mCherry }_{\mathrm{cys}}\right)\end{array}$ & Kan & ColE1 & $30^{\circ} \mathrm{C}$ & $3 \mathrm{~h}$ & $\begin{array}{c}\text { NEB } \\
\text { Express }\end{array}$ & 200 & $50 \mathrm{~T}, 500 \mathrm{~N}$ & $500 \mathrm{I}$ & - & $\begin{array}{c}\text { S.Frey } \\
\text { (unpublished) }\end{array}$ \\
\hline pSF2036 & $\begin{array}{c}\mathrm{H}_{14} \text {-IF2d1- } \\
\text { brSUMO- } \\
\text { Streptavidin }_{(\mathrm{cys})}\end{array}$ & Kan & ColE1 & $30^{\circ} \mathrm{C}$ & $3 \mathrm{~h}$ & $\begin{array}{c}\text { NEB } \\
\text { Express }\end{array}$ & 200 & $50 \mathrm{~T}, 500 \mathrm{~N}$ & Protease & - & $\begin{array}{c}\text { S.Frey } \\
\text { (unpublished) }\end{array}$ \\
\hline pSF397 & $\begin{array}{c}\mathrm{H}_{14}-\mathrm{TEV}- \\
\text { ProteinA } \\
\text { (short) }\end{array}$ & Amp & ColE1 & $30^{\circ} \mathrm{C}$ & $3 \mathrm{~h}$ & $\begin{array}{c}\text { NEB } \\
\text { Express }\end{array}$ & 200 & $50 \mathrm{~T}, 500 \mathrm{~N}$ & $500 \mathrm{I}$ & - & $\begin{array}{c}\text { S.Frey } \\
\text { (unpublished) }\end{array}$ \\
\hline pDG2376 & $\mathrm{H}_{14}-\mathrm{ZpA} 963_{\text {(cys) }}$ & Kan & ColE1 & $30^{\circ} \mathrm{C}$ & $3 \mathrm{~h}$ & $\begin{array}{c}\text { NEB } \\
\text { Express }\end{array}$ & 200 & $50 \mathrm{~T}, 500 \mathrm{~N}$ & $1000 \mathrm{I}$ & + & $\begin{array}{c}\text { D.Görlich } \\
\text { (unpublished) }\end{array}$ \\
\hline pSF371 & $\begin{array}{l}\mathrm{H}_{10}-\mathrm{TEV}- \\
\operatorname{mRFP}_{(\mathrm{cys})}\end{array}$ & Amp & ColE1 & $30^{\circ} \mathrm{C}$ & $3 \mathrm{~h}$ & $\begin{array}{c}\text { NEB } \\
\text { Express }\end{array}$ & 200 & $50 \mathrm{~T}, 500 \mathrm{~N}$ & $500 \mathrm{I}$ & - & $\begin{array}{c}\text { S.Frey } \\
\text { (unpublished) }\end{array}$ \\
\hline pSF428 & $\begin{array}{c}\mathrm{H}_{14} \text {-Avi- } \\
\text { SUMOStar-Impß } \\
\text { (loop mutant) }\end{array}$ & Kan & ColE1 & $30^{\circ} \mathrm{C}$ & $3 \mathrm{~h}$ & $\begin{array}{c}\text { NEB } \\
\text { Express }\end{array}$ & 100 & $50 \mathrm{~T}, 500 \mathrm{~N}$ & $500 \mathrm{I}$ & - & This study \\
\hline pSF395 & $\begin{array}{c}\mathrm{H}_{14-\mathrm{ZZ}-} \\
\text { SUMOStar- } \\
\text { Spacer-(cys) }\end{array}$ & Kan & ColE1 & $30^{\circ} \mathrm{C}$ & $3 \mathrm{~h}$ & $\begin{array}{c}\text { NEB } \\
\text { Express }\end{array}$ & 100 & $50 \mathrm{~T}, 500 \mathrm{~N}$ & $500 \mathrm{I}$ & - & This study \\
\hline pMAtx38 & $\begin{array}{c}\mathrm{H}_{14} \text {-brSUMO- } \\
\text { Avi-Ataxin3(15Q) }\end{array}$ & Kan & ColE1 & & & & vided by & .Aksu & & & $\begin{array}{c}\text { M.Aksu } \\
\text { (unpublished) }\end{array}$ \\
\hline pТР036 & $\begin{array}{c}\mathrm{H}_{14}-\mathrm{MBP}- \\
\text { brSUMO- } \\
\text { Avi-Spacer- } x t \\
\text { Nup98 }_{(486-866)}\end{array}$ & Kan & CoLE1 & & & & ided by & Pleiner & & & $\begin{array}{c}\text { T.Pleiner } \\
\text { (unpublished) }\end{array}$ \\
\hline
\end{tabular}


Table 4.4-Continued from previous page

\begin{tabular}{|c|c|c|c|c|c|c|c|c|c|c|c|}
\hline \multirow[b]{2}{*}{ Plasmid } & \multirow[b]{2}{*}{ Construct } & \multirow[b]{2}{*}{ Marker } & \multirow[b]{2}{*}{ Ori } & \multicolumn{4}{|c|}{ Expression conditions } & \multicolumn{3}{|c|}{ Purification Conditions } & \multirow[b]{2}{*}{ Reference } \\
\hline & & & & Temp. & Duration & Strain & $\begin{array}{c}\text { IPTG } \\
(\mu \mathbf{M})\end{array}$ & buffer & Elution & $\begin{array}{l}\text { Gel- fil- } \\
\text { tration }\end{array}$ & \\
\hline JS429 & $\begin{array}{c}\mathrm{H}_{14} \text {-brSUMO- } \\
\text { Streptavidin }_{\text {(short) }} \\
\text { Spacer-(cys) }^{-} \\
\end{array}$ & Kan & ColE1 & $30^{\circ} \mathrm{C}$ & $3 \mathrm{~h}$ & $\begin{array}{c}\text { NEB } \\
\text { Express }\end{array}$ & 200 & $50 \mathrm{~T}, 500 \mathrm{~N}$ & Protease & - & This study \\
\hline JS439 & $\begin{array}{c}\mathrm{H}_{14} \text {-brSUMO- } \\
\text { Streptactin }_{\text {(short) }} \\
\text { Spacer- }^{-} \text {(cys) }\end{array}$ & Kan & ColE1 & $30^{\circ} \mathrm{C}$ & $3 \mathrm{~h}$ & $\begin{array}{c}\text { NEB } \\
\text { Express }\end{array}$ & 200 & $50 \mathrm{~T}, 500 \mathrm{~N}$ & Protease & - & This study \\
\hline pDG2443 & $\begin{array}{c}\mathrm{H}_{14^{-}} \\
\text {Barnase }_{(\mathrm{H} 102 \mathrm{D})}\end{array}$ & Kan & ColE1 & $30^{\circ} \mathrm{C}$ & $3 \mathrm{~h}$ & $\begin{array}{c}\text { NEB } \\
\text { Express }\end{array}$ & 200 & $50 \mathrm{~T}, 300 \mathrm{~N}, 2 \mathrm{I}, 1 \mathrm{D}$ & $300 \mathrm{I}$ & + & $\begin{array}{c}\text { D.Görlich } \\
\text { (unpublished) }\end{array}$ \\
\hline pDG2444 & $\begin{array}{c}\mathrm{H}_{14^{-}} \\
\text {Barnase }_{(\mathrm{H} 102 \mathrm{~F})}\end{array}$ & Kan & ColE1 & $30^{\circ} \mathrm{C}$ & $3 \mathrm{~h}$ & $\begin{array}{c}\text { NEB } \\
\text { Express }\end{array}$ & 200 & $50 \mathrm{~T}, 300 \mathrm{~N}, 2 \mathrm{I}, 1 \mathrm{D}$ & - & - & $\begin{array}{c}\text { D.Görlich } \\
\text { (unpublished) }\end{array}$ \\
\hline pDG2445 & $\begin{array}{c}\mathrm{H}_{14^{-}} \\
\text {Barnase }_{(\mathrm{H} 102 \mathrm{M})}\end{array}$ & Kan & ColE1 & $30^{\circ} \mathrm{C}$ & $3 \mathrm{~h}$ & $\begin{array}{c}\text { NEB } \\
\text { Express }\end{array}$ & 200 & $50 \mathrm{~T}, 300 \mathrm{~N}, 2 \mathrm{I}, 1 \mathrm{D}$ & - & - & $\begin{array}{c}\text { D.Görlich } \\
\text { (unpublished) }\end{array}$ \\
\hline pDG2446 & $\begin{array}{c}\mathrm{H}_{14^{-}} \\
\text {Barnase }_{(\mathrm{H} 102 \mathrm{~A})}\end{array}$ & Kan & ColE1 & $30^{\circ} \mathrm{C}$ & $3 \mathrm{~h}$ & $\begin{array}{c}\text { NEB } \\
\text { Express }\end{array}$ & 200 & $50 \mathrm{~T}, 300 \mathrm{~N}, 2 \mathrm{I}, 1 \mathrm{D}$ & - & - & $\begin{array}{c}\text { D.Görlich } \\
\text { (unpublished) }\end{array}$ \\
\hline pDG2451 & $\begin{array}{l}\text { ZZ-brNEDD8- } \\
\operatorname{Barstar}_{(\mathrm{C} 42 \mathrm{~K})}\end{array}$ & Kan & ColE1 & $30^{\circ} \mathrm{C}$ & $3 \mathrm{~h}$ & $\begin{array}{c}\text { NEB } \\
\text { Express }\end{array}$ & 200 & $50 \mathrm{~T}, 300 \mathrm{~N}, 2 \mathrm{I}, 1 \mathrm{D}$ & $300 \mathrm{I}$ & + & $\begin{array}{c}\text { D.Görlich } \\
\text { (unpublished) }\end{array}$ \\
\hline pDG2452 & $\begin{array}{l}\text { ZZ-brNEDD8- } \\
\operatorname{Barstar}_{(\mathrm{C} 42 \mathrm{~A})}\end{array}$ & Kan & ColE1 & $30^{\circ} \mathrm{C}$ & $3 \mathrm{~h}$ & $\begin{array}{c}\text { NEB } \\
\text { Express }\end{array}$ & 200 & $50 \mathrm{~T}, 300 \mathrm{~N}, 2 \mathrm{I}, 1 \mathrm{D}$ & - & - & $\begin{array}{c}\text { D.Görlich } \\
\text { (unpublished) }\end{array}$ \\
\hline pDG2453 & $\begin{array}{l}\text { ZZ-brNEDD8- } \\
\text { Barstar }_{(\mathrm{C} 42 \mathrm{~V})}\end{array}$ & Kan & ColE1 & $30^{\circ} \mathrm{C}$ & $3 \mathrm{~h}$ & $\begin{array}{c}\text { NEB } \\
\text { Express }\end{array}$ & 200 & $50 \mathrm{~T}, 300 \mathrm{~N}, 2 \mathrm{I}, 1 \mathrm{D}$ & - & - & $\begin{array}{c}\text { D.Görlich } \\
\text { (unpublished) }\end{array}$ \\
\hline pDG2454 & $\begin{array}{l}\text { ZZ-brNEDD8- } \\
\operatorname{Barstar}_{(\mathrm{C} 42 \mathrm{I})}\end{array}$ & Kan & ColE1 & $30^{\circ} \mathrm{C}$ & $3 \mathrm{~h}$ & $\begin{array}{c}\text { NEB } \\
\text { Express }\end{array}$ & 200 & $50 \mathrm{~T}, 300 \mathrm{~N}, 2 \mathrm{I}, 1 \mathrm{D}$ & - & - & $\begin{array}{c}\text { D.Görlich } \\
\text { (unpublished) }\end{array}$ \\
\hline pDG2455 & ZZ-Barstar $_{(\mathrm{C} 42 \mathrm{~K})}$ & Kan & ColE1 & $30^{\circ} \mathrm{C}$ & $3 \mathrm{~h}$ & $\begin{array}{c}\text { NEB } \\
\text { Express }\end{array}$ & 200 & $50 \mathrm{~T}, 300 \mathrm{~N}, 2 \mathrm{I}, 1 \mathrm{D}$ & - & - & $\begin{array}{c}\text { D.Görlich } \\
\text { (unpublished) }\end{array}$ \\
\hline pDG2456 & ZZ-Barstar $(\mathrm{C} 42 \mathrm{~A})$ & Kan & ColE1 & $30^{\circ} \mathrm{C}$ & $3 \mathrm{~h}$ & $\begin{array}{c}\text { NEB } \\
\text { Express }\end{array}$ & 200 & $50 \mathrm{~T}, 300 \mathrm{~N}, 2 \mathrm{I}, 1 \mathrm{D}$ & - & - & $\begin{array}{c}\text { D.Görlich } \\
\text { (unpublished) }\end{array}$ \\
\hline pDG2457 & ZZ-Barstar $(\mathrm{C} 42 \mathrm{~V})$ & Kan & ColE1 & $30^{\circ} \mathrm{C}$ & $3 \mathrm{~h}$ & $\begin{array}{c}\text { NEB } \\
\text { Express }\end{array}$ & 200 & $50 \mathrm{~T}, 300 \mathrm{~N}, 2 \mathrm{I}, 1 \mathrm{D}$ & - & - & $\begin{array}{c}\text { D.Görlich } \\
\text { (unpublished) }\end{array}$ \\
\hline
\end{tabular}


Table 4.4-Continued from previous page

\begin{tabular}{|c|c|c|c|c|c|c|c|c|c|c|c|}
\hline \multirow[b]{2}{*}{ Plasmid } & \multirow[b]{2}{*}{ Construct } & \multirow[b]{2}{*}{ Marker } & \multirow[b]{2}{*}{ Ori } & \multicolumn{4}{|c|}{ Expression conditions } & \multicolumn{3}{|c|}{ Purification Conditions } & \multirow[b]{2}{*}{ Reference } \\
\hline & & & & Temp. & Duration & Strain & $\begin{array}{c}\text { IPTG } \\
(\mu \mathbf{M})\end{array}$ & buffer & Elution & $\begin{array}{l}\text { Gel- fil- } \\
\text { tration }\end{array}$ & \\
\hline pDG2458 & $\mathrm{ZZ}_{-\operatorname{Barstar}_{(\mathrm{C} 42 \mathrm{I})}}$ & Kan & ColE1 & $30^{\circ} \mathrm{C}$ & $3 \mathrm{~h}$ & $\begin{array}{c}\text { NEB } \\
\text { Express }\end{array}$ & 200 & $50 \mathrm{~T}, 300 \mathrm{~N}, 2 \mathrm{I}, 1 \mathrm{D}$ & - & - & $\begin{array}{c}\text { D.Görlich } \\
\text { (unpublished) }\end{array}$ \\
\hline JS450 & $\begin{array}{c}\mathrm{H}_{14}-\mathrm{ZZ}- \\
\text { brSUMO-Barstar }\end{array}$ & Kan & ColE1 & $30^{\circ} \mathrm{C}$ & $3 \mathrm{~h}$ & $\begin{array}{c}\text { NEB } \\
\text { Express }\end{array}$ & 200 & $50 \mathrm{~T}, 300 \mathrm{~N}, 2 \mathrm{I}, 1 \mathrm{D}$ & - & - & This study \\
\hline JS453 & $\begin{array}{l}\mathrm{H}_{14} \text {-ZZ-brSUMO- } \\
\text { Barstar (GKKVT) }\end{array}$ & Kan & ColE1 & $30^{\circ} \mathrm{C}$ & $3 \mathrm{~h}$ & $\begin{array}{c}\text { NEB } \\
\text { Express }\end{array}$ & 200 & $50 \mathrm{~T}, 300 \mathrm{~N}, 2 \mathrm{I}, 1 \mathrm{D}$ & Protease & - & This study \\
\hline JS454 & $\begin{array}{c}\mathrm{H}_{14}-\mathrm{ZZ}- \\
\text { brSUMO-Barstar } \\
(\mathrm{GKKVT}, \mathrm{I} 87 \mathrm{E})\end{array}$ & Kan & ColE1 & $30^{\circ} \mathrm{C}$ & $3 \mathrm{~h}$ & $\begin{array}{c}\text { NEB } \\
\text { Express }\end{array}$ & 200 & $50 \mathrm{~T}, 300 \mathrm{~N}, 2 \mathrm{I}, 1 \mathrm{D}$ & Protease & - & This study \\
\hline JS455 & $\begin{array}{l}\mathrm{H}_{14} \text {-ZZ-brSUMO- } \\
\text { Barstar (GVKVT) }\end{array}$ & Kan & ColE1 & $30^{\circ} \mathrm{C}$ & $3 \mathrm{~h}$ & $\begin{array}{c}\text { NEB } \\
\text { Express }\end{array}$ & 200 & $-\mathrm{D}$ & - & - & This study \\
\hline JS456 & $\begin{array}{c}\mathrm{H}_{14}-\mathrm{ZZ}- \\
\text { brSUMO-Barstar } \\
(\mathrm{GVKVT}, \text { I87E }) \\
\end{array}$ & Kan & ColE1 & $30^{\circ} \mathrm{C}$ & $3 \mathrm{~h}$ & $\begin{array}{c}\text { NEB } \\
\text { Express }\end{array}$ & 200 & $50 \mathrm{~T}, 300 \mathrm{~N}, 2 \mathrm{I}, 1 \mathrm{D}$ & Protease & - & This study \\
\hline JS457 & $\begin{array}{c}\mathrm{H}_{14}-\mathrm{ZZ} \text {-brSUMO- } \\
\text { Barstar }_{(\mathrm{I} 87 \mathrm{E})}\end{array}$ & Kan & ColE1 & $30^{\circ} \mathrm{C}$ & $3 \mathrm{~h}$ & $\begin{array}{c}\text { NEB } \\
\text { Express }\end{array}$ & 200 & - & - & - & This study \\
\hline JS458 & $\begin{array}{c}\mathrm{H}_{14}-\mathrm{ZZ}- \\
\text { brSUMO-Barstar } \\
(\mathrm{GIKVT}, \mathrm{I} 87 \mathrm{E})\end{array}$ & Kan & ColE1 & $30^{\circ} \mathrm{C}$ & $3 \mathrm{~h}$ & $\begin{array}{c}\text { NEB } \\
\text { Express }\end{array}$ & 200 & $50 \mathrm{~T}, 300 \mathrm{~N}, 2 \mathrm{I}, 1 \mathrm{D}$ & Protease & - & This study \\
\hline JS459 & $\begin{array}{c}\mathrm{H}_{14}-\mathrm{ZZ}- \\
\text { brSUMO-Barstar } \\
\text { (GVKIT, I87E) } \\
\end{array}$ & Kan & ColE1 & $30^{\circ} \mathrm{C}$ & $3 \mathrm{~h}$ & $\begin{array}{c}\text { NEB } \\
\text { Express }\end{array}$ & 200 & $50 \mathrm{~T}, 300 \mathrm{~N}, 2 \mathrm{I}, 1 \mathrm{D}$ & Protease & - & This study \\
\hline JS460 & $\begin{array}{c}\mathrm{H}_{14-\mathrm{ZZ}-} \\
\text { brSUMO-Barstar } \\
(\mathrm{GIKIT}, \mathrm{I} 87 \mathrm{E}) \\
\end{array}$ & Kan & ColE1 & $30^{\circ} \mathrm{C}$ & $3 \mathrm{~h}$ & $\begin{array}{c}\text { NEB } \\
\text { Express }\end{array}$ & 200 & $50 \mathrm{~T}, 300 \mathrm{~N}, 2 \mathrm{I}, 1 \mathrm{D}$ & Protease & - & This study \\
\hline JS465 & $\begin{array}{c}\mathrm{H}_{14}-\mathrm{ZZ}- \\
\text { brSUMO-Barstar } \\
\text { (GVKVT, VEL, I87E) } \\
\end{array}$ & Kan & ColE1 & $30^{\circ} \mathrm{C}$ & $3 \mathrm{~h}$ & $\begin{array}{c}\text { NEB } \\
\text { Express }\end{array}$ & 200 & $50 \mathrm{~T}, 300 \mathrm{~N}, 2 \mathrm{I}, 1 \mathrm{D}$ & Protease & - & This study \\
\hline
\end{tabular}


Table 4.4-Continued from previous page

\begin{tabular}{|c|c|c|c|c|c|c|c|c|c|c|c|}
\hline \multirow[b]{2}{*}{ Plasmid } & \multirow[b]{2}{*}{ Construct } & \multirow[b]{2}{*}{ Marker } & \multirow[b]{2}{*}{ Ori } & \multicolumn{4}{|c|}{ Expression conditions } & \multicolumn{3}{|c|}{ Purification Conditions } & \multirow[b]{2}{*}{ Reference } \\
\hline & & & & Temp. & Duration & Strain & $\begin{array}{c}\text { IPTG } \\
(\mu \mathrm{M})\end{array}$ & buffer & Elution & $\begin{array}{l}\text { Gel- fil- } \\
\text { tration }\end{array}$ & \\
\hline JS466 & $\begin{array}{c}\mathrm{H}_{14}-\mathrm{ZZ}- \\
\text { brSUMO-Barstar } \\
\text { (GVKVT, IEL, I87E) }\end{array}$ & Kan & ColE1 & $30^{\circ} \mathrm{C}$ & $3 \mathrm{~h}$ & $\begin{array}{c}\text { NEB } \\
\text { Express }\end{array}$ & 200 & $50 \mathrm{~T}, 300 \mathrm{~N}, 2 \mathrm{I}, 1 \mathrm{D}$ & Protease & - & This study \\
\hline JS467 & $\begin{array}{c}\mathrm{H}_{14}-\mathrm{ZZ}- \\
\text { brSUMO-Barstar } \\
\text { (GVKVT, VEF, I87E) } \\
\end{array}$ & Kan & ColE1 & $30^{\circ} \mathrm{C}$ & $3 \mathrm{~h}$ & $\begin{array}{c}\text { NEB } \\
\text { Express }\end{array}$ & 200 & $50 \mathrm{~T}, 300 \mathrm{~N}, 2 \mathrm{I}, 1 \mathrm{D}$ & Protease & - & This study \\
\hline pTP103 & $\begin{array}{l}\text { pelB-CDN14- } \\
\text { HA-Amber-pIII }\end{array}$ & Amp & ColE1 & \multicolumn{7}{|c|}{ used for Phage Display } & $\begin{array}{c}\text { T.Pleiner } \\
\text { (unpublished) }\end{array}$ \\
\hline JS468 & $\begin{array}{c}\text { pelB-JS456- } \\
\text { HA-Amber-pIII }\end{array}$ & Amp & ColE1 & \multicolumn{7}{|c|}{ used for Phage Display } & This study \\
\hline JS494 & $\begin{array}{c}\mathrm{H}_{14} \text {-ZZ-brSUMO- } \\
\text { G.t.Barstar }\end{array}$ & Kan & ColE1 & $30^{\circ} \mathrm{C}$ & $3 \mathrm{~h}$ & $\begin{array}{c}\text { NEB } \\
\text { Express }\end{array}$ & 200 & $50 \mathrm{~T}, 300 \mathrm{~N}, 2 \mathrm{I}, 1 \mathrm{D}$ & - & - & This study \\
\hline JS495 & $\begin{array}{c}\mathrm{H}_{14} \text {-ZZ-brSUMO- } \\
\text { B.puBarstar }\end{array}$ & Kan & ColE1 & $30^{\circ} \mathrm{C}$ & $3 \mathrm{~h}$ & $\begin{array}{c}\text { NEB } \\
\text { Express }\end{array}$ & 200 & $50 \mathrm{~T}, 300 \mathrm{~N}, 2 \mathrm{I}, 1 \mathrm{D}$ & - & - & This study \\
\hline JS496 & $\begin{array}{c}\mathrm{H}_{14} \text {-ZZ-brSUMO- } \\
\text { B.pl1 Barstar }\end{array}$ & Kan & ColE1 & $30^{\circ} \mathrm{C}$ & $3 \mathrm{~h}$ & $\begin{array}{c}\text { NEB } \\
\text { Express }\end{array}$ & 200 & $50 \mathrm{~T}, 300 \mathrm{~N}, 2 \mathrm{I}, 1 \mathrm{D}$ & - & - & This study \\
\hline JS497 & $\begin{array}{c}\mathrm{H}_{14}-\mathrm{ZZ} \text {-brSUMO- } \\
\text { B.pl2Barstar }\end{array}$ & Kan & ColE1 & $30^{\circ} \mathrm{C}$ & $3 \mathrm{~h}$ & $\begin{array}{c}\text { NEB } \\
\text { Express }\end{array}$ & 200 & $50 \mathrm{~T}, 300 \mathrm{~N}, 2 \mathrm{I}, 1 \mathrm{D}$ & - & - & This study \\
\hline JS489 & $\begin{array}{c}\mathrm{H}_{14}-\mathrm{ZZ} \text {-brSUMO- } \\
\text { G.t.Barnase }\end{array}$ & Kan & ColE1 & $30^{\circ} \mathrm{C}$ & $3 \mathrm{~h}$ & $\begin{array}{c}\text { NEB } \\
\text { Express }\end{array}$ & 200 & $50 \mathrm{~T}, 300 \mathrm{~N}, 2 \mathrm{I}, 1 \mathrm{D}$ & - & - & This study \\
\hline JS498 & $\begin{array}{c}\mathrm{H}_{14} \text {-brSUMO- } \\
\text { G.t.Barnase } \\
(42-154) \\
\end{array}$ & Kan & ColE1 & $30^{\circ} \mathrm{C}$ & $3 \mathrm{~h}$ & $\begin{array}{c}\text { NEB } \\
\text { Express }\end{array}$ & 200 & $50 \mathrm{~T}, 300 \mathrm{~N}, 2 \mathrm{I}, 1 \mathrm{D}$ & - & - & This study \\
\hline JS500 & $\begin{array}{c}\mathrm{H}_{14} \text {-brSUMO- } \\
\text { G.t.Barnase } \\
(36-154)\end{array}$ & Kan & ColE1 & $30^{\circ} \mathrm{C}$ & $3 \mathrm{~h}$ & $\begin{array}{c}\text { NEB } \\
\text { Express }\end{array}$ & 200 & $50 \mathrm{~T}, 300 \mathrm{~N}, 2 \mathrm{I}, 1 \mathrm{D}$ & - & - & This study \\
\hline
\end{tabular}


Table 4.4-Continued from previous page

\begin{tabular}{|c|c|c|c|c|c|c|c|c|c|c|c|}
\hline \multirow[b]{2}{*}{ Plasmid } & \multirow[b]{2}{*}{ Construct } & \multirow[b]{2}{*}{ Marker } & \multirow[b]{2}{*}{ Ori } & \multicolumn{4}{|c|}{ Expression conditions } & \multicolumn{3}{|c|}{ Purification Conditions } & \multirow[b]{2}{*}{ Reference } \\
\hline & & & & Temp. & Duration & Strain & $\begin{array}{c}\text { IPTG } \\
(\mu \mathbf{M})\end{array}$ & buffer & Elution & $\begin{array}{l}\text { Gel- fil- } \\
\text { tration }\end{array}$ & \\
\hline JS501 & $\begin{array}{c}\mathrm{H}_{14} \text {-brSUMO- } \\
\text { G.t.Barnase } \\
(32-154) \\
\end{array}$ & Kan & ColE1 & $30^{\circ} \mathrm{C}$ & $3 \mathrm{~h}$ & $\begin{array}{c}\text { NEB } \\
\text { Express }\end{array}$ & 200 & $50 \mathrm{~T}, 300 \mathrm{~N}, 2 \mathrm{I}, 1 \mathrm{D}$ & - & - & This study \\
\hline JS502 & $\begin{array}{c}\mathrm{H}_{14} \text {-brSUMO- } \\
\text { G.t.Barnase } \\
(19-154)\end{array}$ & Kan & ColE1 & $30^{\circ} \mathrm{C}$ & $3 \mathrm{~h}$ & $\begin{array}{c}\text { NEB } \\
\text { Express }\end{array}$ & 200 & $50 \mathrm{~T}, 300 \mathrm{~N}, 2 \mathrm{I}, 1 \mathrm{D}$ & - & - & This study \\
\hline JS503 & $\begin{array}{c}\text { ZZ-brNEDD8- } \\
\text { G.t.Barstar }\end{array}$ & Kan & ColE1 & $30^{\circ} \mathrm{C}$ & $3 \mathrm{~h}$ & $\begin{array}{c}\text { NEB } \\
\text { Express }\end{array}$ & 200 & $50 \mathrm{~T}, 300 \mathrm{~N}, 2 \mathrm{I}, 1 \mathrm{D}$ & Protease & + & This study \\
\hline JS504 & $\begin{array}{c}\text { ZZ-brNEDD8- } \\
\text { B.puBarstar }\end{array}$ & Kan & ColE1 & $30^{\circ} \mathrm{C}$ & $3 \mathrm{~h}$ & $\begin{array}{c}\text { NEB } \\
\text { Express }\end{array}$ & 200 & $50 \mathrm{~T}, 300 \mathrm{~N}, 2 \mathrm{I}, 1 \mathrm{D}$ & Protease & + & This study \\
\hline JS505 & $\begin{array}{l}\text { ZZ-brNEDD8- } \\
\text { B.pl1 Barstar }\end{array}$ & Kan & ColE1 & $30^{\circ} \mathrm{C}$ & $3 \mathrm{~h}$ & $\begin{array}{c}\text { NEB } \\
\text { Express }\end{array}$ & 200 & $50 \mathrm{~T}, 300 \mathrm{~N}, 2 \mathrm{I}, 1 \mathrm{D}$ & Protease & + & This study \\
\hline JS506 & $\begin{array}{l}\text { ZZ-brNEDD8- } \\
\text { B.pl2Barstar }\end{array}$ & Kan & ColE1 & $30^{\circ} \mathrm{C}$ & $3 \mathrm{~h}$ & $\begin{array}{c}\text { NEB } \\
\text { Express }\end{array}$ & 200 & $50 \mathrm{~T}, 300 \mathrm{~N}, 2 \mathrm{I}, 1 \mathrm{D}$ & Protease & + & This study \\
\hline JS514 & ZZ-G.t.Barstar & Kan & ColE1 & $30^{\circ} \mathrm{C}$ & $3 \mathrm{~h}$ & $\begin{array}{c}\text { NEB } \\
\text { Express }\end{array}$ & 200 & $50 \mathrm{~T}, 300 \mathrm{~N}, 2 \mathrm{I}, 1 \mathrm{D}$ & - & - & This study \\
\hline JS515 & ZZ-B.puBarstar & Kan & ColE1 & $30^{\circ} \mathrm{C}$ & $3 \mathrm{~h}$ & $\begin{array}{c}\text { NEB } \\
\text { Express }\end{array}$ & 200 & $50 \mathrm{~T}, 300 \mathrm{~N}, 2 \mathrm{I}, 1 \mathrm{D}$ & - & - & This study \\
\hline JS516 & ZZ-B.pl1 Barstar & Kan & ColE1 & $30^{\circ} \mathrm{C}$ & $3 \mathrm{~h}$ & $\begin{array}{c}\text { NEB } \\
\text { Express }\end{array}$ & 200 & $50 \mathrm{~T}, 300 \mathrm{~N}, 2 \mathrm{I}, 1 \mathrm{D}$ & - & - & This study \\
\hline JS517 & ZZ-B.pl2Barstar & Kan & ColE1 & $30^{\circ} \mathrm{C}$ & $3 \mathrm{~h}$ & $\begin{array}{c}\text { NEB } \\
\text { Express }\end{array}$ & 200 & $50 \mathrm{~T}, 300 \mathrm{~N}, 2 \mathrm{I}, 1 \mathrm{D}$ & - & - & This study \\
\hline JS444 & $\begin{array}{c}\text { M- } \\
\text { Barnase }_{(\mathrm{H} 102 \mathrm{D})^{-}} \\
\text {brSUMO-GFP4 }^{-}\end{array}$ & Kan & ColE1 & $30^{\circ} \mathrm{C}$ & $3 \mathrm{~h}$ & $\begin{array}{c}\text { NEB } \\
\text { Express }\end{array}$ & 200 & $50 \mathrm{~T}, 300 \mathrm{~N}, 2 \mathrm{I}, 1 \mathrm{D}$ & $500 \mathrm{I}$ & - & This study \\
\hline JS527 & $\begin{array}{c}\mathrm{H}_{14} \text {-brSUMO- } \\
\text { G.t.Barnase } \\
(42-154) \\
\end{array}$ & Amp & pSC101 & $30^{\circ} \mathrm{C}$ & $3 \mathrm{~h}$ & $\begin{array}{c}\text { NEB } \\
\text { Express }\end{array}$ & 200 & $50 \mathrm{~T}, 300 \mathrm{~N}, 2 \mathrm{I}, 1 \mathrm{D}$ & Protease & + & This study \\
\hline
\end{tabular}


Table 4.4 - Continued from previous page

\begin{tabular}{|c|c|c|c|c|c|c|c|c|c|c|c|}
\hline \multirow[b]{2}{*}{ Plasmid } & \multirow[b]{2}{*}{ Construct } & \multirow[b]{2}{*}{ Marker } & \multirow[b]{2}{*}{ Ori } & \multicolumn{4}{|c|}{ Expression conditions } & \multicolumn{3}{|c|}{ Purification Conditions } & \multirow[b]{2}{*}{ Reference } \\
\hline & & & & Temp. & Duration & Strain & $\begin{array}{c}\text { IPTG } \\
(\mu \mathbf{M})\end{array}$ & buffer & Elution & $\begin{array}{l}\text { Gel- fil- } \\
\text { tration }\end{array}$ & \\
\hline JS528 & $\begin{array}{c}\mathrm{H}_{14} \text {-brSUMO- } \\
\text { G.t.Barnase } \\
(36-154)\end{array}$ & Amp & pSC101 & $30^{\circ} \mathrm{C}$ & $3 \mathrm{~h}$ & $\begin{array}{c}\text { NEB } \\
\text { Express }\end{array}$ & 200 & $50 \mathrm{~T}, 300 \mathrm{~N}, 2 \mathrm{I}, 1 \mathrm{D}$ & Protease & + & This study \\
\hline JS529 & $\begin{array}{c}\mathrm{H}_{14} \text {-brSUMO- } \\
\text { G.t.Barnase } \\
(32-154)\end{array}$ & Amp & pSC101 & $30^{\circ} \mathrm{C}$ & $3 \mathrm{~h}$ & $\begin{array}{c}\text { NEB } \\
\text { Express }\end{array}$ & 200 & $50 \mathrm{~T}, 300 \mathrm{~N}, 2 \mathrm{I}, 1 \mathrm{D}$ & - & Protease & This study \\
\hline pSF1389 & $\mathrm{H}_{14}$-brSENP1 & Amp & ColE1 & \multicolumn{7}{|c|}{ provided by S. Frey } & $\begin{array}{c}\text { Frey and } \\
\text { Görlich } \\
(2014 \mathrm{a})\end{array}$ \\
\hline pSF1443 & $\begin{array}{l}\mathrm{H}_{14} \text {-TEV- } \\
\text { brNEDP1 }\end{array}$ & Kan & ColE1 & \multicolumn{7}{|c|}{ provided by S. Frey } & $\begin{array}{c}\text { Frey and } \\
\text { Görlich } \\
(2014 \mathrm{a})\end{array}$ \\
\hline pSF1878 & ZZ-B.pl2Barstar & Kan & ColE1 & $180^{\circ} \mathrm{C}$ & $\mathrm{o} / \mathrm{nh}$ & $\begin{array}{c}\text { NEB } \\
\text { Express }\end{array}$ & 200 & $50 \mathrm{~T}, 300 \mathrm{~N}, 2 \mathrm{I}, 1 \mathrm{D}$ & $500 \mathrm{I}$ & + & $\begin{array}{c}\text { S.Frey } \\
\text { (unpublished) }\end{array}$ \\
\hline pSF965 & BirA & Spec & $\mathrm{p} 15 \mathrm{~A}$ & \multicolumn{7}{|c|}{ provided by S. Frey } & $\begin{array}{c}\text { S.Frey } \\
\text { (unpublished) }\end{array}$ \\
\hline
\end{tabular}




\subsection{Methods}

\subsubsection{Standard Techniques in Molecular Biology}

All standard methods in Molecular Biology were performed on the basis of Sambrook and Russell (2001).

\subsubsection{Polymerase Chain Reaction (PCR)}

Polymerase Chain reaction (PCR; Mullis et al., 1986) was used to amplify defined fragments from a DNA template. Synthesized primers (Sigma-Aldrich, Germany) allowed to introduce either endonuclease restriction sites on both ends of the generated DNA fragment or overlapping regions on both sites for cloning using Gibson Assembly (see 4.2.1.7).

A $50 \mu \mathrm{l}$ PCR reaction was composed as described below:



For general cloning, a PfuS polymerase Tripple Mix (100ng/ $\mu$ l PfuS DNA polymerase, $15 \mathrm{ng} / \mu \mathrm{l}$ Pab pyrophosphates, $2.5 \mathrm{ng} / \mu \mathrm{l}$ Pab dUTPase (all produced in the lab by S. Frey) and the corresponding 10x PfuS buffer (200 mM Tris/HCl pH9, $250 \mathrm{mM} \mathrm{KCl,} 15 \mathrm{mM}$ $\mathrm{MgSO}_{4}, 10 \mathrm{mM}\left(\mathrm{NH}_{4}\right)_{2} \mathrm{SO}_{4}, 1 \%$ Tween-20, $\left.1 \mathrm{mg} / \mathrm{ml} \mathrm{BSA}\right)$ was used.

PCR reactions were performed using a SensoQuest Lab Cycler, programmed with the required temperatures and duration times.

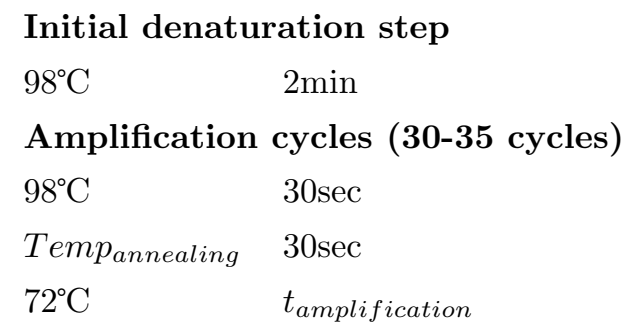

Completion step

$72^{\circ} \mathrm{C} \quad 5 \mathrm{~min}$

$10^{\circ} \mathrm{C} \quad \infty$

Tempannealing describes an average annealing temperature of a specific primer pair calculated by the Oligo software, $t_{\text {amplification }}$ describes the duration of amplification, depending 
on the size of the DNA fragment to be amplified and the amplification rate of the DNA polymerase ( $\sim 30 \mathrm{sec} / \mathrm{kb}$ for PfuS polymerase).

\subsubsection{Mutagenesis Polymerase Chain Reaction}

Mutagenesis PCR was performed to render DNA sequence in various ways: small parts of a gene can be deleted, single amino acids or short amino acid stretches can be exchanged or a single amino acid or a short amino acid stretch can be additionally added to the DNA sequence.

For this purpose PCR primers were designed that amplify the entire plasmid of interest therewhile introducing the intended changes by non-complementary base-pairing of the primers. For mutagenesis PCR the primers were ordered HPLC-purified and phosphorylated at the 5' end. The PCR reaction was performed as described in 4.2.1.1.

After the reaction, the PCR products were purified using the MSB Spin PCRapace Kit (STRATEC Biomedical AG, Germany) and further digested with $1 \mu$ l Dpn1 (the reaction contained NEB buffer 3 in a ratio of $1: 10$ ) over night at $37^{\circ} \mathrm{C}$ to remove the methylated template DNA. Subsequently, the reaction was purified by DNA gel purification (4.2.1.5) and ligated over night at $16^{\circ} \mathrm{C} .1 \mu \mathrm{l}$ ligation reaction was then transformed into E.coli cells and plated on selective medium.

\subsubsection{Error-prone Polymerase Chain Reaction}

Error-prone PCR reactions were performed to randomize a defined DNA sequence. Primers were synthesized flanking and amplifying the specific sequence during the reaction. Introduced endonuclease restriction sites on both ends of the DNA fragment enables subsequent cloning, e.g. in display vectors.

Error-prone PCR was performed using the JBS Error-Prone Kit (Jena Biosciences, Germany) according to the manufacturer's instruction.

\subsubsection{DNA Gel Electrophoresis}

Agarose gel electrophoresis was used to separate DNA fragments resulting from either PCR amplification or enzymatic restriction digest according to their size. Agarose concentrations varied from $0.8 \%(\mathrm{w} / \mathrm{v})$ to $2.0 \%(\mathrm{w} / \mathrm{v})$, depending on the expected fragment size. DNA samples were mixed in a 1:10 (v/v) ratio with Orange-G loading buffer. For reference, a standard marker (1kb DNA ladder, Fermentas, St.Leon-Roth, Germany) was included.

Electrophoresis was carried out at $\sim 160 \mathrm{~V}$ for 40 minutes. To visualize the DNA under UV light, $3 \mu \mathrm{l}$ of a $20 \mathrm{mg} / \mathrm{ml}$ ethidium bromide solution were added to $100 \mathrm{ml}$ agarose gel. Agarose gels were formed and run in 1x TAE buffer. 


\subsubsection{Purification of DNA Fragments from Agarose Gel and Concentration Determination}

After DNA gel electrophoresis, DNA fragments were cut from the gel and purified by using the Zymoclean Gel DNA recovery kit (Zymoclean Research, CA, USA) according to the manufacturer's instruction. In case of PCR products, the reactions were directly purified using the MSB Spin PCRapace Kit (STRATEC Biomedical AG, Germany).

DNA concentrations were determined with a NanoDrop-2000 spectrophotometer (Thermo Scientific, DE, USA).

\subsubsection{Enzymatic Restriction Digest}

Enzymatic restriction digest were performed to generate DNA fragments (vector backbones or insert) with complementary overhangs for further cloning.

All restriction endonucleases were obtained from NEB (New England Biolabs, Beverly, MA, USA). The cleavage reaction was performed according to the manufacturer's instructions using the provided buffers and reagents. The restriction enzymes were used in at least 2 -fold excess for $1 \mathrm{~h}$ reaction at $37^{\circ} \mathrm{C}$. A decreased amount of enzyme was added to the reaction when incubating the reaction over night.

In case of digested vector backbones, $1 \mu \mathrm{l}$ fast alkaline phosphatase (Fermentas, St.LeonRoth, Germany) was added subsequently and the reaction was further incubated at $37^{\circ} \mathrm{C}$ for 30 minutes. Dephosphorylation of the vector backbone prevents religation during following cloning steps.

\subsubsection{Gibson Assembly Cloning}

Gibson Assembly is an alternative, restriction enzyme free cloning method in which multiple double stranded DNA fragments are joined in a single isothermal reaction. Joining occurs via overlapping ends that should ideally have a melting temperature of $\geq 48^{\circ} \mathrm{C}$ (Gibson et al., 2009).

Primers containing the overlapping ends were designed using the Seqbuilder software and primers were synthesized by Sigma-Aldrich. Next, the individual DNA fragments were amplified by PCR and the reaction was digested with Dpn1 to remove all residual plasmid DNA. After purification via MSB Spin PCRapace Kit, the Gibson Assembly reaction was mixed as describes below: 


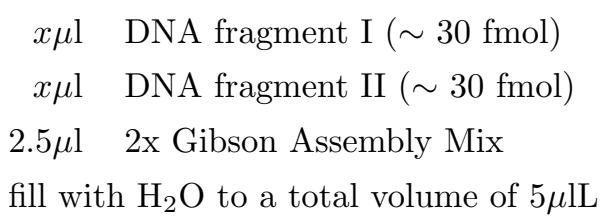

The reaction was incubated at $50^{\circ} \mathrm{C}$ for 30 minutes prior to transformation. After recovery the cells were plated on selective agar plates.

\subsubsection{Ligation of DNA}

Ligation was performed using the T4 ligase (prepared in the lab, 100ng/ $\mu \mathrm{l}$ ). Approximately $30 \mathrm{fmol}(20 \mathrm{ng} / \mathrm{kb})$ vector DNA and $60 \mathrm{fmol}(40 \mathrm{ng} / \mathrm{kb})$ insert DNA were mixed with $1 \mu \mathrm{l}$ T4 DNA ligase and $1 \mu \mathrm{l} 10 \mathrm{x}$ ligase buffer $\left(0.5 \mathrm{M}\right.$ Tris- $\mathrm{HCl} \mathrm{pH} 7.5,100 \mathrm{mM} \mathrm{MgCl}_{2}$, $100 \mathrm{mM}$ DTT, $10 \mathrm{mM}$ ATP, $250 \mu \mathrm{g} / \mathrm{ml} \mathrm{BSA}$ ) and filled up to a total volume of $10 \mu \mathrm{l}$ with $\mathrm{ddH}_{2} \mathrm{O}$.

After mixing, the ligation reaction was either incubated at $37^{\circ} \mathrm{C}$ for 30 minutes or overnight at $16^{\circ} \mathrm{C} .1 \mu \mathrm{l}$ ligation reaction was then used to transform $E$. coli cells by electroporation.

\subsubsection{Transformation of $E$. coli with DNA by electroporation}

$40 \mu \mathrm{l}$ electro-competent E.coli cell suspension (prepared in the department by G. Kopp) were mixed with either $1 \mu \mathrm{l}$ ligation reaction or 50ng plasmid DNA in electroporation cuvettes (Biorad, Burlington, USA) on ice. Electroporation was performed using a GenePulser (Biorad, Burlington, USA) according to manufacturers instructions. For recovery, cells were mixed with $1 \mathrm{ml}$ fresh $2 \mathrm{YT}$ media and incubated for $1 \mathrm{~h}$ at $37^{\circ} \mathrm{C}$ using a Thermomixer comfort (Eppendorf, Germany) shaking at $1300 \mathrm{rpm}$. Subsequently, cells were plated on selective agar plates and incubated over night at $37^{\circ} \mathrm{C}$. Alternatively, transformed cells were directly added to selective liquid culture and were incubated at $27^{\circ} \mathrm{C}$ or $37^{\circ} \mathrm{C}$.

\subsubsection{Validation of positive clones by testexpression}

Colonies on selective agar plates need to be analyzed for correctness prior to plasmid DNA preparation. Therefore, single colonies were carefully picked and resuspended in $200 \mu \mathrm{l} 2 \mathrm{YT}$ media containing the corresponding antibiotics. These small cultures were incubated at $37^{\circ} \mathrm{C}$ while shaking until they turn slightly turbid. $50 \mu \mathrm{l}$ were transferred to separate tubes for later inoculation of DNA plasmid preparation cultures. The remaining cultures were induced with $1 \mathrm{mM}$ IPTG (Isopropyl- $\beta$-D-thiogalactopyranosid) and incubated at $37^{\circ} \mathrm{C}$ for 2-3h. The cultures were spun down using a table-top centrifuge (Eppendorf, Germany) at 1300rpm. After discarding the supernatant, the cell pellet was resuspended in $80 \mu \mathrm{l}$ SDS loading buffer, heated to $95^{\circ} \mathrm{C}$ for 5 minutes and subsequently loaded on SDS-PAGE. 
The major expressed band should correspond to the expected size of the cloned construct. Cultures for DNA plasmid preparation were inoculated.

\subsubsection{Plasmid DNA Purification from E. coli}

Plasmid DNA from E. coli was either purified at analytical scale (mini preps) by using the NucleoSpin Plasmid Kit (Macherey Nagel, Germany) or at preparative scale (midi preps) using the NucleoBond PC100 Kit (Macherey Nagel, Germany), in each case according to the manufacturers instructions. Purification was mainly performed by G.Kopp.

$4 \mathrm{ml} \mathrm{LB}$ media containing the corresponding antibiotics were inoculated for analytical purification, $250 \mathrm{ml}$ cultures were inoculated for preparative purifications. The cultures were incubated over night at $37^{\circ} \mathrm{C}$.

\subsubsection{Primer Synthesis and DNA Sequencing}

Primers for sequencing purposes were designed using the SeqBuilder software (part of the DNAStar software package). Primers were mainly synthesized by Sigma-Aldrich, Germany, partially also by IBA GmBH, Germany.

DNA sequencing reactions of plasmids or purified DNA fragments were performed by SEQLAB Sequence Laboratories Göttingen GmbH, Germany. Results were analyzed using the SeqMan software (DNAStar).

\subsubsection{Protein Expression and Purification}

\subsubsection{Protein Expression}

All proteins used in this study were expressed in E. coli expression strains (see 4.1). Protein quality and yield greatly depend on a variety of different parameters such as used E. coli expression strains, expression conditions (expression time, expression temperature and induction strength) but also the expression construct itself (AT-content in the coding sequence, secondary structure of the resulting mRNA transcript, solubility enhancing fusion tags, etc.). Thus, the ideal construct design and expression conditions were determined for every protein produced and summarized in table 4.4 .

In general, the plasmid coding for the protein sequence of interest and an antibiotic resistance cassette was transformed into electro-competent $E$. coli cells, recovered in $1 \mathrm{ml} 2 \mathrm{YT}$ media for $1 \mathrm{~h}$ at $37^{\circ} \mathrm{C}$ and subsequently streaked out on agarose plates containing the corresponding antibiotics. After over night incubation at $37^{\circ} \mathrm{C}$, a single colony was inoculated in $100 \mathrm{ml}$ media (2YT or TB, depending on the protein expressed) supplemented with antibiotics and grown over night at $27^{\circ} \mathrm{C}-30^{\circ} \mathrm{C}$ while shaking at $95 \mathrm{rpm}$. Next, the cultures were diluted with $700 \mathrm{ml}$ fresh, antibiotic containing media and induced with IPTG (100- 
$200 \mu \mathrm{M})$. Ideally, the $\mathrm{OD}_{600}$ at the time point of induction should be between 0.8-1.0. The cultures were further incubated at the preferred expression temperature for an defined expression time while shaking with 85rpm.

Prior to harvest, the $\mathrm{OD}_{600}$ was measured and the culture was supplemented with $1 \mathrm{mM}$ PMSF (phenylmethanesulfonylfluoride) and 10mM EDTA in order to inhibit proteases during the following treatment. Bacterial cells were then pelleted by centrifugation $\left(4^{\circ} \mathrm{C}\right.$, 10 minutes, 4000rpm). The resulting bacterial pellet was resuspended in native resuspension buffer (if not stated otherwise: $50 \mathrm{mM}$ Tris/HCl, $\mathrm{pH} 7.5,300-500 \mathrm{mM} \mathrm{NaCl}, 2 \mathrm{mM}$ imidazole, $1 \mathrm{mM}$ DTT) to a final ratio of $3.5 \mathrm{OD}_{600}=45 \mathrm{ml}$. The cell suspension was then snap-frozen in liquid nitrogen and stored at $-80^{\circ} \mathrm{C}$ until further proceeding.

\subsubsection{Cell lysis}

Bacterial cell walls were disrupted by mechanical stress induced by a freeze/thawing cycle. In addition, the cells were further lysed by ultra-sonication, disrupting cell structures and shearing the released DNA to smaller fragments. Therefor resuspended cells were thawn in hand warm water and placed on ice after completeness. Sonication was performed using the Sonifier 450 (Branson, UK) for 2x 2minutes on ice with a maximum output and $40 \%$ duty cycle. To clear the supernatant from cell debris and insoluble proteins, the ruptured cells were ultracentrifuged for $1-2 \mathrm{~h}$ at $370.000 \mathrm{rpm}, 4^{\circ} \mathrm{C}$, using the T647.5 rotor (Sorvall).

\subsubsection{Native Protein Purification}

To separate the recombinantly expressed protein of interest from other proteins present in the cleared lysate, $\mathrm{Ni}^{2+}$ affinity chromatography was performed. The protein of interest was expressed as a fusion construct with a stretch of histidine residues $\left(\mathrm{H}_{14^{-}}\right.$- or $\mathrm{H}_{10^{-}}$tags). The imidazole ring of the histidine side chains can complex bivalent metal ions such as $\mathrm{Ni}^{2+}$ or $\mathrm{Co}^{2+}$ at physiological pH. By using a $6 \% \mathrm{Ni}^{2+}$-chelate $500 \AA$ silica matrix (prepared in the lab by D. Görlich) as affinity resin, tagged proteins of interest can be pulled out specifically whereas other proteins in the lysate show no or only little affinity towards the matrix and thus do not bind efficiantly.

Depending on the amount of expressed protein of interest in the lysate, an appropriate volume of $6 \% \mathrm{Ni}^{2+}$-chelate $500 \AA$ silica was equilibrated with the resuspension buffer, subsequently mixed with the cleared lysate and incubated for at least $1 \mathrm{~h}$ at $4^{\circ} \mathrm{C}$ while gently rotating to facilitate binding of the tagged protein to the affinity resin. The buffer contains low amounts of imidazole $(2 \mathrm{mM})$ to reduce unspecific binding of bacterial proteins. After incubation, the suspension was filled to a gravity flow column $(1.5 \times 1.0 \mathrm{~cm}$, Sigma-Aldrich) and the supernatant was collected and checked for remaining amount of the protein of interest subsequently. Ideally, the matrix should be slightly overloaded to avoid non- 
specific binding of bacteria proteins.

The matrix was then thoroughly washed with resuspension buffer. In special cases, either a high salt wash step (1M salt to remove RNA contaminations) or an ATP wash step (1-5mM ATP) to remove bound chaperones was included prior to elution. Elution was performed either by protease cleavage (see 4.2.2.4) or by addition of high concentrations of imidazole in the elution buffer. Depending on the protein of interest and the length of the used histidine stretch, concentrations between $300 \mathrm{mM}$ and $1 \mathrm{M}$ imidazole were used. Imidazole competes out the His-tagged proteins thereby displacing the protein of interest from the resin. Elution was performed in $500 \mu \mathrm{l}$ to $1 \mathrm{ml}$ steps, the fractions were tested for their protein content by quick staining with amid black solution $(1 \mu$ l of the elution fractions was spotted on nitrocellulose membrane and incubated for $1 \mathrm{~min}$ with the amido black staining solution) and the protein containing fractions were pooled.

The pooled protein elution was supplements with $250 \mathrm{mM}$ sucrose to prevent freezing damage and the protein concentrations were determined spectroscopically by absorption measurements at $280 \mathrm{~nm}$. Concentrations were then calculated using the following formula: $c[M]=A_{280}\left[\mathrm{~cm}^{-1}\right] / \epsilon_{280}\left[\mathrm{M}^{-1} \mathrm{~cm}^{-1}\right]$. Subsequently, the protein elution was snap-frozen in liquid nitrogen and stored at $-80^{\circ} \mathrm{C}$.

\subsubsection{Protease Cleavage}

Protein tags can be used for a variety of reasons, e.g. affinity tags such as the previously described poly-histidine tag to provide affinity towards the $\mathrm{Ni}^{2+}$-chelate $500 \AA$ silica. But tags can also function as expression or solubility enhancers for the protein of interest, such as the maltose binding protein (MBP). However, these tags can be rather bulky and might be disadvantageous for downstream applications. To efficiently remove these tags, specific endoprotease cleavage sites can be included in the expression construct. For this purpose, either the TEV protease recognition sequence, the SUMO protein or the NEDD8 protein (both from from Brachypodium distachyon) were fused between the N-terminal expression tag and the protein sequence of interest.

TEV protease is derived from the Tobacco etch virus. The virus encodes its genome as a single polyprotein which then has to be cleaved by specific proteases, one of them being the TEV protease. Cleavage was performed by direct addition of the TEV protease (produced in the lab by $\mathrm{S}$. Frey) to the $\mathrm{Ni}^{2+}$ elute. The cleavage reaction was performed for $1 \mathrm{~h}$ at $\mathrm{RT}$ or over night at $4^{\circ} \mathrm{C}$ using $\sim 10 \mu \mathrm{M}$ TEV protease for efficient cleavage. After cleavage, the solution was either applied to size exclusion chromatography to separate the protein of interest from the tag (see 4.2.2.8) or a reverse $\mathrm{Ni}^{2+}$ chromatography was performed. Here, the cleaved sample was applied once again to $6 \% \mathrm{Ni}^{2+}$-chelate $500 \AA$ silica. The cleaved off protein should have lost its ability to bind the resin whereas the tag should remain 
bound. The flow through after $1 \mathrm{~h}$ of incubation was collected in $0.5-1 \mathrm{ml}$ fractions and analyzed for their protein content using amido black staining. Protein containing fractions were pooled, the protein concentration was measured and the samples were snap-frozen in liquid nitrogen and stored at $-80^{\circ} \mathrm{C}$.

SUMO and NEDD8 are small proteins that can be post-translationally attached to target proteins and thus modify them. However, these modifications are reversible and specific proteases can cleave off the SUMO or NEDD8 moiety. Cleavage was performed oncolumn with $0.1 \mu \mathrm{M}$ SENP1 (SUMO-protease) or $1 \mu \mathrm{M}$ NEDP1 (NEDD8-protease) (Frey and Görlich, 2014a). After binding of the protein of interest to the affinity resin, the resin was washed with buffer, the protease was added (diluted in the previously used buffer) and the column was plugged. Incubation was performed at $4^{\circ} \mathrm{C}$ for $1-2 \mathrm{~h}$. After on-column cleavage, the cut protein was eluted in $0.5 \mathrm{ml}$ steps with buffer (without protease). The collected fractions were analyzed for their protein content using amido black staining. Protein containing fractions were pooled, the protein concentration was measured and the samples were snap-frozen in liquid nitrogen and stored at $-80^{\circ} \mathrm{C}$.

\subsubsection{Buffer Exchange}

During direct cleavage of the $\mathrm{Ni}^{2+}$ elute with TEV protease, the buffer still contains high concentrations of imidazole (see 4.2.2.3). For reverse $\mathrm{Ni}^{2+}$ chromatography this competitor has to be removed. The most efficient way is to rebuffed the elution fraction to a buffer containing no imidazole. For thus purpose a PD10 or a NAP5 column (depending on the sample volume, GE Healthcare) were used according to the manufacturers instructions. The resulting elution fractions were analyzed for their protein content using amido black staining. Protein containing fractions were pooled, the protein concentration was measured and the samples were either snap-frozen in liquid nitrogen and stored at $-80^{\circ} \mathrm{C}$ or immediately used for downstream applications.

\subsubsection{Stochiometric complex assembly using two affinity tag systems and an orthogonal protease system}

In order to obtain highly pure and stochiometric heterodimeric complexes for crystallization, a purification approach utilizing two affinity tag systems and an orthogonal protease pair was performed (Frey and Görlich, 2014b).

As shown in figure 4.1 a lysate containing both subunits of the target complex was applied to two consecutive affinity chromatography steps. The lysate was optioned by either coexpressing the two subunits or by expressing them individually and mixing the lysates prior to purification. The lysate containing a $\mathrm{H}_{14}$-brSUMO tagged subunit and a ZZ-brNEDD8 tagged subunit was first applied to a reasonable amount of $6 \% \mathrm{Ni}^{2+}$-chelate $500 \AA$ silica. 


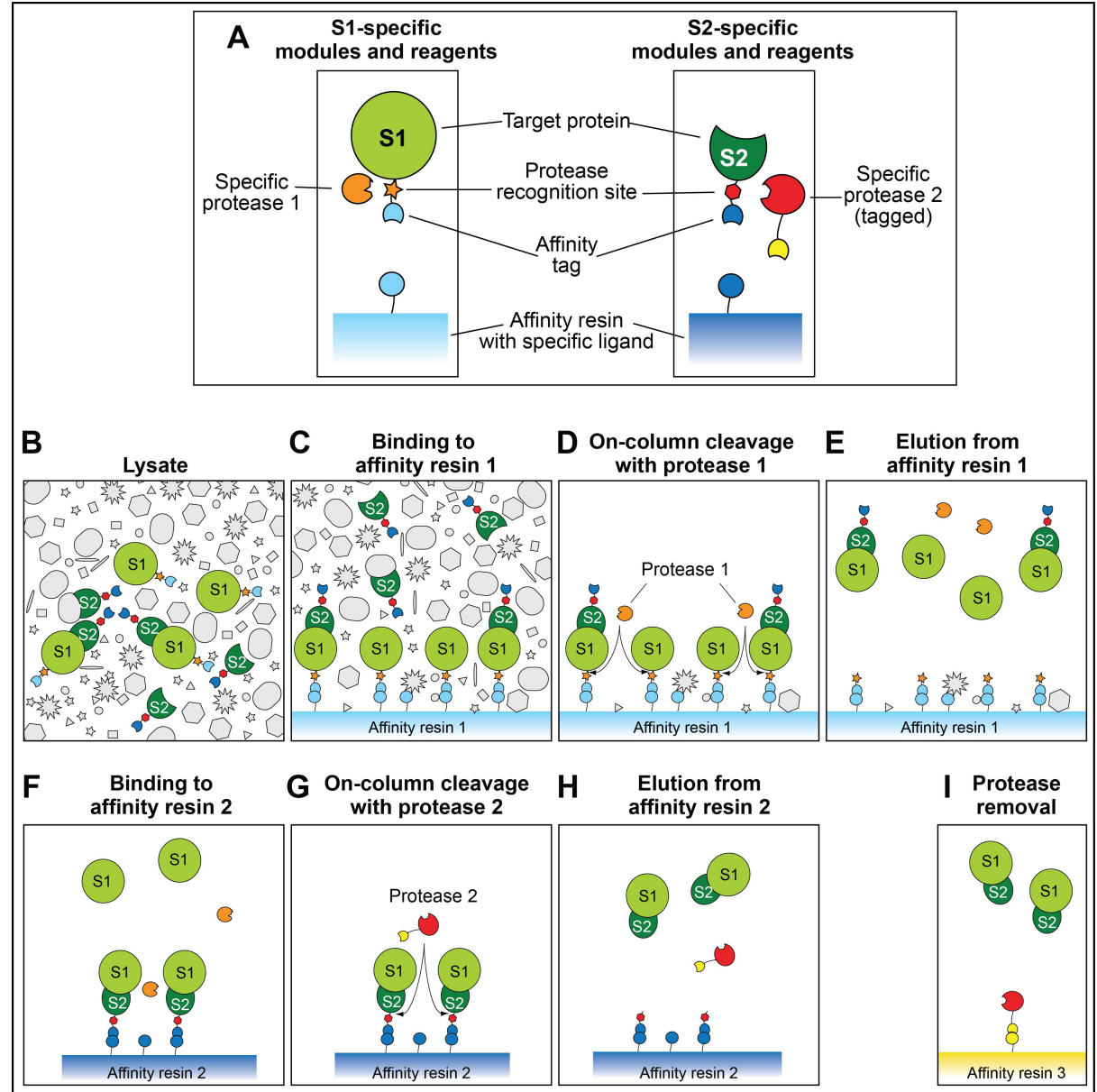

Figure 4.1: Purification of stochiometric heterodimeric complexes using two affinity tag systems and on-column cleavage (A) Description of components used in the following schemes. A lysate containing the two subunits $\mathrm{S} 1$ and $\mathrm{S} 2$ of the target complex (B) is applied to the first affinity resin, which allows binding of the affinity tag present on subunit $\mathrm{S} 1$ (C). Elution from affinity resin 1 is performed by on-column cleavage using protease 1 recognizing the protease recognition site on S1 (D). S1 subunits either alone or bound to the $\mathrm{S} 2$ subunit are eluted and subsequently bound to affinity resin 2 which binds the affinity tag present on subunit S2 (F). Surplus $S 1$ subunits pass the column without binding. Protease 2 recognizing the recognition site on the S2 subunits $(G)$ and heterodimeric, stochiometric complexes elute from affinity resin $2(\mathrm{H})$. If it is necessary for further applications to remove residual amounts of remaining protease 2 , the protease can also be applied in a tagged form and subsequently be removed by applying the elution to an affinity resin 3. The figure was extracted from Frey and Gölich (2014b)

After binding in batch for $1 \mathrm{~h}$ at $4^{\circ} \mathrm{C}$, the flow through was collected and the resin was thoroughly washed and incubated with $0.1 \mu \mathrm{M}$ SENP1 for $1 \mathrm{~h}$ at $4^{\circ} \mathrm{C}$. Elution was performed in $1 \mathrm{ml}$ steps and the resulting fractions were checked for their protein content using amid black staining. The protein containing fractions were diluted 1:5 with buffer and applied to a reasonable amount of ZpA963 2B Sepharose (ZpA963 is an affibody recognizing the ZZ tag on subunit 2; Lindborg et al., 2013). After binding in batch for $1 \mathrm{~h}$ at $4^{\circ} \mathrm{C}$, the flow through was collected and the resin was washed. Subsequently, $1 \mu \mathrm{M}$ NEDP1 protease was added to the resin recognizing the brNEDD8 stretch on the directly bound subunit. The elution was performed in $1 \mathrm{ml}$ steps and the resulting fractions were analyzed for their 
protein content. The elution should contain a highly pure, stochiometric heterodimeric complex.

Samples were taken during the entire procedure and were analyzed by SDS-PAGE.

\subsubsection{Biotinylation of proteins}

The streptavidin:biotin system is commonly used in affinity chromatography. Streptavidin molecules form homotetramers that non-covalently binds biotin with femtomolar affinity. Binding assays on streptavidin resins (silica, agarose beads or magnetic beads) require biotinylation of the bait protein in order to efficiently bind the target protein. To be able to biotinylate the protein of interest, an Avi tag was included in the expression construct, comprised of a 15 amino acid long stretch (GLNDIFEAQKIEWHE). The tag is recognized by the biotin holoenzyme synthetase BirA and the biotin moiety is covalently attached to the Avi-tag (Beckett et al., 1999).

Biotinalytion was achieved by co-expressing the protein of interest with a plasmid coding for BirA (prepared by S. Frey). Upon induction, $20 \mu \mathrm{g} / \mathrm{ml}$ biotin was added to the culture to enable efficient incorporation. In addition, biotinylation can also be performed in vitro by incubating the Avi-tag containing protein with $1 \mathrm{x}$ biotinylation buffer $(50 \mathrm{mM}$ Tris/HCl, pH 7.5, 50mM NaCl, 5mM DTT, 10mM ATP, $12.5 \mathrm{mM} \mathrm{MgCl}_{2}, 250 \mu \mathrm{M}$ biotin) and recombinantly expressed BirA enzyme in a 1:1000 ratio. The reaction takes place while incubating the sample at RT for 2-4h. After incubation, the sample has to be rebuffered to remove remaining free biotin.

\subsubsection{Size Exclusion Chromatograph (SEC)}

Size exclusion chromatography is used to separate proteins or protein complexes in solution according to their size or molecular weight. It is based on the principle that smaller molecules can enter the pores of the porous resin more efficiently than larger molecules and thus larger molecules pass the column quicker and elute earlier. For SEC either a Superdex 200 or a Superdex 75 column (Pharmacia) was used. The SD200 can separate proteins in a range from $10 \mathrm{kDa}-600 \mathrm{kDa}$, the $\mathrm{SD} 75$ separates in a range of $3 \mathrm{kDa}-70 \mathrm{kDa}$. The chromatography was performed using an ÄKTA Purifier System according to the manufacturers instructions. The column was equilibrated in the desired buffer using a default flow rate. The protein samples were ultracentrifuged at $40.000 \mathrm{rpm}$ to remove precipitates and subsequently loaded to the equilibrated column. All elution fractions were collected and analyzed by SDS-PAGE. Protein containing fractions were pooled, the protein concentration was measured and the samples were either snap-frozen in liquid nitrogen and stored at $-80^{\circ} \mathrm{C}$ or immediately used for downstream applications. 


\subsubsection{SDS PAGE}

Analysis of protein samples was performed by discontinuous sodiumdodecylsulfate polyacrylamide gel electrophoresis (SDS-PAGE) according to standard protocols (Laemmli, 1970; Sambrook and Russell, 2001). The gradient SDS-polyacrylamid gels (composition see below) were prepared by G. Kopp and J. Schünemann.

Gels were run at $50 \mathrm{~mA}$ constant current for $\sim 60-70$ minutes until the bromphenol blue dye present in the sample buffer reaches the end of the gel. Subsequently, the gels were fixed and stained by heating the gel in 3\% acetic acid and 1:100 dilution of the Coomassie stock solution (2\% (w/v) Coomassie Brilliant Blue G520 in 50\% Ethanol). Gels were subsequently destained in desalted water and finally scanned for documentation using the EPSON scanner.

$\begin{array}{llll} & \text { Heavy Gel 16\% } & \text { Light Gel 7.5 \% } & \text { Stacking Gel 4.5\% } \\ & 200 \mathrm{ml} & 200 \mathrm{ml} & 200 \mathrm{ml} \\ 2 \mathrm{M} \text { Tris pH8.8 } & 40 \mathrm{ml} & 40 \mathrm{ml} & - \\ 0.5 \mathrm{M} \text { Tris pH6.8 } & - & - & 15 \mathrm{ml} \\ \mathrm{H}_{2} \mathrm{O} & 32 \mathrm{ml} & 107 \mathrm{ml} & 68 \mathrm{ml} \\ 2 \mathrm{M} \text { sucrose } & 10 \mathrm{ml} & - & - \\ \text { Glycerol }(87 \%) & 8 \mathrm{ml} & - & - \\ 10 \% \text { SDS } & 2 \mathrm{ml} & 2 \mathrm{ml} & 1 \mathrm{ml} \\ \text { Rotiphorese Gel } 30 & 108 \mathrm{ml} & 51 \mathrm{ml} & 15 \mathrm{ml} \\ \text { TEMED } & 120 \mu \mathrm{l} & 120 \mu \mathrm{l} & 100 \mu \mathrm{l} \\ \text { APS } 10 \% & 2 \mathrm{x} 580 \mu \mathrm{l} & 2 \mathrm{x} 580 \mu \mathrm{l} & 1 \mathrm{ml}\end{array}$

\subsubsection{Western Blot}

SDS gels, Whatman papers and nitrocellulose membranes were equilibrated for 10 minutes in 1x Blotting buffer (see 4.1.7), which was also used as transfer buffer during blotting. The SDS gel was placed on a layer of Whatman paper (3.0mm, Whatman, Germany), followed by the nitrocellulose membrane. On top another layer of Whatman paper was placed and all air bubbles were carefully removed by streaking them out. The membrane was positioned such that it faces the positive pole of the blotting chamber to enable the proteins intercalated with negatively charged SDS can migrate towards the membrane. Blotting was performed at $4^{\circ} \mathrm{C}$ either for $4 \mathrm{~h}$ at $400 \mathrm{~mA}$ or over night at $100 \mathrm{~mA}$ while gently stirring.

Subsequently, the membrane was carefully removed from the chamber and blocked by incubation for at least $1 \mathrm{~h}$ with blocking buffer (4g milk powder in 100ml 1xTBS buffer). The blocking buffer was then exchanged by a milk-TBS solution containing the primary 
antibody in appropriate dilutions (1:100-1:50.000 or 1 $\mathrm{gg} / \mathrm{l})$. The membrane was incubated with the first antibody either at room temperature for $1-2 \mathrm{~h}$ or over night at $4^{\circ} \mathrm{C}$. Next, the membrane was washed with $1 \mathrm{x}$ TBS buffer (3x 10 minutes) and incubated with the secondary antibody (Goat $\alpha$-rabbit IRdye; Goat $\alpha$-mouse IRdye, Licor, USA) for 1 h at room temperature wrapped in foil to avoid any fading of the signal. After washing $3 \times 10$ minutes with $1 \mathrm{x}$ TBS, the membrane was air-dried and subsequently scanned at $700 \mathrm{~nm}$ and $800 \mathrm{~nm}$ wavelength using the Odyssey scanner (Licor, USA).

\subsubsection{Binding Assays}

Binding Assays were performed to analyze the direct interactions between two proteins. In this study, binding assays were performed on Streptavidin agarose or magnetic beads recognizing biotinylated proteins, ZZ-Affibody or IgG beads, which enables ZZ-tagged proteins to bind, and $\mathrm{Ni}^{2+}$-chelate matrix recognizing poly-histidine stretches. Binding assays were performed either with pre-purified recombinant proteins or by mixing lysates containing the expressed proteins of interest. Furthermore binding assays were performed with immobilized baits, meaning that the bait protein was immobilized to the beads first, the excess was washed away and the prey protein was subsequently provided. Alternatively, prey and bait protein were premixed before adding the affinity resin.

In general, $20 \mu \mathrm{l}$ of beads were equilibrated with $2 \mathrm{x} 500 \mu \mathrm{l}$ binding buffer $(50 \mathrm{mM}$ Tris $/ \mathrm{HCl}$, $\mathrm{pH} 7.5,2 \mathrm{mM}$ imidazole, $1 \mathrm{mM}$ DTT, $100-500 \mathrm{mM} \mathrm{NaCl}$, depending on the specific interaction to be analyzed). Next, the bait protein was added in slight excess to the beads and incubated for $1 \mathrm{~h}$ at $4^{\circ} \mathrm{C}$ while gently rotating. The flow through was collected and analyzed for its unbound protein fraction, the beads were washed $3 \mathrm{x}$ with $500 \mu$ l binding buffer prior to addition of the prey protein. The samples were incubated for $2 \mathrm{~h}$ at $4^{\circ} \mathrm{C}$ while gently rotating. Next, the flow through was collected and the beads were washed $2 \mathrm{x}$ with $500 \mu \mathrm{l}$ binding buffer, $1 \mathrm{x}$ with $500 \mu \mathrm{l}$ binding buffer while rotating for 5 minutes and finally a last wash with $500 \mu \mathrm{l}$ binding buffer. Elution was performed either direct by adding $2 \mathrm{x} 25 \mu \mathrm{l}$ SDS loading buffer (in case of streptavidin:biotin, the samples must be heated to $99^{\circ} \mathrm{C}$ during elution with SDS to release biotin from streptavidin) or by protein cleavage. $50-100 \mu \mathrm{l}$ binding buffer containing the protease were incubated with the beads for $1 \mathrm{~h}$ at $4^{\circ} \mathrm{C}$ prior to elution. All samples were analyzed by SDS-PAGE.

Additionally, some binding assays were also performed by immobilizing the bait protein to the beads and incubating these with wheat germ extract or HeLa lysate. The aim was to identify not only the direct interaction between two proteins but the interactome of a specific protein in the context of cell lysates, e.g. the interaction partners of RanQ69L in HeLa lysate.

$30 \mu \mathrm{l}$ ZZ-affibody beads were equilibrated in binding buffer $(50 \mathrm{mM}$ Tris/HCl $\mathrm{pH} 7.5$, 
$400 \mathrm{mM} \mathrm{NaCl}, 5 \mathrm{mM} \mathrm{MgAc}, 30 \mu \mathrm{M}$ GTP, $1 \mathrm{mM}$ DTT) prior to addition of $4 \mu \mathrm{M}$ Ran $\left(\mathrm{H}_{14^{-}}\right.$ ZZ-SUMOStar-RanQ69L $\mathrm{L}_{5-18)}$ ) in a total volume of $200 \mu$ l. The beads were incubated for $1 \mathrm{~h}$ at $4^{\circ} \mathrm{C}$ to enable the Ran construct to bind to the beads. Next, the flow through was collected, the beads were washed with binding buffer and $400 \mu \mathrm{l}$ HeLa lysate was added (supplemented with $5 \mathrm{mM} \mathrm{MgAc}$ and $30 \mu \mathrm{M}$ GTP). The mixture was incubated for $2 \mathrm{~h}$ at $4^{\circ} \mathrm{C}$. The flow through was collected and the beads were washed with low salt buffer (50mM Tris/HCl pH 7.5, 50mM NaCl, $5 \mathrm{mM} \mathrm{MgAc,} 30 \mu \mathrm{M}$ GTP, $1 \mathrm{mM}$ DTT). Elution was performed either by addition of $2 \times 30 \mu$ l SDS-loading buffer or by protease cleavage with $1 \mu \mathrm{M}$ SUMOStar protease (in $100 \mu \mathrm{l}$ buffer). Cleavage was performed for $2 \mathrm{~h}$ at $4^{\circ} \mathrm{C}$. All samples were analyzed by SDS-PAGE.

\subsubsection{Protein Characterization}

\subsubsection{Protein Identification by Mass Spectrometry}

Samples to be analyzed by Mass Spectrometry were loaded on 10\%precasted Bis-Tris gels (NuPAGE, Life Technologies) and stained over night with Colloidal Coomassie. Either entire lanes were analyzed by being cut in 23 equally sized slices (complete analysis) or individual bands that were carefully cut out from the gel. Subsequently, in-gel tryptic digestion and peptide extraction were performed as previously described (Shevchenko et al., 1996). The digested peptides from each band or slice were separated by reverse-phase HPLC using a Reprosil C18 column (Dr. Maisch GmbH) and eluted into the LTQ-Orbitrap XL mass spectrometer (Thermo Fisher Scientific; performed by the department of Bioanalytical Mass Spectrometry). The resulting MS/MS spectra were searched against the NCBI nonredundant database with appropriate taxonomy filters (here against Viridiplantae (green plants)) using the MASCOT v2.2 software. MASCOT search results were further analyzed using the Scaffold 3.0 software.

\subsubsection{Thermofluor}

The Thermofluor assay is a temperature-based technique to analyze the stability of either individual proteins or protein complexes. Small amounts of the protein solution are mixed with a dye that binds to hydrophobic patches and fluoresces. By increasing temperature (using a real time PCR machine, BioRad), the proteins start to unfold and thus, the fluorescence signal increases. The resulting melting curves enable to determine the melting temperature and thus the stability of the sample (Pantoliano et al., 2001; Niesen et al., 2007).

$8 \mu \mathrm{l}$ protein solution at a concentration of $0.5 \mathrm{mg} / \mathrm{ml}$ were mixed with $2 \mu$ l Sypro Orange ${ }^{\circledR}$ and transferred to a BioRad real time PCR machine for monitoring. The samples war heated from $30^{\circ} \mathrm{C}$ to $110^{\circ} \mathrm{C}$ at $1^{\circ} \mathrm{C} / \mathrm{min}$ steps. 


\subsubsection{Protein Crystallization}

Protein complexes were purified as shown in figure 4.1 and were subsequently applied to gelfiltration (20mM Tris/HCl pH7.5, 100mM NaCl, 1mM DTT) to further increase the purity. Samples were concentrated to $\sim 10 \mathrm{mg} / \mathrm{ml}$ using Amicon ${ }^{\circledR}$ Ultra Filters (Merck Milipore). The Barnase:Barstar complex was crystallized from 50mM HEPES/KOH pH7.0 or Bis/Tris phosphate-citrate pH 7.1 supplemented with 15\% PEG6000, $1.2 \mathrm{M} \mathrm{LiCl}$ and $10 \mu \mathrm{l}$ MPD (2-Methyl-2,4-pentanediol). Crystals grew at $18^{\circ} \mathrm{C}$ as small prism shaped crystals. Prior to mounting using $0.08 \mathrm{~mm}$ loops, the crystals were dehydrated using ammonium sulfate. The data was collected at the Swiss Light Source (SLS) using beam line PXII and was further processed by XDS (Kabsch, 1993). The phase was solved by molecular replacement using 1BRS (Buckle et al., 1994) as phasing model. The structure was further refined using Phenix (Adams et al., 2002) and Coot (Emsley and Cowtan, 2004). Data collection and refinement statistics are shown in table 4.5. Crystallization trails and structure solving was performed by Dr. Sergei Trakhanov.

Table 4.5: X-ray data collection and refinement statistics Barnase:Barstar complex Barnase $_{\mathrm{H} 102 \mathrm{D}}:$ Barstar $_{\mathrm{C} 42 \mathrm{~K}}$ complex was purified as shown in figure 2.24. The elution samples were concentrated to $10 \mathrm{mg} / \mathrm{ml}$ and subsequently crystallized in 50mM BTP-citrate, $1.2 \mathrm{mM} \mathrm{LiCl,} 15 \%$ PEG 6000. Crystallization trails and structure solving were performed by Dr. Sergei Trakhanov. ${ }^{*}=$ no H in calculation of No. of atoms

\begin{tabular}{|c|c|c|c|}
\hline \multicolumn{2}{|c|}{ Data Collection } & \multicolumn{2}{|c|}{ Refinement (Phenix) } \\
\hline Space Group & $\mathrm{C} 222(20)$ & Resolution $(\AA)$ & $28.6-1.95$ \\
\hline Unit cell Dimensions $(\AA)$ & $\begin{array}{c}\mathrm{a}=44.15 \mathrm{~b}=113.10 \\
\mathrm{c}=98.57\end{array}$ & No. of reflections & 16857 \\
\hline Solvent content $(\%)$ & 53.5 & Completeness (\%) & 96.3 \\
\hline Temperature (K) & 100 & $\mathrm{R} / \mathrm{R}_{\text {free }}$ & $0.215 / 0.246$ \\
\hline X-Ray Source & SLS (PXII) & $\begin{array}{l}\text { Protein molecules } \\
\text { in asymmetric unit }\end{array}$ & 1 \\
\hline Oscillation Angle $\left({ }^{\circ}\right)$ & 0.2 & No. of atoms (Protein) ${ }^{*}$ & 3220 \\
\hline Resolution $(\stackrel{\circ}{)}$ & $50-1.9$ & No. of atoms (Solvent) & 144 \\
\hline Mosaicity $\left({ }^{\circ}\right)$ & 0.26 & $\begin{array}{c}\text { R.m.s deviation from } \\
\text { ideal (Bond length }(\AA))\end{array}$ & 0.003 \\
\hline $\mathrm{R}_{\text {mean }}$ & $0.062(0.66)$ & $\begin{array}{l}\text { R.m.s deviation from } \\
\text { ideal (Bond angles }\left({ }^{\circ}\right) \text { ) }\end{array}$ & 0.77 \\
\hline$<\mathrm{I} / \sigma(\mathrm{I})>$ & $10.7(1.8)$ & $<$ B-factor $>\left(\mathrm{A}^{2}\right)$ & 30.6 \\
\hline Reflections measured & 54935 & $\begin{array}{c}\text { Ramachandran } \\
\text { statistics (favored) }\end{array}$ & $95.5 \%$ \\
\hline Reflections unique & 19121 & $\begin{array}{c}\text { Ramachandran } \\
\text { statistics (allowed) }\end{array}$ & $3.5 \%$ \\
\hline Completeness (\%) & $96.2(92.4)$ & $\begin{array}{c}\text { Ramachandran } \\
\text { statistics (outliers) }\end{array}$ & $1.0 \%$ \\
\hline Multiplicity & 2.9 & & \\
\hline Wilson B-factor $\left(\AA^{2}\right)$ & 37.3 & & \\
\hline
\end{tabular}




\subsubsection{Antibody Purification and Immunoprecipitation Experiments}

\subsubsection{Antibody Production}

The antibodies used and purified in this study were mainly raised in rabbits (see 4.1.10). By injecting recombinantly produced antigens to the rabbit, either as natively purified proteins or as inclusion bodies, the rabbit raises specific antibodies against the antigen presented. Recombinant proteins were rebuffered to a suitable buffer $(20 \mathrm{mM}$ Tris/HCl, $\mathrm{pH} 7.5,150 \mathrm{mM} \mathrm{NaCl}, 250 \mathrm{mM}$ sorbitol) and $\sim 500 \mu \mathrm{g}$ protein were adjusted to $500 \mu \mathrm{l}$ with 1x PBS and emulsioned in 50\% Freund's adjuvant complete (Sigma), 0.2\% Tween-20 and $60 \mu \mathrm{g} / \mathrm{ml}$ adjuvant peptide (Sigma). Inclusion bodies were adjusted to $500 \mu \mathrm{l}$ with $1 \mathrm{x}$ PBS. The immunization itself was performed by injecting the antigen (boost) to the rabbit every four weeks, the first bleed was taken after the second injection. Subsequently, 20ml blood were taken after each boost. These were then incubated at $37^{\circ} \mathrm{C}$ for $2 \mathrm{~h}$ followed by an over night incubation at $4^{\circ} \mathrm{C}$. Agglutination occurs during this time, thus the samples were centrifuged for $1 \mathrm{~h}$ at $7000 \mathrm{rpm}$ to remove formed aggregates. The supernatant (serum) was collected, supplemented with sodium azide and stored at $4^{\circ} \mathrm{C}$ for further use.

\subsubsection{Antibody Purification}

Prior to antibody purification, the antigen against which the antibody was raised was immobilized to a resin, e.g. maleimide Sepharose or later MADA Sepharose. Coupling occurred via the maleimide moiety on the resin and reduced cystein side chains at the C-terminus of the recombinantly expressed antigen.

$2 \mathrm{ml}$ of maleimide Sepharose were transferred to a gravity flow column and equilibrated in coupling buffer $\left(20 \mathrm{mM} \mathrm{K}_{2} \mathrm{HPO}_{4} \mathrm{pH} 7.3,200 \mathrm{mM} \mathrm{NaCl}\right)$. In parallel, the antigen was freshly reduced by incubation for 10 minutes with $10 \mathrm{mM}$ DTT and subsequently rebuffered to DTT free coupling buffer. To monitor the DTT separation, $1 \mu$ l Ellman's reagent $(10 \mathrm{mM}$ DTNB in $1 \mathrm{M}$ imidazole) was mixed with $4 \mu \mathrm{l}$ of protein solution.

The antigen was then added to the equilibrated resin at concentrations of 5-10mg protein per $\mathrm{ml}$ matrix, mixed immediately and gently rotated for 30 minutes at room temperature. After incubation, the flow through was collected and analyzed for its protein content. The antigen coupled matrix was quenched with $0.1 \mathrm{M} \beta$-mercaptoethanol for 20 minutes at RT prior to thorough washing with $1 \mathrm{x}$ PBS. The columns were stored at $4^{\circ} \mathrm{C}$ until further use. The capacity of the antigen column towards the antibodies in the rabbit sera vary greatly depending in the antibody titer. As a standard, $25 \mathrm{ml}$ sera were used to be purified via $2 \mathrm{ml}$ antigen matrix. The sera was mixed 1:1 with $1 \mathrm{xPBS}$ and centrifuged at $10.000 \mathrm{rpm}$ for 10 minutes to remove any aggregates. The matrix was washed twice with $1 x$ PBS, preeluted with $2 \times 10 \mathrm{ml} 0.1 \mathrm{M}$ glycine $\mathrm{pH} 2.2$ and subsequently equilibrated in $1 \mathrm{x}$ PBS. The 
sera was then applied twice to the antigen columns and the flow through was collected. After washing the column with $1 \mathrm{xPBS}$, elution was performed using $3 \mathrm{x} 5 \mathrm{ml} 0.1 \mathrm{M}$ glycine, $\mathrm{pH} 2.2$. To neutralize the eluate, it was immediately mixed with $1 \mathrm{ml} 1 \mathrm{M} \mathrm{K}_{2} \mathrm{HPO}_{4} \mathrm{pH}$ 7.3. The antibody suspension was precipitated with $30 \mathrm{ml} 4 \mathrm{M}$ ammonium sulfate over night at $4^{\circ} \mathrm{C}$. Next, the suspension was centrifuged for $1 \mathrm{~h}$ at $10.000 \mathrm{rpm}$ and most of the supernatant was carefully removed. The antibody pellet was resuspended in the remaining supernatant and stored at $4^{\circ} \mathrm{C}$.

Input, flow through and elution samples were subsequently analyzed by Western Blot using a 1:100 dilution of the input and flow through and $1 \mu \mathrm{g} / \mathrm{ml}$ of the eluted antibody.

To obtain highly pure antibodies, the sera was not only purified via the antigen coupled column. In addition, the sera passed a column to which E. coli proteins were immobilized and a column containing immobilized fusion tags if these were injected to the rabbit during immunization. The aim was to pre-deplete any non-specific antibodies prior to possible antigen binding.

\subsubsection{Covalent Coupling of Antibodies to ProteinA}

In order to perform immunoprecipitation (IP) experiments, the antibodies raised against the desired target were covalently coupled to ProteinA immobilized on Sepharose (prepared in the lab, recombinantly expressed ProteinA was immobilized to maleimide Sepharose or MADA Sepharose via reduced cysteins).

$150 \mu \mathrm{g}$ antibody were suspended in $400 \mu \mathrm{l}$ 2xPBS. In parallel, $50 \mu \mathrm{l}$ ProteinA-Sepharose were pre-eluted with $500 \mu \mathrm{l} 0.1 \mathrm{M}$ glycine $\mathrm{pH} 2.2$ and subsequently equilibrated with $2 \mathrm{x}$ $500 \mu \mathrm{l} 1 \mathrm{xPBS}$. Next, the antibody solution was transferred to the beads and incubated for $1 \mathrm{~h}$ at $4^{\circ} \mathrm{C}$ while gently rotating. After incubation, the flow through was collected by low speed centrifugation (800-1000rpm) and the beads were washed $3 \mathrm{x}$ with $500 \mu \mathrm{l} 2 \mathrm{xPBS}$ followed by $3 \mathrm{x}$ wash with $200 \mathrm{mM} \mathrm{NaPO}_{4} \mathrm{pH} 8.0$. The beads were then gently resuspended in $500 \mu \mathrm{l} 0.02 \%$ glutaraldehyde (GA) for cross linking the antibodies to the ProteinA and incubated for $1 \mathrm{~h}$ at room temperature while rotating. Glutaraldehyde was removed by gentle centrifugation and the beads were resuspended in $400 \mu \mathrm{l} 3 \mathrm{mg} / \mathrm{ml} \mathrm{NaCNBH} 3$ (solubilized in $50 \mathrm{mM} \mathrm{NaPO}_{4} \mathrm{pH} 7.5$ ) in order to reduce the formed Schiff bases to stable amino bonds. After incubated for $1 \mathrm{~h}$ at room temperature, the flow through was removed and the beads were resuspended in $500 \mu \mathrm{l} 3 \mathrm{mg} / \mathrm{ml} \mathrm{NaCNBH} 3$ (solubilized in $50 \mathrm{mM} \mathrm{NaPO}_{4} \mathrm{pH} 7.5$, $50 \mathrm{mM}$ glycine $\mathrm{pH} 7.5$ ) and incubated for 20 minutes to quench the remaining aldehyde groups. Finally, the beads were washed $2 \mathrm{x}$ with $500 \mu \mathrm{l}$ 1xPBS and stored in $20 \%$ ethanol at $4^{\circ} \mathrm{C}$ until further use. 


\subsubsection{Immunoprecipitation Experiments}

Immunoprecipitation is a technique to pull out desired target proteins and their interaction partners from a lysate, e.g. wheat germ extract, by specific antibodies against the target protein.

The specific antibodies were coupled on ProteinA Sepharose (see 4.2.5.3). 10 $\mu$ l antibody beads were transferred to a fresh Mobicol column (Mobitec GmbH, Germany) and preeluted with $2 \mathrm{x} 25 \mu \mathrm{l} 0.1 \mathrm{M}$ glycine $\mathrm{pH} 2.2$ and subsequently equilibrated in $500 \mu \mathrm{l}$ buffer (50mM Tris pH7.5, 500mM NaCl, $5 \mathrm{mM} \mathrm{MgAc})$. Next, the beads were incubated with $500 \mu \mathrm{l}-2 \mathrm{ml}$ wheat germ extract (depends on the amount of the target protein in the lysate) at $4^{\circ} \mathrm{C}$ while gently rotating. After removing the unbound fraction, the beads were washed $3 \mathrm{x}$ with $500 \mu \mathrm{l}$ buffer followed by washing once with $500 \mu \mathrm{l}$ buffer $+0.2 \%$ TritonX100. After washing once more with $500 \mu \mathrm{l}$ buffer, the bound proteins were eluted with $2 \mathrm{x}$ $50 \mu \mathrm{l} 0.1 \mathrm{M}$ glycine pH2.2 into a fresh Eppendorf tube containing $20 \mu \mathrm{l} 1 \mathrm{M}$ Tris/HCl pH8.8 for immediate neutralization. The elute was then precipitated with trichloroacetic acid (TCA) and the resulting pellet was carefully resuspended in 15-50 $\mu$ l SDS loading buffer (depending on the size of the pellet). Elution samples were analyzed by SDS-PAGE. The beads were washed once more with $500 \mu \mathrm{l} 1 \mathrm{xPBS}$ and stored at $4^{\circ} \mathrm{C}$.

\subsubsection{In vitro Translation}

In vitro translation systems are used to produce proteins of interest in a cell-free environment. Mainly eukaryotic in vitro translation systems are of great interest as they are able to produce eukaryotic proteins in an eukaryotic surrounding, providing chaperones, modifying enzymes and potential assembly factors. These are lacking when expressing eukaryotic proteins in E.coli.

Moreover, eukaryotic in vitro translation systems enable to monitor and analyze (eukaryotic) protein synthesis and to massively manipulate this process. In an in vivo system these manipulations would often lead to severe or even lethal phenotypes.

In this study two eukaryotic in vitro translation systems were used: the wheat germ extract to study the necessity of certain translation initiation factors, and the rabbit reticulocyte lysate system (RRL) to study the effects of nuclear transport factors on overall translation rates.

\subsubsection{1 mRNA preparation}

In oder to perform in vitro translation using the wheat germ extract, mRNA of the reporter was produced. The T7 RiboMAX ${ }^{\mathrm{TM}}$ Kit (Promega) was used for initial mRNA production from a linearized DNA plasmid, the ScriptCap m7G Capping System (Biozyme) for subsequent capping of the mRNA. 
$5 \mu \mathrm{g}$ T38 plasmid $\left(\mathrm{H}_{21}\right.$-TEV-Firefly Luciferase, produced by Dr. Cathrin Enke) was linearized using EcoRI restriction enzyme. After purification of the DNA using the Zymoclean Gel DNA recovery Kit, $2 \mu \mathrm{g}$ DNA were incubated with $20 \mu \mathrm{l}$ T7 5x Buffer, $7.5 \mu \mathrm{l}$ of each rNTPs and $10 \mu \mathrm{l}$ T7 Enzyme Mix (all components of the T7 RiboMAX ${ }^{\mathrm{TM}}$ Kit). The reaction was filled up to $100 \mu \mathrm{l}$ with RNAse free water and incubated at $37^{\circ} \mathrm{C}$ for 4h. The mRNA was subsequently purified by a phenol-chloroform extraction and ethanol precipitation. $80 \mu \mathrm{g}$ recovered mRNA were heated to $65^{\circ} \mathrm{C}$ for 10 minutes and placed on ice immediately. The sample was mixed with $10 \mu$ l 10x ScriptCap Capping Buffer, $10 \mu \mathrm{l}$ 10mM GTP and $0.5 \mu \mathrm{l} 20 \mathrm{mM}$ SAM stock solution. After mixing, $3 \mu \mathrm{l}$ ScriptGuard RNase inhibitor and $5 \mu \mathrm{l}$ ScriptCap Capping Enzyme was added and the reaction was incubated at $37^{\circ} \mathrm{C}$ for $2 \mathrm{~h}$. Subsequently, $5 \mu \mathrm{l}$ ScriptCap 2'-O-Methyltransferase were added, followed by another incubation at $37^{\circ} \mathrm{C}$ for $1 \mathrm{~h}$. After a final phenol-chloroform extraction, the capped mRNA were aliquoted in small fractions and stored at $-80^{\circ} \mathrm{C}$.

\subsubsection{In vitro Wheat Germ Translation System}

The in vitro wheat germ extract translation assay allows to monitor the translation efficiency of the system towards a given reporter under certain conditions. The wheat germ extract (WGE) was mixed with mRNAs coding for the reporter (e.g. Firefly luciferase), amino acids and an energy regenerating system. After successful translation, the amount of produced reporter proteins was read out by the luciferase activity assay. The luminescence measured can be directly correlated to the efficiency of translation under the given conditions.

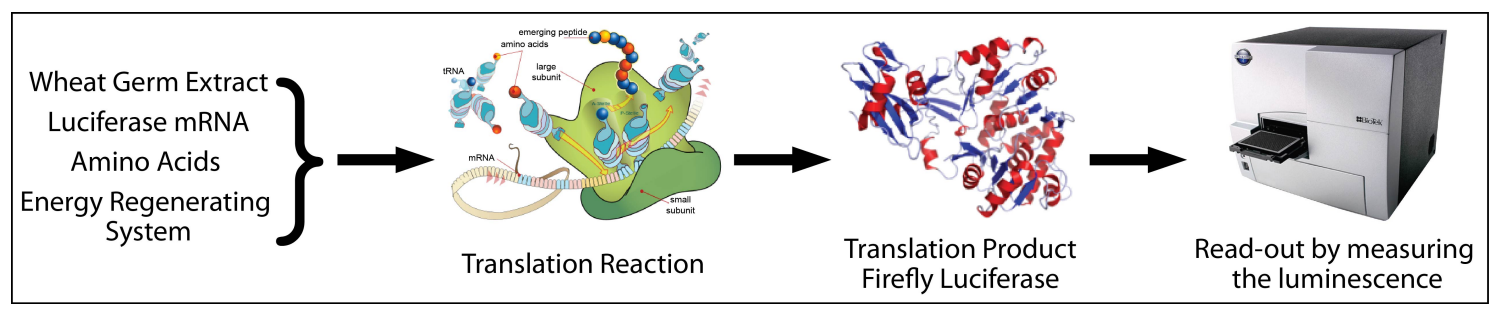

Figure 4.2: In vitro wheat germ translation assay In-house produced wheat germ extracts are mixed with mRNA encoding for Firefly Luciferase, amino acids and an energy regenerating system and incubated at $27^{\circ} \mathrm{C}$ for 90 minutes to enable efficient translation of the firefly luciferase reporter construct. The amount of translated protein is estimated by measuring the emitted light during the luciferase activity assay using a BioTek Synergy H4 Plate Reader.

The wheat germ extracts (WGE) used in this study were produced in house (for detailed protocol see Enke, 2010). The WGEs were stored at $-150^{\circ} \mathrm{C}$ in $20 \mathrm{mM}$ Hepes/KOH pH7.8, $115 \mathrm{mM} \mathrm{KAc}, 2.5 \mathrm{mM} \mathrm{MgAc}, 4 \mathrm{mM}$ DTT and $\%$ glycerol as freeze protectant. Prior to translation, the WGE was supplemented with $1.2 \mathrm{mM}$ ATP, $0.25 \mathrm{mM}$ GTP, $16 \mathrm{mM}$ creatine phosphate and $100 \mu \mathrm{g} / \mathrm{ml}$ creatine kinase.

The wheat germ translation assay was comprised out of the following: 


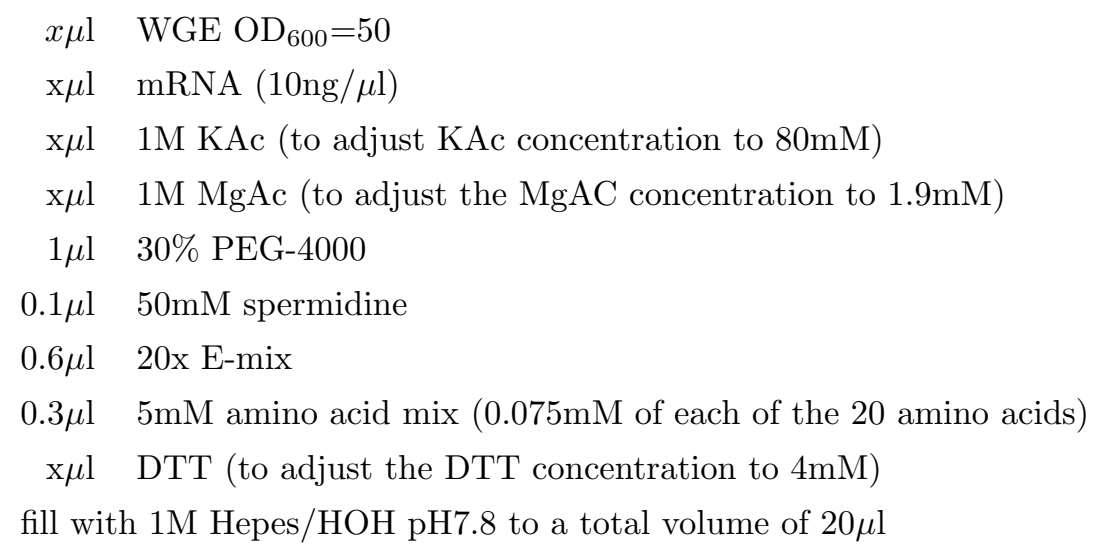

For efficient translation, all components but the mRNA were pipetted on ice and thoroughly mixed to homogeneity. The mRNA was added last and mixing was performed as gentle as possible to avoid rupture of the mRNA. The reaction was incubated at $27^{\circ} \mathrm{C}$ for 90 minutes. After incubation, the reaction was stopped by adding 5mM EDTA and subsequently $10 \mu \mathrm{l}$ of the reaction were mixed with $10 \mu \mathrm{l}$ of water and the luciferase activity assay was performed. The assay is based on the conversion of luciferin to oxyluficerin. The enzymatic reaction was started by adding $80 \mu \mathrm{l}$ of the injection buffer $(125 \mu \mathrm{M}$ luciferin, $15 \mathrm{mM} \mathrm{MgSO}_{4}, 20 \mathrm{mM}$ glycyl-glycin, $5 \mathrm{mM}$ ATP, $1 \mathrm{mM}$ DTT, ddH $\mathrm{d}_{2} \mathrm{O}$ ) to the translation sample in an automated manner and bioluminescence was measured using the BioTek Synergy H4 Plate Reader.

\subsubsection{In vitro Rabbit Reticulocyte Lysate (RRL) Translation System}

In this study, the in vitro rabbit reticulocyte lysate was used to analyze the effect of nuclear transport factors on overall translation rates. For this purpose, the TNT ${ }^{\circledR}$ Coupled Reticulocyte Lysate Systems (Promega) was used. As reporters, plasmids were constructed containing from - N-terminus to C-terminus - a 3x-FLAG tag, a poly-serine or a polyproline stretch, a short methionine/serine rich stretch for proper ${ }^{35} \mathrm{~S}-\mathrm{Met}$ incorporation and the reporter protein, either the firefly luciferase or parts of the Nup98 FG domain.

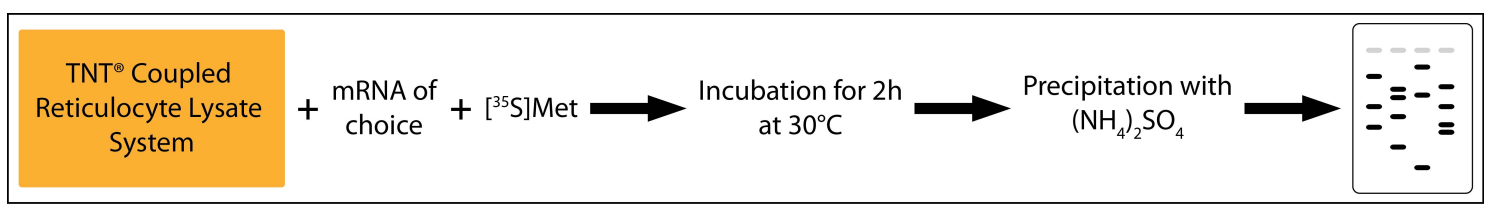

Figure 4.3: In vitro translation using rabbit reticulocyte lysate The in vitro translation assay uses the TNT ${ }^{\circledR}$ Coupled Reticulocyte Lysate System provided by Promega. The lysate is supplemented with the plasmid coding for the reporter construct and ${ }^{35} \mathrm{~S}$ Met. After incubation for $2 \mathrm{~h}$ at $30^{\circ} \mathrm{C}$, the reaction is precipitated using ammonium sulfate to remove hemoglobin. The samples are then loaded on SDSPAGE and the resulting gel is visualized by radiography. 
The translation reaction was comprised out of the following:

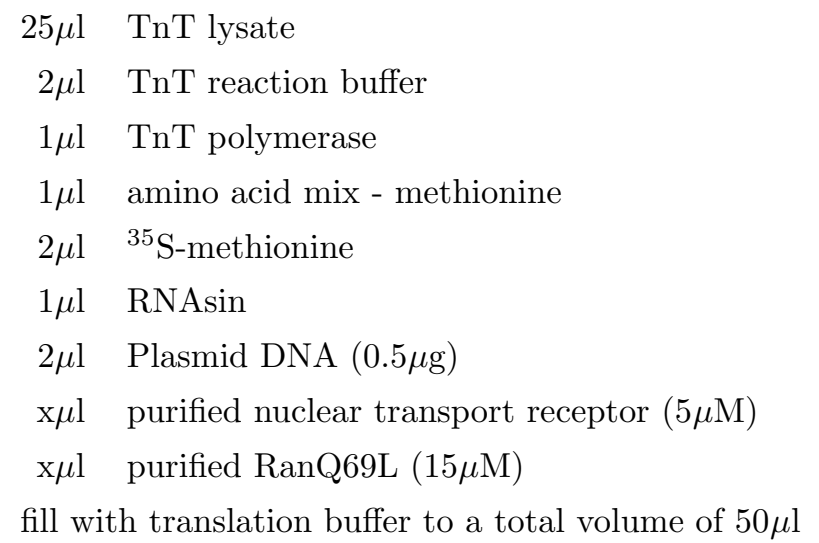

The translation buffer was comprised of the following: $50 \mathrm{mM}$ Tris/ $\mathrm{HCl} \mathrm{pH} 7.5,150 \mathrm{mM}$ $\mathrm{NaCl}, 1 \mathrm{mM}$ DTT, $250 \mathrm{mM}$ sucrose.

The reaction was incubated for 90 minutes at $30^{\circ} \mathrm{C}$ and was subsequently precipitated with a mixture of $200 \mu \mathrm{l} 1 \mathrm{xPBS}$ and $500 \mu \mathrm{l} 4 \mathrm{M}$ ammonium sulfate to remove most of the hemoglobin present in the reticulocyte lysate. The samples were incubated over night at $4^{\circ} \mathrm{C}$ and were then centrifuged for 10 minutes at $15.000 \mathrm{rpm}$. The supernatant was carefully removed and the pellet was resuspended in $40 \mu \mathrm{l}$ SDS loading buffer. The gel was subsequently incubated in a fixation solution $\left(50 \% \mathrm{MeOH}, 40 \% \mathrm{H}_{2} \mathrm{O}, 10 \%\right.$ acetic acid) and carefully dried. The dried gel was then applied to a radiographic film. After over night incubation, the film was scanned using the Fujifilm FLA7000 scanning device.

\subsubsection{Matrix Characterization}

\subsubsection{Direct coupling}

MADA is a proprietary chemistry on beads developed in our lab, which is able to form a covalent link with the thiol groups of reduced cysteins. To test the specificity of the matrix, mCherry and $\mathrm{mCherry}_{\text {cys }}$ were expressed, purified and freshly reduced with $10 \mathrm{mM}$ DTT. Remaining DTT was subsequently removed by buffer exchange. $50 \mu$ l of the MADA resin were incubated with $5 \mu \mathrm{M}$ mCherry or mCherry cys $_{\text {in }} 50 \mathrm{mM}$ Tris/HCl, $\mathrm{pH} 7.5,300 \mathrm{mM}$ $\mathrm{NACl}$ in a total volume of $200 \mu \mathrm{l}$. After incubation for $1 \mathrm{~h}$ at $4^{\circ} \mathrm{C}$, the flow through was collected and the beads were washed. The process was documented by taking pictures of the Input fractions, the beads after incubation, the flow through fraction and the beads after washing.

Besides specificity, the binding capacity of proteins with reduced cysteins towards the resin was analyzed. Either Protein $\mathrm{A}_{\mathrm{cys}}$ or Streptavidin $_{\mathrm{cys}}$ were added in increasing concentrations $(0-8 \mu \mathrm{g} / \mu \mathrm{l}$ beads) to a constant amount of MADA beads. The Input and Flow 
through fractions were measured for their protein content at $A_{280 n m}$ and analyzed by SDS-PAGE.

\subsubsection{Indirect pulldowns}

ProteinA MADA Sepharose was analyzed for its ability to specifically pull out IgG molecules from rabbit sera. Increasing amounts of ProteinA $(0-8 \mu \mathrm{g} / \mu \mathrm{l}$ beads) were coupled to a constant amount of MADA beads $(20 \mu \mathrm{l})$. Next, the resin was incubated with constant volumes of rabbit sera $(200 \mu \mathrm{l})$. The elution was performed by SDS-loading buffer and analyzes was performed by SDS-PAGE.

The streptavidin MADA Sepharose was analyzed for the ability to pull out biotinylated baits from E.coli lysate. Therefor, streptavidin was covalenty attached to the resin, which was subsequently incubated with E.coli lysate supplemented with $10 \mu \mathrm{M}$ biotinylated bait (ataxin or Nup98 anchor domain). Elution was performed at $99^{\circ} \mathrm{C}$ with SDS loading buffer. Analysis was performed by SDS-PAGE.

To pull out large complexes from e.g. the wheat germ extract, a "binding construct" $\left(\mathrm{H}_{14^{-}}\right.$ ZZ domain-SUMOStar-Cys) was coupled to the resin. In parallel, the WGE was incubated with IgGs against the target complex (here eIF3 complex and $\alpha$-eIF3c/d/m). After washing off uncoupled binding constructs from the resin, it was mixed with the IgG supplemented WGE for $1 \mathrm{~h}$ at $4^{\circ} \mathrm{C}$ to allow binding. After thorough washing, the elution was performed using $1 \mu \mathrm{M}$ SUMOStar protease (S.c.Ulp1). The elution factions were analyzed by SDS-PAGE. To remove the remaining binding construct $\left(\mathrm{H}_{14}-\mathrm{ZZ}\right.$ domain-SUMOStar), the elution fraction was run over MADA matrix coupled to ZZ-affibody (ZpA963). The remaining binding construct should bind the resin, whereas the complex of interest should not bind the resin and thus should be found in the flow through fraction. All steps were analyzed by SDS-PAGE.

\subsubsection{Phage Display}

Phage Display in an in vitro selection technique by which proteins with desired properties can be extracted from a great mixture of different variants. Therefore, a library of the protein of interest is cloned into phagemids such, that it is fused to the phage coat protein pIII. New phage particles display the encoded protein at their tip as fusion construct to pIII. By binding assays, beneficial variants of the protein of interest are accumulated, containing not only the protein but also the underlying sequence encoded on the phagmid. Hence, this technique enables a direct link between genotype and phenotype.

Standard phage display methods were performed according to Clackson and Lowman (2004) with modifications described in the following section. 


\subsubsection{Library construction}

To introduce random mutations to the protein of interest, error-prone PCR was performed using the JBS Error-Prone Kit (Jena Bioscience). The PCR reaction was performed according to the manufacturers instruction. After purifying the PCR reaction using the MSB Spin PCRapace Kit, the elute was used as template for a subsequent PCR reaction to amplify the DNA fragments. PCR primers contained enzymatic restriction sites by which the resulting fragments were ligated into the display vectors (pelB signal sequenceMCS-HA tag-pIII minor coat protein, prepared by T. Pleiner). After determination of the transformation efficiency of the library, the library was transformed into TG1 E.coli cells to reach a library size of $1 \times 10^{7}$. The transformed cells were incubated for $1 \mathrm{~h}$ at $37^{\circ} \mathrm{C}$ in recovery medium and further incubated over night in $2 \mathrm{YT}$ medium supplemented with ampicillin and $2 \%$ glucose.

The cells were carefully harvested after incubation and supplemented with $15 \%$ glycerol and stored at $-80^{\circ} \mathrm{C}$ till further use.

\subsubsection{Bacteriophage harvest and purification}

Cells transformed with the library of interest were further used to produce bacteriophages displaying the different protein variants as fusion with the pIII coat protein. 100-fold more cells than the library size (here $1 \times 10^{7}$ ) were grown in 2 YT Medium (+Ampicillin and $2 \%$ glucose) to an $\mathrm{OD}_{600}=0.8$ at $37^{\circ} \mathrm{C}$. When $\mathrm{OD}_{600}=0.8$ was reached, the cultures were infected with a 20-fold surplus of helper-phage (M13KO7, prepared by T. Pleiner) over cells and incubated for 30 minutes at $37^{\circ} \mathrm{C}$ without shaking to allow infection. The cells were subsequently harvested by centrifugation for 10 minutes at $4^{\circ} \mathrm{C}$ and the supernatant containing excess of helper-phages was discarded. The remaining pellet was resuspended in $250 \mathrm{ml} 2 \mathrm{YT}$ media containing ampicillin and kanamycin and incubated over night at $37^{\circ} \mathrm{C}$. Next, the cultures were centrifuged for 10 minutes at 7000rpm and the supernatant was mixed with pre chilled buffer $(20 \%$ PEG-8000, $2.5 \mathrm{M} \mathrm{NaCl}$ ) in a 1:5 ratio (buffer: supernatant). The mixture was incubated on ice for at least 30 minutes to allow precipitation. The samples were then centrifuged for 15 minutes at 4000rpm and the supernatant was carefully removed. The resulting white phage pellet was resuspended in $20 \mathrm{ml} 50 \mathrm{mM}$ Tris/ $\mathrm{HCl} \mathrm{pH7.5,} \mathrm{300mM} \mathrm{NaCl} \mathrm{and} \mathrm{centrifuged} 10$ minutes at $15.000 \mathrm{~g}$ to remove all remaining cells and aggregates. The supernatant was mixed with 5ml 20\%PEG-8000, 2.5M $\mathrm{NaCl}$ for 30 minutes on ice for precipitation. The samples were centrifuged at $2200 \mathrm{rpm}$ for 15 minutes and the pellet was gently resuspended in $3-5 \mathrm{ml} 50 \mathrm{mM}$ Tris/ $\mathrm{HCl} \mathrm{pH} 7.5$, $300 \mathrm{mM} \mathrm{NaCl}$. After the pellet was completely resuspended, the suspension was filled in $2 \mathrm{ml}$ Eppendorf tubes and centrifuged at $13.000 \mathrm{rpm}$ for 10 minutes. The supernatant was pooled and the phage concentration was measured with the following formula: 


$$
\text { phages } / \mathrm{ml}=\frac{\left(A_{269}-A_{320}\right) \cdot 6 \times 10^{16}}{\text { numberofbases } / \text { phagemid }}
$$

The phages were supplemented with $0.1 \%$ BSA and stored at $4^{\circ} \mathrm{C}$ for further use.

\subsubsection{Phage selection}

The selection procedure can vary greatly depending on the interaction between the bait and the phage itself and the properties to select for. The following describes a very general selection procedure.

1mg magnetic streptavidin beads (Dynabeads Streptavidin T1, Invitrogen) were washed twice with $500 \mu \mathrm{l}$ wash and block buffer (WBB, $50 \mathrm{mM}$ Tris/HCl pH 7.5, 300mM NaCl, $0.1 \% \mathrm{BSA}$ ) and incubated in $1 \mathrm{ml} \mathrm{WBB}$ for at least 30 minutes. In parallel, $2 \mathrm{ml}$ Eppendorf tubes were blocked with $2 \mathrm{ml}$ WBB buffer for at least 30 minutes. To decrease the background binding of the displaying phages to the beads, the phage pool used during the selection was pre-depleted by adding the phages in a total volume of $1 \mathrm{ml}$ WBB to $1 \mathrm{mg}$ magnetic streptavidin beads and incubated for at least 30 minutes. The amount of phages used per selection reaction was usually $1 \times 10^{12}$.

The binding of bait and phages can either be performed in solution or by immobilizing the bait to the magnetic beads beforehand. When performing in solution binding, the bait is diluted to the favored concentration in $\mathrm{WBB}$ and then mixed with the pre-depleted phages to a total volume of $1 \mathrm{ml}$ WBB and incubated for $1-2 \mathrm{~h}$ while gently rotating. Subsequently, the mixture was added to $1 \mathrm{mg}$ pre-blocked magnetic beads and further incubated for 10 minutes while rotating.

When immobilizing the bait to the beads beforehand, the appropriate amount of bait protein was diluted in a total volume of $1 \mathrm{ml}$ WBB and incubated with $1 \mathrm{mg}$ magnetic beads for 30 minutes while gently rotating. After binding, the flow through was discarded and the beads were washed twice with $1 \mathrm{ml}$ WBB to remove any non-bound bait. Subsequently, the phages were added to the beads in a total volume of $1 \mathrm{ml}$ WBB and incubate while rotating for $1 \mathrm{~h}$.

After binding, the beads were thoroughly washed in subsequent steps with WBB and WBB supplemented with $0.1 \%$ Tween-20. After individual washing steps, the tubes were changed to remove all phages sticking to the tube walls.

Elution was performed by protease cleavage. The bait constructs were designed such that the spacer between the biotin moiety and the actual bait protein contained a protease cleavage site. The wash buffer was carefully removed and $200 \mu \mathrm{l}$ WBB supplemented with the corresponding protease were added to the beads and incubated for at least 30 minutes while rotating. The supernatant containing the cleaved off phage:bait complexes was carefully removed and used for re-infection and iteration of the eluted phages.

For reinfection, $190 \mu \mathrm{l}$ of the eluted phages were used to infect $20 \mathrm{ml}$ of exponentially grow- 
ing TG1 E.coli cells. The cultures were incubated for 30 minutes at $37^{\circ} \mathrm{C}$ without shaking and subsequently diluted with $80 \mathrm{ml} 2 \mathrm{YT}$ media containing ampicillin and $2 \%$ glucose. The cultures were incubated for 30 minutes at $37^{\circ} \mathrm{C}$ while shaking prior to infection with $10^{12}$ M13KO7 helper-phages. After another 30 minutes incubation at $37^{\circ} \mathrm{C}$ without shaking, the cultures were centrifuged for 10 minutes at $4000 \mathrm{rpm}$, the pellet was resuspended in 200ml 2YT media containing ampicillin and kanamycin and the cultures were incubated over night at $37^{\circ} \mathrm{C}$.

To analyze the enrichment of phages and the selection rate, the eluted phages were in addition titrated. The remaining $10 \mu \mathrm{l}$ of eluted phages were mixed with $90 \mu \mathrm{l} 2 \mathrm{YT}$ media containing $2 \%$ glucose. A 1:10 dilution series $\left(10^{-1}-10^{-8}\right)$ was prepared and $10 \mu \mathrm{l}$ of each dilution were mixed with $90 \mu$ l exponentially growing TG1 E.coli cells. The samples were incubated at $37^{\circ} \mathrm{C}$ for 30 minutes and subsequently, $10 \mu$ l of cells were plated on ampicillin agar plates containing $2 \%$ glucose. The agar plates were incubated over night at $37^{\circ} \mathrm{C}$. In addition, $50 \mu \mathrm{l}$ of the exponentially growing TG1 cells were plated on ampicillin containing agar plates to check for possible contaminations.

To be able to sequence and clone the phagmids coding for selected binders, $80 \mu \mathrm{l}$ of the cells infected with $10^{-1}$ eluted phages were incubated over night at $37^{\circ} \mathrm{C}$ in LB media containing $2 \%$ glucose. The phagmids were subsequently purified by standard DNA preparation (see 4.2.1.11).

\subsubsection{Analysis of Binders}

Phages displaying potential binders to the bait protein were enriched during the phage selection procedure. The pool of binding phages was used to infect TG1 cells. These were then used as starting culture for plasmid preparation, leading to a DNA preparation containing the phagmids encoding the sequence of the bait binding proteins. The DNA was subsequently cloned into expression vectors (here $\mathrm{H}_{14}$-brSUMO-MCS) using standard restriction enzymes. After ligation, the cells were plated on agarose plates containing the appropriate antibiotics. 96 single colonies from different round of selection were picked after over night incubation at $37^{\circ} \mathrm{C}$ and send for sequencing (performed by GATC Biotech). After analysis of the sequences, different classes of binders can be defined and representatives of each class are expressed in E.coli and characterized. 


\section{Bibliography}

Adams, P., Grosse-Kunstleve, R., Hung, L., Ioerger, T., McCoy, A., Moriarty, N., Read, R., Sacchettini, J., Sauter, N., and Terwilliger, T. (2002). PHENIX: building new software for automated crystallographic structure determination. Acta Crystallogr D Biol Crystallogr, 58(Pt 11):1948-1954.

Akiyoshi, Y., Clayton, J., Phan, L., Yamamoto, M., Hinnebusch, A., Watanabe, Y., and Asano, K. (2001). Fission yeast homolog of murine Int-6 protein, encoded by mouse mammary tumor virus integration site, is associated with the conserved core subunits of eukaryotic translation initiation factor 3. J Biol Chem, 276(13):10056-10062.

Alkalaeva, E., Pisarev, A., Frolova, L., Kisselev, L., and Pestova, T. (2006). In vitro reconstitution of eukaryotic translation reveals cooperativity between release factors eRF1 and eRF3. Cell, 125(6):1125-1136.

Allfrey, V. (1954). Amino acod incoorporation by isolated thymus nuclei. I. The role of desoxyribonucleic acid in protein synthesis. Proc Natl Acad Sci U S A, 40(10):881-885.

Asano, K., Clayton, J., Shalev, A., and Hinnebusch, A. (2000). A multifactor complex of eukaryotic initiation factors, eIF1, eIF2, eIF3, eIF5, and initiator tRNA(Met) is an important translation initiation intermediate in vivo. Genes Dev, 14(19):2534-2546.

Asano, K., Phan, L., Anderson, J., and Hinnebusch, A. (1998). Complex formation by all five homologues of mammalian translation initiation factor 3 subunits from yeast Saccharomyces cerevisiae. J Biol Chem, 273(29):18573-18585.

Asano, K., Vornlocher, H., Richter-Cook, N., Merrick, W., Hinnebusch, A., and Hershey, J. (1997). Structure of cDNAs encoding human eukaryotic initiation factor 3 subunits. Possible roles in RNA binding and macromolecular assembly. J Biol Chem, 272(43):27042-27052.

Axén, R. and Ernback, S. (1971). Chemical fixation of enzymes to cyanogen halide activated polysaccharide carriers. Eur J Biochem, 18(3):351-360.

Banaszynski, L., Liu, C., and Wandless, T. (2005). Characterization of the FKBP.rapamycin.FRB ternary complex. J Am Chem Soc, 127(13):4715-4721.

Beckett, D., Kovaleva, E., and Schatz, P. (1999). A minimal peptide substrate in biotin holoenzyme synthetase-catalyzed biotinylation. Protein Sci, 8(4):921-929.

Benne, R. and Hershey, J. (1976). Purification and characterization of initiation factor IF-E3 from rabbit reticulocytes. Proc Natl Acad Sci U S A, 73(9):3005-3009.

Bohnsack, M., Regener, K., Schwappach, B., Saffrich, R., Paraskeva, E., Hartmann, E., and Görlich, D. (2002). Exp5 exports eEF1A via tRNA from nuclei and synergizes with other transport pathways to confine translation to the cytoplasm. EMBO J, 21(22):6205-6215. 
Bonner, W. (1975). Protein migration into nuclei. II. Frog oocyte nuclei accumulate a class of microinjected oocyte nuclear proteins and exclude a class of microinjected oocyte cytoplasmic proteins. J Cell Biol, 64(2):431-437.

Brogna, S., Sato, T., and Rosbash, M. (2002). Ribosome components are associated with sites of transcription. Mol Cell, 10(4):93-104.

Browning, K., Gallie, D., Hershey, J., Hinnebusch, A., Maitra, U., Merrick, W., and Norbury, C. (2001). Unified nomenclature for the subunits of eukaryotic initiation factor 3. Trends Biochem Sci, 26(5)(5):284.

Buckle, A., Schreiber, G., and Fersht, A. (1994). Protein-protein recognition: crystal structural analysis of a barnase-barstar complex at 2.0-A resolution. Biochemistry, 33(30):8878-8889.

Burns, K. and Darwin, K. (2010). Pupylation : A Signal for Proteasomal Degradation in Mycobacterium tuberculosis. Subcell Biochem, 54:149-157.

Chaiet, L., Miller, T., Tausig, F., and Wolf, F. (1963). Antibiotic MSD-235. II. Separation and Purification of synergistic components. Antimicrob Agents Chemother (Bethesda), 161:28-32.

Clackson, T. and Lowman, H. (2004). Phage Display: A Practical Approach. Oxford University Press.

Crick, F. (1958). On Protein Synthesis. Symp. Soc. Exp. Biol., 12:138-163.

Crick, F. (1970). Central dogma of molecular biology. Nature, 227(5258):561-563.

Cuatrecasas, P., Wilchek, M., and Anfinsen, C. (1968). Selective enzyme purification by affinity chromatography. Proc Natl Acad Sci U S A, 61(2):636-643.

Davis, G., Elisee, C., Newham, D., and Harrison, R. (1999). New fusion protein systems designed to give soluble expression in Escherichia coli. Biotechnol Bioeng, 65(4):382-388.

Delphis, A., Beman, A., and L, B. (1719). Leeuwenhoek, A. van: Opera Omnia, seu Arcana Naturae ope exactissimorum Microscopiorum detecta, experimentis variis comprobata. Epistolis ad varios illustres viros $J$.

Dever, T. and Green, R. (2012). The elongation, termination, and recycling phases of translation in eukaryotes. Cold Spring Harb Perspect Biol, 4(7):a013706.

Deyev, S., Waibel, R., Lebedenko, E., Schubiger, A., and Plückthun, A. (2003). Design of multivalent complexes using the barnase*barstar module. Nat Biotechnol, 21(12):1486-1492.

di Guan, C., Li, P., Riggs, P., and Inouye, H. (1988). Vectors that facilitate the expression and purification of foreign peptides in Escherichia coli by fusion to maltose-binding protein. Gene, $67(1): 21-30$.

Doerfel, L. and Rodnina, M. (2013). Elongation factor P: Function and effects on bacterial fitness. Biopolymers, 99(11):837-845.

Doerfel, L., Wohlgemuth, I., Kothe, C., Peske, F., Urlaub, H., and Rodnina, M. (2013). EF$\mathrm{P}$ is essential for rapid synthesis of proteins containing consecutive proline residues. Science, 339(6115):85-88. 
Donnelly, N., Gorman, A., Gupta, S., and Samali, A. (2013). The eIF2alpha kinases: their structures and functions. Cell Mol Life Sci, 70(19):3493-3511.

Dougherty, W., Carrington, J., Cary, S., and Parks, T. (1988). Biochemical and mutational analysis of a plant virus polyprotein cleavage site. EMBO J, 7(5):1281-1287.

Duhamel, R., Schur, P., Brendel, K., and Meezan, E. (1979). pH gradient elution of human IgG1, IgG2 and IgG4 from protein A-sepharose. J Immunol Methods, 31(3-4):211-217.

Emsley, P. and Cowtan, K. (2004). Coot: model-building tools for molecular graphics. Acta Crystallogr D Biol Crystallogr, 60(Pt 12 Pt 1):2126-2132.

Engelsma, D., Valle, N., Fish, A., Salome, N., Almendral, J., and Fornerod, M. (2008). A supraphysiological nuclear export signal is required for parvovirus nuclear export. Mol Biol Cell, 19(6):2544-2552.

Enke, C. (2010). Towards a recombinant in vitro translation system from wheat. Cuvillier Verlag Göttingen.

Evan, G., Lewis, G., Ramsay, G., and Bishop, J. (1985). Isolation of monoclonal antibodies specific for human c-myc proto-oncogene product. Mol Cell Biol, 5(12):3610-3616.

Ezure, T., Suzuki, T., Higashide, S., Shintani, E., Endo, K., Kobayashi, S., Shikata, M., Ito, M., Tanimizu, K., and Nishimura, O. (2006). Cell-free protein synthesis system prepared from insect cells by freeze-thawing. Biotechnol Prog, 22(6):1570-1577.

Fornerod, M., Ohno, M., Yoshida, M., and Mattaj, I. (1997). CRM1 is an export receptor for leucine-rich nuclear export signals. Cell, 90(6):1051-1060.

Fraser, C., Lee, J., Mayeur, G., Bushell, M., Doudna, J., and Hershey, J. (2004). The j-subunit of human translation initiation factor eIF3 is required for the stable binding of eIF3 and its subcomplexes to $40 \mathrm{~S}$ ribosomal subunits in vitro. J Biol Chem, 279(10):8946-8956.

Frey, S. and Görlich, D. (2014a). A new set of highly efficient, tag-cleaving proteases for purifying recombinant proteins. J Chromatogr A, 1337:95-105.

Frey, S. and Görlich, D. (2014b). Purification of protein complexes of defined subunit stoichiometry using a set of orthogonal, tag-cleaving proteases. J Chromatogr A, 1337:106-115.

Frolova, L., Le Goff, X., Zhouravleva, G., Davydova, E., Philippe, M., and Kisselev, L. (1996). Eukaryotic polypeptide chain release factor eRF3 is an eRF1- and ribosome-dependent guanosine triphosphatase. RNA, 2(4):334-341.

Gallie, D. and Browning, K. (2001). eIF4G functionally differs from eIFiso4G in promoting internal initiation, cap-independent translation, and translation of structured mRNAs. J Biol Chem, 276(40):36951-36960.

Gaspar, N., Kinzy, T., Scherer, B., Humbelin, M., Hershey, J., and Merrick, W. (1994). Translation initiation factor eIF-2. Cloning and expression of the human cDNA encoding the gamma-subunit. J Biol Chem, 269(5):3415-3422.

Gibson, D., Young, L., Chuang, R., Venter, J., Hutchison, C. r., and Smith, H. (2009). Enzymatic assembly of DNA molecules up to several hundred kilobases. Nat Methods, 6(5):343-345. 
Goidl, J., Canaani, D., Boublik, M., Weissbach, H., and Dickerman, H. (1975). Polyanion-induced release of polyribosomes from HeLa cell nuclei. J Biol Chem, 250(23):9198-9205.

Görlich, D., Kostka, S., Kraft, R., Dingwall, C., Laskey, R., Hartmann, E., and Prehn, S. (1995). Two different subunits of importin cooperate to recognize nuclear localization signals and bind them to the nuclear envelope. Curr Biol, 5(4):383-392.

Görlich, D. and Kutay, U. (1999). Transport between the cell nucleus and the cytoplasm. Annu Rev Cell Dev Biol, 15:607-660.

Görlich, D., Pante, N., Kutay, U., Aebi, U., and Bischoff, F. (1996). Identification of different roles for RanGDP and RanGTP in nuclear protein import. EMBO J, 15(20):5584-5594.

Görlich, D., Prehn, S., Laskey, R., and Hartmann, E. (1994). Isolation of a protein that is essential for the first step of nuclear protein import. Cell, 79(5):767-778.

Green, N. (1990). Avidin and streptavidin. Methods Enzymol, 184:51-67.

Guillet, V., Lapthorn, A., Hartley, R., and Mauguen, Y. (1993). Recognition between a bacterial ribonuclease, barnase, and its natural inhibitor, barstar. Structure, 1(3):165-176.

Gupta, R. (1990). Sequence and structural homology between a mouse T-complex protein TCP-1 and the 'chaperonin' family of bacterial (GroEL, 60-65 kDa heat shock antigen) and eukaryotic proteins. Biochem Int, 20(4):833-841.

Gutierrez, E., Shin, B., Woolstenhulme, C., Kim, J., Saini, P., Buskirk, A., and Dever, T. (2013). eIF5A promotes translation of polyproline motifs. Mol Cell, 51(1):35-45.

Güttler, T., Madl, T., Neumann, P., Deichsel, D., Corsini, L., Mönecke, T., Ficner, R., Sattler, M., and Görlich, D. (2010). NES consensus redefined by structures of PKI-type and Rev-type nuclear export signals bound to CRM1. Nat Struct Mol Biol, 17(11):1367-1376.

Hartley, R. (1989). Barnase and barstar: two small proteins to fold and fit together. Trends Biochem Sci, 14(11):450-454.

Hartley, R. (1993). Directed mutagenesis and barnase-barstar recognition. Biochemistry, 32(23):5978-5984.

Hochuli, E., Döbeli, H., and Schacher, A. (1987). New metal chelate adsorbent selective for proteins and peptide containing neighbouring histidine residues. J Chromatogr.

Hopp, T., Pricket, K., Price, V., Libby, R., March, C., Ceretti, D., Urdal, D., and Conlon, P. (1988). A short polypeptide marker sequence useful for recombinant protein identification and purification. Bio/Technology.

Iborra, F., Jackson, D., and Cook, P. (2001). Coupled transcription and translation within nuclei of mammalian cells. Science, 293(5532):1139-1142.

Jackson, R., Hellen, C., and Pestova, T. (2010). The mechanism of eukaryotic translation initiation and principles of its regulation. Nat Rev Mol Cell Biol, 11(2):113-127.

Jackson, R. and Hunt, T. (1983). Preparation and use of nuclease-treated rabbit reticulocyte lysates for the translation of eukaryotic messenger RNA. Methods Enzymol, 96:50-74. 
Janknecht, R., de Martynoff, G., Lou, J., Hipskind, R., Nordheim, A., and HG, S. (1991). Rapid and efficient purification of native histidine-tagged protein expressed by recombinant vaccina virus. Proc Natl Acad Sci USA.

Janssen, G. and Moller, W. (1988). Kinetic studies on the role of elongation factors 1 beta and 1 gamma in protein synthesis. J Biol Chem, 263(4):1773-1778.

Jivotovskaya, A., Valasek, L., Hinnebusch, A., and Nielsen, K. (2006). Eukaryotic translation initiation factor 3 (eIF3) and eIF2 can promote mRNA binding to 40S subunits independently of eIF4G in yeast. Mol Cell Biol, 26(4):1355-1372.

Kabsch, W. (1993). Automatic processing of rotation diffraction data from crystals of initially unknown symmetry and cell constants. J Appl Crystallogr.

Kapp, L. and Lorsch, J. (2004). GTP-dependent recognition of the methionine moiety on initiator tRNA by translation factor eIF2. J Mol Biol, 335(4):923-936.

Karpeisky, M., Senchenko, V., Dianova, M., and Kanevsky, V. (1994). Formation and properties of S-protein complex with S-peptide-containing fusion protein. FEBS Lett, 339(3):209-212.

Kigawa, T., Yabuki, T., Matsuda, N., Matsuda, T., Nakajima, R., Tanaka, A., and Yokoyama, S. (2004). Preparation of Escherichia coli cell extract for highly productive cell-free protein expression. J Struct Funct Genomics, 5(1-2):63-68.

Kimball, S., Fabian, J., Pavitt, G., Hinnebusch, A., and Jefferson, L. (1998). Regulation of guanine nucleotide exchange through phosphorylation of eukaryotic initiation factor eIF2alpha. Role of the alpha- and delta-subunits of eiF2b. J Biol Chem, 273(21):12841-12845.

Kolade, O., Carr, S., Kuhlmann, U., Pommer, A., Kleanthous, C., Bouchcinsky, C., and Hemmings, A. (2002). Structural aspects of the inhibition of DNase and rRNase colicins by their immunity proteins. Biochimie, 84(5-6):439-446.

Korneeva, N., Lamphear, B., Hennigan, F., and Rhoads, R. (2000). Mutually cooperative binding of eukaryotic translation initiation factor (eIF) 3 and eIF4A to human eIF4G-1. J Biol Chem, 275(52):41369-41376.

Kovtun, O., Mureev, S., Jung, W., Kubala, M., Johnston, W., and Alexandrov, K. (2011). Leishmania cell-free protein expression system. Methods, 55(1):58-64.

Kozak, M. (1991). Structural features in eukaryotic mRNAs that modulate the initiation of translation. J Biol Chem, 266(30):19867-19870.

Kozak, M. (1994). Features in the 5' non-coding sequences of rabbit alpha and beta-globin mRNAs that affect translational efficiency. J Mol Biol, 235(1):95-110.

Kudo, N., Wolff, B., Sekimoto, T., Schreiner, E., Yoneda, Y., Yanagida, M., Horinouchi, S., and Yoshida, M. (1998). Leptomycin B inhibition of signal-mediated nuclear export by direct binding to CRM1. Exp Cell Res, 242(2):540-547.

Kutay, U., Izaurralde, E., Bischoff, F., Mattaj, I., and Görlich, D. (1997). Dominant-negative mutants of importin-beta block multiple pathways of import and export through the nuclear pore complex. EMBO J, 16(6):1153-1163.

Laemmli, U. (1970). Cleavage of structural proteins during the assembly of the head of bacteriophage T4. Nature, 227(5259):680-685. 
LeFebvre, A., Korneeva, N., Trutschl, M., Cvek, U., Duzan, R., Bradley, C., Hershey, J., and Rhoads, R. (2006). Translation initiation factor eIF4G-1 binds to eIF3 through the eIF3e subunit. J Biol Chem, 281(32):22917-22932.

Lejbkowicz, F., Goyer, C., Darveau, A., Neron, S., Lemieux, R., and Sonenberg, N. (1992). A fraction of the mRNA 5' cap-binding protein, eukaryotic initiation factor $4 \mathrm{E}$, localizes to the nucleus. Proc Natl Acad Sci U S A, 89(20):9612-9616.

Lindborg, M., Dubnovitsky, A., Olesen, K., Bjorkman, T., Abrahmsen, L., Feldwisch, J., and Hard, T. (2013). High-affinity binding to staphylococcal protein A by an engineered dimeric Affibody molecule. Protein Eng Des Sel, 26(10):635-644.

Lipowsky, G., Bischoff, F., Schwarzmaier, P., Kraft, R., Kostka, S., Hartmann, E., Kutay, U., and Görlich, D. (2000). Exportin 4: a mediator of a novel nuclear export pathway in higher eukaryotes. EMBO J, 19(16):4362-4371.

Lund, E. and Dahlberg, J. (1998). Proofreading and aminoacylation of tRNAs before export from the nucleus. Science, 282(5396):2082-2085.

Maag, D., Fekete, C., Gryczynski, Z., and Lorsch, J. (2005). A conformational change in the eukaryotic translation preinitiation complex and release of eIF1 signal recognition of the start codon. Mol Cell, 17(2):265-275.

Madin, K., Sawasaki, T., Ogasawara, T., and Endo, Y. (2000). A highly efficient and robust cellfree protein synthesis system prepared from wheat embryos: plants apparently contain a suicide system directed at ribosomes. Proc Natl Acad Sci U S A, 97(2):559-564.

Marblestone, J., Edavettal, S., Lim, Y., Lim, P., Zuo, X., and Butt, T. (2006). Comparison of SUMO fusion technology with traditional gene fusion systems: enhanced expression and solubility with SUMO. Protein Sci, 15(1):182-189.

Mariani, C., Gossele, V., de Beuckeller, M., de Block, M., Goldberg, R., de Greef, W., and Leemans, J. (1992). A chimaeric ribonuclease-inhibitor gene restores fertility to male sterile plants. Nature.

Marintchev, A. and Wagner, G. (2004). Translation initiation: structures, mechanisms and evolution. Q Rev Biophys, 37(3-4):197-284.

Masutani, M., Sonenberg, N., Yokoyama, S., and Imataka, H. (2007). Reconstitution reveals the functional core of mammalian eIF3. EMBO J, 26(14):3373-3383.

Méthot, N., Song, M., and Sonenberg, N. (1996). A region rich in aspartic acid, arginine, tyrosine, and glycine (DRYG) mediates eukaryotic initiation factor 4B (eIF4B) self-association and interaction with eIF3. Mol Cell Biol, 16(10):5328-5334.

Mikami, S., Kobayashi, T., Masutani, M., Yokoyama, S., and Imataka, H. (2008). A human cell-derived in vitro coupled transcription/translation system optimized for production of recombinant proteins. Protein Expr Purif, 62(2):190-198.

Mingot, J., Kostka, S., Kraft, R., Hartmann, E., and Görlich, D. (2001). Importin 13: a novel mediator of nuclear import and export. EMBO J, 20(14):3685-3694.

Mohr, D., Frey, S., Fischer, T., Guttler, T., and Gorlich, D. (2009). Characterisation of the passive permeability barrier of nuclear pore complexes. EMBO J, 28(17):2541-2553. 
Moks, T., Abrahmsen, L., Nilsson, B., Hellman, U., Sjoquist, J., and Uhlen, M. (1986). Staphylococcal protein A consists of five IgG-binding domains. Eur J Biochem, 156(3):637-643.

Mönecke, T., Güttler, T., Neumann, P., Dickmanns, A., Görlich, D., and Ficner, R. (2009). Crystal structure of the nuclear export receptor CRM1 in complex with Snurportin1 and RanGTP. Science, 324(5930):1087-1091.

Mossakowska, D., Nyberg, K., and Fersht, A. (1989). Kinetic characterization of the recombinant ribonuclease from Bacillus amyloliquefaciens (barnase) and investigation of key residues in catalysis by site-directed mutagenesis. Biochemistry, 28(9):3843-3850.

Mullis, K., Faloona, F., Scharf, S., Saiki, R., Horn, G., and Erlich, H. (1986). Specific enzymatic amplification of DNA in vitro: the polymerase chain reaction. Cold Spring Harb Symp Quant Biol, 51 Pt 1:263-273.

Nathanson, L., Xia, T., and Deutscher, M. (2003). Nuclear protein synthesis: a re-evaluation. $R N A, 9(1): 9-13$.

Nazina, T., Tourova, T., Poltaraus, A., Novikova, E., Grigoryan, A., Ivanova, A., Lysenko, A., Petrunyaka, V., Osipov, G., Belyaev, S., and Ivanov, M. (2001). Taxonomic study of aerobic thermophilic bacilli: descriptions of Geobacillus subterraneus gen. nov., sp. nov. and Geobacillus uzenensis sp. nov. from petroleum reservoirs and transfer of Bacillus stearothermophilus, Bacillus thermocatenulatus, Bacillus thermoleovorans, Bacillus kaustophilus, Bacillus thermodenitrificans to Geobacillus as the new combinations G. stearothermophilus, G. th. Int J Syst Evol Microbiol, 51(Pt 2):433-446.

Nielsen, K., Valasek, L., Sykes, C., Jivotovskaya, A., and Hinnebusch, A. (2006). Interaction of the RNP1 motif in PRT1 with HCR1 promotes 40S binding of eukaryotic initiation factor 3 in yeast. Mol Cell Biol, 26(8):2984-2998.

Niesen, F., Berglund, H., and Vedadi, M. (2007). The use of differential scanning fluorimetry to detect ligand interactions that promote protein stability. Nat Protoc, 2(9):2212-2221.

Nilsson, B., Moks, T., Jansson, B., Abrahmsen, L., Elmblad, A., Holmgren, E., Henrichson, C., Jones, T., and Uhlen, M. (1987). A synthetic IgG-binding domain based on staphylococcal protein A. Protein Eng, 1(2):107-113.

Nirenberg, M. and Matthaei, J. (1961). The dependence of cell-free protein synthesis in E. coli upon naturally occurring or synthetic polyribonucleotides. Proc Natl Acad Sci U S A, 47:1588-1602.

Nishi, K., Yoshida, M., Fujiwara, D., Nishikawa, M., Horinouchi, S., and Beppu, T. (1994). Leptomycin B targets a regulatory cascade of crm1, a fission yeast nuclear protein, involved in control of higher order chromosome structure and gene expression. J Biol Chem, 269(9):6320-6324.

Ohgushi, M. and Wada, A. (1983). 'Molten-globule state': a compact form of globular proteins with mobile side-chains. FEBS Lett, 164(1):21-24.

Olsen, D., Savner, E., Mathew, A., Zhang, F., Krishnamoorthy, T., Phan, L., and Hinnebusch, A. (2003). Domains of eIF1A that mediate binding to eIF2, eIF3 and eIF5B and promote ternary complex recruitment in vivo. EMBO J, 22(2):193-204.

Panavas, T., Sanders, C., and Butt, T. (2009). SUMO fusion technology for enhanced protein production in prokaryotic and eukaryotic expression systems. Methods Mol Biol, 497:303-317. 
Pantoliano, M., Petrella, E., Kwasnoski, J., Lobanov, V., Myslik, J., Graf, E., Carver, T., Asel, E., Springer, B., Lane, P., and Salemme, F. (2001). High-density miniaturized thermal shift assays as a general strategy for drug discovery. J Biomol Screen, 6(6):429-440.

Paraskeva, E., Izaurralde, E., Bischoff, F., Huber, J., Kutay, U., Hartmann, E., Lührmann, R., and Görlich, D. (1999). CRM1-mediated recycling of snurportin 1 to the cytoplasm. J Cell Biol, $145(2): 255-264$.

Park, H., Himmelbach, A., Browning, K., Hohn, T., and Ryabova, L. (2001). A plant viral rreinitiationffactor interacts with the host translational machinery. Cell, 106(6):723-733.

Passmore, L., Schmeing, T., Maag, D., Applefield, D., Acker, M., Algire, M., Lorsch, J., and Ramakrishnan, V. (2007). The eukaryotic translation initiation factors eIF1 and eIF1A induce an open conformation of the 40S ribosome. Mol Cell, 26(1):41-50.

Pelham, H. and Jackson, R. (1976). An efficient mRNA-dependent translation system from reticulocyte lysates. Eur J Biochem, 67(1):247-256.

Peng, L., Calton, G., and Burnett, J. (1987). Effect of borohydride reduction on antibodies. Appl Biochem Biotechnol, 14(2):91-99.

Pestova, T., Lomakin, I., Lee, J., Choi, S., Dever, T., and Hellen, C. (2000). The joining of ribosomal subunits in eukaryotes requires eIF5B. Nature, 403(6767):332-335.

Pestova, T., Shatsky, I., and Hellen, C. (1996). Functional dissection of eukaryotic initiation factor $4 \mathrm{~F}$ : the $4 \mathrm{~A}$ subunit and the central domain of the $4 \mathrm{G}$ subunit are sufficient to mediate internal entry of 43S preinitiation complexes. Mol Cell Biol, 16(12):6870-6878.

Phan, L., Schoenfeld, L., Valasek, L., Nielsen, K., and Hinnebusch, A. (2001). A subcomplex of three eIF3 subunits binds eIF1 and eIF5 and stimulates ribosome binding of mRNA and tRNA(i)Met. EMBO J, 20(11):2954-2965.

Phan, L., Zhang, X., Asano, K., Anderson, J., Vornlocher, H., Greenberg, J., Qin, J., and Hinnebusch, A. (1998). Identification of a translation initiation factor 3 (eIF3) core complex, conserved in yeast and mammals, that interacts with eIF5. Mol Cell Biol, 18(8):4935-4946.

Pisarev, A., Hellen, C., and Pestova, T. (2007). Recycling of eukaryotic posttermination ribosomal complexes. Cell, 131(2):286-299.

Pisarev, A., Skabkin, M., Pisareva, V., Skabkina, O., Rakotondrafara, A., Hentze, M., Hellen, C., and Pestova, T. (2010). The role of ABCE1 in eukaryotic posttermination ribosomal recycling. Mol Cell, 37(2):196-210.

Porath, J., Carlsson, J., Olsson, I., and Belfrage, G. (1975). Metal chelate affinity chromatography, a new approach to protein fractionation. Nature, 258(5536):598-599.

Pryor, K. and Leiting, B. (1997). High-level expression of soluble protein in Escherichia coli using a His6-tag and maltose-binding-protein double-affinity fusion system. Protein Expr Purif, 10(3):309-319.

Querol-Audi, J., Sun, C., Vogan, J., Smith, M., Gu, Y., Cate, J., and Nogales, E. (2013). Architecture of human translation initiation factor 3. Structure, 21(6):920-928. 
Rabl, J., Leibundgut, M., Ataide, S., Haag, A., and Ban, N. (2011). Crystal structure of the eukaryotic 40S ribosomal subunit in complex with initiation factor 1. Science, 331(6018):730736 .

Reichelt, R., Holzenburg, A., Buhle, E. J., Jarnik, M., Engel, A., and Aebi, U. (1990). Correlation between structure and mass distribution of the nuclear pore complex and of distinct pore complex components. J Cell Biol, 110(4):883-894.

Ribbeck, K. and Görlich, D. (2001). Kinetic analysis of translocation through nuclear pore complexes. EMBO J, 20(6):1320-1330.

Ribbeck, K., Lipowsky, G., Kent, H., Stewart, M., and Görlich, D. (1998). NTF2 mediates nuclear import of Ran. EMBO J, 17(22):6587-6598.

Roberts, B. and Paterson, B. (1973). Efficient translation of tobacco mosaic virus RNA and rabbit globin 9S RNA in a cell-free system from commercial wheat germ. Proc Natl Acad Sci USA, $70(8): 2330-2334$.

Rogers, G. J., Richter, N., Lima, W., and Merrick, W. (2001). Modulation of the helicase activity of eIF4A by eIF4B, eIF4H, and eIF4F. J Biol Chem, 276(33):30914-30922.

Russell, D. and Spremulli, L. (1979). Purification and characterization of a ribosome dissociation factor (eukaryotic initiation factor 6) from wheat germ. J Biol Chem, 254(18):8796-8800.

Ruud, K., Kuhlow, C., Goss, D., and Browning, K. (1998). Identification and characterization of a novel cap-binding protein from Arabidopsis thaliana. J Biol Chem, 273(17):10325-10330.

Sachdev, D. and Chirgwin, J. (1999). Properties of soluble fusions between mammalian aspartic proteinases and bacterial maltose-binding protein. J Protein Chem, 18(1):127-136.

Safarik, I. and Safarikova, M. (2004). Magnetic techniques for the isolation and purification of proteins and peptides. Biomagn Res Technol, 2(1):7.

Saini, A., Nanda, J., Martin-Marcos, P., Dong, J., Zhang, F., Bhardwaj, M., Lorsch, J., and Hinnebusch, A. (2014). Eukaryotic translation initiation factor eIF5 promotes the accuracy of start codon recognition by regulating $\mathrm{Pi}$ release and conformational transitions of the preinitiation complex. Nucleic Acids Res.

Sambrook, J. and Russell, D. (2001). Molecular Cloning: A Laboratory Manual. Cold Spring Harbor Laboratory Press.

Sano, T., Pandori, M., Chen, X., Smith, C., and Cantor, C. (1995). Recombinant core streptavidins. A minimum-sized core streptavidin has enhanced structural stability and higher accessibility to biotinylated macromolecules. J Biol Chem, 270(47):28204-28209.

Schatz, P. (1993). Use of peptide libraries to map the substrate specificity of a peptide-modifying enzyme: a 13 residue consensus peptide specifies biotinylation in Escherichia coli. Biotechnology (N Y), 11(10):1138-1143.

Schmidt, T., Koepke, J., Frank, R., and Skerra, A. (1996). Molecular interaction between the Strep-tag affinity peptide and its cognate target, streptavidin. J Mol Biol, 255(5):753-766.

Schreiber, G. (2001). Methods for studying the interaction of barnase with its inhibitor barstar. Methods Mol Biol, 160:213-226. 
Schreiber, G. and Fersht, A. (1993). Interaction of barnase with its polypeptide inhibitor barstar studied by protein engineering. Biochemistry, 32(19):5145-5150.

Shalev, A., Valasek, L., Pise-Masison, C., Radonovich, M., Phan, L., Clayton, J., He, H., Brady, J., Hinnebusch, A., and Asano, K. (2001). Saccharomyces cerevisiae protein Pci8p and human protein eIF3e/Int-6 interact with the eIF3 core complex by binding to cognate eIF3b subunits. $J$ Biol Chem, 276(37):34948-34957.

Shevchenko, A., Wilm, M., Vorm, O., Jensen, O., Podtelejnikov, A., Neubauer, G., Shevchenko, A., Mortensen, P., and Mann, M. (1996). A strategy for identifying gel-separated proteins in sequence databases by MS alone. Biochem Soc Trans, 24(3):893-896.

Shimizu, Y., Inoue, A., Tomari, Y., Suzuki, T., Yokogawa, T., Nishikawa, K., and Ueda, T. (2001). Cell-free translation reconstituted with purified components. Nat Biotechnol, 19(8):751-755.

Siridechadilok, B., Fraser, C., Hall, R., Doudna, J., and Nogales, E. (2005). Structural roles for human translation factor eIF3 in initiation of protein synthesis. Science, 310(5753):1513-1515.

Smith, D. and Johnson, K. (1988). Single-step purification of polypeptides expressed in Escherichia coli as fusions with glutathione S-transferase. Gene, 67(1):31-40.

Smyth, D., Blumenfeld, O., and Königsberg, W. (1964). Reactions of N-ethylmaleimide with peptides and amino acids. Biochem $J, 91(3): 589-595$.

Smyth, D., Mrozkiewicz, M., McGrath, W., Listwan, P., and Kobe, B. (2003). Crystal structures of fusion proteins with large-affinity tags. Protein Sci, 12(7):1313-1322.

Sørensen, H., Sperling-Petersen, H., and Mortensen, K. (2003). A favorable solubility partner for the recombinant expression of streptavidin. Protein Expr Purif, 32(2):252-259.

Staak, C., Salchow, F., Clausen, P., and Luge, E. (1996). Polystyrene as an affinity chromatography matrix for the purification of antibodies. J Immunol Methods, 194(2):141-146.

Starkenstein, E. (1910). Über Fermentwirkung und deren Beeinflussung durch Neutralsalze. Biochem. Z.

Stemp, M., Guha, S., Hartl, F., and Barral, J. (2005). Efficient production of native actin upon translation in a bacterial lysate supplemented with the eukaryotic chaperonin TRiC. Biol Chem, $386(8): 753-757$.

Stüven, T., Hartmann, E., and Görlich, D. (2003). Exportin 6: a novel nuclear export receptor that is specific for profilin.actin complexes. EMBO J, 22(21):5928-5940.

Sun, C., Todorovic, A., Querol-Audi, J., Bai, Y., Villa, N., Snyder, M., Ashchyan, J., Lewis, C., Hartland, A., Gradia, S., Fraser, C., Doudna, J., Nogales, E., and Cate, J. (2011). Functional reconstitution of human eukaryotic translation initiation factor 3 (eIF3). Proc Natl Acad Sci U $S$ A, 108(51):20473-20478.

Tausig, F. and Wolf, F. (1964). Streptavidin-a substance with avidin-like properties produced by microorganisms. Biochem Biophys Res Commun, 14:205-209.

Terry, L., Shows, E., and Wente, S. (2007). Crossing the nuclear envelope: hierarchical regulation of nucleocytoplasmic transport. Science, 318(5855):1412-1416. 
Udem, S. and Warner, J. (1973). The cytoplasmic maturation of a ribosomal precursor ribonucleic acid in yeast. $J$ Biol Chem, 248(4):1412-1416.

Valasek, L., Nielsen, K., and Hinnebusch, A. (2002). Direct eIF2-eIF3 contact in the multifactor complex is important for translation initiation in vivo. EMBO J, 21(21):5886-5898.

Voss, S. and Skerra, A. (1997). Mutagenesis of a flexible loop in streptavidin leads to high affinity for the Strep-tag II peptode ad improved performance in recombinant protein purification. Protein Eng.

Wen, W., Meinkoth, J., Tsien, R., and Taylor, S. (1995). Identification of a signal for rapid export of proteins from the nucleus. Cell, 82(3):463-473.

Williams, A., Hill, S., and Ibrahim, I. (1981). Improved spectrophotometric methods for the assay of carbodiimides. Anal Biochem, 114(1):173-176.

$\mathrm{Xi}, \mathrm{F}$. and $\mathrm{Wu}, \mathrm{J}$. (2004). Macroporous chitosan layer coated on non-porous silica gel as a support for metal chelate affinity chromatographic adsorbent. J Chromatogr A, 1057(1-2):41-47.

Zhou, M., Sandercock, A., Fraser, C., Ridlova, G., Stephens, E., Schenauer, M., Yokoi-Fong, T., Barsky, D., Leary, J., Hershey, J., Doudna, J., and Robinson, C. (2008). Mass spectrometry reveals modularity and a complete subunit interaction map of the eukaryotic translation factor eIF3. Proc Natl Acad Sci U S A, 105(47):18139-18144. 


\section{Abbreviations}

\begin{tabular}{|c|c|}
\hline aa & Amino acid \\
\hline ASP & Ammonium Sulfate Precipitation \\
\hline ATP & Adenosine triphosphate \\
\hline bp & Base pairs \\
\hline BSA & Bovine serum albumin \\
\hline C-terminal & Carboxy-terminal \\
\hline DMSO & Dimethylsulfoxide \\
\hline DNA & Deoxyribonucleic acid \\
\hline DTT & Dithiothreitol \\
\hline dTTP & Deoxythymidine triphosphate \\
\hline EDTA & Ethylenediaminetetraacetic acid \\
\hline $\mathrm{eIF}$ & eukaryotic translation initiation factor \\
\hline FT & Flow through \\
\hline GFP & Green fluorescent protein \\
\hline GTP (GDP) & Guanosine triphosphate (guanosine diphosphate) \\
\hline hr & $\operatorname{Hour}(\mathrm{s})$ \\
\hline $\operatorname{IgG}$ & Immunoglobin $\mathrm{G}$ \\
\hline IP & Immunoprecipitation \\
\hline $\mathrm{m} 7 \mathrm{G}$ & 7-methylguanosine cap \\
\hline Met-tRNA $_{i}{ }^{\text {Met }}$ & Aminoacylated initiator tRNA \\
\hline $\mathrm{MgAc}$ & Magnesium acetate \\
\hline $\min$ & Minute(s) \\
\hline mRNA & Messenger ribonucleic acid \\
\hline MS & Mass Spectrometry \\
\hline NE & Nuclear envelope \\
\hline Ni-NTA & Nickel-nitrilo triacetic acid \\
\hline NPC & Nuclear pore complex \\
\hline N-terminal & Amino-terminal \\
\hline NTR & Nuclear transport receptor \\
\hline OD & Optical density \\
\hline $\mathrm{o} / \mathrm{n}$ & over night \\
\hline ORF & Open reading frame \\
\hline PBS & Phosphate-buffered saline \\
\hline PCR & Polymerase chain reaction \\
\hline PIC & Preinitiation Complex \\
\hline PMSF & Phenylmethanesulfonylfluoride \\
\hline $\mathrm{RF}$ & Release factor \\
\hline RNA & Ribonucleic acid \\
\hline rmp & Rounds per minute \\
\hline RRL & Rabbit Reticulocyte Lysate \\
\hline
\end{tabular}


RT

SDS-PAGE

sec

TC

TCEP

TEV

tRNA

Tris

$\mathrm{v} / \mathrm{v}$

WGE

$\mathrm{w} / \mathrm{v}$

ZZ-
Room temperature

Sodium dodecyl sulfate polyacrylamide gel electrophoresis Second(s)

Ternary complex

Tris(2-carboxyethyl)phosphine

Tobacco etch virus

Transfer RNA

Tris(hydroxymethyl)aminomethane

volume/volume

Wheat Germ Extract

weight/volume

IgG-binding domain of Staphylococcus proteinA (in tandem) 


\section{Acknowledgements - Danksagung}

During the last four years I had the wonderful privilege to constantly have people around me that were supportive, sincere and caring. It does sound like a cliché, but I honestly would not have made it without you.

First I want to thank my supervisor Prof. Dirk Görlich. During the last four years you taught me what being a scientist is all about. How to ask the right questions and how to address them by elegant and straight forward experiments. I am thankful for all the experiences I was allowed to make.

Further I want to thank my additional Thesis Committee members Prof. Marina Rodnina and Prof. Volker Lipka. Although I confronted you with a multitude of different ideas and approaches every time we met, you were always interested and supportive with good comments and ideas on hand.

I would also like to thank Prof. Doenecke, Prof. Urlaub and Prof. Wintermeyer for accepting to be part of my extended Thesis Committee.

Further I would like to thank the IMPRS Molecular Biology, specially Dr. Steffen Burkhardt and Kerstin Grüniger for supporting me throughout the last five years.

A huge "Thank You" goes to our wonderful technicians, with whom is was always a great pleasure to work. Jens, I thank you for your constant help and support during wheat germ extract production and in front of that blackbox called Susi. Both things would have been far less fun without you! Susanne and Gabi, thank you so much for the enormous amount of plasmids you cloned and purified for me. Jürgen, I am extremely grateful for all your help and support mainly during the last few months. It allowed me to focus on writing without all the other things pausing. Heike and Renate, thank you for supplying me with plasmids and lysates and always offering help and support. Knowing that I can always count on all of you is priceless. Further I would like to acknowledge my awesome lab rotation students Metin Aksu and Muna Khan.

Cathrin, you supported me during my lab rotation and the first year of Ph.D. You shared all your experience and wisdom with me, showed me how the 10600 department works and carefully introduced me into the depths of the project. I never had the feeling we were competing but working side by side to get the project to work. I am sorry it did not work out the way both of us wished it would.

Steffen, I not only want to thank you for supplying me with plasmids and proteins from your seemingly infinite treasure chamber (also called freezer), but also for the scientific and non-scientific discussions we had. Many of these conversations were not only informative but also extremely encouraging and supportive.

My dear colleagues, I want to thank all of you for providing a really nice working atmosphere. I am grateful for all the scientific chats (special thanks to Koray, Kevser, Hema, 
Broder, Bastian, Metin, Tino, Volker and Micha), for sharing plasmids, proteins and chemicals with me and also for the one or the other barbecue, breakfast or football match.

Den Rest gibt es nun auf Deutsch:

Ich möchte mich ganz herzlich bei meiner Kaffee - Runde bedanken. Jens, Micha, Broder, Philip, Bernard und Renate, ihr seid die Besten. Ich will garnicht wissen wie oft ich meinen Frust bei euch abgeladen habe, wie oft ihr mich zum lachen gebracht habt, wie oft ihr mich in den Arm nehmen musstet oder mir Taschentücher zugesteckt habt. Euch jeden Tag um mich zu haben ist ein riesen Geschenk und dafür danke ich euch aus tiefstem Herzen!

Mein Labor 5 darf natürlich auch nicht fehlen: Heike, Susanne, Cathrin, Tino, Micha und seit Neustem auch Anne. Mit euch ist und war es immer lustig. Was kleine Neckereien, Musik und der ein oder andere Plausch doch positives bewirken kann. Ich fühl mich bei euch pudelwohl und vor allem der Anfangscrew danke ich, das ihr mich Küken so lieb und mit offenen Armen aufgenommen habt.

Ein Danke geht auch an Heinz-Jürgen, ohne dein Wandern wäre Deutschland nicht Weltmeister geworden, davon bin ich überzeugt :). Auch Conny möchte ich von ganzem Herzen danken, dafür das du uns Wissenschaftler zu Ausflügen und Wanderungen überredest und natürlich für unsere legendäre Weihnachtsbäckerei!

Natürlich braucht es auch Leute außerhalb des Labors. Cadu, David und Simone, ich danke euch für zwei wunderschöne Horizons - Jahre, die gespickt waren mit dem ein oder anderen Konzert oder Fussball Besuch und natürlich viel fantastischem Essen. Tine, ohne dich wäre die Zeit in Göttingen sehr viel weniger chaotisch, bunt und verrückt gewesen. Mir fallen auf Anhieb ein Dutzend Geschichten ein und sofort muss ich grinsen - und ich mag keine davon missen. Nicht fehlen dürfen auch Lisette und klein Neo, Thomas, Tascha, Claudia und meine liebe Kathrin.

Zum Schluß möchte ich mich bei Maya, Christoph und meinen wundervollen Eltern bedanken. Ihr schafft es mich mit eurer ehrlichen, geduldigen und liebevollen Art und Weise jeden Tag in dem zu bestärken was ich tue. Zu wissen, das ihr immer für mich da seid auch wenn es mal schwierig ist - ist unbeschreiblich schön. 


\title{
Curriculum Vitae
}

\author{
Jennifer C. Seefeldt \\ born on June, $3^{\text {rd }} 1987$ in Ellwangen, Germany \\ Max Planck Institute for Biophysical Chemistry \\ Department of Cellular Logistics \\ Am Fassberg 11, 37077 Göttingen, Germany \\ Phone: +49 (0) 5512012454 \\ E-Mail: Jennifer.Seefeldt@mpibpc.mpg.de
}

EdUCATION AND RESEARCh EXPERIENCE

2011 - present Max Planck Institute for Biophysical Chemistry, Göttingen, Germany: Doctoral Thesis: "Analyzing the eukaryotic translation initiation apparatus and new approaches in affinity chromatography"

2011 Max Planck Institute for Biophysical Chemistry, Göttingen, Germany: Master Thesis: "Towards the reconstitution of recombinant eukaryotic translation initiation factor 3 (eIF3) from Triticum aestivum"

2010 Georg August University, Göttingen, Germany:

Internship in the Department of Molecular Structural Biology

2010

Max Planck Institute for Biophysical Chemistry, Göttingen, Germany: Internship in the Department of Chromatin Biochemistry

2009 - present Georg August University, Göttingen, Germany and Max Planck International Research School, Göttingen, Germany: MSc/PhD Program Molecular Biology

2009

Albrecht von Haller Institute for Plant Sciences, Göttingen, Germany: Bachelor Thesis: "Optimizing the biosynthesis of wax esters by retargeting of biosynthetic enzymes"

2008 CombinatoRX, Singapore, Singapore:

Summer Internship, Virology

2006 - 2009 Georg August University, Göttingen, Germany:

Bachelor of Science Program Biology 
1998 - 2006 Carolus Magnis Gymnasium, Marsberg, Germany:

Abitur degree (general qualification for university entrance)

1993 - 1998 German European School, Singapore, Singapore

SCHOLARSHIPS

2010 - present Max Planck Institute "Promotionsstipendium"

2009 - $2010 \quad$ International Max Planck Research School support

Voluntary ACtivities

2012

Member of the organization board for the "Women Career and Network (WoCaNet)" Symposium in Göttingen

2010 - 2012 Main organizer of the annual "Horizons in Molecular Biology" PhD Symposium 